\title{
Nickel-Catalyzed Coupling of Alkenes, Aldehydes, and Silyl Triflates
}

\author{
Ng, S.-S.; Ho, C.-Y.; Jamison, T. F. * \\ Department of Chemistry, Massachusetts Institute of Technology, Cambridge, MA 02139
}

\section{Supporting Information}

Experimental Procedures, Analytical and Spectroscopic Data for Compounds $1 \mathbf{a}-\mathbf{5 b}$.

Pages S2 - S50

\author{
${ }^{1} \mathrm{H}$ and ${ }^{13} \mathrm{C}$ NMR spectra for compounds $\mathbf{1 a}-\mathbf{5 b}$. \\ Pages S51 - S164
}




\section{General Information.}

Unless otherwise noted, all reactions were performed under an oxygen-free atmosphere of nitrogen or argon with rigid exclusion of moisture from reagents and glassware. Tetrahydrofuran was distilled from a blue solution of sodium benzophenone ketyl. Dichloromethane and toluene was distilled from calcium hydride. Aromatic aldehydes were purchased from Aldrich Chemical Co. and used as received. Other aldehydes were distilled and saturated with nitrogen before use. Bis(cyclooctadienyl)nickel $(0) \quad\left(\mathrm{Ni}(\operatorname{cod})_{2}\right) \quad$ and tris-(o-methoxyphenyl)-phosphine and triphenylphosphine were purchased from Strem Chemicals, Inc., stored under nitrogen atmosphere and used without further purification. Ethylene was purchased from BOC Gases and used as received. 1-octene was purchased from Alfa Aesar and used as received. All other alkenes were purchased from Aldrich Chemical Co. and used as received. Dicyclohexylphenylphosphine and ethyldiphenylphosphinite were purchased from Aldrich Chemical Co., stored under nitrogen atmosphere and used without further purification. Triethylsilyltrifluoromethanesulfonate (TESOTf) and trimethylsilyltrifluoromethansulfonate (TMSOTf) were purchased from Aldrich Chemical Co. and were distilled over calcium hydride before use. Tert-butyldimethysilyltrifluoromethanesulfonate (TBSOTf) was purchased from Alfa Aesar and was distilled over calcium hydride before use.

Analytical thin layer chromatography (TLC) was performed using EM Science silica gel 60 $\mathrm{F}_{254}$ plates. The developed chromatogram was analyzed by UV lamp (254 nm), ethanolic phosphomolybdic acid (PMA) or potassium permanganate $\left(\mathrm{KMnO}_{4}\right)$. Liquid chromatography was performed using a forced flow (flash chromatography) of the indicated solvent system on Silicycle Silica Gel (230 - $400 \mathrm{mesh}) .{ }^{1} \mathrm{H}$ and ${ }^{13} \mathrm{C}$ NMR spectra were recorded on Varian 300 $\mathrm{MHz}$, Varian $500 \mathrm{MHz}$ or Bruker $400 \mathrm{MHz}$ spectrometers in $\mathrm{CDCl}_{3}$ or $\mathrm{C}_{6} \mathrm{D}_{6}$, unless otherwise noted. Chemical shifts in ${ }^{1} \mathrm{H}$ NMR spectra are reported in parts per million (ppm) on the $\delta$ scale from an internal standard of residual chloroform $(7.27 \mathrm{ppm})$ or residual benzene $(7.16 \mathrm{ppm})$. Data are reported as follows: chemical shift, multiplicity $(\mathrm{s}=$ singlet, $\mathrm{d}=$ doublet, $\mathrm{t}=$ triplet, $\mathrm{q}=$ quartet, $\mathrm{m}=$ multiplet, $\mathrm{b}=$ broad), coupling constant in hertz $(\mathrm{Hz})$, and integration. Chemical shifts of ${ }^{13} \mathrm{C}$ NMR spectra are reported in ppm from the central peak of $\mathrm{CDCl}_{3}$ (77.23 ppm) on the $\delta$ scale. Infrared (IR) spectra were recorded on a Perkin-Elmer 2000 FT-IR. High resolution mass spectra (HRMS) were obtained on a Bruker Daltonics APEXII 3 Tesla Fourier Transform Mass Spectrometer by Dr. Li Li of the Massachusetts Institute of Technology Department of Chemistry Instrument Facility. Chiral GC analysis was performed on a Varian CP-3800 gas chromatograph fitted with Chiraldex B-PH, B-DA, and G-TA capillary columns. Chiral HPLC analysis was performed on a Hewlett-Packard 1100 chromatograph equipped with a variable wavelength detector and Chiralcel OD or OD-H columns. 


\section{Preparation of 2,2-dimethyl-3-oxo-propionic acid methyl ester}<smiles>COC(=O)C(C)(C)C=O</smiles>

2,2-dimethyl-3-oxo-propionic acid methyl ester. 3-Hydroxy-2,2dimethyl-propionic acid methyl ester $(15 \mathrm{~g}, 113 \mathrm{mmol})$ in $200 \mathrm{~mL}$ dichloromethane was cooled to $0^{\circ} \mathrm{C}$. Pyridinium chlorochromate $(43 \mathrm{~g}, 200 \mathrm{mmol})$ was added. The mixture was slowly warmed to room temperature and stirred $24 \mathrm{~h}$. The crude in dichloromethane was filtered through silica gel. Celite was added to the remaining black viscous oil from the reaction mixture until the viscous oil is all absorbed to the celite. Dichloromethane was added to this slurry and the dichloromethane solution was filtered through silica gel. Dichloromethane was removed at reduced pressure ( 80 Torr) to give a pale yellow crude oil. Fractional distillation removed residue dichloromethane and obtained 2,2-dimethyl-3-oxo-propionic acid methyl ester as a colorless oil ( $7 \mathrm{~g}, 48 \%$ yield).

${ }^{1} \mathrm{H}$ NMR (300 MHz, $\left.\mathrm{CDCl}_{3}, \delta\right): 9.60$ (s, 1H); 3.70 (s, 3H); 1.29 (s, 6H).

${ }^{13} \mathrm{C}$ NMR $\left(75 \mathrm{MHz}, \mathrm{CDCl}_{3}, \delta\right): 199.1,173.2,53.9,52.6,19.7$.

IR (NaCl, thin film): 2988, 2958, 1726, 1468, 1278, 1151, 866.

\section{Nickel-catalyzed couplings of ethylene and aldehydes (1a, 1b, 1c, 1d, 1i, 1j, 1l, 1m, 1n).}

General procedure 1. A $10 \mathrm{~mL}$ round bottom flask and a stir bar were oven-dried and brought into a glove box. $\mathrm{Ni}(\operatorname{cod})_{2} \quad(27.5 \mathrm{mg}, \quad 0.1 \mathrm{mmol}, 20 \mathrm{~mol} \%)$ and tris- $o$-methoxyphenylphosphine $(70.5 \mathrm{mg}, 0.2 \mathrm{mmol}, 40 \mathrm{~mol} \%$ ) were added to the round bottom flask, the flask was sealed with a septum, and the sealed flask was brought out of the glove box and connected to an argon line. The catalyst mixture was dissolved in toluene $(2.5 \mathrm{~mL})$ under argon and stirred $15 \mathrm{~min}$ at room temperature. The reaction mixture was purged with ethylene for 1 min to remove argon, taken care not to introduce oxygen. The ethylene atmosphere was maintained with an ethylene balloon. Triethylamine $(418 \mu \mathrm{L}, 3 \mathrm{mmol}, 600 \mathrm{~mol} \%)$ was added. Aldehyde (0.5 mmol, $100 \mathrm{~mol} \%$, as specified) was added. Silyltriflate $(0.875 \mathrm{mmol}, 175 \mathrm{~mol} \%$, as specified) was added. The mixture was stirred at room temperature for $3-18 \mathrm{~h}$, as judged by the TLC of the reaction mixture. The mixture was filtered through a plug of silica gel. Solvent was removed under reduced pressure and the crude mixture was diluted in hexane. Purification via flash chromatography on silica afforded the coupling product. 


\section{Nickel-catalyzed couplings of ethylene and aldehydes (1e, 1f, $1 \mathrm{~g}, 1 \mathrm{~h}, 1 \mathrm{k})$.}

General procedure 2. A $10 \mathrm{~mL}$ round bottom flask and a stir bar were oven-dried and brought into a glove box. $\mathrm{Ni}(\operatorname{cod})_{2} \quad(27.5 \mathrm{mg}, \quad 0.1 \mathrm{mmol}, \quad 20 \mathrm{~mol} \%)$, tris- $o$-methoxyphenylphosphine $(70.5 \mathrm{mg}, 0.2 \mathrm{mmol}, 40 \mathrm{~mol} \%)$ and aldehyde $(0.5 \mathrm{mmol}, 100$ mol\%) were added to the round bottom flask, the flask was sealed with a septum, and the sealed flask was brought out of the glove box and connected to an argon line. The catalyst mixture was dissolved in toluene $(2.5 \mathrm{~mL})$ under argon and stirred $15 \mathrm{~min}$ at room temperature. The reaction mixture was purged with ethylene for $1 \mathrm{~min}$ to remove argon, taken care not to introduce oxygen. The ethylene atmosphere was maintained with an ethylene balloon. Next triethylamine $(418 \mu \mathrm{L}$, $3 \mathrm{mmol}, 600 \mathrm{~mol} \%)$ was added. Silyltriflate $(0.875 \mathrm{mmol}, 175 \mathrm{~mol} \%$, as specified) was added. The mixture was stirred at room temperature for $3-18 \mathrm{~h}$, as judged by TLC of the reaction mixture. The mixture was filtered through a plug of silica gel. Solvent was removed under reduced pressure and the crude mixture was diluted in hexane. Purification via flash chromatography on silica afforded the coupling product.

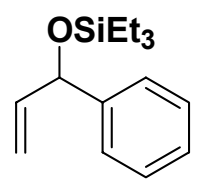

(1a)

The reaction of ethylene and benzaldehyde $(51 \mu \mathrm{L}, 0.5 \mathrm{mmol})$ with $\mathrm{Ni}(\operatorname{cod})_{2}$, tris- $O$-methoxyphenylphosphine and TESOTf $(197 \mu \mathrm{L}, 0.875 \mathrm{mmol})$, triethylamine in toluene following the general procedure 1 above, afforded $\mathbf{1 a}$ in $82 \%$ isolated yield as a colorless oil.

${ }^{1} \mathrm{H}$ NMR (400 MHz, $\left.\mathrm{CDCl}_{3}, \delta\right): 7.32-7.45(\mathrm{~m}, 4 \mathrm{H}) ; 7.29(\mathrm{t}, J=7.0 \mathrm{~Hz}, 1 \mathrm{H}) ; 6.01$ (ddd, $J=6.0$, $10.2,16.9 \mathrm{~Hz}, 1 \mathrm{H}) ; 5.34(\mathrm{dt}, J=1.5,16.9 \mathrm{~Hz}, 1 \mathrm{H}) ; 5.25(\mathrm{~d}, J=5.9 \mathrm{~Hz}, 1 \mathrm{H}) ; 5.13(\mathrm{dt}, J=1.5$, $10.2 \mathrm{~Hz}, 1 \mathrm{H}) ; 0.99$ (t, $J=8.0 \mathrm{~Hz}, 9 \mathrm{H}) ; 0.66(\mathrm{dq}, J=1.8,7.8 \mathrm{~Hz}, 6 \mathrm{H})$.

${ }^{13} \mathrm{C} \mathrm{NMR}\left(100 \mathrm{MHz}, \mathrm{CDCl}_{3}, \delta\right): 143.9,141.8,128.4,127.3,126.2,113.7,75.9,7.0,5.1$. IR (NaCl, thin film): 2956, 2877, 1640, 1454, 1240, 1065, 744, 699.

HRMS-ESI (m / z): [M + Na $]^{+}$calcd for $\mathrm{C}_{15} \mathrm{H}_{24} \mathrm{OSiNa}$, 271.149; found, 271.150. 


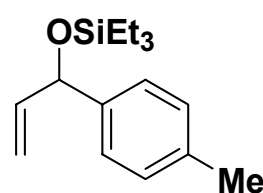

(1b)

The reaction of ethylene and $p$-tolualdehyde $(59 \mu \mathrm{L}, 0.5 \mathrm{mmol})$ with $\mathrm{Ni}(\operatorname{cod})_{2}$, tris-o-methoxyphenylphosphine and TESOTf $(197 \mu \mathrm{L}, 0.875 \mathrm{mmol})$, triethylamine in toluene following the general procedure 1 above, afforded $\mathbf{1 b}$ in $88 \%$ isolated yield as a colorless oil. ${ }^{1} \mathrm{H}$ NMR (400 MHz, $\mathrm{CDCl}_{3}, \delta$ ): $7.27(\mathrm{~d}, J=8.0,2 \mathrm{H}) ; 7.16(\mathrm{~d}, J=8.0 \mathrm{~Hz}, 2 \mathrm{H}) ; 5.97$ (ddd, $J=5.9$, $10.2,16.9 \mathrm{~Hz}, 1 \mathrm{H}) ; 5.30(\mathrm{dt}, J=1.5,17.0 \mathrm{~Hz}, 1 \mathrm{H}) ; 5.17(\mathrm{~d}, J=5.9 \mathrm{~Hz}, 1 \mathrm{H}) ; 5.09$ (dt, $J=1.3$, $10.2 \mathrm{~Hz}, 1 \mathrm{H}) ; 2.37$ (s, 3H); 0.97 (t, $J=7.9 \mathrm{~Hz}, 9 \mathrm{H}) ; 0.65$ (dq, $J=1.9,7.5 \mathrm{~Hz}, 6 \mathrm{H})$.

${ }^{13} \mathrm{C}$ NMR $\left(100 \mathrm{MHz}, \mathrm{CDCl}_{3}, \delta\right): 142.1,141.1,136.8,129.1,126.2,113.4,75.8,21.3,7.0,5.2$. IR (NaCl, thin film): 2955, 2877, 1640, 1513, 1458, 1415, 1079, 1007, 844. HRMS-ESI (m / z): [M + Na $]^{+}$calcd for $\mathrm{C}_{16} \mathrm{H}_{26} \mathrm{OSiNa}$, 285.165; found, 285.165.

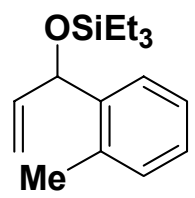

(1c)

The reaction of ethylene and $o$-tolualdehyde $(58 \mu \mathrm{L}, 0.5 \mathrm{mmol})$ with $\mathrm{Ni}(\mathrm{cod})_{2}$, tris-o-methoxyphenylphosphine and TESOTf $(197 \mu \mathrm{L}, 0.875 \mathrm{mmol})$, triethylamine in toluene following the general procedure 1 above, afforded $1 \mathbf{c}$ in $93 \%$ isolated yield as a colorless oil.

${ }^{1} \mathrm{H}$ NMR $\left(400 \mathrm{MHz}, \mathrm{CDCl}_{3}, \delta\right): 7.50(\mathrm{~d}, J=7.0,1 \mathrm{H}) ; 7.11-7.24(\mathrm{~m}, 3 \mathrm{H}) ; 5.93$ (ddd, $J=5.7$, $10.2,17.0 \mathrm{~Hz}, 1 \mathrm{H}) ; 5.36(\mathrm{~d}, J=5.6 \mathrm{~Hz}, 1 \mathrm{H}) ; 5.22(\mathrm{dt}, J=1.6,17.1 \mathrm{~Hz}, 1 \mathrm{H}) ; 5.08(\mathrm{dt}, J=1.5$, $10.2 \mathrm{~Hz}, 1 \mathrm{H}) ; 2.34$ (s, 3H); $0.95(\mathrm{t}, J=8.0 \mathrm{~Hz}, 9 \mathrm{H}) ; 0.61(\mathrm{dq}, J=2.8,7.5 \mathrm{~Hz}, 6 \mathrm{H})$.

${ }^{13} \mathrm{C}$ NMR (100 MHz, $\left.\mathrm{CDCl}_{3}, \delta\right): 141.9,140.7,134.4,130.3,127.1,126.5,126.3,113.7,73.1$, 19.4, 7.0, 5.1.

IR (NaCl, thin film): 2955, 2877, 1639, 1461, 1066, 1007, 744.

HRMS-ESI (m / z): [M + Na $]^{+}$calcd for $\mathrm{C}_{16} \mathrm{H}_{26} \mathrm{OSiNa}$, 285.165; found, 285.165. 


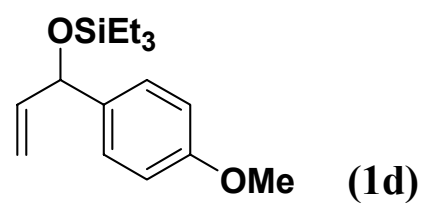

The reaction of ethylene and $p$-anisaldehyde $(61 \mu \mathrm{L}, 0.5 \mathrm{mmol})$ with $\mathrm{Ni}(\operatorname{cod})_{2}$, tris-o-methoxyphenylphosphine and TESOTf $(197 \mu \mathrm{L}, 0.875 \mathrm{mmol})$, triethylamine in toluene following the general procedure 1 above, afforded $\mathbf{1 d}$ in $95 \%$ isolated yield as a colorless oil.

${ }^{1} \mathrm{H}$ NMR $\left(400 \mathrm{MHz}, \mathrm{CDCl}_{3}, \delta\right): 7.30(\mathrm{~d}, J=8.7 \mathrm{~Hz}, 2 \mathrm{H}) ; 6.90(\mathrm{~d}, J=8.7 \mathrm{~Hz}, 2 \mathrm{H}) ; \quad 5.97$ (ddd, $J$ $=5.9,10.2,16.9 \mathrm{~Hz}, 1 \mathrm{H}) ; 5.29(\mathrm{dt}, J=1.4,17.0 \mathrm{~Hz}, 1 \mathrm{H}) ; 5.16(\mathrm{~d}, J=5.9 \mathrm{~Hz}, 1 \mathrm{H}) ; 5.10(\mathrm{dt}, J=$ $1.4,10.2 \mathrm{~Hz}, 1 \mathrm{H}) ; 3.83(\mathrm{~s}, 3 \mathrm{H}) ; 0.96(\mathrm{t}, J=7.9 \mathrm{~Hz}, 9 \mathrm{H}) ; 0.63(\mathrm{dq}, J=1.8,7.5 \mathrm{~Hz}, 6 \mathrm{H})$.

${ }^{13} \mathrm{C} \mathrm{NMR}\left(100 \mathrm{MHz}, \mathrm{CDCl}_{3}, \delta\right): 158.9,142.0,136.2,127.4,113.7,113.4,75.4,55.4,7.0,5.1$. IR ( $\mathrm{NaCl}$, thin film): 2955, 2877, 1639, 1511, 1464, 1246, 1037, 744.

HRMS-ESI ( $\mathrm{m} / \mathrm{z}$ ): $[\mathrm{M}+\mathrm{Na}]^{+}$calcd for $\mathrm{C}_{16} \mathrm{H}_{26} \mathrm{O}_{2} \mathrm{SiNa}$, 301.159; found, 301.159 .

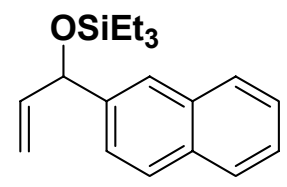

(1e)

The reaction of ethylene and 2-naphthaldehyde $(78.1 \mathrm{mg}, 0.5 \mathrm{mmol})$ with $\mathrm{Ni}(\operatorname{cod})_{2}$, tris-o-methoxyphenylphosphine and TESOTf $(197 \mu \mathrm{L}, 0.875 \mathrm{mmol})$, triethylamine in toluene following the general procedure 2 above, afforded 1e in $95 \%$ isolated yield as a colorless oil.

${ }^{1} \mathrm{H}$ NMR (400 MHz, $\mathrm{CDCl}_{3}, \delta$ ): $7.82-7.92(\mathrm{~m}, 4 \mathrm{H}) ; 7.48-7.55$ (m, 3H); 6.07 (ddd, $J=6.2$, $10.2,15.8 \mathrm{~Hz}, 1 \mathrm{H}) ; 5.35-5.45$ (m, 2H); 5.17 (dt, $J=1.3,10.1 \mathrm{~Hz}, 1 \mathrm{H}) ; 1.00$ (t, $J=7.8 \mathrm{~Hz}, 9 \mathrm{H})$; $0.68(\mathrm{dq}, J=2.5,7.5 \mathrm{~Hz}, 6 \mathrm{H})$.

${ }^{13} \mathrm{C}$ NMR $\left(100 \mathrm{MHz}, \mathrm{CDCl}_{3}, \delta\right): 141.7,141.4,133.5,133.0,128.2,128.1,127.7,126.1,125.8$, 124.8, 124.6, 114.0, 76.0, 7.0, 5.1.

IR (NaCl, thin film): 2955, 2876, 1640, 1458, 1239, 1006, 743.

HRMS-ESI (m / z): [M + Na] $]^{+}$calcd for $\mathrm{C}_{19} \mathrm{H}_{26} \mathrm{OSiNa}, 321.165$; found, 321.164 . 


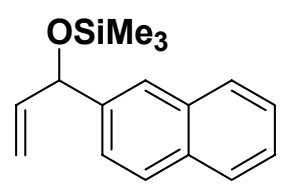

(1f)

The reaction of ethylene and 2-naphthaldehyde $(78.1 \mathrm{mg}, 0.5 \mathrm{mmol})$ with $\mathrm{Ni}(\mathrm{cod})_{2}$, tris-o-methoxyphenyl- phosphine and TMSOTf $(158 \mu \mathrm{L}, 0.875 \mathrm{mmol})$, triethylamine in toluene following the general procedure 2 above, afforded $\mathbf{1 f}$ in $60 \%$ isolated yield as a colorless oil.

${ }^{1} \mathrm{H}$ NMR (400 MHz, $\left.\mathrm{CDCl}_{3}, \delta\right): 7.80-7.90(\mathrm{~m}, 4 \mathrm{H}) ; 7.45-7.54(\mathrm{~m}, 3 \mathrm{H}) ; 6.06$ (ddd, $J=5.6$, $10.2,17.4 \mathrm{~Hz}, 1 \mathrm{H}) ; 5.30(\mathrm{dt}, J=1.5,17.3 \mathrm{~Hz}, 1 \mathrm{H}) ; 5.37$ (bs, $1 \mathrm{H}) ; 5.17(\mathrm{dt}, J=1.4,10.2 \mathrm{~Hz}, 1 \mathrm{H})$; $0.18(\mathrm{~s}, 9 \mathrm{H})$.

${ }^{13} \mathrm{C}$ NMR (100 MHz, $\left.\mathrm{CDCl}_{3}, \delta\right): 141.4,141.0,133.5,133.0,128.19,128.18,127.9,126.2,125.9$, $124.9,124.8,114.4,76.1,0.4$.

IR ( $\mathrm{NaCl}$, thin film): 2958, 1640, 1251, 1077, 841.

HRMS-ESI (m / z): [M + Na] $]^{+}$calcd for $\mathrm{C}_{16} \mathrm{H}_{20} \mathrm{OSiNa}$, 279.118; found, 279.119.

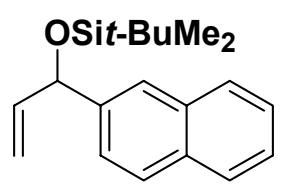

(1g)

The reaction of ethylene and 2-naphthaldehyde (78.1 mg, $0.5 \mathrm{mmol})$ with $\mathrm{Ni}(\mathrm{cod})_{2}$, tris-o-methoxy- phenylphosphine and TBSOTf $(201 \mu \mathrm{L}, 0.875 \mathrm{mmol})$, triethylamine in toluene following the general procedure 2 above, afforded $1 \mathrm{~g}$ in $67 \%$ isolated yield as a colorless oil.

${ }^{1} \mathrm{H}$ NMR (400 MHz, $\left.\mathrm{CDCl}_{3}, \delta\right): 7.80-7.92(\mathrm{~m}, 4 \mathrm{H}) ; 7.45-7.55(\mathrm{~m}, 3 \mathrm{H}) ; 6.04$ (ddd, $J=5.8$, $10.2,16.8 \mathrm{~Hz}, 1 \mathrm{H}) ; 5.39$ (dt, $J=1.5,17.0 \mathrm{~Hz}, 1 \mathrm{H}) ; 5.38(\mathrm{~s}, 1 \mathrm{H}) ; 5.14(\mathrm{dt}, J=1.5,10.2 \mathrm{~Hz}, 1 \mathrm{H})$; 0.99 (s, 9H); 0.16 (s, 3H); 0.06 (s, 3H).

${ }^{13} \mathrm{C}$ NMR (100 MHz, $\left.\mathrm{CDCl}_{3}, \delta\right): 141.8,141.4,133.5,133.0,128.2,128.1,127.9,126.1,125.8$, $124.8,124.6,113.8,76.2,26.1,18.6,-4.4,-4.6$.

IR (NaCl, thin film): 2956, 2857, 1636, 1472, 1252, 1081, 837.

HRMS-ESI (m / z): [M + Na] $]^{+}$calcd for $\mathrm{C}_{19} \mathrm{H}_{26} \mathrm{OSiNa}, 321.165$; found, 321.164 . 


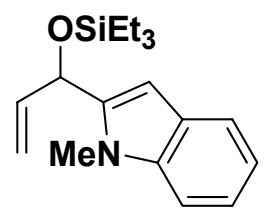

(1h)

The reaction of ethylene and 1-methyl-2-indolecarboxaldehyde (79.6 mg, $0.5 \mathrm{mmol})$ with $\mathrm{Ni}(\operatorname{cod})_{2}$, tris-o-methoxy- phenylphosphine and TESOTf $(197 \mu \mathrm{L}, 0.875 \mathrm{mmol})$, triethylamine in toluene following the general procedure 2 above, afforded $\mathbf{1 h}$ in $67 \%$ isolated yield as a colorless oil.

${ }^{1} \mathrm{H}$ NMR $\left(400 \mathrm{MHz}, \mathrm{CDCl}_{3}, \delta\right): 7.63(\mathrm{~d}, J=7.8 \mathrm{~Hz}, 1 \mathrm{H}) ; 7.36(\mathrm{~d}, J=8.2 \mathrm{~Hz}, 1 \mathrm{H}) ; 7.26(\mathrm{t}, J=$ $8.3 \mathrm{~Hz}, 1 \mathrm{H}) ; 7.14(\mathrm{t}, J=7.9 \mathrm{~Hz}, 1 \mathrm{H}) ; 6.43(\mathrm{~s}, 1 \mathrm{H}) ; 6.13(\mathrm{ddd}, J=4.5,10.3,17.1 \mathrm{~Hz}, 1 \mathrm{H}) ; 5.52$ (ddd, $J=1.7,1.7,4.5 \mathrm{~Hz}, 1 \mathrm{H}) ; 5.39$ (ddd, $J=1.7,1.7,17.1 \mathrm{~Hz}, 1 \mathrm{H}$ ); 5.25 (ddd, $J=1.7,1.7$, $10.4,1 \mathrm{H}) ; 3.82$ (s, 3H); 0.98 (t, $J=8.0 \mathrm{~Hz}, 9 \mathrm{H}) ; 0.66$ (dq, $J=1.4,8.0 \mathrm{~Hz}, 6 \mathrm{H})$.

${ }^{13} \mathrm{C}$ NMR (100 MHz, $\left.\mathrm{CDCl}_{3}, \delta\right): 140.6,139.7,138.5,127.5,121.5,120.8,119.4,114.9,109.1$, $100.5,70.4,31.0,7.0,5.0$.

IR (NaCl, thin film): 2955, 2911, 2876, 1911, 1758, 1641, 1469, 1238, 1009, 841, 731.

HRMS-ESI (m / z): [M + Na $]^{+}$calcd for $\mathrm{C}_{18} \mathrm{H}_{27} \mathrm{NOSiNa}$, 324.178; found, 324.178 .

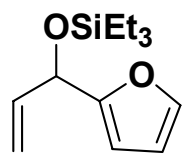

(1i)

The reaction of ethylene and furan-2-carbaldehyde (41 $\mu \mathrm{L}, 0.5 \mathrm{mmol})$ with $\mathrm{Ni}(\mathrm{cod})_{2}$, tris-o-methoxyphenylphosphine and TESOTf $(197 \mu \mathrm{L}, 0.875 \mathrm{mmol})$, triethylamine in toluene following the general procedure 1 above, afforded $1 \mathbf{i}$ in $38 \%$ isolated yield as a colorless oil.

${ }^{1} \mathrm{H}$ NMR (400 MHz, $\left.\mathrm{CDCl}_{3}, \delta\right): 7.37$ (bs, $\left.1 \mathrm{H}\right) ; 6.32(\mathrm{dd}, J=1.9,3.1 \mathrm{~Hz}, 1 \mathrm{H}) ; 6.22(\mathrm{~d}, J=3.2 \mathrm{~Hz}$, $1 \mathrm{H}) ; 6.06(\mathrm{~m}, 1 \mathrm{H}) ; 5.40$ (d, $J=17.1 \mathrm{~Hz}, 1 \mathrm{H}) ; 5.21$ (d, $J=7.9 \mathrm{~Hz}, 2 \mathrm{H}) ; 0.95$ (t, $J=7.9 \mathrm{~Hz}, 9 \mathrm{H})$; $0.63(\mathrm{q}, J=7.9 \mathrm{~Hz}, 6 \mathrm{H})$.

${ }^{13} \mathrm{C}$ NMR (100 MHz, $\left.\mathrm{CDCl}_{3}, \delta\right): 156.0,142.1,138.1,115.3,110.4,106.4$. 69.3, 6.9, 4.9.

IR (NaCl, thin film): 2956, 2878, 1646, 1459, 1237, 1010, 733.

HRMS-ESI ( $\mathrm{m} / \mathrm{z}$ ): [M + Na] $]^{+}$calcd for $\mathrm{C}_{13} \mathrm{H}_{22} \mathrm{O}_{2} \mathrm{SiNa}$, 261.128; found, 261.129. 


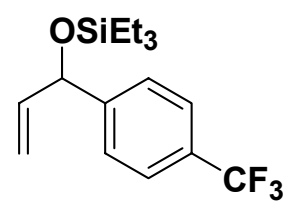

(1j)

The reaction of ethylene and 4-(trifluoromethyl)-benzaldehyde ( $70 \mu \mathrm{L}, 0.5 \mathrm{mmol})$ with $\mathrm{Ni}(\mathrm{cod})_{2}$, tris-o-methoxyphenylphosphine and TESOTf $(197 \mu \mathrm{L}, 0.875 \mathrm{mmol})$, triethylamine in toluene following the general procedure 1 above, afforded a mixture of $\mathbf{1} \mathbf{j}$ and triethylsilylethers of pinnacol coupling products. This mixture was subjected to TBAF to isolate $25 \%$ of the desilylated $\mathbf{1} \mathbf{j}$ as a colorless oil.

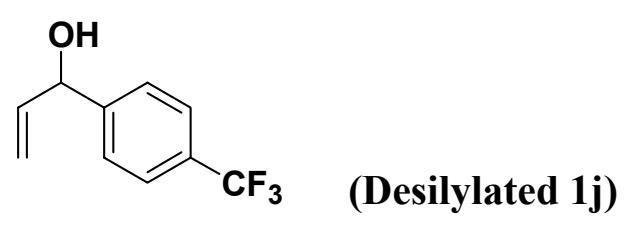

${ }^{1} \mathrm{H}$ NMR $\left(400 \mathrm{MHz}, \mathrm{CDCl}_{3}, \delta\right): 7.62(\mathrm{~d}, J=8.2 \mathrm{~Hz}, 2 \mathrm{H}) ; 7.50(\mathrm{~d}, J=8.4 \mathrm{~Hz}, 2 \mathrm{H}) ; 6.02$ (ddd, $J=$ $6.3,10.3,16.9 \mathrm{~Hz}, 1 \mathrm{H}$ ); 5.38 (ddd, $J=1.2,1.2,17.0 \mathrm{~Hz}, 1 \mathrm{H}$ ); 5.27 (bd, $J=7.0 \mathrm{~Hz}, 1 \mathrm{H}$ ); 5.25 (ddd, $J=1.2,1.2,10.3 \mathrm{~Hz}, 1 \mathrm{H}) ; 2.10$ (bs, $1 \mathrm{H}$ ).

${ }^{13} \mathrm{C}$ NMR $\left(100 \mathrm{MHz}, \mathrm{CDCl}_{3}, \delta\right): 146.5,139.8,130.0(J=32.3 \mathrm{~Hz}), 126.7,125.7,123.0,116.4$, 75.1.

$\left.19 \mathrm{~F} \mathrm{NMR} \mathrm{(376} \mathrm{MHz,} \mathrm{CDCl}_{3}, \delta\right):-66.8(\mathrm{~s}, 3 \mathrm{~F})$.

IR (NaCl, thin film): 3342, 1620, 1419, 1328, 1166, 1126, 1068, 931.

HRMS-ESI (m / z): [M + Na] $]^{+}$calcd for $\mathrm{C}_{10} \mathrm{H}_{9} \mathrm{OF}_{3} \mathrm{Na}, 202.060$; found, 202.059 . 


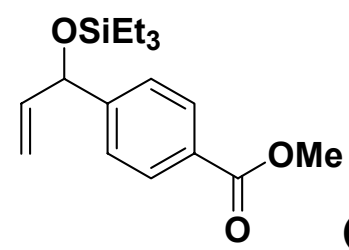

(1k)

The reaction of ethylene and methyl-4-formyl-benzoate $(88 \mathrm{mg}, 0.536 \mathrm{mmol})$ with $\mathrm{Ni}(\mathrm{cod})_{2}$, tris-o-methoxy- phenylphosphine and TESOTf $(197 \mu \mathrm{L}, 0.875 \mathrm{mmol})$, triethylamine in toluene following the general procedure 2 above, afforded $1 \mathbf{k}$ in $34 \%$ isolated yield as a colorless oil.

${ }^{1} \mathrm{H}$ NMR $\left(400 \mathrm{MHz}, \mathrm{CDCl}_{3}, \delta\right): 8.01(\mathrm{~d}, J=8.4 \mathrm{~Hz}, 2 \mathrm{H}) ; 7.43(\mathrm{~d}, J=8.1 \mathrm{~Hz}, 2 \mathrm{H}) ; 5.92$ (ddd, $J=$ $6.0,10.2,16.9 \mathrm{~Hz}, 1 \mathrm{H}) ; 5.31$ (ddd, $J=1.5,1.5,17.0 \mathrm{~Hz}, 1 \mathrm{H}$ ); 5.21 (bd, $J=6.0 \mathrm{~Hz}, 1 \mathrm{H}) ; 5.11$ (ddd, $J=1.4,1.4,10.2 \mathrm{~Hz}, 1 \mathrm{H}) ; 3.91(\mathrm{~s}, 3 \mathrm{H}) ; 0.93$ (t, $J=7.8 \mathrm{~Hz}, 9 \mathrm{H}) ; 0.61$ (dq, $J=1.7,7.5 \mathrm{~Hz}$, $6 \mathrm{H})$.

${ }^{13} \mathrm{C}$ NMR $\left(100 \mathrm{MHz}, \mathrm{CDCl}_{3}, \delta\right): 167.2,149.1,141.1,129.8,129.1,126.1,114.5,75.6,52.2,6.9$, 5.0.

IR (NaCl, thin film): 2954, 2912, 2877, 1727, 1610, 1436, 1278, 1113, 1019, 842, 745.

HRMS-ESI (m / z): [M + Na $]^{+}$calcd for $\mathrm{C}_{17} \mathrm{H}_{26} \mathrm{O}_{3} \mathrm{SiNa}, 329.154$; found, 329.155 .

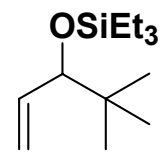

(11)

The reaction of ethylene and pivaldehyde $(55 \mu \mathrm{L}, 0.5 \mathrm{mmol})$ with $\mathrm{Ni}(\mathrm{cod})_{2}$, tris-o-methoxyphenylphosphine and TESOTf $(197 \mu \mathrm{L}, 0.875 \mathrm{mmol})$, triethylamine in toluene following the general procedure 1 above, afforded 11 in $70 \%$ isolated yield as a colorless oil.

${ }^{1} \mathrm{H}$ NMR (400 MHz, $\mathrm{CDCl}_{3}, \delta$ ): 5.97 (ddd, $\left.J=5.9,10.2,16.9 \mathrm{~Hz}, 1 \mathrm{H}\right) ; 5.11(\mathrm{~d}, J=8.5 \mathrm{~Hz}, 1 \mathrm{H})$; 5.08 (bs, $1 \mathrm{H}$ ); 3.67 (d, $J=7.5 \mathrm{~Hz}, 1 \mathrm{H}) ; 0.96$ (t, $J=7.9 \mathrm{~Hz}, 9 \mathrm{H}) ; 0.86$ (s, 9H); 0.63 (q, $J=7.7 \mathrm{~Hz}$, $6 \mathrm{H})$.

${ }^{13} \mathrm{C}$ NMR (100 MHz, $\left.\mathrm{CDCl}_{3}, \delta\right): 139.4,115.8,82.4,35.5,26.0,7.2,5.3$.

IR (NaCl, thin film): 2955, 2877, 1641, 1462, 1239, 1082, 835. 


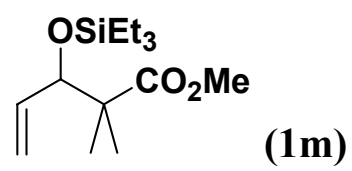

The reaction of ethylene and 2,2-dimethyl-3-oxo-propionic acid methyl ester (70 $\mathrm{mg}, 0.54 \mathrm{mmol}$ ) with $\mathrm{Ni}(\mathrm{cod})_{2}$, tris-o-methoxyphenylphosphine and TESOTf $(197 \mu \mathrm{L}, 0.875 \mathrm{mmol})$, triethylamine in toluene following the general procedure 1 above, afforded $\mathbf{1 m}$ in $81 \%(0.28$ mmol) isolated yield as a colorless oil.

${ }^{1} \mathrm{H}$ NMR (400 MHz, $\mathrm{CDCl}_{3}, \delta$ ): 5.75 (ddd, $\left.J=7.6,10.4,17.5 \mathrm{~Hz}, 1 \mathrm{H}\right) ; 5.17$ (bd, $J=17.3 \mathrm{~Hz}$, $1 \mathrm{H}) ; 5.15$ (bd, $J=10.3 \mathrm{~Hz}, 1 \mathrm{H}) ; 4.31$ (d, $J=7.6 \mathrm{~Hz}, 1 \mathrm{H}) ; 3.66$ (s, 3H); 1.15 (s, 3H); 1.05 (s, 3H); $0.92(\mathrm{t}, J=7.9 \mathrm{~Hz}, 9 \mathrm{H}) ; 0.55$ (dq, $J=1.5,7.6 \mathrm{~Hz}, 6 \mathrm{H})$.

${ }^{13} \mathrm{C} \mathrm{NMR}\left(100 \mathrm{MHz}, \mathrm{CDCl}_{3}, \delta\right): 177.4,137.8,117.3,79.2,51.8,48.3,21.4,19.9,7.0,5.2$.

IR (NaCl, thin film): 2954, 2878, 1745, 1732, 1642, 1468, 1261, 1087, 834.

HRMS-ESI (m / z): [M + Na $]^{+}$calcd for $\mathrm{C}_{14} \mathrm{H}_{28} \mathrm{O}_{3} \mathrm{SiNa}$, 295.170; found, 295.171 .

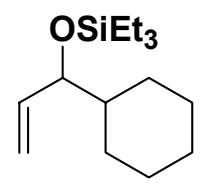

(1n)

The reaction of ethylene and cyclohexanecarboxaldehyde $(60 \mu \mathrm{L}, 0.5 \mathrm{mmol})$ with $\mathrm{Ni}(\mathrm{cod})_{2}$, tris-o-methoxyphenylphosphine and TESOTf $(197 \mu \mathrm{L}, 0.875 \mathrm{mmol})$, triethylamine in toluene following the general procedure 1 above, afforded $\mathbf{1} \mathbf{n}$ in $25 \%$ yield as determined by ${ }^{1} \mathrm{H}$ NMR versus a standard. Another experiment was carried out under $2 \mathrm{~atm}$ of ethylene and yielded $34 \%$ 1n and $66 \%$ silyl enol ether of cyclohexanecarboxaldehyde. Treatment of this mixture with a TBAF / THF / $\mathrm{H}_{2} \mathrm{O}$ solution removed the silyl enol ether from the mixture and column chromatography isolated $\mathbf{1 n}$ as a colorless oil.

${ }^{1} \mathrm{H} \mathrm{NMR}\left(400 \mathrm{MHz}, \mathrm{CDCl}_{3}, \delta\right): 5.78(\mathrm{ddd}, J=7.0,10.3,17.3 \mathrm{~Hz}, 1 \mathrm{H}) ; 5.07$ (m, 2H); $3.78(\mathrm{t}, J=$ $6.6 \mathrm{~Hz}, 1 \mathrm{H}) ; 1.40-0.90(\mathrm{~m}, 11 \mathrm{H}) ; 0.95(\mathrm{t}, J=8.0 \mathrm{~Hz}, 9 \mathrm{H}) ; 0.59$ (q, $J=8.0 \mathrm{~Hz}, 6 \mathrm{H})$.

${ }^{13} \mathrm{C}$ NMR (125 MHz, $\left.\mathrm{CDCl}_{3}, \delta\right): 140.7,114.8,78.9,44.5,29.0,29.0,26.9,26.5,26.5,7.1,5.2$. IR (NaCl, thin film): 2953, 2926, 2877, 1644, 1451, 1239, 1068, 743.

HRMS-ESI (m / z): [M + Na] $]^{+}$calcd for $\mathrm{C}_{15} \mathrm{H}_{30} \mathrm{OSiNa}$, 277.196; found, 277.197. 
Nickel-catalyzed coupling of monosubstituted olefins and aldehydes (2a - 2p).

\section{Nickel-catalyzed coupling of monosubstituted alkenes and aldehydes (homoallylic products)}

General procedure 3. A $10 \mathrm{~mL}$ test tube and a stir bar were oven-dried and brought into a glove box. Ni(cod) $2(27.5 \mathrm{mg}, 0.1 \mathrm{mmol}, 20 \mathrm{~mol} \%)$ and ligand $(0.2 \mathrm{mmol}, 40 \mathrm{~mol} \%$ as specified $)$ were added to the test tube, the test tube was sealed with a septum, and the sealed tube was brought out of the glove box and connected to an argon line. The catalyst mixture was dissolved in toluene $(2.5 \mathrm{~mL})$ under argon and stirred $5 \mathrm{~min}$ at room temperature. Alkene $(0.5 \mathrm{~mL})$, triethylamine $(418 \mu \mathrm{L}, 3 \mathrm{mmol}, 600 \mathrm{~mol} \%)$ and then aldehyde $(0.5 \mathrm{mmol}, 100 \mathrm{~mol} \%)$ were added. TESOTf (197 $\mu \mathrm{L}, 0.875 \mathrm{mmol}, 175 \mathrm{~mol} \%)$ was added. The mixture was stirred at room temperature for $48 \mathrm{~h}$. The mixture was filtered through a plug of silica gel. Solvent was removed under reduced pressure and the crude mixture was diluted in hexane. Purification via flash chromatography on silica afforded the coupling product.<smiles>C=CCC(OCC)c1ccccc1</smiles><smiles>C=C(C)C(OCC)c1ccccc1</smiles>

A $10 \mathrm{~mL}$ test tube and a stir bar were oven-dried and brought into a glove box. Ni(cod $)_{2}(27.5$ $\mathrm{mg}, 0.1 \mathrm{mmol}, 20 \mathrm{~mol} \%)$ and $\mathrm{EtOPh}_{2} \mathrm{P}(43 \mu \mathrm{l}, 0.2 \mathrm{mmol}, 40 \mathrm{~mol} \%)$ were added to the round bottom flask, the flask was sealed with a septum, and the sealed flask was brought out of the glove box and connected to an argon line. The catalyst mixture was dissolved in toluene $(2.5 \mathrm{~mL})$ under argon and stirred $5 \mathrm{~min}$ at room temperature. The reaction mixture was purged with propene for $1 \mathrm{~min}$ to remove argon, taken care not to introduce oxygen. The propene atmosphere was maintained with a propene balloon. Triethylamine $(418 \mu \mathrm{L}, 3 \mathrm{mmol}, 600 \mathrm{~mol} \%)$ was added. benzaldehyde $(51 \mu \mathrm{L}, 0.5 \mathrm{mmol}, 100 \mathrm{~mol} \%)$ was added. Silyltriflate $(0.875 \mathrm{mmol}, 175 \mathrm{~mol} \%$, as specified) was added. The mixture was stirred at room temperature for $48 \mathrm{~h}$. The mixture was filtered through a plug of silica gel. Solvent was removed under reduced pressure and ${ }^{1} \mathrm{H}$ NMR of the crude mixture indicated the total yield of $\mathbf{2} \mathbf{a}$ and $\mathbf{2} \mathbf{a}^{\prime}$ was $73 \%$ and the ratio of $\mathbf{2 a}: \mathbf{2} \mathbf{a}^{\prime}$ is 89:11. Purification via flash chromatography on silica afforded $\mathbf{2 a}$ and $\mathbf{2} \mathbf{a}^{\prime}$ as colorless oils. 


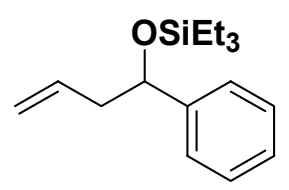

(2a)

${ }^{1} \mathrm{H}$ NMR (400 MHz, $\left.\mathrm{CDCl}_{3}, \delta\right)$ : 7.27-7.38 (m, 5H); 5.78-5.89 (m, 1H); 5.05-5.10 (m, 2H); 4.74 $(\mathrm{dd}, J=7.2,5.5 \mathrm{~Hz}, 1 \mathrm{H}) ; 2.42-2.59(\mathrm{~m}, 2 \mathrm{H}) ; 0.94(\mathrm{t}, J=7.9 \mathrm{~Hz}, 9 \mathrm{H}) ; 0.59$ (dq, $J=2.6,7.9 \mathrm{~Hz}$, $6 \mathrm{H})$.

${ }^{13} \mathrm{C}$ NMR (100 MHz, $\left.\mathrm{CDCl}_{3}, \delta\right): 145.3,135.4,128.2,127.2,126.1,117.0,75.1,45.6,7.0,5.0$. IR (NaCl, thin film): 2954, 2927, 2876, 1644, 1493, 1449, 1239, 1090, 858, 699. HRMS-ESI (m / z): [M + Na] $]^{+}$calcd for $\mathrm{C}_{16} \mathrm{H}_{26} \mathrm{OSiNa}$, 285.165; found, 285.163 .

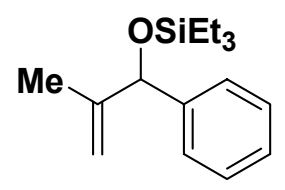

\section{(2a')}

${ }^{1} \mathrm{H}$ NMR (400 MHz, $\mathrm{CDCl}_{3}, \delta$ ): 7.24-7.39 (m, 5H); 5.15 (m, 2H); 4.86 (s, 1H); 1.56 (s, 3H); 0.94 (t, $J=7.8 \mathrm{~Hz}, 9 \mathrm{H}) ; 0.61$ (q, $J=7.8 \mathrm{~Hz}, 6 \mathrm{H})$.

${ }^{13} \mathrm{C}$ NMR (100 MHz, $\left.\mathrm{CDCl}_{3}, \delta\right): 148.1,143.5,128.1,127.0,126.3,111.0,78.4,17.4,7.0,5.0$. IR (NaCl, thin film): 2955, 2913, 2877, 1451, 1237, 1091, 1066, 1005, 899, 853, 740, 698. HRMS-ESI (m / z): [M + Na] ${ }^{+}$calcd for $\mathrm{C}_{16} \mathrm{H}_{26} \mathrm{OSiNa}$, 285.165; found, 285.165. 
<smiles>CCOC(C/C=C/c1ccccc1)c1ccccc1</smiles>

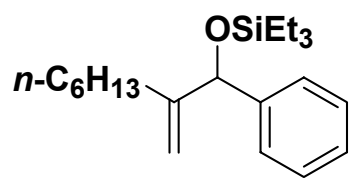

\section{$\left(\mathbf{2} \mathbf{b}^{\prime}\right)$}

The reaction of 1-octene and benzaldehyde $(51 \mu \mathrm{L}, 0.5 \mathrm{mmol})$ with $\mathrm{Ni}(\operatorname{cod})_{2}, \mathrm{EtOPh}_{2} \mathrm{P}(43 \mu \mathrm{l}$, $0.2 \mathrm{mmol}, 40 \mathrm{~mol} \%)$ and TESOTf $(197 \mu \mathrm{L}, 0.875 \mathrm{mmol})$, triethylamine in toluene following the general procedure 3 above afforded $\mathbf{2} \mathbf{b}$ and $\mathbf{2} \mathbf{b}^{\prime}$ in $85 \%$ total yield according to ${ }^{1} \mathrm{H}$ NMR of the crude mixture and the ratio of $\mathbf{2} \mathbf{b}: \mathbf{2} \mathbf{b}$ ' is $95: 5$. The $E / Z$ ratio of $\mathbf{2 b}$ is $\mathbf{7 5 : 2 5}$. Purification via flash chromatography on silica afforded $\mathbf{2} \mathbf{b}$ and $\mathbf{2} \mathbf{b}^{\prime}$ as colorless oils.

In another experiment, the reaction of 1-octene $(1 \mathrm{~mL})$ and benzaldehyde $(51 \mu \mathrm{L}, 0.5 \mathrm{mmol})$ with $\mathrm{Ni}(\operatorname{cod})_{2}, \mathrm{Cy}_{2} \mathrm{PhP}$ (56 mg, $0.2 \mathrm{mmol}, 40 \mathrm{~mol} \%$ ) and TESOTf (197 $\left.\mu \mathrm{L}, 0.875 \mathrm{mmol}\right)$, triethylamine in toluene following the general procedure 3 above afforded $\mathbf{2} \mathbf{b}^{\prime}$ and $\mathbf{2 b}$ in $73 \%$ total yield according to ${ }^{1} \mathrm{H}$ NMR of the crude mixture and the ratio of $\mathbf{2} \mathbf{b}^{\prime}: \mathbf{2} \mathbf{b}$ is $71: 29$. Purification via flash chromatography on silica afforded $\mathbf{2} \mathbf{b}^{\prime}$ and $\mathbf{2 b}$ in $70 \%$ isolated yield as a colorless oil.<smiles>CCOC(C/C=C/c1ccccc1)c1ccccc1</smiles>

(2b)

${ }^{1} \mathrm{H}$ NMR (400 MHz, $\left.\mathrm{CDCl}_{3}, \delta\right): 7.20-7.40$ (m, 5H); 5.30-5.50 (m, 2H); 4.63 (dd, J= 5.6, 7.2 Hz, $1 \mathrm{H}$ ); 2.45 (quintet, $J=6.1 \mathrm{~Hz}, 1 \mathrm{H}) ; 2.35$ (quintet, $J=5.9 \mathrm{~Hz}, 1 \mathrm{H}) ; 1.33(\mathrm{~m}, 2 \mathrm{H}) ; 0.92(\mathrm{t}, J=7.8$ $\mathrm{Hz}, 12 \mathrm{H}) ; 0.56(\mathrm{dq}, J=2.4,7.6 \mathrm{~Hz}, 6 \mathrm{H})$.

${ }^{13} \mathrm{C}$ NMR (100 MHz, $\left.\mathrm{CDCl}_{3}, \delta\right): 145.6,133.3,128.1,127.1,126.6,126.2,75.6,44.5,32.8$, 31.6, 29.3, 22.8, 14.2, 7.0, 5.1.

HRMS-ESI (m / z): $[\mathrm{M}+\mathrm{Na}]^{+}$calcd for $\mathrm{C}_{21} \mathrm{H}_{36} \mathrm{OSiNa}$, 355.243; found, 355.244. 


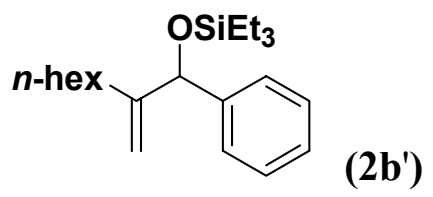

${ }^{1} \mathrm{H}$ NMR (400 MHz, $\left.\mathrm{CDCl}_{3}, \delta\right): 7.36(\mathrm{~d}, J=7.0 \mathrm{~Hz}, 2 \mathrm{H}) ; 7.31(\mathrm{t}, J=7.1 \mathrm{~Hz}, 2 \mathrm{H}) ; 7.24(\mathrm{t}, J=7.2$, $1 \mathrm{H}) ; 5.22$ (bs, 1H); 5.15 (bs, 1H); 4.87 (s, 1H); 1.96 (pentet, $J=7.8 \mathrm{~Hz}, 1 \mathrm{H}) ; 1.76$ (pentet, $J=$ $8.0 \mathrm{~Hz}, 1 \mathrm{H}) ; 1.15-1.40(\mathrm{~m}, 8 \mathrm{H}) ; 0.93$ (t, $J=8.0 \mathrm{~Hz}, 9 \mathrm{H}) ; 0.87$ (t, $J=6.8 \mathrm{~Hz}, 3 \mathrm{H}) ; 0.60$ (dq, $J=$ 1.6, $7.9 \mathrm{~Hz}, 6 \mathrm{H})$.

${ }^{13} \mathrm{C} \mathrm{NMR}\left(100 \mathrm{MHz}, \mathrm{CDCl}_{3}, \delta\right): 152.3,143.8,128.1,127.1,126.6,109.5,78.3,32.0,30.8,29.4$, 28.0, 22.8, 14.3, 7.0, 5.1.

IR (NaCl, thin film): 2956, 2876, 1647, 1456, 1089, 1066, 742.

HRMS-ESI (m / z): [M + Na $]^{+}$calcd for $\mathrm{C}_{21} \mathrm{H}_{36} \mathrm{OSiNa}$, 355.243; found, 355.242. 


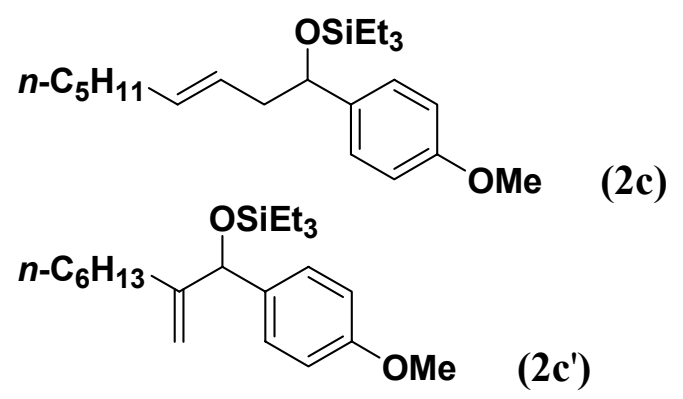

The reaction of 1-octene and 4-anisaldehyde $(61 \mu \mathrm{L}, 0.5 \mathrm{mmol})$ with $\mathrm{Ni}(\mathrm{cod})_{2}, \mathrm{EtOPh}_{2} \mathrm{P}(43 \mu 1$, $0.2 \mathrm{mmol}, 40 \mathrm{~mol} \%)$ and TESOTf $(197 \mu \mathrm{L}, 0.875 \mathrm{mmol})$, triethylamine in toluene following the general procedure 3 above afforded $2 \mathbf{c}$ and $2 \mathbf{c}^{\prime}$ in $85 \%$ total yield according to ${ }^{1} \mathrm{H}$ NMR of the crude mixture and the ratio of $\mathbf{2 c}: 2 \mathbf{c}$ is $>95: 5$. The $E / Z$ ratio of $\mathbf{2 c}$ is $75: 25$. Purification via flash chromatography on silica afforded $\mathbf{2 c}$ as a colorless oil. $\mathbf{2} \mathbf{c}^{\prime}$ was not detected.

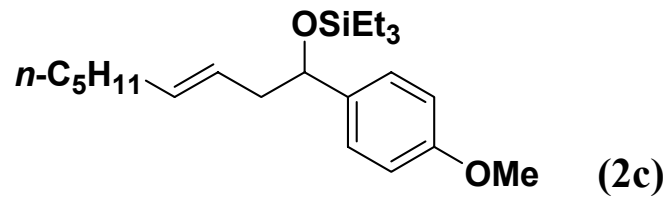

${ }^{1} \mathrm{H}$ NMR (400 MHz, $\left.\mathrm{CDCl}_{3}, \delta\right): 7.22(\mathrm{~d}, J=8.6 \mathrm{~Hz}, 2 \mathrm{H}) ; 6.84(\mathrm{~d}, J=8.6 \mathrm{~Hz}, 2 \mathrm{H}) ; 5.33-5.43$ (m, $2 \mathrm{H}) ; 4.58(\mathrm{dd}, J=6.1 \mathrm{~Hz}, 6.1 \mathrm{~Hz}, 1 \mathrm{H}) ; 3.81(\mathrm{~s}, 3 \mathrm{H}) ; 2.27-2.42$ (m, 2H); 1.93-1.98 (m, 2H); $1.22-1.60(\mathrm{~m}, 6 \mathrm{H}) ; 0.95(\mathrm{t}, J=8.0 \mathrm{~Hz}, 3 \mathrm{H}) ; 0.88(\mathrm{t}, J=7.8 \mathrm{~Hz}, 9 \mathrm{H}) ; 0.53(\mathrm{q}, J=7.8 \mathrm{~Hz}, 6 \mathrm{H})$.

${ }^{13} \mathrm{C}$ NMR (100 MHz, $\left.\mathrm{CDCl}_{3}, \delta\right): 158.7,137.9,133.2,127.3,126.7,113.4,75.2,55.4,44.5,32.8$, 31.6, 29.3, 22.8, 14.3, 7.0, 5.0.

IR (NaCl, thin film): 2955, 2876, 1613, 1512, 1459, 1302, 1247, 1172, 1078, 1005, 972, 830, 742.

HRMS-ESI (m / z): $[\mathrm{M}+\mathrm{Na}]^{+}$calcd for $\mathrm{C}_{22} \mathrm{H}_{38} \mathrm{O}_{2} \mathrm{SiNa}$, 385.2539; found, 385.2537. 


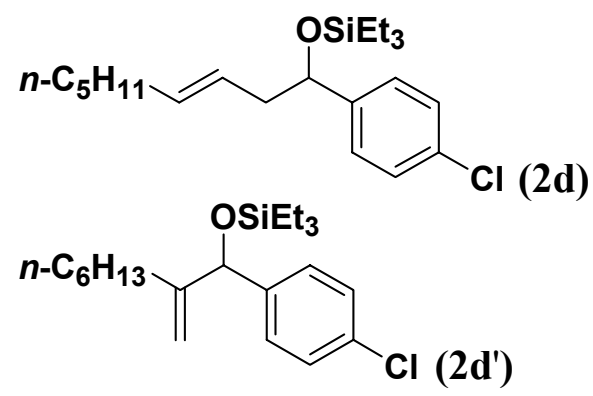

The reaction of 1-octene and 4-chlorobenzaldehyde (70 mg, $0.5 \mathrm{mmol})$ with $\mathrm{Ni}(\operatorname{cod})_{2}, \mathrm{EtOPh}_{2} \mathrm{P}$ (43 $\mu \mathrm{l}, 0.2 \mathrm{mmol}, 40 \mathrm{~mol} \%)$ and TESOTf $(197 \mu \mathrm{L}, 0.875 \mathrm{mmol})$, triethylamine in toluene following the general procedure 3 above afforded $\mathbf{2 d}$ and $\mathbf{2 d}$ ' in $37 \%$ total yield according to ${ }^{1} \mathrm{H}$ NMR of the crude mixture and the ratio of $\mathbf{2 d : 2 d}$ ' is $>95: 5$. The $E / Z$ ratio of $\mathbf{2 d}$ is 74:26. Purification via flash chromatography on silica afforded $\mathbf{2 d}$ as a colorless oil. $\mathbf{2 d}$ ' was not detected.

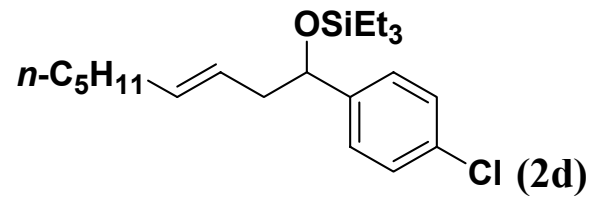

${ }^{1} \mathrm{H}$ NMR (400 MHz, $\left.\mathrm{CDCl}_{3}, \delta\right): 7.24(\mathrm{~m}, 4 \mathrm{H}) ; 5.30-5.41(\mathrm{~m}, 2 \mathrm{H}) ; 4.61(\mathrm{dd}, J=6.1 \mathrm{~Hz}, 6.1 \mathrm{~Hz}$, $1 \mathrm{H}) ; 2.26-2.40$ (m, 2H); 1.89-1.97 (m, 2H); 1.21-1.59 (m, 6H); 0.94 (t, $J=8.0 \mathrm{~Hz}, 3 \mathrm{H}) ; 0.89$ (t, $J$ $=7.8 \mathrm{~Hz}, 9 \mathrm{H}) ; 0.54(\mathrm{q}, J=7.8 \mathrm{~Hz}, 6 \mathrm{H})$.

${ }^{13} \mathrm{C}$ NMR (100 MHz, $\left.\mathrm{CDCl}_{3}, \delta\right): 144.1,133.7,132.5,128.2,127.5,126.0,74.8,44.4,32.8,31.5$, 29.2, 22.7, 14.3, 7.0, 4.9.

IR (NaCl, thin film): 2956, 2876, 1647, 1456, 1089, 1066, 742.

HRMS-ESI (m / z): [M + Na $]^{+}$calcd for $\mathrm{C}_{15} \mathrm{H}_{20} \mathrm{Na}, 223.1463$; found, 223.1305. 
<smiles>CCOC(C/C=C/c1ccccc1)c1ccc2ccccc2c1</smiles>

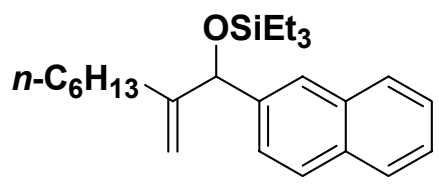

The reaction of 1-octene and 2-naphthaldehyde (78 mg, $0.5 \mathrm{mmol})$ with $\mathrm{Ni}(\mathrm{cod})_{2}, \mathrm{EtOPh}_{2} \mathrm{P}(43$ $\mu 1,0.2 \mathrm{mmol}, 40 \mathrm{~mol} \%)$ and TESOTf $(197 \mu \mathrm{L}, 0.875 \mathrm{mmol})$, triethylamine in toluene following the general procedure 3 above afforded $2 \mathrm{e}$ and $2 \mathrm{e}^{\prime}$ in $88 \%$ total yield according to ${ }^{1} \mathrm{H}$ NMR of the crude mixture and the ratio of $\mathbf{2 e}: 2 \mathbf{e}^{\prime}$ is $>95: 5$. The $E / Z$ ratio of $\mathbf{2 e}$ is $70: 30$. Purification via flash chromatography on silica afforded $\mathbf{2 e}$ as a colorless oil. $\mathbf{2} \mathbf{e}^{\prime}$ was not detected.

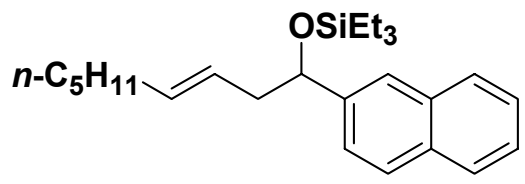

\section{(2e)}

${ }^{1} \mathrm{H}$ NMR (400 MHz, $\left.\mathrm{CDCl}_{3}, \delta\right):$ 7.83-7.92 (m, 3H); 7.80 (s, 1H); 7.48-7.59 (m, 3H); 5.43-5.53 $(\mathrm{m}, 2 \mathrm{H}) ; 4.89(\mathrm{dd}, J=6.9,13.2 \mathrm{~Hz}, 1 \mathrm{H}) ; 2.45-2.68(\mathrm{~m}, 2 \mathrm{H}) ; 1.98-2.05(\mathrm{~m}, 2 \mathrm{H}) ; 1.26-1.39$ (m, $6 \mathrm{H}) ; 0.97(\mathrm{t}, J=8.0 \mathrm{~Hz}, 9 \mathrm{H}) ; 0.94(\mathrm{t}, J=7.6 \mathrm{~Hz}, 3 \mathrm{H}) ; 0.63$ (q, $J=4.1,8.0 \mathrm{~Hz} 6 \mathrm{H})$.

${ }^{13} \mathrm{C}$ NMR $\left(100 \mathrm{MHz}, \mathrm{CDCl}_{3}, \delta\right): 143.1,133.4,133.0,132.3,128.1,127.9,126.5,126.0,125.6$, $125.6,124.7,124.7,75.7,44.4,32.8,31.7,29.3,22.8,14.3,7.0,5.1$.

IR (NaCl, thin film): 2956, 2929, 2875, 1458, 1414, 1377, 1239, 1086, 1005, 972, 744.

HRMS-ESI (m / z): $[\mathrm{M}+\mathrm{Na}]^{+}$calcd for $\mathrm{C}_{25} \mathrm{H}_{38} \mathrm{OSiNa}$, 405.2590; found, 405.2584. 


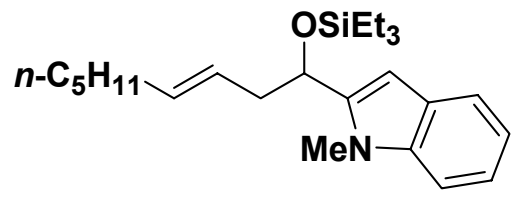

(2f)<smiles>C=C(CCCCCCCCCCCCCCCCCC)C(OCC)c1cc2ccccc2[nH]1</smiles>

(2f')

The reaction of 1-octene and 1-methyl-2-indolecarboxaldehyde (79.6 $\mathrm{mg}, 0.5 \mathrm{mmol})$ with $\mathrm{Ni}(\mathrm{cod})_{2}, \mathrm{EtOPh}_{2} \mathrm{P}(43 \mu \mathrm{l}, 0.2 \mathrm{mmol}, 40 \mathrm{~mol} \%)$ and TESOTf $(197 \mu \mathrm{L}, 0.875 \mathrm{mmol})$, triethylamine in toluene following the general procedure 3 above afforded $\mathbf{2 f}$ and $\mathbf{2} \mathbf{f}$ in $56 \%$ total yield according to ${ }^{1} \mathrm{H}$ NMR of the crude mixture and the ratio of $\mathbf{2 f : 2 f}$ ' is $>95: 5$. The $E / Z$ ratio of $\mathbf{2 f}$ is $83: 17$. Purification via flash chromatography on silica afforded $\mathbf{2 f}$ as a colorless oil. $2 \mathbf{f}^{\prime}$ was not detected.

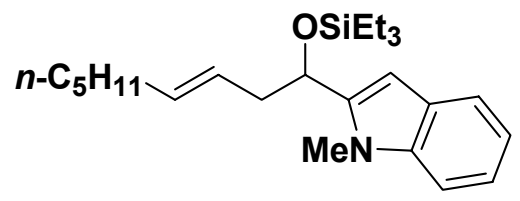

(2f)

${ }^{1} \mathrm{H}$ NMR (400 MHz, $\left.\mathrm{CDCl}_{3}, \delta\right): 7.65(\mathrm{~d}, J=7.8 \mathrm{~Hz}, 1 \mathrm{H}) ; 7.37(\mathrm{~d}, J=8.2 \mathrm{~Hz}, 1 \mathrm{H}) ; 7.27(\mathrm{t}, J=$ $7.1 \mathrm{~Hz}, 1 \mathrm{H}) ; 7.17(\mathrm{t}, J=7.1 \mathrm{~Hz}, 1 \mathrm{H}) ; 6.40(\mathrm{~s}, 1 \mathrm{H}) ; 5.43-5.59(\mathrm{~m}, 2 \mathrm{H}) ; 4.96(\mathrm{dd}, J=6.5,7.4 \mathrm{~Hz}$, $1 \mathrm{H}) ; 3.92(\mathrm{~s}, 3 \mathrm{H}) ; 2.56-2.71(\mathrm{~m}, 2 \mathrm{H}) ; 2.01-2.07(\mathrm{~m}, 2 \mathrm{H}) ; 1.29-1.42(\mathrm{~m}, 6 \mathrm{H}) ; 0.97(\mathrm{t}, J=8.0 \mathrm{~Hz}$, $9 \mathrm{H}) ; 0.95$ (t, $J=4.0 \mathrm{~Hz}, 3 \mathrm{H}) ; 0.63$ (dq, $J=1.1,8.0 \mathrm{~Hz}, 6 \mathrm{H})$.

${ }^{13} \mathrm{C}$ NMR (100 MHz, $\left.\mathrm{CDCl}_{3}, \delta\right): 142.3,138.4,133.7,127.7,126.2,121.3,120.7,119.4,109.1$, $100.2,70.6,42.2,32.8,31.6,31.0,29.3,22.8,14.3,7.0,5.0$.

IR (NaCl, thin film): 2954, 2927, 2874, 1466, 1339, 1236, 1072, 1010, 731.

HRMS-ESI (m / z): $[\mathrm{M}+\mathrm{Na}]^{+}$calcd for $\mathrm{C}_{24} \mathrm{H}_{39} \mathrm{ONSiNa}$, 408.2693; found, 408.2695. 
<smiles></smiles>

\section{(2g)}

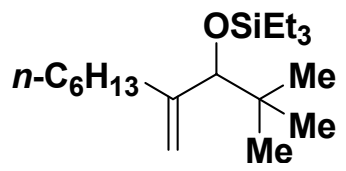

$\left(2 g^{\prime}\right)$

The reaction of 1-octene and pivaldehyde $(55 \mu \mathrm{L}, 0.5 \mathrm{mmol})$ with $\mathrm{Ni}(\mathrm{cod})_{2}, \mathrm{EtOPh}_{2} \mathrm{P}(43 \mu \mathrm{l}, 0.2$ mmol, $40 \mathrm{~mol} \%)$ and TESOTf $(197 \mu \mathrm{L}, 0.875 \mathrm{mmol})$, triethylamine in toluene following the general procedure 3 above afforded $\mathbf{2 g}$ and $\mathbf{2} \mathrm{g}^{\prime}$ in $64 \%$ total yield according to ${ }^{1} \mathrm{H}$ NMR of the

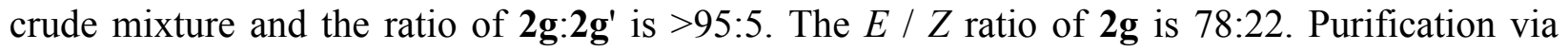
flash chromatography on silica afforded $\mathbf{2 g}$. $\mathbf{2} \mathbf{g}^{\prime}$ was not detected.

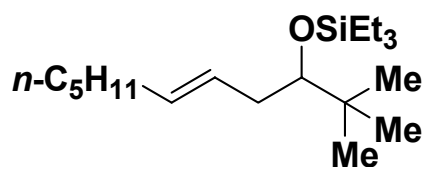

\section{(2g)}

${ }^{1} \mathrm{H}$ NMR (400 MHz, $\left.\mathrm{CDCl}_{3}, \delta\right): 5.37-5.53(\mathrm{~m}, 2 \mathrm{H}) ; 3.37$ (dd, $\left.J=3.8,7.4 \mathrm{~Hz}, 1 \mathrm{H}\right) ; 2.30-2.36(\mathrm{~m}$, $1 \mathrm{H}) ; 1.99-2.12(\mathrm{~m}, 3 \mathrm{H}) ; 1.27-1.42(\mathrm{~m}, 6 \mathrm{H}) ; 0.99$ (t, $J=8.0 \mathrm{~Hz}, 9 \mathrm{H}) ; 0.92$ (t, $J=6.8 \mathrm{~Hz}, 3 \mathrm{H})$; $0.90(\mathrm{~s}, 9 \mathrm{H}) ; 0.63(\mathrm{q}, J=8.0 \mathrm{~Hz}, 6 \mathrm{H})$.

${ }^{13} \mathrm{C} \mathrm{NMR}\left(100 \mathrm{MHz}, \mathrm{CDCl}_{3}, \delta\right): 130.6,128.5,81.2,36.2,31.8,31.4,29.5,27.6,26.5,22.8,14.2$, 7.3, 5.7.

IR (NaCl, thin film): 2956, 2876, 1466, 1238, 1096, 1009, 737.

HRMS-ESI (m / z): $[\mathrm{M}+\mathrm{Na}]^{+}$calcd for $\mathrm{C}_{19} \mathrm{H}_{40} \mathrm{OSiNa}$, 335.2746; found, 335.2741. 
<smiles>CCOC(C/C=C/c1ccccc1)c1ccccc1</smiles><smiles>C=C(Cc1ccccc1)C(OCC)c1ccccc1</smiles>

\section{$\left(2 h^{\prime}\right)$}

The reaction of allylbenzene and benzaldehyde $(51 \mu \mathrm{L}, 0.5 \mathrm{mmol})$ with $\mathrm{Ni}(\mathrm{cod})_{2}, \mathrm{Ph}_{3} \mathrm{P}(52 \mathrm{mg}$, $0.2 \mathrm{mmol}, 40 \mathrm{~mol} \%)$ and TESOTf $(197 \mu \mathrm{L}, 0.875 \mathrm{mmol})$, triethylamine in toluene following the general procedure 3 above afforded $\mathbf{2 h}$ and $\mathbf{2} \mathbf{h}$ ' in $86 \%$ total yield according to ${ }^{1} \mathrm{H}$ NMR of the crude mixture and the ratio of $\mathbf{2 h}: \mathbf{2 h}$ ' is $92: 8$. The $E / Z$ ratio of $\mathbf{2 h}$ is $>95: 5$. Purification via flash chromatography on silica afforded $\mathbf{2 h}$ as a colorless oil. $\mathbf{2} \mathbf{h}^{\prime}$ was subjected to TBAF and the free alcohol was isolated by flash chromatography on silica as a colorless oil.<smiles>CCOC(C/C=C/c1ccccc1)c1ccccc1</smiles>

(2h)

${ }^{1} \mathrm{H}$ NMR (400 MHz, $\left.\mathrm{CDCl}_{3}, \delta\right): 7.30-7.50(\mathrm{~m}, 10 \mathrm{H}) ; 6.51(\mathrm{~d}, J=15.9 \mathrm{~Hz}, 1 \mathrm{H}) ; 6.34(\mathrm{dt}, J=7.2$, $15.9 \mathrm{~Hz}, 1 \mathrm{H}) ; 4.89(\mathrm{dd}, J=5.3,7.2 \mathrm{~Hz}, 1 \mathrm{H}) ; 2.64-2.81(\mathrm{~m}, 2 \mathrm{H}) ; 1.03(\mathrm{t}, J=7.9 \mathrm{~Hz}, 9 \mathrm{H}) ; 0.68$ $(\mathrm{dq}, J=2.0,7.9 \mathrm{~Hz}, 6 \mathrm{H})$.

${ }^{13} \mathrm{C}$ NMR $\left(100 \mathrm{MHz}, \mathrm{CDCl}_{3}, \delta\right): 145.5,138.0,132.4,128.8,128.4,127.4,127.4,127.2,126.3$, 126.2, 75.5, 45.0, 7.1, 5.2.

IR (NaCl, thin film): 3062, 3028, 2955, 2911, 2876, 1600, 1494, 1453, 1414, 1239, 1088, 1070, 1006, 965, 830, 742, 700 .

HRMS-ESI (m / z): $[\mathrm{M}+\mathrm{Na}]^{+}$calcd for 361.1964; found, 361.1974.

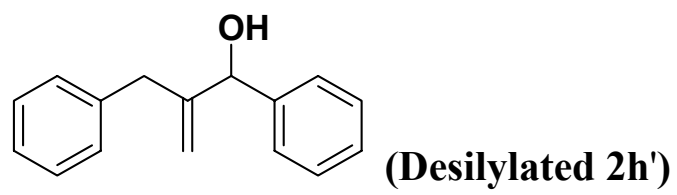

${ }^{1} \mathrm{H}$ NMR (400 MHz, $\left.\mathrm{CDCl}_{3}, \delta\right): 7.39$ (m, 4H), 7.29-7.35 (m, 3H), 7.22-7.24 (m, 1H), 7.13-7.15 (m, 2H), $5.37(\mathrm{~s}, 1 \mathrm{H}) ; 5.15(\mathrm{~s}, 1 \mathrm{H}) ; 4.93(\mathrm{~s}, 1 \mathrm{H}) ; 3.38(\mathrm{~d}, J=15.5 \mathrm{~Hz}, 1 \mathrm{H}) ; 3.13$ (d, $J=15.5 \mathrm{~Hz}$, $1 \mathrm{H}) ; 1.24$ (brs, 1H).

${ }^{13} \mathrm{C}$ NMR (100 MHz, $\left.\mathrm{CDCl}_{3}, \delta\right): 150.6,142.0,139.3,129.4,128.7,128.5,128.1,127.0,126.4$, 112.4, 76.7, 39.2.

IR (NaCl, thin film): 3377, 3061, 3028, 2919, 1494, 1453, 1025, 909, 750, 699.

HRMS-ESI (m / z): [M + Na] $]^{+}$calcd for $\mathrm{C}_{16} \mathrm{H}_{16} \mathrm{ONa}$, 247.1099; found, 247.1101. 


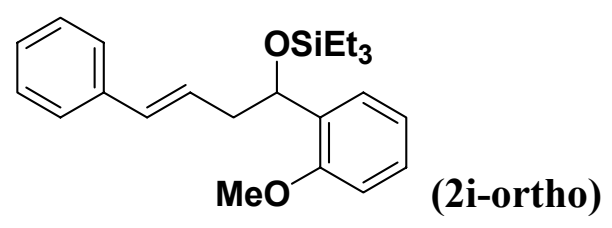

The reaction of allylbenzene and $o$-anisaldehyde $(60 \mu \mathrm{L}, 0.5 \mathrm{mmol})$ with $\mathrm{Ni}(\operatorname{cod})_{2}, \mathrm{Ph}_{3} \mathrm{P}(52 \mathrm{mg}$, $0.2 \mathrm{mmol}, 40 \mathrm{~mol} \%)$ and TESOTf ( $197 \mu \mathrm{L}, 0.875 \mathrm{mmol})$, triethylamine in toluene following the general procedure 3 above afforded 2i-ortho and 2i'-ortho in $78 \%$ total yield according to ${ }^{1} \mathrm{H}$ NMR of the crude mixture and the ratio of 2i-ortho:2i'-ortho is $92: 8$. The $E / Z$ ratio of 2i-ortho is $>95: 5$. 2i-ortho was subjected to TBAF and the free alcohol was isolated as a colorless oil. Allylic alcohol 2i'-ortho was not isolated.

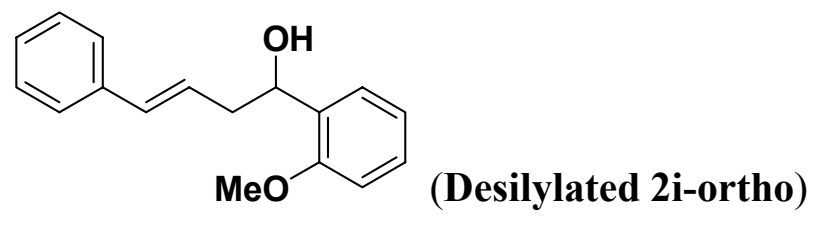

${ }^{1} \mathrm{H}$ NMR (400 MHz, $\left.\mathrm{CDCl}_{3}, \delta\right): 7.22-7.43(\mathrm{~m}, 7 \mathrm{H}) ; 7.02(\mathrm{t}, J=7.5 \mathrm{~Hz}, 1 \mathrm{H}) ; 6.93(\mathrm{~d}, J=8.1 \mathrm{~Hz}$, $1 \mathrm{H}) ; 6,52(\mathrm{~d}, J=15.9 \mathrm{~Hz}, 1 \mathrm{H}) ; 6.31(\mathrm{dt}, J=7.2,15.9 \mathrm{~Hz}, 1 \mathrm{H}) ; 5.09$ (dd, $J=5.1,7.5 \mathrm{~Hz}, 1 \mathrm{H})$; 3.89 (s, 3H); 2.69-2.81 (m, 3H).

${ }^{13} \mathrm{C}$ NMR $\left(100 \mathrm{MHz}, \mathrm{CDCl}_{3}, \delta\right): 156.5,137.6,132.8,131.9,128.6,128.5,127.3,127.0,126.9$, $126.3,120.9,110.6,70.2,55.5,41.3$.

IR (NaCl, thin film): 3399, 3026, 2935, 2836, 1601, 1491, 1464, 1438, 1287, 1240, 1181, 1049, 1029, 966, 753, 694.

HRMS-ESI (m/z): [M+Na] $]^{+}$calcd for $\mathrm{C}_{23} \mathrm{H}_{32} \mathrm{O}_{2} \mathrm{SiNa}, 391.2069$; found, 391.2053. 


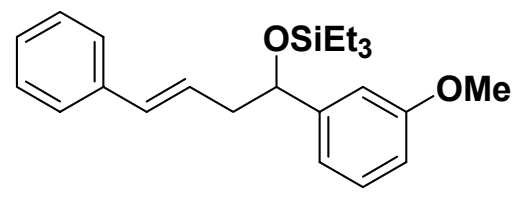

\section{(2i-meta)}

The reaction of allylbenzene and $m$-anisaldehyde $(61 \mu \mathrm{L}, 0.5 \mathrm{mmol})$ with $\mathrm{Ni}(\operatorname{cod})_{2}, \mathrm{Ph}_{3} \mathrm{P}(52 \mathrm{mg}$, $0.2 \mathrm{mmol}, 40 \mathrm{~mol} \%)$ and TESOTf ( $197 \mu \mathrm{L}, 0.875 \mathrm{mmol})$, triethylamine in toluene following the general procedure 3 above afforded $2 \mathbf{i}$-meta and $2 \mathbf{i}$-meta in $96 \%$ total yield according to ${ }^{1} \mathrm{H}$ NMR of the crude mixture and the ratio of 2i-meta:2i'-meta is $92: 8$. The $E$ / $Z$ ratio of 2i-meta is $>95: 5$. Purification via flash chromatography on silica afforded 2i-meta as a colorless oil. Allylic alcohol 2i'-meta was not isolated.

${ }^{1} \mathrm{H}$ NMR (400 MHz, $\left.\mathrm{CDCl}_{3}, \delta\right): 7.25-7.41(\mathrm{~m}, 6 \mathrm{H}), 7.03(\mathrm{~m}, 1 \mathrm{H}), 7.0(\mathrm{~d}, J=7.6 \mathrm{~Hz}, 1 \mathrm{H}) ; 6.87$ (dd, $J=0.8,2.7 \mathrm{~Hz}, 1 \mathrm{H}) ; 6.48(\mathrm{~d}, J=15.9 \mathrm{~Hz}, 1 \mathrm{H}) ; 6.30(\mathrm{dt}, J=7.2,15.9 \mathrm{~Hz}, 1 \mathrm{H}) ; 3.87$ (s, $3 \mathrm{H})$; 2.61-2.75 (m, 2H), 0.99 (t, $J=7.9 \mathrm{~Hz}, 9 \mathrm{H}) ; 0.64$ (q, $J=7.9 \mathrm{~Hz}, 6 \mathrm{H})$.

${ }^{13} \mathrm{C}$ NMR (100 MHz, $\left.\mathrm{CDCl}_{3}, \delta\right): 159.7,147.1,137.9,134.0,133.8,132.3,129.2,128.9,128.7$, 128.6, 127.2, 127.1, 126.2, 118.4, 112.8, 111.3, 75.2, 55.3, 44.8, 7.0, 5.0.

IR ( $\mathrm{NaCl}$, thin film): 3027, 2954, 2910, 2876, 2835, 1601, 1587, 1488, 1456, 1435, 1359, 1320, 1284, 1263, 1153, 1083, 1050, 1006, 966, 943, 825, 779, 743, 699.

HRMS-ESI (m/z): [M+Na] calcd for $\mathrm{C}_{23} \mathrm{H}_{32} \mathrm{O}_{2} \mathrm{SiNa}, 391.2069$; found, 391.1750. 


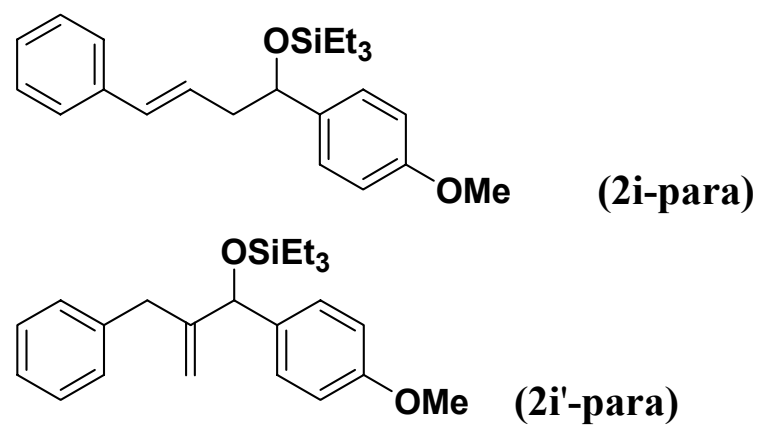

The reaction of allylbenzene and $o$-anisaldehyde $(61 \mu \mathrm{L}, 0.5 \mathrm{mmol})$ with $\mathrm{Ni}(\operatorname{cod})_{2}, \mathrm{Ph}_{3} \mathrm{P}(52 \mathrm{mg}$, $0.2 \mathrm{mmol}, 40 \mathrm{~mol} \%)$ and TESOTf ( $197 \mu \mathrm{L}, 0.875 \mathrm{mmol})$, triethylamine in toluene following the general procedure 3 above afforded 2i-para and 2i'-para in $99 \%$ total yield according to ${ }^{1} \mathrm{H}$ NMR of the crude mixture and the ratio of 2i-para:2i'-para is $92: 8$. The $E / Z$ ratio of 2i-para is $>95: 5$. Purification via flash chromatography on silica afforded 2i-para as a colorless oil. 2i'-para was not isolated.

In another experiment, general procedure 3 was followed, except that the reaction was carried out in five fold larger scale. The reaction was heated at $35^{\circ} \mathrm{C}$ and $9 \mathrm{~mL}$ toluene was used as the solvent. This reaction afforded 2i-para and 2i'-para in 98\% total yield according to ${ }^{1} \mathrm{H}$ NMR of the crude mixture and the ratio of 2i-para:2i'-para is $92: 8$. The $E / Z$ ratio of 2i-para is $>95: 5$. Purification via flash chromatography on silica afforded 2i-para as a colorless oil. 2i'-para was not isolated.

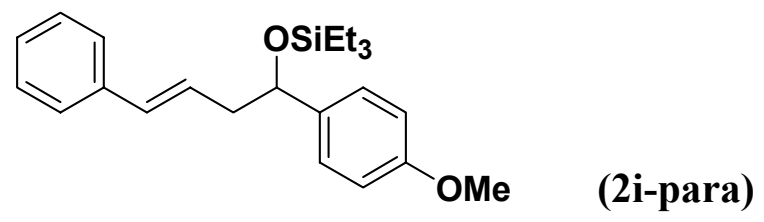

${ }^{1} \mathrm{H}$ NMR (400 MHz, $\left.\mathrm{CDCl}_{3}, \delta\right): 7.49$ (m, 7H); $7.00(\mathrm{~d}, J=8.6 \mathrm{~Hz}, 2 \mathrm{H}) ; 6.52(\mathrm{~d}, J=15.9 \mathrm{~Hz}, 1 \mathrm{H})$; 6.35 (dt, $J=7.2,15.9 \mathrm{~Hz}, 1 \mathrm{H}) ; 4.85$ (dd, $J=6.4,6.4 \mathrm{~Hz}, 1 \mathrm{H}) ; 3.89$ (s, 3H); 2.63-2.81 (m, 2H); 1.04 (t, $J=7.8 \mathrm{~Hz}, 9 \mathrm{H}) ; 0.70$ (q, $J=7.8 \mathrm{~Hz}, 6 \mathrm{H})$.

${ }^{13} \mathrm{C}$ NMR (100 MHz, $\left.\mathrm{CDCl}_{3}, \delta\right): 159.0,138.1,137.7,132.3,128.7,127.5,127.3$ 127.2, 126.3, 113.7, 75.0, 55.4, 45.1, 7.1, 5.2.

IR ( $\mathrm{NaCl}$, thin film): 3027, 2954, 2910, 2875, 1612, 1511, 1414, 1302, 1248, 1171, 1081, 1005 , 966, 836, 743, 693.

HRMS-ESI (m / z): [M + Na] $]^{+}$calcd for $\mathrm{C}_{23} \mathrm{H}_{32} \mathrm{O}_{2} \mathrm{SiNa}$, 391.2069; found, 391.2057. 
<smiles>CCOC(C/C=C/c1ccccc1)c1ccc2ccccc2c1</smiles><smiles>C=C(Cc1ccccc1)C(OCC)c1ccc2ccccc2c1</smiles>

$\left(2 \mathbf{j}^{\prime}\right)$

The reaction of allylbenzene and naphthaldehyde $(78 \mathrm{mg}, 0.5 \mathrm{mmol})$ with $\mathrm{Ni}(\mathrm{cod})_{2}, \mathrm{Ph}_{3} \mathrm{P}(52 \mathrm{mg}$, $0.2 \mathrm{mmol}, 40 \mathrm{~mol} \%)$ and TESOTf $(197 \mu \mathrm{L}, 0.875 \mathrm{mmol})$, triethylamine in toluene following the general procedure 3 above afforded $\mathbf{2} \mathbf{j}$ and $\mathbf{2} \mathbf{j}$ ' in $88 \%$ total yield according to ${ }^{1} \mathrm{H}$ NMR of the crude mixture and the ratio of $\mathbf{2} \mathbf{j}: \mathbf{2} \mathbf{j}$ is $95: 5$. The $E / Z$ ratio of $\mathbf{2} \mathbf{j}$ is $>95: 5$. Purification via flash chromatography on silica afforded $\mathbf{2} \mathbf{j}$ as a colorless oil. $\mathbf{2} \mathbf{j}$ ' was subjected to TBAF and the free alcohol was isolated by flash chromatography on silica as a colorless oil.<smiles>CCOC(C/C=C/c1ccccc1)c1ccc2ccccc2c1</smiles>

${ }^{1} \mathrm{H}$ NMR (400 MHz, $\left.\mathrm{CDCl}_{3}, \delta\right): 7.90-7.96(\mathrm{~m}, 4 \mathrm{H}) ; 7.67$ (d, $\left.J=1.6 \mathrm{~Hz}, 1 \mathrm{H}\right) ; 7.60-7.65$ (m, 2H); 7.30-7.59 (m, 5H); $6.54(\mathrm{~d}, J=15.9 \mathrm{~Hz}, 1 \mathrm{H}) ; 6.36(\mathrm{dt}, J=7.2,15.9 \mathrm{~Hz}, 1 \mathrm{H}) ; 5.05(\mathrm{dd}, J=5.4$, $7.2 \mathrm{~Hz}, 1 \mathrm{H}) ; 2.74-2.89(\mathrm{~m}, 2 \mathrm{H}) ; 1.03$ (t, $J=8.0 \mathrm{~Hz}, 9 \mathrm{H}) ; 0.70(\mathrm{dq}, J=2.9,8.0 \mathrm{~Hz}, 6 \mathrm{H})$.

${ }^{13} \mathrm{C}$ NMR (100 MHz, $\left.\mathrm{CDCl}_{3}, \delta\right): 142.9,137.9,133.4,133.1,132.4,128.7,128.1,128.1,127.9$, $127.1,126.2,126.1,125.7,124.6,75.5,44.9,7.0,5.1$.

IR ( $\mathrm{NaCl}$, thin film): 3026, 2954, 2910, 2875, 1507, 1496, 1457, 1239, 1123, 1083, 1005, 965 , $819,744$.

HRMS-ESI (m / z): [M + Na] $]^{+}$calcd for $\mathrm{C}_{26} \mathrm{H}_{32} \mathrm{OSiNa}$, 411.2120; found, 411.2167.<smiles>C=C(Cc1ccccc1)C(O)c1ccc2ccccc2c1</smiles>

\section{(Desilylated 2j')}

${ }^{1} \mathrm{H}$ NMR (400 MHz, $\left.\mathrm{CDCl}_{3}, \delta\right): 7.86-7.88$ (m, 4H); 7.48-7.55 (m, 3H); 7.20-7.36 (m, 3H); 7.13-7.16 (m, 2H); $5.43(\mathrm{~s}, 1 \mathrm{H}) ; 5.32(\mathrm{~s}, 1 \mathrm{H}) ; 4.97(\mathrm{~s}, 1 \mathrm{H}) ; 3.41(\mathrm{~d}, J=15.6 \mathrm{~Hz}, 1 \mathrm{H}), 3.16(\mathrm{~d}, J=$ $15.6 \mathrm{~Hz}, 1 \mathrm{H}), 2.02$ (brs, $1 \mathrm{H})$.

${ }^{13} \mathrm{C}$ NMR (100 MHz, $\left.\mathrm{CDCl}_{3}, \delta\right): 150.5,149.2,139.4,139.3,133.4,133.3,129.4,128.6,128.2$, $127.9,126.4,126.4,126.2,126.0,124.9,112.8,77.4,39.2$.

IR ( $\mathrm{NaCl}$, thin film): 3365, 3058, 2923, 1495, 1453, 1031, 908, 819, 745, 700. HRMS-ESI (m / z): [M + Na $]^{+}$calcd for $\mathrm{C}_{20} \mathrm{H}_{18} \mathrm{ONa}$, 297.1255; found, 297.1260. 


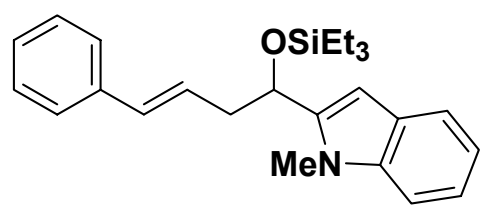

(2k)<smiles>C=C(Cc1ccccc1)C(OCC)c1cc2ccccc2n1C</smiles>

$\left(2 k^{\prime}\right)$

The reaction of allylbenzene and 1-methyl-2-indolecarboxaldehyde (79.6 $\mathrm{mg}, 0.5 \mathrm{mmol})$ with $\mathrm{Ni}(\text { cod })_{2}, \mathrm{Ph}_{3} \mathrm{P}(52 \mathrm{mg}, 0.2 \mathrm{mmol}, 40 \mathrm{~mol} \%)$ and TESOTf $(197 \mu \mathrm{L}, 0.875 \mathrm{mmol})$, triethylamine in toluene following the general procedure 3 above afforded $\mathbf{2} \mathbf{k}$ in $57 \%$ total yield according to ${ }^{1} \mathrm{H}$ NMR of the crude mixture and the ratio of $\mathbf{2} \mathbf{k}: \mathbf{2} \mathbf{k}^{\prime}$ is $>95: 5$. The $E / Z$ ratio of $\mathbf{2 k}$ is $>95: 5 . \mathbf{2} \mathbf{k}^{\prime}$ was not detected. $\mathbf{2 k}$ was subjected to TBAF and the free alcohols were isolated by flash chromatography on silica (buffered with $\mathrm{Et}_{3} \mathrm{~N}$ ) as colorless oils.

In another experiment, the reaction of allylbenzene and 1-methyl-2-indolecarboxaldehyde (79.6 $\mathrm{mg}, 0.5 \mathrm{mmol}$ ) with $\mathrm{Ni}(\mathrm{cod})_{2}, \mathrm{Cy}_{2} \mathrm{PhP}(56 \mathrm{mg}, 0.2 \mathrm{mmol}, 40 \mathrm{~mol} \%)$ and TESOTf (197 $\mu \mathrm{L}, 0.875$ mmol), triethylamine in toluene following the general procedure 3 above afforded $\mathbf{2} \mathbf{k}^{\prime}$ and $\mathbf{2} \mathbf{k}$ in $56 \%$ total yield according to ${ }^{1} \mathrm{H}$ NMR of the crude mixture and the ratio of $\mathbf{2} \mathbf{k}^{\prime}: \mathbf{2} \mathbf{k}$ is $80: 20$. The $E / Z$ ratio of $\mathbf{2 k}$ is $>95: 5$. Both $\mathbf{2} \mathbf{k}^{\prime}$ and $\mathbf{2} \mathbf{k}$ were subjected to TBAF and the free alcohols were isolated by flash chromatography on silica (buffered with $\mathrm{Et}_{3} \mathrm{~N}$ ) as colorless oils.

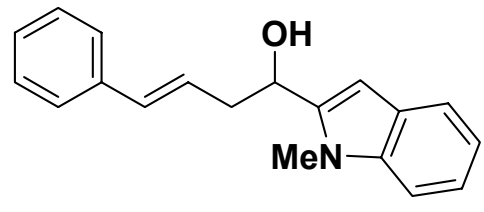

\section{(Desilylated 2k)}

${ }^{1} \mathrm{H}$ NMR (400 MHz, $\left.\mathrm{CDCl}_{3}, \delta\right): 7.63(\mathrm{~d}, J=7.8 \mathrm{~Hz}, 1 \mathrm{H}) ; 7.20-7.41(\mathrm{~m}, 7 \mathrm{H}) ; 7.14(\mathrm{t}, J=7.8 \mathrm{~Hz}$, $1 \mathrm{H}) ; 6.62(\mathrm{~d}, J=15.8 \mathrm{~Hz}, 1 \mathrm{H}) ; 6.55(\mathrm{~s}, 1 \mathrm{H}) ; 6.34(\mathrm{dt}, J=7.3,15.8 \mathrm{~Hz}, 1 \mathrm{H}) ; 5.01(\mathrm{~m}, 1 \mathrm{H}) ; 3.86$ (s, 3H); 2.93-2.99 (m, 2H); 1.93 (brs, 1H).

${ }^{13} \mathrm{C}$ NMR (100 MHz, $\left.\mathrm{CDCl}_{3}, \delta\right): 149.2,141.3,138.1,137.2,133.8,128.7,127.6,126.4,125.7$, $122.1,121.0,119.8,109.3,99.4,66.9,40.2,30.4$.

IR (NaCl, thin film): 3640, 3026, 2953, 2910, 2875, 1467, 1339, 1237, 1073, 1006, 966, 744.

HRMS-ESI (m / z): [M + Na $]^{+}$calcd for $\mathrm{C}_{19} \mathrm{H}_{19} \mathrm{ONNa}$, 300.1364; found, 300.1365. 


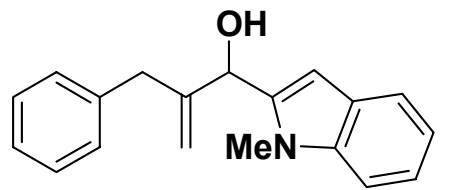

\section{(Desilylated 2k')}

${ }^{1} \mathrm{H}$ NMR (400 MHz, $\left.\mathrm{CDCl}_{3}, \delta\right): 7.63$ (d, 1H); 7.12-7.38 (m, 8H); 6.49 (s, 1H); 5.38 (s, 1H); 5.31 (s, $1 \mathrm{H}) ; 5.14(\mathrm{~s}, 1 \mathrm{H}) ; 3.70(\mathrm{~s}, 3 \mathrm{H}) ; 3.54(\mathrm{~d}, J=15.3 \mathrm{~Hz}, 1 \mathrm{H}) ; 3.33$ (d, $J=15.3 \mathrm{~Hz}, 1 \mathrm{H}) ; 1.98$ (d, $J$ $=5.1 \mathrm{~Hz}, 1 \mathrm{H})$.

${ }^{13} \mathrm{C}$ NMR (100 MHz, $\left.\mathrm{CDCl}_{3}, \delta\right): 148.7,139.6,139.1,138.4,129.3,128.6,127.3,126.6$, 122.0, 121.0, 119.7, 113.2, 109.3, 101.5, 69.6, 40.2, 30.3 .

IR ( $\mathrm{NaCl}$, thin film): 3349, 3059, 3027, 2923, 1649, 1601, 1494, 1468, 1453, 1318, 1234, 1030, 968, 907, 751, 737, 700 .

HRMS-ESI (m / z): [M + Na $]^{+}$calcd for $\mathrm{C}_{19} \mathrm{H}_{19} \mathrm{NONa}, 300.1364$; found, 300.1369 . 


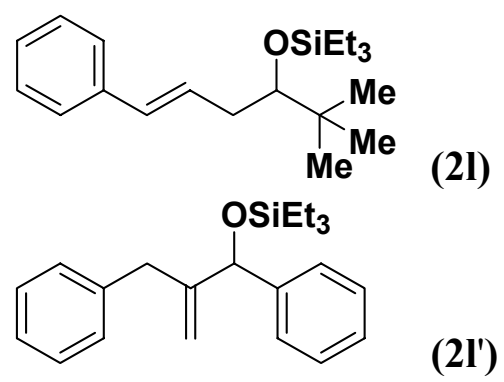

The reaction of allylbenzene and pivaldehyde $(55 \mu \mathrm{L}, 0.5 \mathrm{mmol})$ with $\mathrm{Ni}(\operatorname{cod})_{2}, \mathrm{Ph}_{3} \mathrm{P}(52 \mathrm{mg}, 0.2$ mmol, $40 \mathrm{~mol} \%)$ and TESOTf $(197 \mu \mathrm{L}, 0.875 \mathrm{mmol})$, triethylamine in toluene following the general procedure 3 above afforded $2 \mathrm{l}$ in $65 \%$ total yield according to ${ }^{1} \mathrm{H}$ NMR of the crude mixture and the ratio of 21:2l' is $>95: 5$. The $E / Z$ ratio of $\mathbf{2 l}$ is 78:22. 2l' was not detected. Purification via flash chromatography on silica afforded $\mathbf{2 l}$ as a colorless oil.

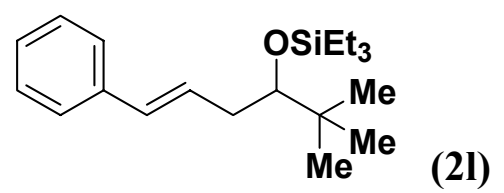

${ }^{1} \mathrm{H}$ NMR (400 MHz, $\left.\mathrm{CDCl}_{3}, \delta\right): 7.22-7.40(\mathrm{~m}, 5 \mathrm{H}) ; 6.43(\mathrm{~d}, J=15.9 \mathrm{~Hz}, 1 \mathrm{H}) ; 6.32(\mathrm{dt}, J=7.1$, $15.9 \mathrm{~Hz}, 1 \mathrm{H}) ; 3.50$ (dd, $J=3.4,7.7 \mathrm{~Hz}, 1 \mathrm{H}) ; 2.49-2.55(\mathrm{~m}, 1 \mathrm{H}), 2.28-2.35(\mathrm{~m}, 1 \mathrm{H}), 1.00(\mathrm{t}, J=$ $8.0 \mathrm{~Hz}, 9 \mathrm{H}) ; 0.96$ (s, 9H); 0.64 (q, $J=8.0 \mathrm{~Hz}, 6 \mathrm{H})$.

${ }^{13} \mathrm{C}$ NMR (100 MHz, $\left.\mathrm{CDCl}_{3}, \delta\right): 138.1,131.3,129.7,128.7,127.0,126.1,81.0,37.4,36.2,26.6$, 7.3, 5.7.

HRMS-ESI (m/z): $[\mathrm{M}+\mathrm{Na}]^{+}$calcd for $\mathrm{C}_{20} \mathrm{H}_{34} \mathrm{OSiNa}, 341.2277$; found, 341.2263 . 


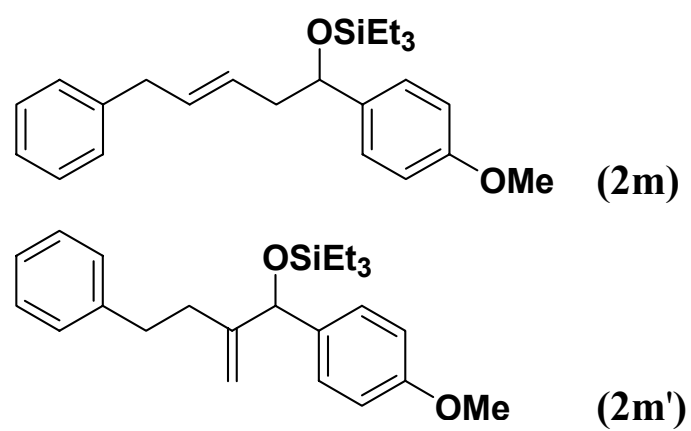

The reaction of 4-phenyl-1-butene and $o$-anisaldehyde $(61 \mu \mathrm{L}, 0.5 \mathrm{mmol})$ with $\mathrm{Ni}(\operatorname{cod})_{2}, \mathrm{Ph}_{3} \mathrm{P}$ (52 $\mathrm{mg}, 0.2 \mathrm{mmol}, 40 \mathrm{~mol} \%)$ and TESOTf $(197 \mu \mathrm{L}, 0.875 \mathrm{mmol})$, triethylamine in toluene following the general procedure 3 above afforded $\mathbf{2} \mathbf{m}$ and $\mathbf{2} \mathbf{m}$ ' in $91 \%$ total yield according to ${ }^{1} \mathrm{H}$ NMR of the crude mixture and the ratio of $\mathbf{2 m : 2 m}$ ' is $92: 8$. The $E / Z$ ratio of $\mathbf{2 m}$ is $68: 32$. Purification via flash chromatography on silica afforded $\mathbf{2} \mathbf{m}$ and $\mathbf{2} \mathbf{m}$ ' as colorless oils.

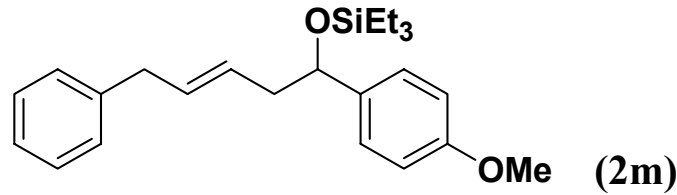

${ }^{1} \mathrm{H}$ NMR (400 MHz, $\left.\mathrm{CDCl}_{3}, \delta\right): 7.13-7.42(\mathrm{~m}, 7 \mathrm{H}), 6.92(\mathrm{~d}, J=8.7 \mathrm{~Hz}, 2 \mathrm{H}), 5.49-5.69(\mathrm{~m}, 2 \mathrm{H})$, $4.76(\mathrm{t}, J=6.3 \mathrm{~Hz}, 0.33 \mathrm{H}), 4.70(\mathrm{t}, J=6.4 \mathrm{~Hz}, 0.67 \mathrm{H}), 3.87(\mathrm{~s}, 3 \mathrm{H}), 3.37-3.39(\mathrm{~m}, 2 \mathrm{H})$, $2.35-2.81(\mathrm{~m}, 2 \mathrm{H}), 0.96$ (t, $J=7.9 \mathrm{~Hz}, 6 \mathrm{H}) ; 0.60$ (q, $J=7.9 \mathrm{~Hz}, 9 \mathrm{H})$.

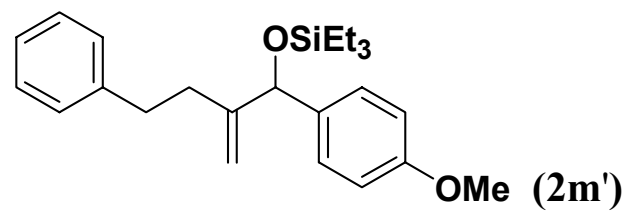

${ }^{1} \mathrm{H}$ NMR (400 MHz, $\mathrm{CDCl}_{3}, \delta$ ): 7.13-7.42 (m, 7H), 6.93 (d, $\left.J=8.7 \mathrm{~Hz}, 2 \mathrm{H}\right), 5.34(\mathrm{~s}, 1 \mathrm{H}), 5.19$ (s, $1 \mathrm{H}), 5.00(\mathrm{~s}, 1 \mathrm{H}), 3.86(\mathrm{~s}, 3 \mathrm{H}), 2.61-2.79(\mathrm{~m}, 2 \mathrm{H}), 2.26-2.42(\mathrm{~m}, 1 \mathrm{H}), 2.16-2.22(\mathrm{~m}, 1 \mathrm{H}), 1.07$ (t, $J=7.8 \mathrm{~Hz}, 9 \mathrm{H}) ; 0.74$ (q, $J=7.9 \mathrm{~Hz}, 6 \mathrm{H})$. 
<smiles>CCOC(CC=CC(C)C)c1ccccc1</smiles><smiles>C=C(CC(C)C)C(OCC)c1ccccc1</smiles>

\section{$\left(2 n^{\prime}\right)$}

The reaction of 4-methyl-1-pentene and $o$-anisaldehyde $(61 \mu \mathrm{L}, 0.5 \mathrm{mmol})$ with $\mathrm{Ni}(\operatorname{cod})_{2}$, EtOPh $_{2} \mathrm{P}$ (43 $\left.\mu \mathrm{l}, 0.2 \mathrm{mmol}, 40 \mathrm{~mol} \%\right)$ and TESOTf $(197 \mu \mathrm{L}, 0.875 \mathrm{mmol})$, triethylamine in toluene following the general procedure 3 above afforded $\mathbf{2 n}$ and $\mathbf{2 n}$ ' in $82 \%$ total yield according to ${ }^{1} \mathrm{H}$ NMR of the crude mixture and the ratio of $\mathbf{2 n}: \mathbf{2 n}$ ' is $>95: 5$. The $E$ / $Z$ ratio of $\mathbf{2 n}$ is 81:19. 2n' was not detected. Purification via flash chromatography on silica afforded $\mathbf{2 n}$ as a colorless oil.

In another experiment, a $10 \mathrm{~mL}$ round bottom flask and a stir bar were oven-dried and brought into a glove box. $\mathrm{Ni}(\mathrm{cod})_{2}(27.5 \mathrm{mg}, 0.2 \mathrm{mmol}, 20 \mathrm{~mol} \%)$ and dicyclohexylphenylphosphine (55 $\mathrm{mg}, 0.4 \mathrm{mmol}, 40 \mathrm{~mol} \%$ ) were added to the round bottom flask, the flask was sealed with a septum, and the sealed flask was brought out of the glove box and connected to an argon line. The catalyst mixture was dissolved in toluene $(2.5 \mathrm{~mL})$ under argon and stirred 5 min at room temperature. 4-methyl-1-pentene $(633 \mu \mathrm{L}, 5 \mathrm{mmol}, 1000 \mathrm{~mol} \%)$ was added. Triethylamine (418 $\mu \mathrm{L}, 3 \mathrm{mmol}, 600 \mathrm{~mol} \%)$ was added. Benzaldehyde (51 $\mu \mathrm{L}, 0.5 \mathrm{mmol}, 100 \mathrm{~mol} \%)$ was added to the reaction mixture, followed by TESOTf $(197 \mu \mathrm{L}, 0.875 \mathrm{mmol}, 175 \mathrm{~mol} \%)$. The mixture was stirred at room temperature for $14 \mathrm{~h}$. The mixture was filtered through a plug of silica gel. Solvent was removed under reduced pressure and NMR of the crude mixture indicated the ratio of $2 \mathbf{n}^{\prime}: \mathbf{2 n}$ is $75: 25$. Purification via flash chromatography on silica afforded $\mathbf{2 n}$ ' in $44 \%$ isolated yield as a colorless oil and $\mathbf{2 n}$ in $10 \%$ isolated yield.

This reaction can be run according to general procedure 3, which also afforded $\mathbf{2 n}$ ' and $\mathbf{2 n}$ in similar yield. 
<smiles>CCOC(CC=CC(C)C)c1ccccc1</smiles>

(2n)

${ }^{1} \mathrm{H}$ NMR (400 MHz, $\left.\mathrm{CDCl}_{3}, \delta\right): 7.30(\mathrm{~m}, 5 \mathrm{H}) ; 5.40(\mathrm{~m}, 2 \mathrm{H}) ; 4.63(\mathrm{dd}, J=5.3,7.3 \mathrm{~Hz}, 1 \mathrm{H}) ; 2.41$ (quintet, $J=5.3 \mathrm{~Hz}, 1 \mathrm{H}$ ); 2.30 (quintet, $J=5.5 \mathrm{~Hz}, 1 \mathrm{H}$ ); 2.24 (septet, $J=6.7 \mathrm{~Hz}, 1 \mathrm{H}$ ); 2.00 (m, $2 \mathrm{H}) ; 0.95$ (dd, $J=6.7,7.6 \mathrm{~Hz}, 6 \mathrm{H}) ; 0.89(\mathrm{t}, J=7.9 \mathrm{~Hz}, 9 \mathrm{H}) ; 0.62(\mathrm{q}, J=7.9 \mathrm{~Hz}, 6 \mathrm{H})$.

${ }^{13} \mathrm{C}$ NMR (100 MHz, $\left.\mathrm{CDCl}_{3}, \delta\right): 145.6,140.2,128.1,127.0,126.1,123.7,75.7,44.5,31.3,22.6$, 7.01, 5.0.

HRMS-ESI (m / z): [M + Na] $]^{+}$calcd for $\mathrm{C}_{19} \mathrm{H}_{32} \mathrm{OSiNa}$, 327.212; found, 327.212.

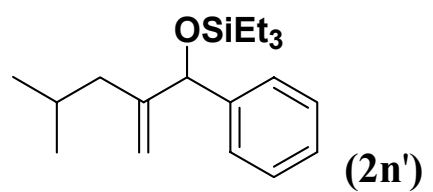

${ }^{1} \mathrm{H}$ NMR (400 MHz, $\left.\mathrm{CDCl}_{3}, \delta\right): 7.36(\mathrm{~d}, J=7.8 \mathrm{~Hz}, 2 \mathrm{H}) ; 7.32(\mathrm{t}, J=7.1 \mathrm{~Hz}, 2 \mathrm{H}) ; 7.25(\mathrm{t}, J=7.1$, 1H); 5.30 (bs, 1H); 5.12 (bs, 1H); 4.87 (bs, 1H); $1.65-1.85$ (m, 3H); 0.93 (t, $J=8.0 \mathrm{~Hz}, 9 \mathrm{H})$; $0.84(\mathrm{~d}, J=6.4 \mathrm{~Hz}, 3 \mathrm{H}) ; 0.82(\mathrm{~d}, J=6.2 \mathrm{~Hz}, 3 \mathrm{H}) ; 0.60(\mathrm{dq}, J=1.3,8.3 \mathrm{~Hz}, 6 \mathrm{H})$.

${ }^{13} \mathrm{C}$ NMR (100 MHz, $\left.\mathrm{CDCl}_{3}, \delta\right): 150.5,143.7,128.1,127.1,126.7,110.7,77.9,41.1,26.3,23.0$, 22.6, 7.0, 5.0.

IR (NaCl, thin film): 2955, 2877, 1646, 1454, 1088, 1067, 743.

HRMS-ESI (m / z): [M + Na] $]^{+}$calcd for $\mathrm{C}_{19} \mathrm{H}_{32} \mathrm{OSiNa}$, 327.211; found, 327.212. 

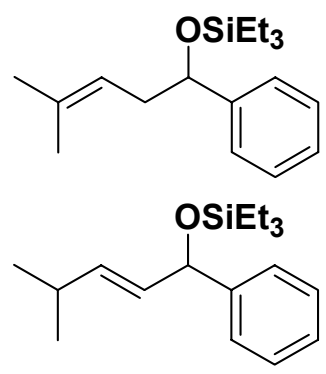

\section{Triethyl-(4-methyl-1-phenyl-pent-3-enyloxy)-silane (2o).}

Triethyl-(4-methyl-1-phenyl-pent-2-enyloxy)-silane (2o').

The reaction of 3-methyl-1-butene and benzaldehyde $(51 \mu \mathrm{L}, 0.5 \mathrm{mmol})$ with $\mathrm{Ni}(\mathrm{cod})_{2}, \mathrm{EtOPh}_{2} \mathrm{P}$ (43 $\mu \mathrm{l}, 0.2 \mathrm{mmol}, 40 \mathrm{~mol} \%$ ) and TESOTf $(197 \mu \mathrm{L}, 0.875 \mathrm{mmol})$, triethylamine in toluene following the general procedure 3 above afforded 20 and 20 ' in $95 \%$ total yield according to ${ }^{1} \mathrm{H}$ NMR of the crude mixture and the ratio of 20:20' is 86:14. The $E / Z$ ratio of $\mathbf{2 0}$ ' is $>95: 5$. Purification via flash chromatography on silica afforded 2o. 20' was not isolated.<smiles>CCOC(CC=C(C)C)c1ccccc1</smiles>

\section{(2o)}

${ }^{1} \mathrm{H}$ NMR (400 MHz, $\left.\mathrm{CDCl}_{3}, \delta\right): 7.27-7.43$ (m, 5H); 5.19-5.24 (m, 1H); 4.68 (dd, J= 5.8, 7.2 Hz, $1 \mathrm{H}) ; 2.36-2.54(\mathrm{~m}, 2 \mathrm{H}) ; 1.74(\mathrm{~d}, J=0.8 \mathrm{~Hz}, 3 \mathrm{H}) ; 1.58(\mathrm{~s}, 3 \mathrm{H}) ; 0.95$ (t, $J=7.8 \mathrm{~Hz}, 9 \mathrm{H}) ; 0.60(\mathrm{dq}$, $J=3.4,7.8 \mathrm{~Hz}, 6 \mathrm{H})$.

${ }^{13} \mathrm{C} \mathrm{NMR}\left(100 \mathrm{MHz}, \mathrm{CDCl}_{3}, \delta\right): 145.8,133.6,128.1,127.0,126.1,121.0,75.4,40.0,26.0,18.0$, 7.0, 5.0.

IR ( $\mathrm{NaCl}$, thin film): 3028, 2956, 2877, 2912, 1454, 1414, 1377, 1239, 1089, 1069, 1005, 941, $744,699$.

HRMS-ESI (m / z): [M + Na] $]^{+}$calcd for $\mathrm{C}_{18 \mathrm{H}} \mathrm{H}_{30 \mathrm{OSiNa}}$, 313.1964; found, 313.1966 . 
<smiles>CCOC(CC=C1CCCCC1)c1ccccc1</smiles><smiles>C=C(C1CCCCC1)C(OCC)c1ccccc1</smiles><smiles>CCOC(C=CC1CCCCC1)c1ccccc1</smiles>

(3p)

The reaction of vinylcyclohexane and benzaldehyde $(51 \mu \mathrm{L}, 0.5 \mathrm{mmol})$ with $\mathrm{Ni}(\mathrm{cod})_{2}, \mathrm{EtOPh}_{2} \mathrm{P}$ (43 $\mu \mathrm{l}, 0.2 \mathrm{mmol}, 40 \mathrm{~mol} \%)$ and TESOTf $(197 \mu \mathrm{L}, 0.875 \mathrm{mmol})$, triethylamine in toluene following the general procedure 3 above afforded $\mathbf{2 p}$ and $\mathbf{3 p}$ in $99 \%$ total yield according to ${ }^{1} \mathrm{H}$ NMR of the crude mixture and the ratio of $\mathbf{2 p : 3 p}$ is $75: 25$. The $E / Z$ ratio of $\mathbf{3 p}$ is $>95: 5$. Purification via flash chromatography on silica afforded a mixture of $\mathbf{2 p}$ and $\mathbf{3 p}$.

In another experiment, a $10 \mathrm{~mL}$ round bottom flask and a stir bar were oven-dried and brought into a glove box. $\mathrm{Ni}(\operatorname{cod})_{2}(27.5 \mathrm{mg}, 0.2 \mathrm{mmol}, 20 \mathrm{~mol} \%)$ and dicyclohexylphenylphosphine (55 mg, $0.4 \mathrm{mmol}, 40 \mathrm{~mol} \%$ ) were added to the round bottom flask, the flask was sealed with a septum, and the sealed flask was brought out of the glove box and connected to an argon line. The catalyst mixture was dissolved in toluene $(2.5 \mathrm{~mL})$ under argon and stirred 5 min at room temperature. Vinylcyclohexane $(856 \mu \mathrm{L}, 6.25 \mathrm{mmol}, 1250 \mathrm{~mol} \%)$ was added. Triethylamine (418 $\mu \mathrm{L}, 3 \mathrm{mmol}, 600 \mathrm{~mol} \%)$ was added. Benzaldehyde (51 $\mu \mathrm{L}, 0.5 \mathrm{mmol}, 100 \mathrm{~mol} \%$ ) was added, followed by TESOTf $(197 \mu \mathrm{L}, 0.875 \mathrm{mmol}, 175 \mathrm{~mol} \%)$. The mixture was stirred at room temperature for $16 \mathrm{~h}$. The mixture was filtered through a plug of silica gel. 1H NMR of the crude mixture indicated that $\mathbf{2} \mathbf{p}^{\prime}$ is the minor product, along with homoallylic product $\mathbf{2 p}$ and 1,3-disubstituted allylic product $\mathbf{3 p}$ as major products. Purification via flash chromatography on silica afforded $\mathbf{2} \mathbf{p}$ ' in $5 \%$ isolated yield as a colorless oil. 
<smiles>CCOC(CC=C1CCCCC1)c1ccccc1</smiles>

(2p)

${ }^{1} \mathrm{H}$ NMR (400 MHz, $\left.\mathrm{CDCl}_{3}, \delta\right): 7.24-7.42(\mathrm{~m}, 5 \mathrm{H}) ; 5.14(\mathrm{t}, J=7.4 \mathrm{~Hz}, 1 \mathrm{H}) ; 4.66(\mathrm{t}, J=6.4 \mathrm{~Hz}$, $1 \mathrm{H}) ; 2.37-2.52(\mathrm{~m}, 2 \mathrm{H}) ; 2.00-2.11(\mathrm{~m}, 3 \mathrm{H}) ; 1.50-1.78(\mathrm{~m}, 3 \mathrm{H}) ; 1.03-1.48(\mathrm{~m}, 4 \mathrm{H}) ; 0.94(\mathrm{t}, J=$ $7.9 \mathrm{~Hz}, 9 \mathrm{H}) ; 0.59(\mathrm{dq}, J=2.8,7.9 \mathrm{~Hz}, 6 \mathrm{H})$.

${ }^{13} \mathrm{C} \mathrm{NMR}\left(100 \mathrm{MHz}, \mathrm{CDCl}_{3}, \delta\right): 145.7,141.6,128.0,127.0,126.2,117.5,75.6,39.0,37.5,29.0$, 28.7, 27.8, 27.1, 7.0, 5.0.

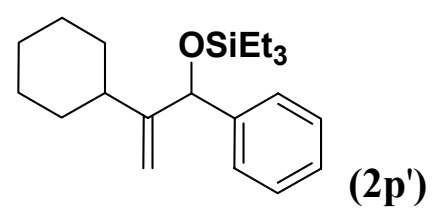

${ }^{1} \mathrm{H}$ NMR $\left(400 \mathrm{MHz}, \mathrm{CDCl}_{3}, \delta\right): 7.33(\mathrm{~d}, J=8.6 \mathrm{~Hz}, 2 \mathrm{H}) ; 7.29(\mathrm{t}, J=7.9,2 \mathrm{H}) ; 7.22(\mathrm{t}, J=7.0 \mathrm{~Hz}$, $1 \mathrm{H}) ; 5.23(\mathrm{dd}, J=1.3,1.3 \mathrm{~Hz}, 1 \mathrm{H}) ; 5.14(\mathrm{~s}, 1 \mathrm{H}) ; 4.90(\mathrm{~s}, 1 \mathrm{H}) ; 1.2-2.0(\mathrm{~m}, 11 \mathrm{H}) ; 0.91(\mathrm{t}, J=7.9$ $\mathrm{Hz}, 9 \mathrm{H}) ; 0.58(\mathrm{dq}, \mathrm{J}=0.5,7.8 \mathrm{~Hz}, 6 \mathrm{H})$.

${ }^{13} \mathrm{C} \mathrm{NMR}\left(125 \mathrm{MHz}, \mathrm{CDCl}_{3}, \delta\right): 157.7,143.7,128.0,127.1,126.9,108.2,77.6,39.5,34.5,33.5$, 27.1, 27.0, 26.5, 7.1, 5.0.

IR (NaCl, thin film): 2954, 2927, 2876, 1644, 1493, 1449, 1239, 1090, 858, 699.

HRMS-ESI (m / z): $[\mathrm{M}+\mathrm{Na}]^{+}$calcd for $\mathrm{C}_{21} \mathrm{H}_{34} \mathrm{OSiNa}$, 353.227; found, 353.227.<smiles>CCOC(C=CC1CCCCC1)c1ccccc1</smiles>

(3p)

${ }^{1} \mathrm{H}$ NMR (400 MHz, $\left.\mathrm{CDCl}_{3}, \delta\right): 7.24-7.42(\mathrm{~m}, 5 \mathrm{H}) ; 5.69(\mathrm{dd}, J=6.5,15.4 \mathrm{~Hz}, 1 \mathrm{H}) ; 5.56(\mathrm{dd}, J=$ 7.0, $15.4 \mathrm{~Hz}, 1 \mathrm{H}) ; 5.18(\mathrm{~d}, J=7.0 \mathrm{~Hz}, 1 \mathrm{H}) ; 2.00-2.11(\mathrm{~m}, 3 \mathrm{H}) ; 1.63-1.78(\mathrm{~m}, 1 \mathrm{H}) ; 1.50-1.78(\mathrm{~m}$, $3 \mathrm{H}) ; 1.03-1.48(\mathrm{~m}, 4 \mathrm{H}) ; 1.00$ (t, $J=8.0 \mathrm{~Hz}, 9 \mathrm{H}) ; 0.67$ (dq, $J=2.3,8.0 \mathrm{~Hz}, 6 \mathrm{H})$.

${ }^{13} \mathrm{C}$ NMR (100 MHz, $\left.\mathrm{CDCl}_{3}, \delta\right): 144.8,136.9,131.2,128.2,126.9,126.1,75.9,40.4,33.0,32.9$, 26.4, 26.2, 7.1, 5.2.

The following IR and HRMS data is from a mixture of $\mathbf{2 p}$ and $\mathbf{2} \mathbf{p}$ '.

IR ( $\mathrm{NaCl}$, thin film): 2954, 2928, 2876, 2853, 1449, 1414, 1238, 1086, 1067, 1007, 969, 829, $744,699$.

HRMS-ESI (m / z): $[\mathrm{M}+\mathrm{Na}]^{+}$calcd for $\mathrm{C}_{21} \mathrm{H}_{34} \mathrm{OSiNa}$, 353.2277; found, 353.2267. 


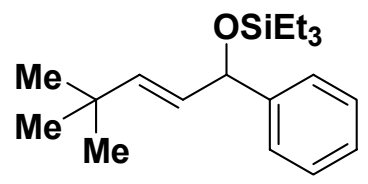

(3q)

The reaction of 3,3-dimethyl-1-butene and benzaldehyde $(51 \mu \mathrm{L}, 0.5 \mathrm{mmol})$ with $\mathrm{Ni}(\mathrm{cod})_{2}$, $\mathrm{EtOPh}_{2} \mathrm{P}(43 \mu \mathrm{l}, 0.2 \mathrm{mmol}, 40 \mathrm{~mol} \%)$ and TESOTf $(197 \mu \mathrm{L}, 0.875 \mathrm{mmol})$, triethylamine in toluene following the general procedure 3 above afforded $\mathbf{3 q}$ only in $14 \%$ total yield according to ${ }^{1} \mathrm{H}$ NMR of the crude mixture. Purification via flash chromatography on silica afforded $\mathbf{3 q}$ as a colorless oil.

${ }^{1} \mathrm{H}$ NMR $\left(400 \mathrm{MHz}, \mathrm{CDCl}_{3}, \delta\right): 7.30-7.41(\mathrm{~m}, 5 \mathrm{H}), 5.82(\mathrm{~d}, J=14.6 \mathrm{~Hz}, 1 \mathrm{H}), 5.59$ (dd, $J=14.6$, $7.0 \mathrm{~Hz}, 1 \mathrm{H}), 5.18$ (m, 1H), 1.88 (brs, $1 \mathrm{H}), 1.05$ (s, 9H). ${ }^{13} \mathrm{C} \mathrm{NMR}\left(100 \mathrm{MHz}, \mathrm{CDCl}_{3}, \delta\right.$ ): 149.2, $143.8,128.6,127.6,127.3,126.4,75.6,33.1,29.6$.

IR (NaCl, thin film): 3657, 2954, 2876, 1457, 1238, 966, 737, 691.

HRMS-ESI (m/z): [M+Na] $]^{+}$calcd for $\mathrm{C}_{19} \mathrm{H}_{32} \mathrm{OSiNa}, 327.2115$; found, 327.2105 . 
<smiles>C=C(C)C(OCC)c1ccc2ccccc2c1</smiles><smiles>C=CCC(OCC)c1ccc2ccccc2c1</smiles>

$\left(2 \mathbf{r}^{\prime}\right)$

(2r)

A $10 \mathrm{~mL}$ round bottom flask and a stir bar were oven-dried and brought into a glove box. $\mathrm{Ni}(\mathrm{cod})_{2}(28 \mathrm{mg}, 0.1 \mathrm{mmol}, 20 \mathrm{~mol} \%$ ), dicyclohexylphenylphosphine (56 mg, $0.2 \mathrm{mmol}, 40$ $\mathrm{mol} \%$ ) and 2-naphthaldehyde (78 $\mathrm{mg}, 0.5 \mathrm{mmol}, 100 \mathrm{~mol} \%$ ) were added to the round bottom flask, the flask was sealed with a septum, and the sealed flask was brought out of the glove box and connected to an argon line. The catalyst mixture was dissolved in toluene $(2.5 \mathrm{~mL})$ under argon and stirred $5 \mathrm{~min}$ at room temperature. The system was purged with propene for $1 \mathrm{~min}$. The propene atmosphere was maintained by a propene balloon. Triethylamine $(418 \mu \mathrm{L}, 3 \mathrm{mmol}$, $600 \mathrm{~mol} \%)$ was added. TESOTf (197 $\mu \mathrm{L}, 0.875 \mathrm{mmol}, 175 \mathrm{~mol} \%)$ was added. The mixture was stirred at room temperature for $6 \mathrm{~h}$. The mixture was diluted with hexane and filtered through a plug of silica gel. Solvent was removed under reduced pressure. Purification via flash chromatography on silica afforded $\mathbf{2} \mathbf{r}^{\prime}$ in $73 \%$ isolated yield as a colorless oil and $\mathbf{2 r}$ in $14 \%$ isolated yield as a colorless oil.

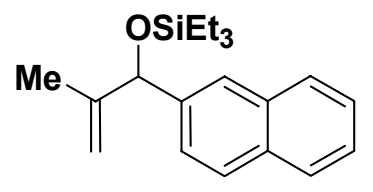

$\left(2 \mathbf{r}^{\prime}\right)$

${ }^{1} \mathrm{H}$ NMR (400 MHz, $\left.\mathrm{CDCl}_{3}, \delta\right): 7.86(\mathrm{~m}, 4 \mathrm{H}) ; 7.50(\mathrm{~m}, 3 \mathrm{H}) ; 5.33(\mathrm{~s}, 1 \mathrm{H}) ; 5.26(\mathrm{~s}, 1 \mathrm{H}) ; 4.94$ (s, $1 \mathrm{H}) ; 1.62(\mathrm{~s}, 3 \mathrm{H}) ; 1.00(\mathrm{t}, J=8.0 \mathrm{~Hz}, 9 \mathrm{H}) ; 0.67$ (dq, $J=1.8,7.9 \mathrm{~Hz}, 6 \mathrm{H})$.

${ }^{13} \mathrm{C}$ NMR (100 MHz, $\left.\mathrm{CDCl}_{3}, \delta\right): 148.0,141.0,133.4,133.0,128.2,127.8,127.8,126.0,125.7$, 124.9, 124.8, 78.6, 17.6, 7.1, 5.1.

IR (NaCl, thin film): 2955, 2912, 2876, 1652, 1508, 1457, 1238, 1084, 1005, 899, 742.

HRMS-ESI (m / z): [M + Na $]^{+}$calcd for $\mathrm{C}_{20} \mathrm{H}_{28} \mathrm{OSiNa}$, 335.180; found, 335.181. 


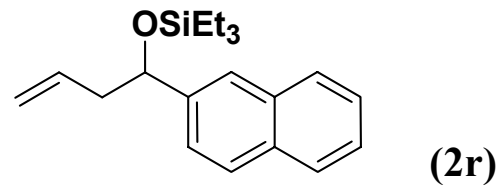

${ }^{1} \mathrm{H}$ NMR (400 MHz, $\left.\mathrm{CDCl}_{3}, \delta\right): 7.83(\mathrm{t}, J=8.5 \mathrm{~Hz}, 3 \mathrm{H}) ; 7.75(\mathrm{~s}, 1 \mathrm{H}) ; 7.48(\mathrm{~m}, 3 \mathrm{H}) ; 5.81(\mathrm{~m}$, 1H); $5.05(\mathrm{~m}, 1 \mathrm{H}) ; 5.02(\mathrm{~m}, 1 \mathrm{H}) ; 4.86(\mathrm{t}, J=5.9 \mathrm{~Hz}) ; 2.55(\mathrm{~m}, 2 \mathrm{H}) ; 0.91(\mathrm{t}, J=8.0 \mathrm{~Hz}, 9 \mathrm{H}) ; 0.57$ (dq, $J=3.5,7.5 \mathrm{~Hz}, 6 \mathrm{H})$.

${ }^{13} \mathrm{C}$ NMR (100 MHz, $\left.\mathrm{CDCl}_{3}, \delta\right): 142.8,135.3,133.4,133.0,128.1,127.9,127.9,126.1,125.7$, 124.6, 75.2, 45.6, 7.0, 5.0.

IR (NaCl, thin film): 2955, 2876, 1458, 1239, 1084, 1005, 914, 817, 743.

HRMS-ESI (m / z): [M + Na] $]^{+}$calcd for $\mathrm{C}_{20} \mathrm{H}_{28} \mathrm{OSiNa}$, 335.180; found, 335.181. 


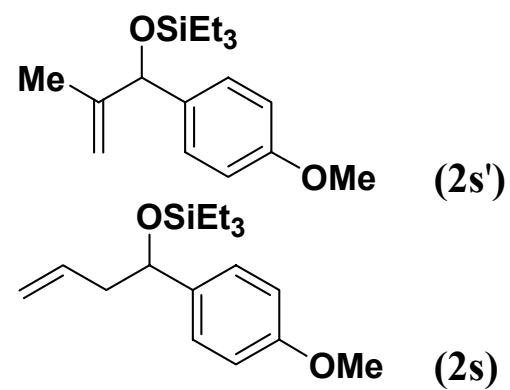

The reaction of propene (1atm, balloon) and $p$-anisaldehyde $(61 \mu \mathrm{L}, 0.5 \mathrm{mmol})$ with $\mathrm{Ni}(\operatorname{cod})_{2}$, $\mathrm{Cy}_{2} \mathrm{PhP}$ (56 mg, $0.2 \mathrm{mmol}, 40 \mathrm{~mol} \%$ ) and TESOTf (197 $\left.\mu \mathrm{L}, 0.875 \mathrm{mmol}\right)$, triethylamine in toluene following the procedure for $\mathbf{2} \mathbf{r}^{\prime}$ above afforded $\mathbf{2} \mathbf{s}^{\prime}$ and $\mathbf{2 s}$ and the ratio of $\mathbf{2} \mathbf{s}^{\prime}: \mathbf{2 s}$ is $82: 18$. Purification via flash chromatography on silica afforded $2 s^{\prime}$ and $2 s$ as a colorless mixture in $95 \%$ isolated yield.

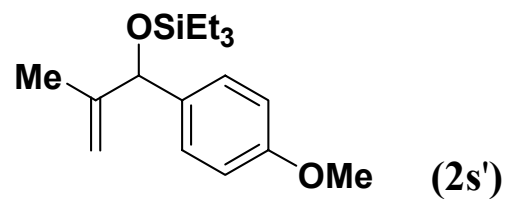

${ }^{1} \mathrm{H}$ NMR (400 MHz, $\left.\mathrm{CDCl}_{3}, \delta\right): 7.30(\mathrm{~d}, J=8.7 \mathrm{~Hz}, 2 \mathrm{H}) ; 6.90(\mathrm{~d}, J=8.7 \mathrm{~Hz}, 2 \mathrm{H}) ; 5.22(\mathrm{~s}, 1 \mathrm{H})$; $5.08(\mathrm{~s}, 1 \mathrm{H}) ; 4.96(\mathrm{~s}, 1 \mathrm{H}) ; 3.62(\mathrm{~s}, 3 \mathrm{H}) ; 2.15(\mathrm{~s}, 1 \mathrm{H}) ; 1.62(\mathrm{~s}, 3 \mathrm{H})$.

${ }^{13} \mathrm{C} \mathrm{NMR}\left(100 \mathrm{MHz}, \mathrm{CDCl}_{3}, \delta\right): 159.3,147.2,134.3,127.9,113.9,110.8,77.5,55.4,18.7$.

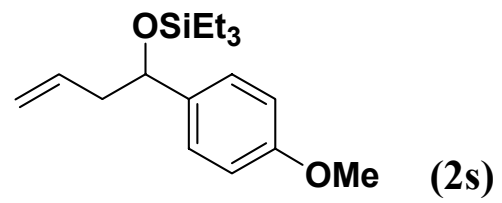

${ }^{1} \mathrm{H}$ NMR (400 MHz, $\left.\mathrm{CDCl}_{3}, \delta\right): 7.30(\mathrm{~d}, J=8.7 \mathrm{~Hz}, 2 \mathrm{H}) ; 6.90(\mathrm{~d}, J=8.7 \mathrm{~Hz}, 2 \mathrm{H}) ; 5.82(\mathrm{~m}, 1 \mathrm{H})$; $5.15(\mathrm{~m}, 2 \mathrm{H}) ; 4.69(\mathrm{t}, J=6.5 \mathrm{~Hz}, 1 \mathrm{H}) ; 2.52(\mathrm{~d}, J=6.8 \mathrm{~Hz}, 2 \mathrm{H}), 2.15(\mathrm{~s}, 1 \mathrm{H})$.

${ }^{13} \mathrm{C}$ NMR (100 MHz, $\left.\mathrm{CDCl}_{3}, \delta\right): 159.1,136.2,134.8,127.3,118.4,113.9,73.1,55.4,43.9$. 


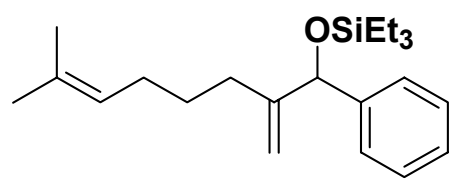

$\left(2 \mathbf{t}^{\prime}\right)$

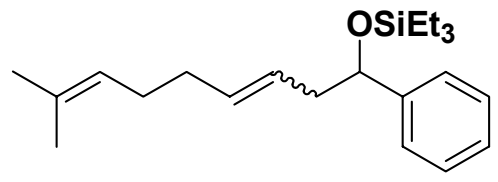

\section{(2t)}

A $10 \mathrm{~mL}$ round bottom flask and a stir bar were oven-dried and brought into a glove box. $\mathrm{Ni}(\operatorname{cod})_{2}(27.5 \mathrm{mg}, 0.2 \mathrm{mmol}, 20 \mathrm{~mol} \%)$ and dicyclohexylphenylphosphine $(55 \mathrm{mg}, 0.4 \mathrm{mmol}$, $40 \mathrm{~mol} \%$ ) were added to the round bottom flask, the flask was sealed with a septum, and the sealed flask was brought out of the glove box and connected to an argon line. The catalyst mixture was dissolved in toluene $(1.0 \mathrm{~mL})$ under argon and stirred $5 \mathrm{~min}$ at room temperature. 7-methyl-1,7-octa- diene ( $825 \mu \mathrm{L}, 5 \mathrm{mmol}, 1000 \mathrm{~mol} \%)$ was added. Triethylamine (418 $\mu \mathrm{L}, 3$ mmol, $600 \mathrm{~mol} \%)$ was added. TESOTf $(197 \mu \mathrm{L}, 0.875 \mathrm{mmol}, 175 \mathrm{~mol} \%)$ was added. Benzaldehyde $(51 \mu \mathrm{L}, 0.5 \mathrm{mmol}, 100 \mathrm{~mol} \%)$ in $1.5 \mathrm{~mL}$ toluene was added to the reaction mixture over $6 \mathrm{~min}$. The mixture was stirred at room temperature for $18 \mathrm{~h}$. The mixture was filtered through a plug of silica gel. Solvent was removed under reduced pressure and ${ }^{1} \mathrm{H}$ NMR of the crude mixture indicated the ratio of $\mathbf{2} \mathbf{t}^{\prime}: \mathbf{2 t}$ is $71: 29$. Purification via flash chromatography on silica afforded $2 \mathbf{t}^{\prime}$ in $50 \%$ isolated yield as a colorless oil and $\mathbf{2 t}$ in $22 \%$ isolated yield as a colorless oil.

This reaction can be run according to general procedure 3, which also afforded $2 \mathbf{t}^{\prime}$ and $2 \mathbf{t}$ in similar yield.

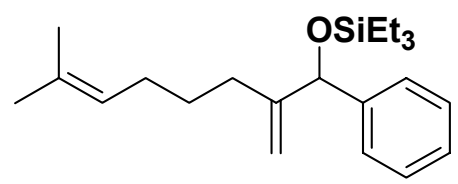

(2t')

${ }^{1} \mathrm{H} \mathrm{NMR}\left(400 \mathrm{MHz}, \mathrm{CDCl}_{3}, \delta\right): 7.40(\mathrm{~d}, J=7.0 \mathrm{~Hz}, 2 \mathrm{H}) ; 7.34(\mathrm{t}, J=7.8 \mathrm{~Hz}, 2 \mathrm{H}) ; 7.27$ (t, $J=7.2$, 1H); 5.26 (bs, 1H); 5.18 (bs, 1H); 5.10 (t, $J=7.2 \mathrm{~Hz}, 1 \mathrm{H}) ; 4.81$ (bs, 1H); $1.76-2.10$ (m, 4H); $1.71(\mathrm{~s}, 3 \mathrm{H}) ; 1.60(\mathrm{~s}, 3 \mathrm{H}) ; 1.44$ (quintet, $J=7.7 \mathrm{~Hz}, 2 \mathrm{H}) ; 0.97$ (t, $J=7.9 \mathrm{~Hz}, 9 \mathrm{H}) ; 0.62$ (dq, $J=$ $1.5,7.9 \mathrm{~Hz}, 6 \mathrm{H})$.

${ }^{13} \mathrm{C}$ NMR (100 MHz, $\left.\mathrm{CDCl}_{3}, \delta\right): 152.1,143.7,131.6,128.1,127.1,126.6,124.8,109.5,78.2$, $30.4,28.2,28.1,25.9,17.8,7.0,5.0$.

IR (NaCl, thin film): 2955, 2877, 1647, 1456, 1091, 1067, 743.

HRMS-ESI (m / z): [M + Na] $]^{+}$calcd for $\mathrm{C}_{22} \mathrm{H}_{36} \mathrm{OSiNa}$, 367.243; found, 367.243 . 


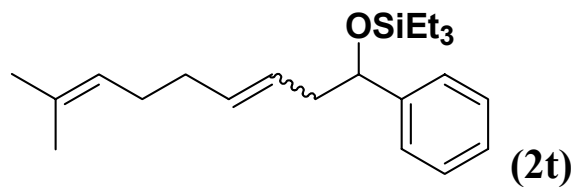

${ }^{1} \mathrm{H}$ NMR (400 MHz, $\left.\mathrm{CDCl}_{3}, \delta\right): 7.30(\mathrm{~m}, 5 \mathrm{H}) ; 5.45(\mathrm{~m}, 2 \mathrm{H}) ; 5.15(\mathrm{t}, J=7.1 \mathrm{~Hz}, 1 \mathrm{H}) ; 4.64(\mathrm{dd}, J$ $=5.4,7.3 \mathrm{~Hz}, 1 \mathrm{H}$ ); 2.45 (quintet, $J=5.4 \mathrm{~Hz}, 1 \mathrm{H}$ ); 2.35 (quintet, $J=5.9 \mathrm{~Hz}, 1 \mathrm{H}$ ); $2.05(\mathrm{~m}, 4 \mathrm{H}$ ); $1.62(\mathrm{~s}, 3 \mathrm{H}) ; 1.72(\mathrm{~s}, 3 \mathrm{H}) ; 0.92(\mathrm{t}, J=7.9 \mathrm{~Hz}, 9 \mathrm{H}) ; 0.55(\mathrm{dq}, J=1.5,7.9 \mathrm{~Hz}, 6 \mathrm{H})$.

${ }^{13} \mathrm{C}$ NMR $\left(100 \mathrm{MHz}, \mathrm{CDCl}_{3}, \delta\right): 145.6,132.8,131.7,128.1,127.1,126.9,126.1,124.4,75.6$, $44.5,33.1,28.2,25.9,17.9,7.0,5.0$.

IR (NaCl, thin film): 2955, 2914, 2876, 1454, 1089, 1005, 969, 699.

HRMS-ESI (m / z): [M + Na] $]^{+}$calcd for $\mathrm{C}_{22} \mathrm{H}_{36} \mathrm{OSiNa}$, 367.243; found, 367.243. 


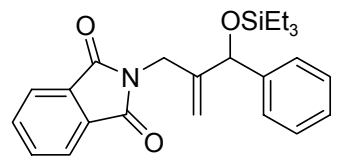

(4a)

The reaction of allylphthalimide ( $281 \mathrm{mg}, 1.5 \mathrm{mmol}, 300 \mathrm{~mol} \%)$ and benzaldehyde $(51 \mu \mathrm{L}, 0.5$ mmol) with $\mathrm{Ni}(\operatorname{cod})_{2}$, dicyclohexylphenylphosphine (55 mg, $\left.0.4 \mathrm{mmol}, 40 \mathrm{~mol} \%\right)$ and TESOTf $(197 \mu \mathrm{L}, 0.875 \mathrm{mmol})$, triethylamine in toluene at $35^{\circ} \mathrm{C}$ following the general procedure 3 above afforded $\mathbf{4 a}$ and $\mathbf{4 a}$ ' in $67 \%$ total yield according to ${ }^{1} \mathrm{H}$ NMR of the crude mixture and the ratio of 4a:4a' is 74:26. Purification via flash chromatography on silica afforded $4 \mathbf{a}$ as a mixture of $\mathbf{4 a}$ and the isomerized starting material.

${ }^{1} \mathrm{H}$ NMR (400 MHz, $\mathrm{CDCl}_{3}, \delta$ ): 7.77 (dd, $\left.J=3.0,5.4 \mathrm{~Hz}, 2 \mathrm{H}\right) ; 7.73(\mathrm{dd}, J=3.0,5.4 \mathrm{~Hz}, 2 \mathrm{H})$; 7.13-7.41 (m, 5H); $5.36(\mathrm{~s}, 1 \mathrm{H}), 5.30(\mathrm{~s}, 1 \mathrm{H}), 4.99(\mathrm{~s}, 1 \mathrm{H}), 4.26(\mathrm{~d}, J=16 \mathrm{~Hz}, 1 \mathrm{H}), 4.08(\mathrm{~d}, J=$ $16 \mathrm{~Hz}, 1 \mathrm{H}), 0.91(\mathrm{t}, J=7.9 \mathrm{~Hz}, 9 \mathrm{H}) ; 0.59$ (q, $J=7.9 \mathrm{~Hz}, 6 \mathrm{H})$.

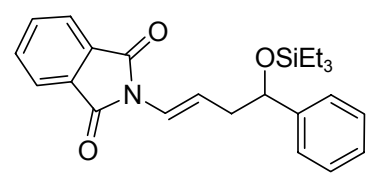

(4a').

The reaction of allylphthalimide ( $1.5 \mathrm{mmol}, 300 \mathrm{~mol} \%)$ and benzaldehyde $(51 \mu \mathrm{L}, 0.5 \mathrm{mmol})$ with $\mathrm{Ni}(\mathrm{cod})_{2}, \mathrm{EtOPh}_{2} \mathrm{P}(43 \mu \mathrm{l}, 0.2 \mathrm{mmol}, 40 \mathrm{~mol} \%)$ and TESOTf $(197 \mu \mathrm{L}, 0.875 \mathrm{mmol})$, triethylamine in toluene at $35^{\circ} \mathrm{C}$ following the general procedure 3 above afforded $\mathbf{4 a}$ and $\mathbf{4 a}$ ' in $43 \%$ total yield according to ${ }^{1} \mathrm{H}$ NMR of the crude mixture and the ratio of $\mathbf{4 a}: 4 \mathbf{a}^{\prime}$ is $12: 88$. The $E$ $/ Z$ ratio of $\mathbf{4} \mathbf{a}^{\prime}$ is $60: 40$. Purification via flash chromatography on silica afforded $\mathbf{4 a}$ ' as a mixture with the isomerized starting material.

${ }^{1} \mathrm{H}$ NMR (400 MHz, $\mathrm{CDCl}_{3}, \delta$ ): $7.86(\mathrm{dd}, J=3.1,5.4 \mathrm{~Hz}, 2 \mathrm{H}) ; 7.73$ (dd, $\left.J=3.1,5.4 \mathrm{~Hz}, 2 \mathrm{H}\right)$; $7.25-7.37(\mathrm{~m}, 5 \mathrm{H}) ; 6.62(\mathrm{~m}, 2 \mathrm{H}) ; 4.76(\mathrm{dd}, J=5.5,6.9 \mathrm{~Hz}, 1 \mathrm{H}) ; 2.47-2.60(\mathrm{~m}, 2 \mathrm{H}) ; 0.89(\mathrm{t}, J=$ $8.0 \mathrm{~Hz}, 9 \mathrm{H}) ; 0.56(\mathrm{q}, J=2.8,8.0 \mathrm{~Hz}, 6 \mathrm{H})$.

${ }^{13} \mathrm{C}$ NMR (100 MHz, $\left.\mathrm{CDCl}_{3}, \delta\right): 166.7,149.2,145.0,134.5,131.9,128.2,127.3,126.1,123.7$, 119.5, 118.8, 75.0, 43.1, 7.0, 5.0.

IR ( $\mathrm{NaCl}$, thin film): 2954, 2876, 1781, 1721, 1384, 1088, 1069, 715, 701.

HRMS-ESI (m/z): [M-OTES] $]^{+}$calcd for $\mathrm{C}_{18} \mathrm{H}_{14} \mathrm{NO}_{2} \mathrm{Na}, 276.1025$; found, 276.1022 . 


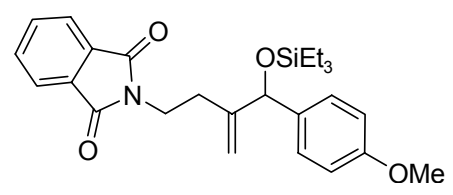

(4b)

The reaction of homoallylphthalimide $(1.5 \mathrm{mmol}, 300 \mathrm{~mol} \%)$ and $o$-anisaldehyde $(61 \mu \mathrm{L}, 0.5$ mmol) with $\mathrm{Ni}(\mathrm{cod})_{2}$, dicyclohexylphenylphosphine (55 $\left.\mathrm{mg}, 0.4 \mathrm{mmol}, 40 \mathrm{~mol} \%\right)$ and TESOTf $(197 \mu \mathrm{L}, 0.875 \mathrm{mmol})$, triethylamine in toluene at $35^{\circ} \mathrm{C}$ following the general procedure 3 above afforded $\mathbf{4 b}$ and $\mathbf{4} \mathbf{b}^{\prime}$ in $54 \%$ total yield according to ${ }^{1} \mathrm{H}$ NMR of the crude mixture and the ratio of $\mathbf{4 b}: \mathbf{4} \mathbf{b}^{\prime}$ is $71: 29$. Purification via flash chromatography on silica afforded $\mathbf{4 b}$ and $\mathbf{4} \mathbf{b}$ '. ${ }^{1} \mathrm{H}$ NMR (400 MHz, $\left.\mathrm{CDCl}_{3}, \delta\right): 7.81(\mathrm{dd}, J=3.0,5.4,2 \mathrm{H}) ; 7.70(\mathrm{dd}, J=3.0,5.4,2 \mathrm{H}) ; 7.26(\mathrm{~d}, J$ $=8.7 \mathrm{~Hz}, 2 \mathrm{H}) ; 6.79(\mathrm{~d}, J=8.7 \mathrm{~Hz}, 2 \mathrm{H}) ; 5.27(\mathrm{~s}, 1 \mathrm{H}) ; 5.15(\mathrm{~s}, 1 \mathrm{H}) ; 4.99(\mathrm{~s}, 1 \mathrm{H}) ; 3.66-3.86(\mathrm{~m}$, $2 \mathrm{H}) ; 3.78(\mathrm{~s}, 3 \mathrm{H}) ; 2.33-2.40(\mathrm{~m}, 1 \mathrm{H}) ; 2.16-2.23(\mathrm{~m}, 1 \mathrm{H}) ; 0.90(\mathrm{t}, J=7.9 \mathrm{~Hz}, 9 \mathrm{H}) ; 0.57$ (q, $J=$ $7.9 \mathrm{~Hz}, 6 \mathrm{H})$.

${ }^{13} \mathrm{C}$ NMR $\left(100 \mathrm{MHz}, \mathrm{CDCl}_{3}, \delta\right): 168.4,158.8,148.6,135.2,134.0,132.3,127.7,123.3,113.5$, $111.8,77.6,55.3,37.2,29.8,7.0,5.0$.

IR ( $\mathrm{NaCl}$, thin film): 2954, 2876, 1773, 1715, 1511, 1467, 1431, 1395, 1354, 1247, 1078, 952 , 719.

HRMS-ESI (m / z): [M + Na] $]^{+}$calcd for $\mathrm{C}_{26} \mathrm{H}_{33} \mathrm{O}_{4} \mathrm{SiNa}$, 474.2066; found, 474.2071. 


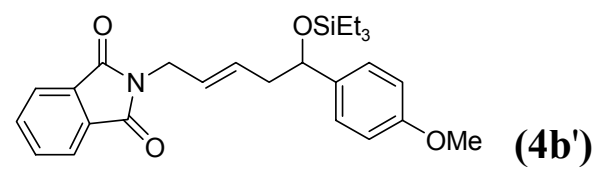

The reaction of homoallylphthalimide $(1.5 \mathrm{mmol}, 300 \mathrm{~mol} \%)$ and $o$-anisaldehyde $(61 \mu \mathrm{L}, 0.5$ mmol) with $\mathrm{Ni}(\mathrm{cod})_{2}, \mathrm{Ph}_{3} \mathrm{P}(52 \mathrm{mg}, 0.2 \mathrm{mmol}, 40 \mathrm{~mol} \%)$ and TESOTf (197 $\left.\mu \mathrm{L}, 0.875 \mathrm{mmol}\right)$, triethylamine in toluene at $35^{\circ} \mathrm{C}$ following the general procedure 3 above afforded $4 \mathbf{b}$ and $4 \mathbf{b}$ ' in $76 \%$ total yield according to ${ }^{1} \mathrm{H}$ NMR of the crude mixture and the ratio of $\mathbf{4 b}: \mathbf{b} \mathbf{b}$ ' is $<5: 95$. Treatment of $4 b^{\prime}$ with TBAF followed by flash chromatography on silica afforded a desilylated $4 b^{\prime}$.

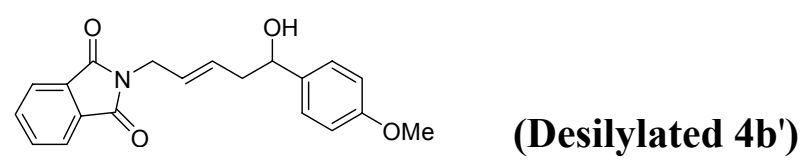

${ }^{1} \mathrm{H}$ NMR (400 MHz, $\left.\mathrm{CDCl}_{3}, \delta\right): 7.86(\mathrm{dd}, J=3.1,5.4 \mathrm{~Hz}, 2 \mathrm{H}) ; 7.73(\mathrm{dd}, J=3.1,5.4 \mathrm{~Hz}, 2 \mathrm{H})$; $7.25(\mathrm{~d}, J=8.7 \mathrm{~Hz}, 2 \mathrm{H}) ; 6.84(\mathrm{~d}, J=8.7 \mathrm{~Hz}, 2 \mathrm{H}) ; 5.73(\mathrm{dt}, J=6.0,15.4 \mathrm{~Hz}, 1 \mathrm{H}) ; 5.62(\mathrm{dt}, J=6.0$, $15.4 \mathrm{~Hz}, 1 \mathrm{H}) ; 4.68$ (dd, $J=6.4,6.4 \mathrm{~Hz}, 1 \mathrm{H}) ; 4.25-4.33$ (m, 2H); 3.78 (s, 3H); 2.46 (m, 2H), 2.09 (brs, $1 \mathrm{H})$.

${ }^{13} \mathrm{C}$ NMR (100 MHz, $\left.\mathrm{CDCl}_{3}, \delta\right): 168.2,159.1,136.1,134.1,132.3,130.8,127.2,127.1,123.5$, $113.9,73.1,55.4,42.3,39.7$.

IR (NaCl, thin film): 3466, 2929, 1770, 1711, 1611, 1512, 1395, 1249, 1174, 1034, 833, 720.

HRMS-ESI (m / z): [M + Na] $]^{+}$calcd for $\mathrm{C}_{20} \mathrm{H}_{18} \mathrm{NO}_{3} \mathrm{Na}, 320.129$; found, 320.130 . 


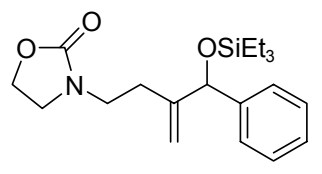

(4c)

The reaction of homoallyloxazolidinone $(1.5 \mathrm{mmol}, 300 \mathrm{~mol} \%)$ and benzaldehyde $(51 \mu \mathrm{L}, 0.5$ mmol) with $\mathrm{Ni}(\mathrm{cod})_{2}$, dicyclohexylphenylphosphine (55 mg, $0.4 \mathrm{mmol}, 40 \mathrm{~mol} \%$ ) and TESOTf $(197 \mu \mathrm{L}, 0.875 \mathrm{mmol})$, triethylamine in toluene at room temperature following the general procedure 3 above afforded $\mathbf{4} \mathbf{c}$ and $\mathbf{4} \mathbf{c}^{\prime}$ in $60 \%$ total yield according to ${ }^{1} \mathrm{H}$ NMR of the crude

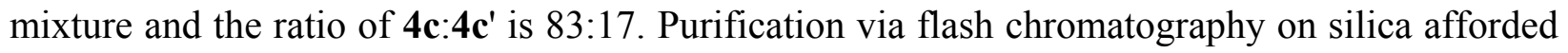
$4 \mathbf{c}$ and $4 \mathbf{c}^{\prime}$ as colorless oils.

${ }^{1} \mathrm{H}$ NMR (400 MHz, $\left.\mathrm{CDCl}_{3}, \delta\right): 7.23-7.38(\mathrm{~m}, 5 \mathrm{H}) ; 5.31(\mathrm{~s}, 1 \mathrm{H}) ; 5.20(\mathrm{~s}, 1 \mathrm{H}) ; 5.00(\mathrm{~s}, 1 \mathrm{H})$; $4.16-4.21(\mathrm{~m}, 2 \mathrm{H}) ; 3.19-3.36(\mathrm{~m}, 4 \mathrm{H}) ; 2.02-2.26$ (m, 2H); 0.93 (t, $J=7.9 \mathrm{~Hz}, 9 \mathrm{H}) ; 0.60$ (q, $J=$ $7.8 \mathrm{~Hz}, 6 \mathrm{H})$.

${ }^{13} \mathrm{C}$ NMR $\left(100 \mathrm{MHz}, \mathrm{CDCl}_{3}, \delta\right): 158.4,148.1,143.1,128.2,127.4,126.3,112.0,78.1,61.8,44.3$, $42.8,27.9,7.0,4.9$.

IR ( $\mathrm{NaCl}$, thin film): 2955, 2912, 2876, 1753, 1484, 1426, 1265, 1089, 1067, 1044, 1007, 861, $744,701$.

HRMS-ESI (m/z): [M+Na] $]^{+}$calcd for $\mathrm{C}_{20} \mathrm{H}_{31} \mathrm{NO}_{3} \mathrm{Na}, 384.1965$; found, 384.1951 . 
<smiles>CCOC(CC=CCN1CCOC1=O)c1ccccc1</smiles>

The reaction of homoallylphthalimide $(1.5 \mathrm{mmol}, 300 \mathrm{~mol} \%)$ and benzaldehyde $(51 \mu \mathrm{L}, 0.5$ mmol) with $\mathrm{Ni}(\operatorname{cod})_{2}, \mathrm{EtOPh}_{2} \mathrm{P}(43 \mu \mathrm{l}, 0.2 \mathrm{mmol}, 40 \mathrm{~mol} \%)$ and TESOTf (197 $\left.\mu \mathrm{L}, 0.875 \mathrm{mmol}\right)$, triethylamine in toluene at room temperature following the general procedure 3 above afforded

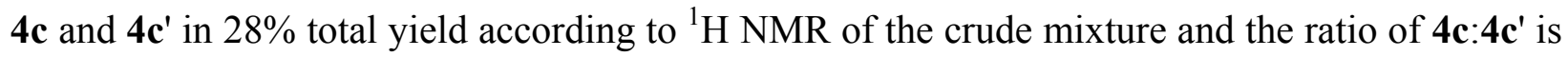
10:90. $4 \mathrm{c}^{\prime}$ was subjected to TBAF and purification via flash chromatography on silica afforded a desilylated $\mathbf{4} \mathbf{c}^{\prime}$.<smiles>O=C1OCCN1C/C=C/CC(O)c1ccccc1</smiles>

\section{(Desilylated 4c')}

${ }^{1} \mathrm{H}$ NMR (400 MHz, $\left.\mathrm{CDCl}_{3}, \delta\right): 7.28-7.42(\mathrm{~m}, 5 \mathrm{H}), 5.68(\mathrm{dt}, J=5.7,7.1 \mathrm{~Hz}, 1 \mathrm{H}), 5.49(\mathrm{dt}, J=$ $5.7,7.1 \mathrm{~Hz}, 1 \mathrm{H}), 4.77(\mathrm{dd}, J=6.7,6.8 \mathrm{~Hz}, 1 \mathrm{H}), 4.28(\mathrm{t}, J=8.0 \mathrm{~Hz}, 2 \mathrm{H}), 3.80-3.82(\mathrm{~m}, 2 \mathrm{H}), 3.38$ (dt, $J=2.5,8.0 \mathrm{~Hz}, 2 \mathrm{H}), 2.53-2.59$ (m, 2H), 2.11-2.17 (brs, $1 \mathrm{H}$ ).

${ }^{13} \mathrm{C}$ NMR (100 MHz, $\left.\mathrm{CDCl}_{3}, \delta\right): 158.4,143.9,131.2,128.7,127.8,127.3,126.0,73.8,61.9,46.4$, 44.2, 42.1 .

IR (NaCl, thin film): 3421, 2919, 2361, 1734, 1653, 1490, 1437, 1259, 1038, 762, 702.

HRMS-ESI (m/z): [M+Na $]^{+}$calcd for $\mathrm{C}_{14} \mathrm{H}_{17} \mathrm{NO}_{3} \mathrm{Na}, 270.111$; found, 270.110 . 


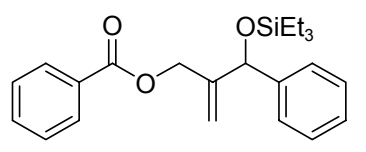

(4d)

The reaction of allylbenzoate $(2.5 \mathrm{mmol}, 500 \mathrm{~mol} \%)$ and benzaldehyde $(51 \mu \mathrm{L}, 0.5 \mathrm{mmol})$ with $\mathrm{Ni}(\operatorname{cod})_{2}$, dicyclohexylphenylphosphine (55 mg, $\left.0.4 \mathrm{mmol}, 40 \mathrm{~mol} \%\right)$ and TESOTf (197 $\mu \mathrm{L}$, $0.875 \mathrm{mmol}$ ), triethylamine in toluene following the general procedure 3 above afforded $\mathbf{4 d}$ and $\mathbf{4 d}$ ' in $<5 \%$ total yield according to ${ }^{1} \mathrm{H}$ NMR of the crude mixture. $4 \mathbf{d}$ and $\mathbf{4 d}$ ' were not isolated from the reaction mixture.

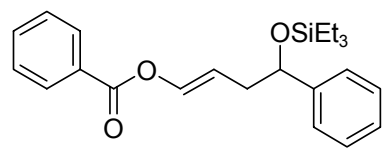

$\left(4 d^{\prime}\right)$

The reaction of allylbenzoate $(2.5 \mathrm{mmol}, 500 \mathrm{~mol} \%)$ and benzaldehyde $(51 \mu \mathrm{L}, 0.5 \mathrm{mmol})$ with $\mathrm{Ni}(\mathrm{cod})_{2}, \mathrm{EtOPh}_{2} \mathrm{P}(43 \mu \mathrm{l}, 0.2 \mathrm{mmol}, 40 \mathrm{~mol} \%)$ and TESOTf $(197 \mu \mathrm{L}, 0.875 \mathrm{mmol})$, triethylamine in toluene at $35{ }^{\circ} \mathrm{C}$ following the general procedure 3 above afforded $\mathbf{4 d}$ and $\mathbf{4 d}$ in $<5 \%$ total yield according to ${ }^{1} \mathrm{H}$ NMR of the crude mixture. $\mathbf{4 d}$ and $\mathbf{4 d}$ ' were not isolated from the reaction mixture. 


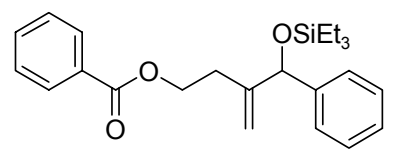

(4e)

The reaction of homoallylbenzoate $(1.5 \mathrm{mmol}, 300 \mathrm{~mol} \%)$ and benzaldehyde $(51 \mu \mathrm{L}, 0.5 \mathrm{mmol})$ with $\mathrm{Ni}(\mathrm{cod})_{2}$, dicyclohexylphenylphosphine (55 mg, $\left.0.4 \mathrm{mmol}, 40 \mathrm{~mol} \%\right)$ and TESOTf (197 $\mu \mathrm{L}$, $0.875 \mathrm{mmol}$ ), triethylamine in toluene at room temperature following the general procedure 3 above afforded $\mathbf{4 e}$ and $\mathbf{4} \mathbf{e}^{\prime}$ in $21 \%$ total yield according to ${ }^{1} \mathrm{H}$ NMR of the crude mixture. $4 \mathbf{e}$ was subjected to TBAF and the free alcohol was isolated as a colorless oil.

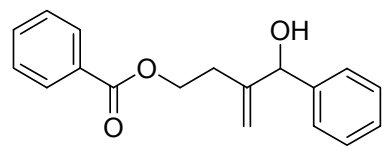

\section{(Desilylated 4e)}

${ }^{1} \mathrm{H}$ NMR (400 MHz, $\left.\mathrm{CDCl}_{3}, \delta\right): 8.02(\mathrm{~d}, J=7.3 \mathrm{~Hz}, 2 \mathrm{H}) ; 7.58(\mathrm{t}, J=7.3 \mathrm{~Hz}, 1 \mathrm{H}) ; 7.28(\mathrm{~m}, 7 \mathrm{H})$; 5.37 (s, 1H); 5.29 (s, 1H); $5.12(\mathrm{~s}, 1 \mathrm{H}) ; 4.36-4.50(\mathrm{~m}, 2 \mathrm{H}) ; 2.34-2.51$ (m, 2H); 2.29 (brs, 1H). ${ }^{13} \mathrm{C}$ NMR (100 MHz, $\left.\mathrm{CDCl}_{3}, \delta\right): 166.9,147.0,141.8,133.1,130.4,129.7,128.7,128.5,128.0$, 126.7, 113.3, 77.6, 63.7, 31.3.

IR ( $\mathrm{NaCl}$, thin film): 3447, 3063, 3030, 2961, 1717, 1701, 1451, 1316, 1276, 1117, 1071, 1026, 912, 712, 701, 668.

HRMS-ESI (m/z): [M+Na $]^{+}$calcd for $\mathrm{C}_{18} \mathrm{H}_{18} \mathrm{O}_{3} \mathrm{Na}, 305.1148$; found, 305.1156 . 


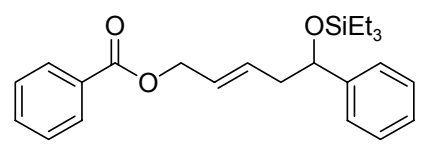

$\left(4 e^{\prime}\right)$

The reaction of homoallylbenzoate $(1.5 \mathrm{mmol}, 300 \mathrm{~mol} \%)$ and benzaldehyde $(51 \mu \mathrm{L}, 0.5 \mathrm{mmol})$ with $\mathrm{Ni}(\mathrm{cod})_{2}, \mathrm{Ph}_{3} \mathrm{P}(52 \mathrm{mg}, 0.2 \mathrm{mmol}, 40 \mathrm{~mol} \%)$ and TESOTf $(197 \mu \mathrm{L}, 0.875 \mathrm{mmol})$, triethylamine in toluene at $35^{\circ} \mathrm{C}$ following the general procedure 3 above afforded $4 \mathbf{e}$ and $\mathbf{4 e}$ ' in $<5 \%$ total yield according to ${ }^{1} \mathrm{H}$ NMR of the crude mixture and. $4 \mathrm{e}^{\prime}$ was not isolated from the reaction mixture.

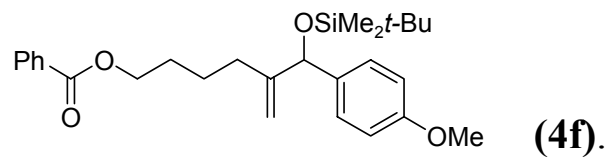

The reaction of 1-hexen-6-benzoate ( $510.3 \mathrm{mg}, 2.5 \mathrm{mmol}, 500 \mathrm{~mol} \%)$ and $o$-anisaldehyde $(61 \mu \mathrm{L}$, $0.5 \mathrm{mmol})$ with $\mathrm{Ni}(\mathrm{cod})_{2}, \mathrm{EtOPh}_{2} \mathrm{P}(43 \mu \mathrm{l}, 0.2 \mathrm{mmol}, 40 \mathrm{~mol} \%)$ and TESOTf $(197 \mu \mathrm{L}, 0.875$ $\mathrm{mmol}, 175 \mathrm{~mol} \%$ ), triethylamine in toluene following the general procedure 3 above afforded $\mathbf{4 f}$ and $\mathbf{4} \mathbf{f}^{\prime}$ in $44 \%$ total isolated yield after flash chromatography on silica and according to ${ }^{1} \mathrm{H}$ NMR

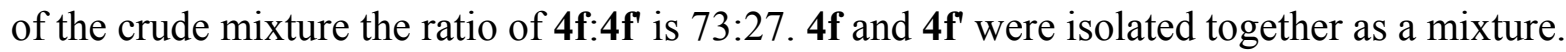




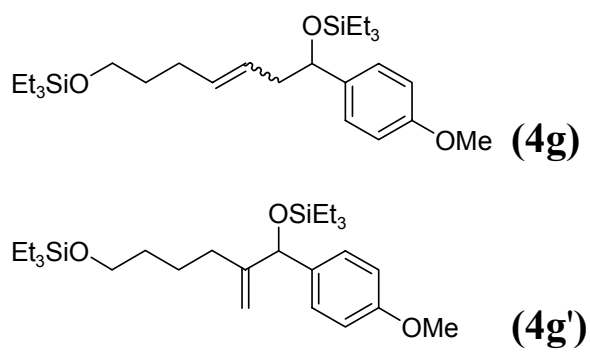

The reaction of triethyl-hex-5-enyloxy-silane $(1.5 \mathrm{mmol}, 300 \mathrm{~mol} \%)$ and $o$-anisaldehyde $(61 \mu \mathrm{L}$, $0.5 \mathrm{mmol})$ with $\mathrm{Ni}(\mathrm{cod})_{2}, \mathrm{EtOPh}_{2} \mathrm{P}(43 \mu \mathrm{l}, 0.2 \mathrm{mmol}, 40 \mathrm{~mol} \%)$ and TESOTf $(197 \mu \mathrm{L}, 0.875$ $\mathrm{mmol}, 175 \mathrm{~mol} \%$ ), triethylamine in toluene at $35{ }^{\circ} \mathrm{C}$ following the general procedure 3 above afforded $\mathbf{4 g}$ and $\mathbf{4} \mathbf{g}$ in $66 \%$ total yield according to ${ }^{1} \mathrm{H}$ NMR of the crude mixture and the ratio of $\mathbf{4 g : 4 g ^ { \prime }}$ is $92: 8$. The $E / Z$ ratio of $\mathbf{4 g}$ is $50: 50$. $\mathbf{4 g}$ ' was not isolated from the mixture. $\mathbf{4 g}$ were subjected to TBAF and the free diols was isolated via flash chromatography on silica as a colorless oil.

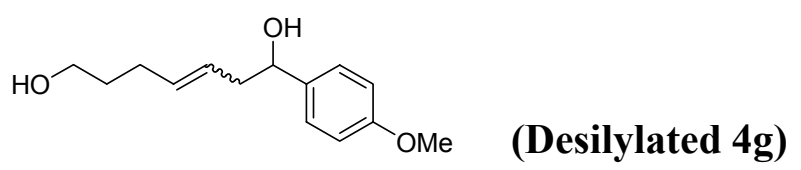

${ }^{1} \mathrm{H}$ NMR (400 MHz, $\left.\mathrm{CDCl}_{3}, \delta\right): 7.26-7.28$ (m, 2H); 6.89 (d, $\left.J=8.6 \mathrm{~Hz}, 2 \mathrm{H}\right) ; 5.41-5.63$ (m, 2H), $4.70(\mathrm{dd}, J=4.8,8.0 \mathrm{~Hz}, 0.5 \mathrm{H}), 4.64(\mathrm{dd}, J=7.2,7.2 \mathrm{~Hz}, 0.5 \mathrm{H}), 3.81(\mathrm{~s}, 3 \mathrm{H}), 3.60-3.65$ (m, 2H), 2.39-2.62 (m, 2H), 2.10-2.27 (m, 2H), 1.89 (brs, 2H), 1.58-1.67 (m, 2H).

${ }^{13} \mathrm{C}$ NMR (100 MHz, $\left.\mathrm{CDCl}_{3}, \delta\right): 159.2,159.2,136.5,136.4,134.2,132.5,127.2,127.2,126.6$, 126.1, 114.0, 113.9, 73.7, 73.4, 62.7, 62.0, 55.5, 42.8, 37.3, 32.3, 32.1, 29.5, 23.7.

IR (NaCl, thin film): 3354, 2933, 1612, 1513, 1442, 1303, 1247, 1175, 1035, 832.

HRMS-ESI (m/z): [M+Na] $]^{+}$calcd for $\mathrm{C}_{26} \mathrm{H}_{48} \mathrm{O}_{3} \mathrm{Si}_{2} \mathrm{Na}$, 487.3040; found, 487.3017. 


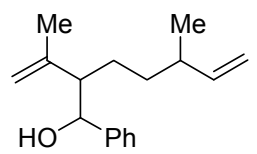

(5a)

To $\beta$-Citronellene $(0.5 \mathrm{mmol})$ in anhydrous $\mathrm{CH}_{2} \mathrm{Cl}_{2}(5 \mathrm{~mL})$ was added $\mathrm{Me}_{2} \mathrm{AlCl}(1.0 \mathrm{M}$ in hexane, $1.1 \mathrm{~mL}$ ) at $0{ }^{\circ} \mathrm{C}$. The mixture was stirred at room temperature for $24 \mathrm{~h}$. The reaction was quenched by diluting the reaction mixture with diethylether, followed by slow addition of water until gas evolution ceased. The organic layer was separated, and the aqueous layer was extracted with ether twice. The combined organic layers were washed with brine, dried and evaporated in vacuo. Purification via flash chromatography on silica gel afforded the coupling product $\mathbf{5 a}$ as a colorless oil. Homoallylic alcohol $\mathbf{5 b}$ was not detected.

${ }^{1} \mathrm{H}$ NMR (400 MHz, $\left.\mathrm{CDCl}_{3}, \delta\right): 7.28-7.39(\mathrm{~m}, 5 \mathrm{H}) ; 5.45-5.61(\mathrm{~m}, 1 \mathrm{H}) ; 5.09(\mathrm{~s}, 1 \mathrm{H}) ; 5.00(\mathrm{~s}, 1 \mathrm{H})$; 4.79-4.94 (m, 2H); $4.38(\mathrm{dd}, J=0.7,8.5 \mathrm{~Hz}, 1 \mathrm{H}) ; 2.19-2.35(\mathrm{~m}, 1 \mathrm{H}) ; 1.89-2.05(\mathrm{~m}, 1 \mathrm{H}) ; 1.73$, 1.75 (two s, 3H); 1.67 (brs, $1 \mathrm{H}) ; 0.91-1.27$ (m, 4H); 0.87, 0.84 (two d, $J=6.8 \mathrm{~Hz}, 3 \mathrm{H}$ ).

${ }^{13} \mathrm{C}$ NMR (100 MHz, $\left.\mathrm{CDCl}_{3}, \delta\right): 145.1,144.3,142.9,128.5,127.9,127.4,127.3,126.0,116.5$, $116.4,113.1,112.4,75.5,75.4,56.5,56.4,37.8,37.5,34.2,34.2,31.2,26.3,21.0,19.5,18.3$, 18.1.

HRMS-EI (m / z): [M] ${ }^{+*}$ calcd for $\mathrm{C}_{17} \mathrm{H}_{24} \mathrm{ONa}, 244.182$; found, 244.182.

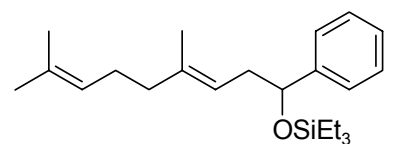

(5b)

The reaction of $\beta$-Citronellene and benzaldehyde $(51 \mu \mathrm{L}, 0.5 \mathrm{mmol}, 100 \mathrm{~mol} \%)$ with $\mathrm{Ni}(\operatorname{cod})_{2}$, $\mathrm{EtOPh}_{2} \mathrm{P}(43 \mu \mathrm{l}, 0.2 \mathrm{mmol}, 40 \mathrm{~mol} \%)$ and TESOTf $(197 \mu \mathrm{L}, 0.875 \mathrm{mmol}, 175 \mathrm{~mol} \%)$, triethylamine in toluene following the general procedure 3 above afforded $\mathbf{5 b} \mathbf{7 5 \%}$ total yield according to ${ }^{1} \mathrm{H}$ NMR of the crude mixture and the E/Z ratio of $\mathbf{5 b}$ is 71:29. 5a was not detected. Purification via flash chromatography on silica afforded $\mathbf{5 b}$ as a colorless oil.

${ }^{1} \mathrm{H}$ NMR (400 MHz, $\left.\mathrm{CDCl}_{3}, \delta\right): 7.25-7.36(\mathrm{~m}, 5 \mathrm{H}) ; 5.18-5.20(\mathrm{~m}, 1 \mathrm{H}) ; 5.12-5.12(\mathrm{~m}, 1 \mathrm{H})$; 4.64-4.68 (m, 1H); 2.37-2.48 (m, 2H); 2.01-2.10 (m, 4H); $1.73(\mathrm{~m}, 4 \mathrm{H}) ; 1.64(\mathrm{~m}, 3 \mathrm{H}) ; 1.55$ (s, $2 \mathrm{H}) ; 0.92(\mathrm{t}, J=6.9 \mathrm{~Hz}, 9 \mathrm{H}) ; 0.57(\mathrm{dq}, J=3.0,6.9 \mathrm{~Hz}, 6 \mathrm{H})$.

${ }^{13} \mathrm{C}$ NMR (100 MHz, $\left.\mathrm{CDCl}_{3}, \delta\right): 145.8,145.8,137.2,137.1,131.7,131.5,128.1,128.0,127.0$, $127.0,126.1,126.1,124.6,124.5,121.8,120.7,75.5,75.3,40.0,39.8,39.7,32.3,26.8,26.7,25.9$, 25.9, 23.6, 17.8, 17.8, 16.3, 7.0, 7.0, 5.0, 5.0.

IR (NaCl, thin film): 2955, 2876, 1454, 1376, 1239, 1088, 1068, 1006, 829, 743, 700. HRMS-ESI (m / z): $[\mathrm{M}+\mathrm{Na}]^{+}$calcd for $\mathrm{C}_{23} \mathrm{H}_{28} \mathrm{OSiNa}$, 381.2590; found, 381.2583. 
$\psi_{2}=$

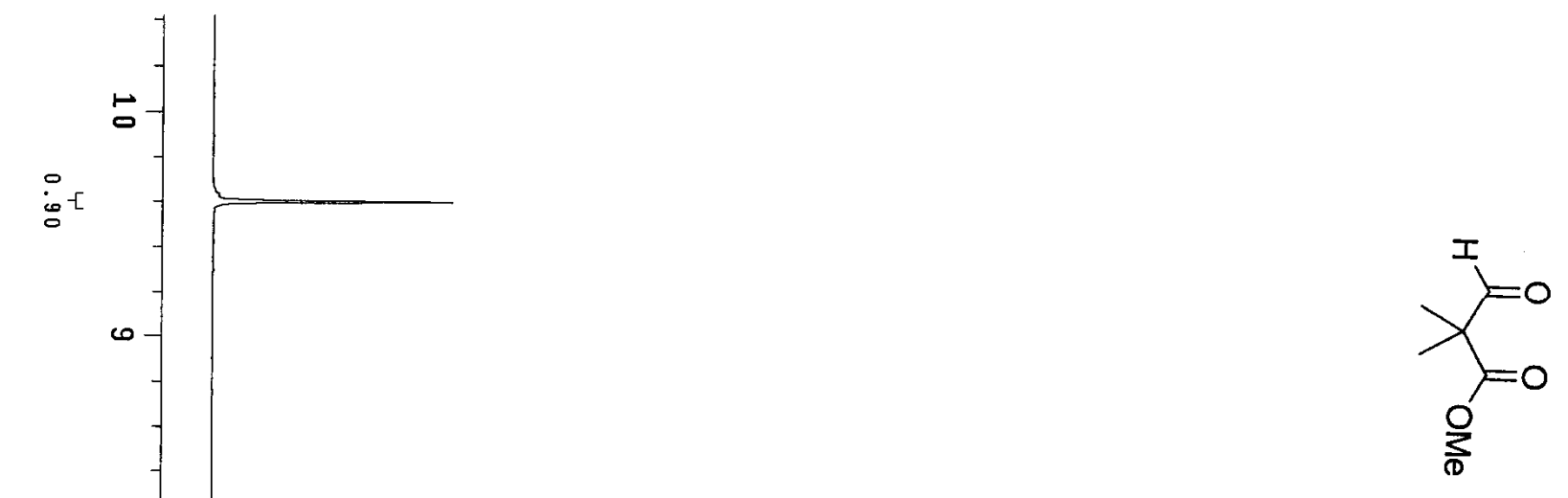

ڤ.

$\omega$

$\infty$
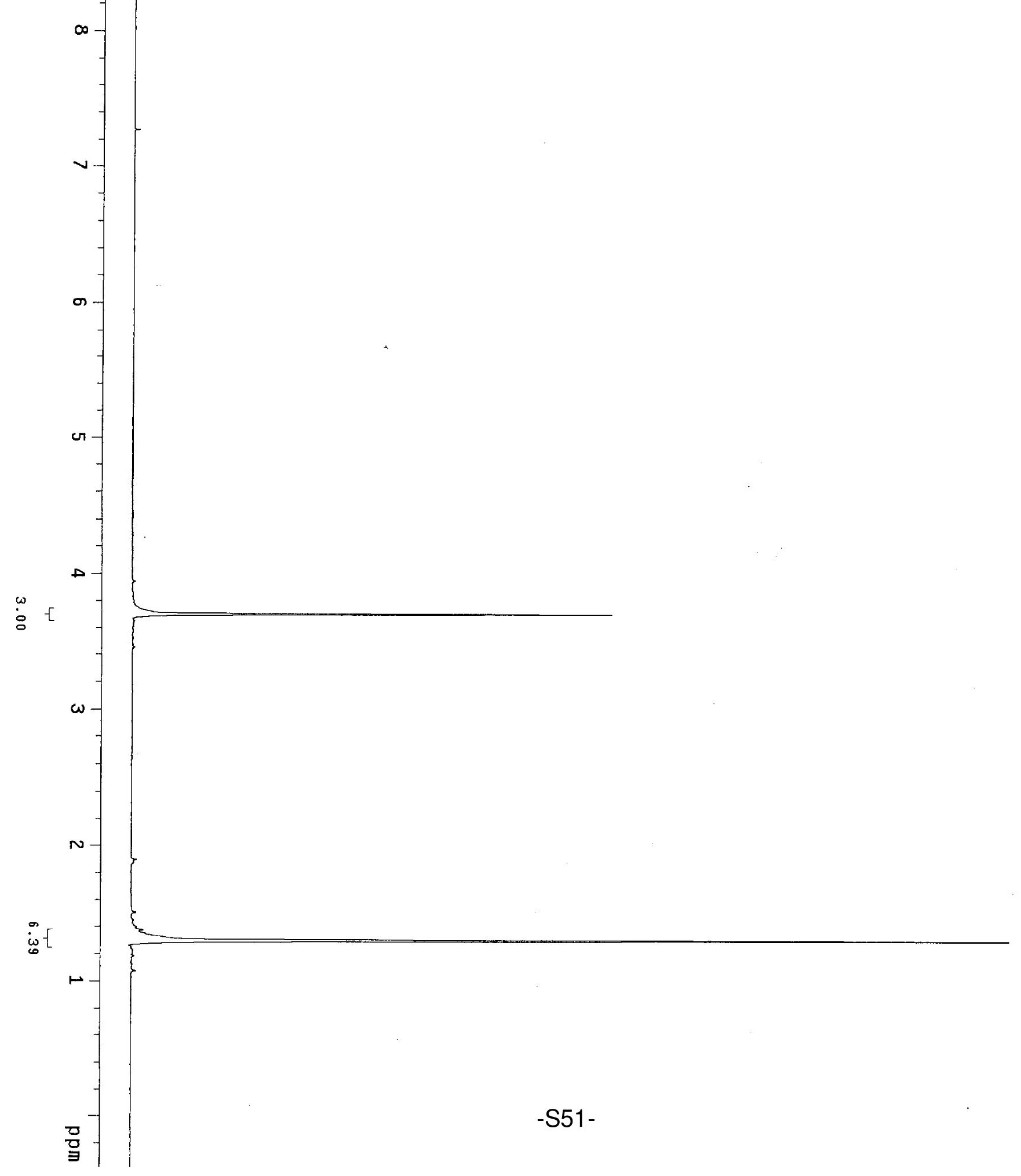

$\omega$

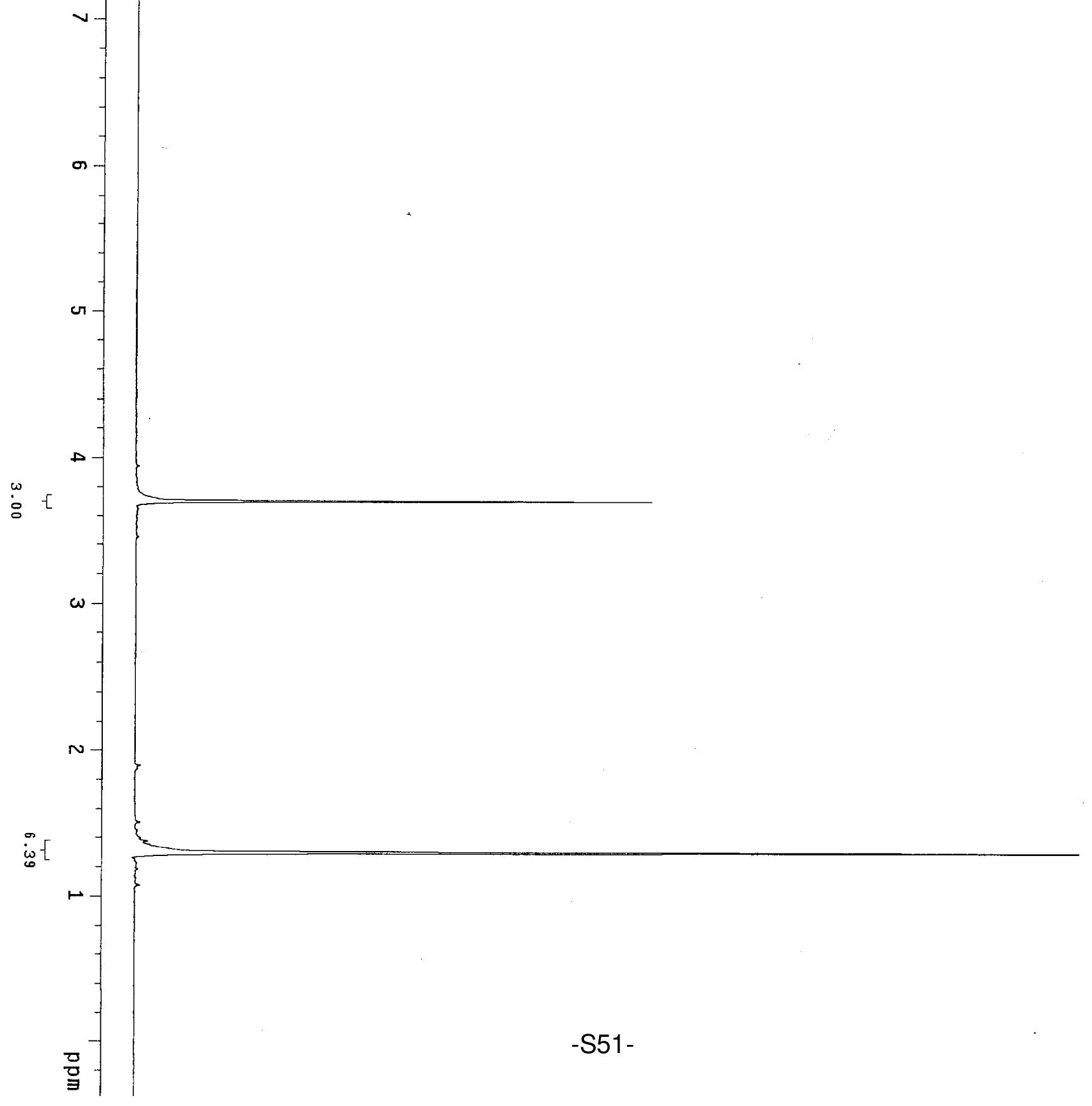


i.

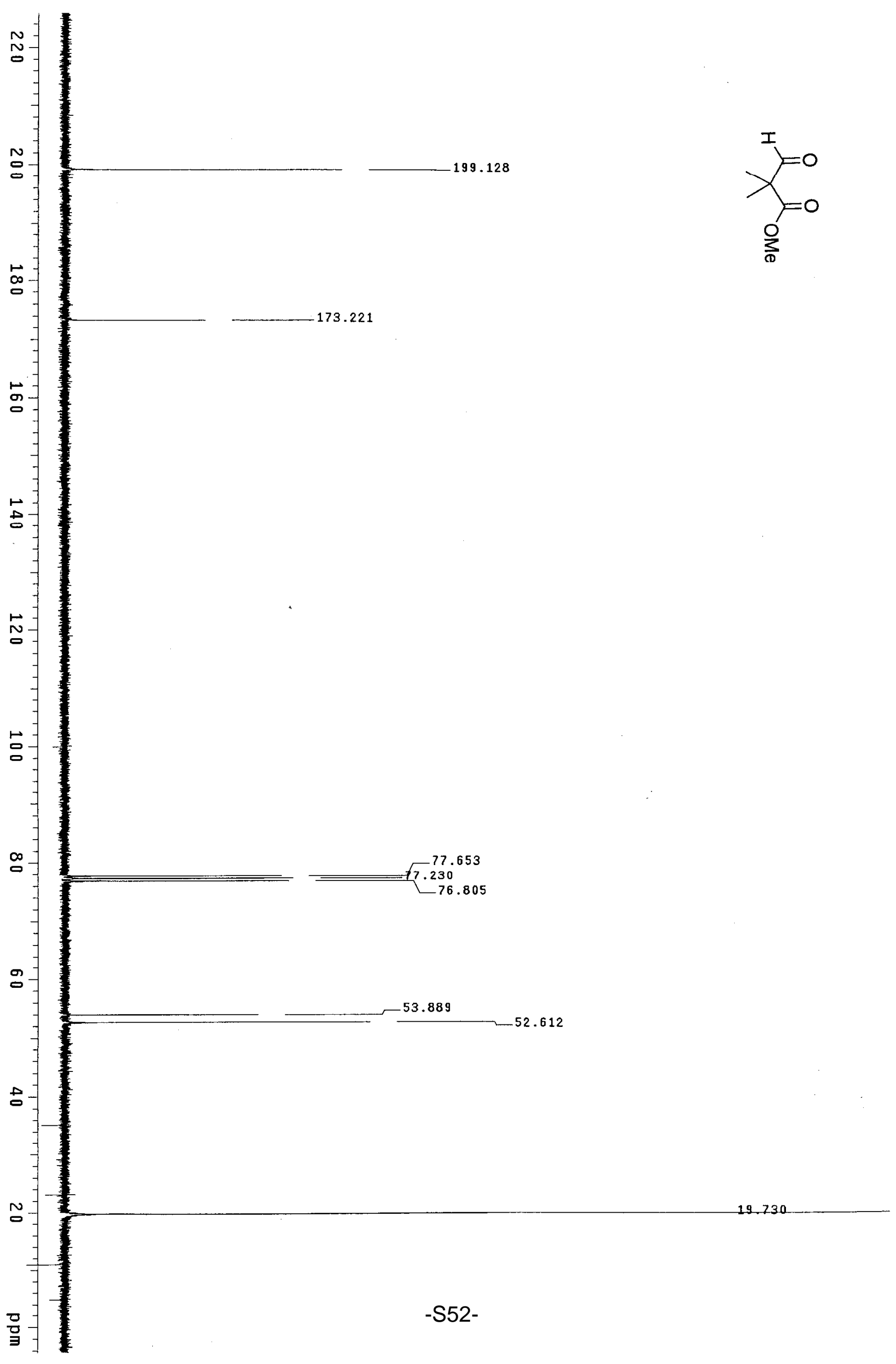



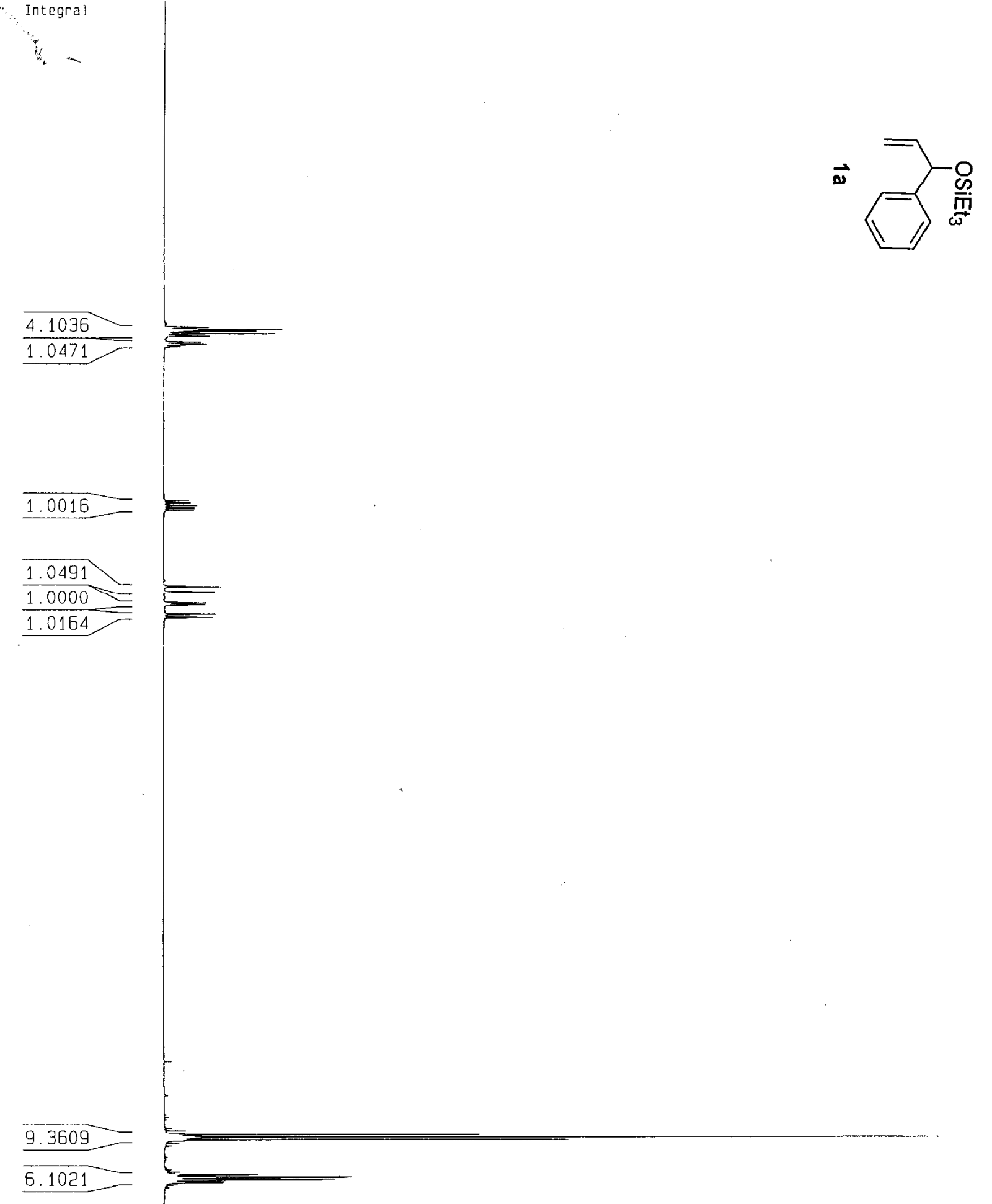

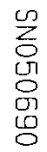

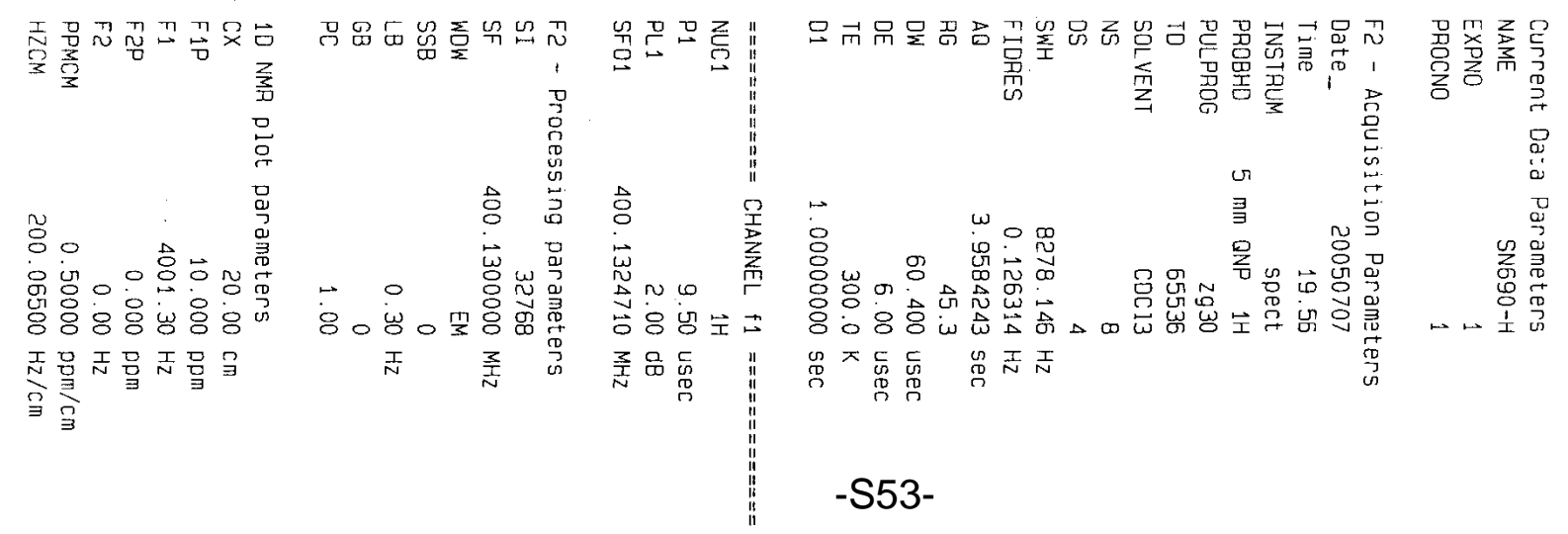




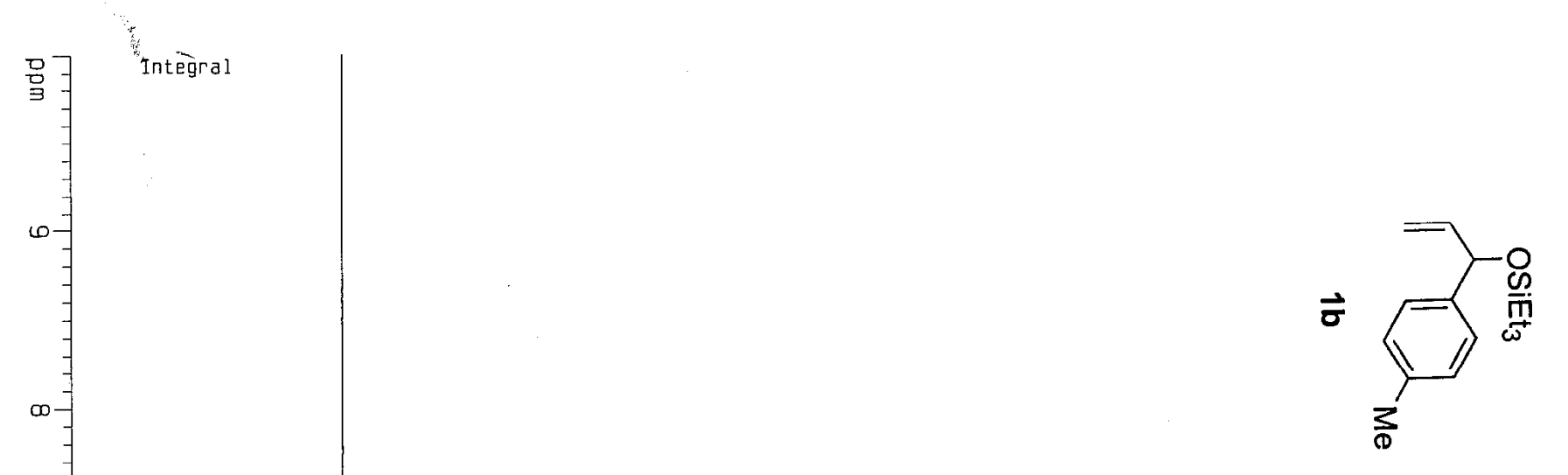



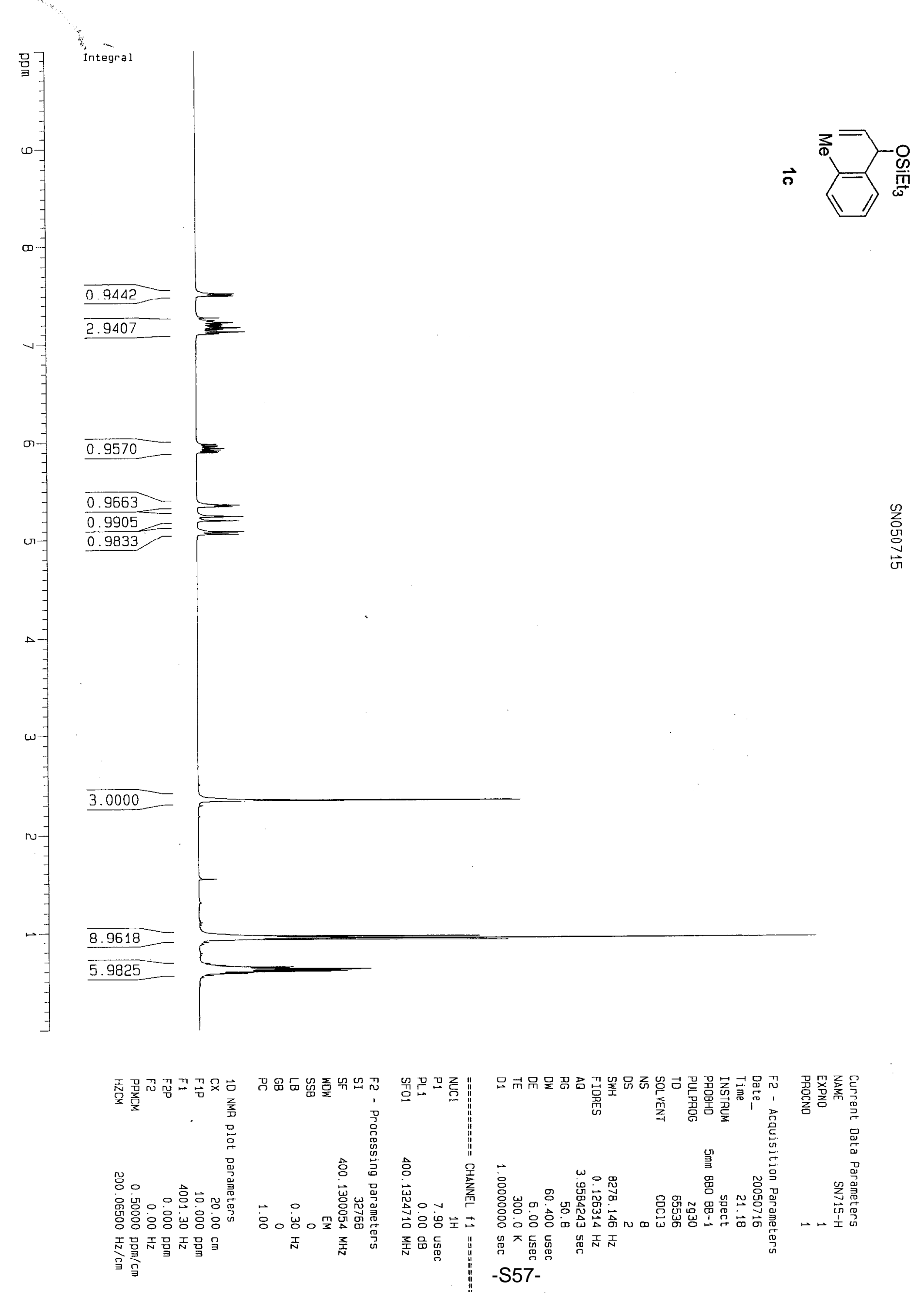


$$
E
$$



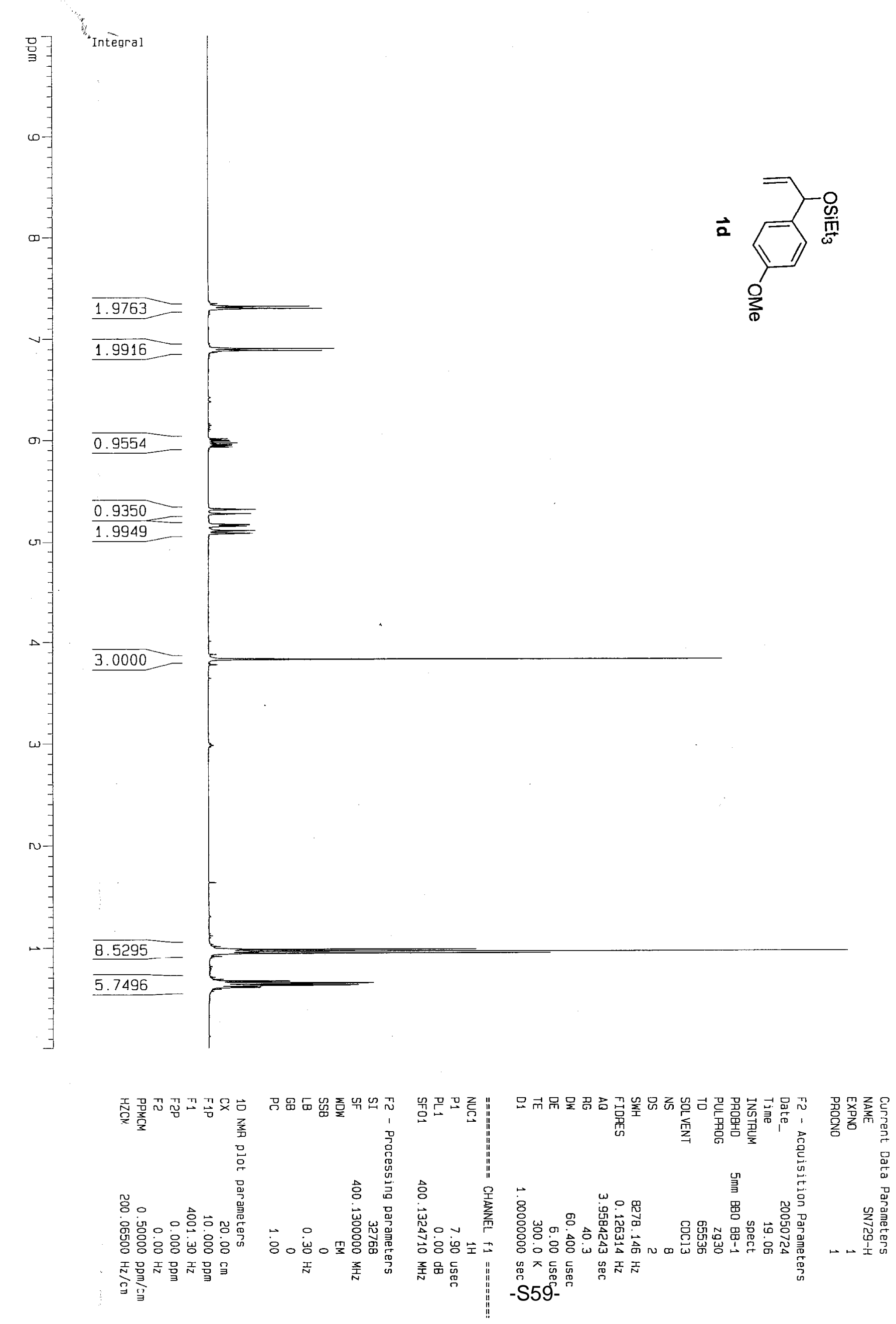


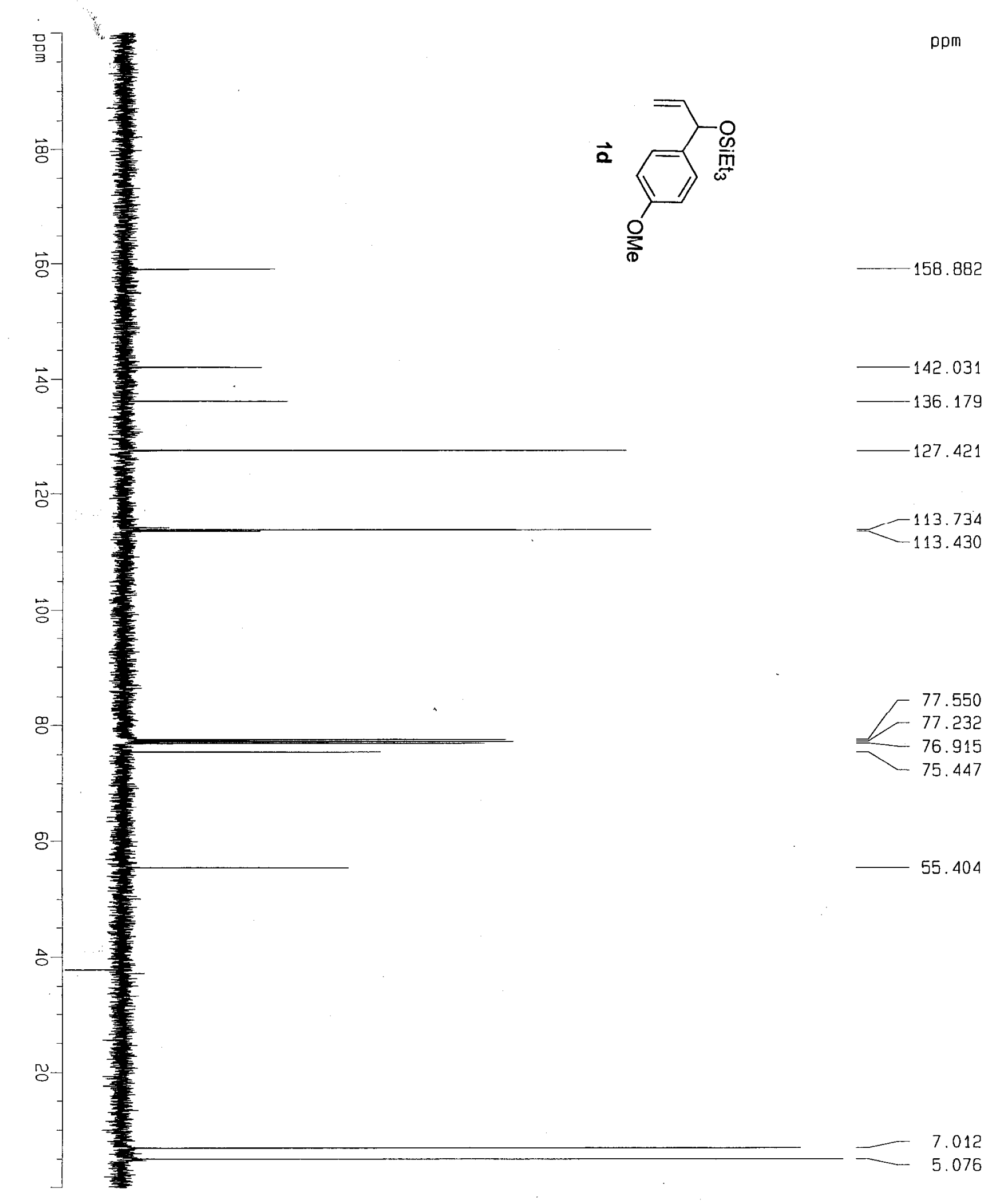

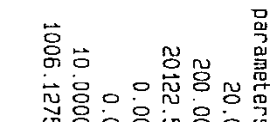

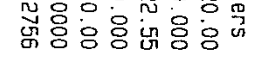

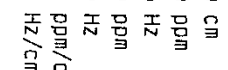

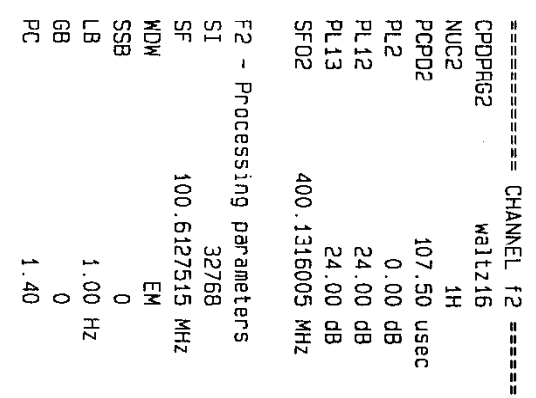
긐

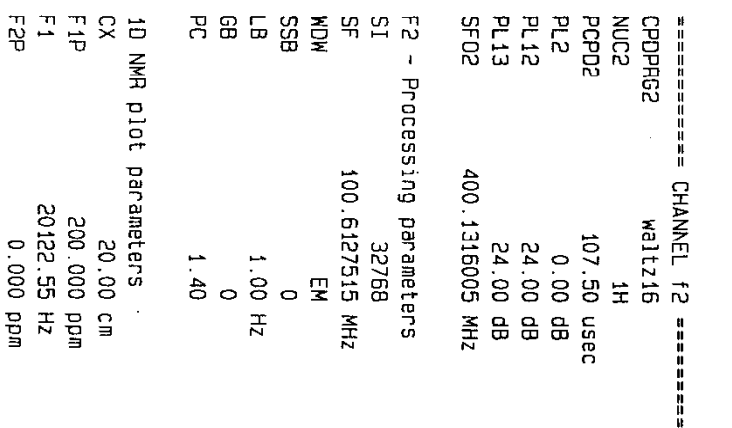

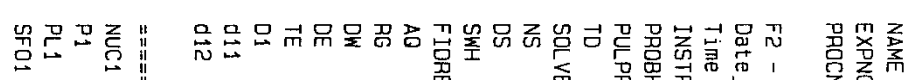

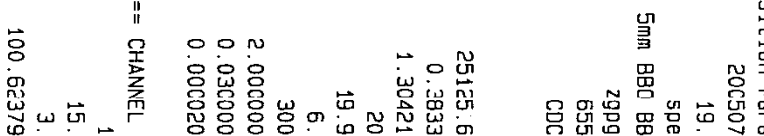

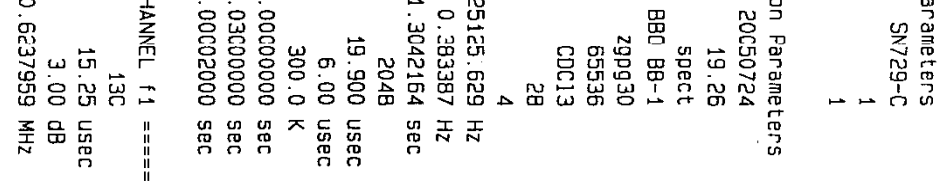
$-\mathrm{S60}-$

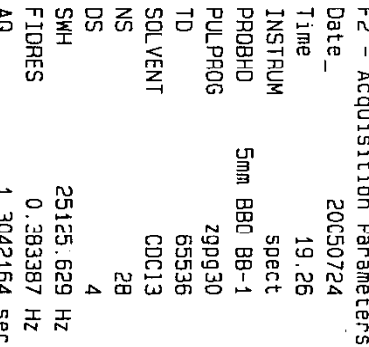

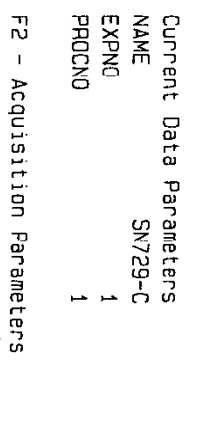




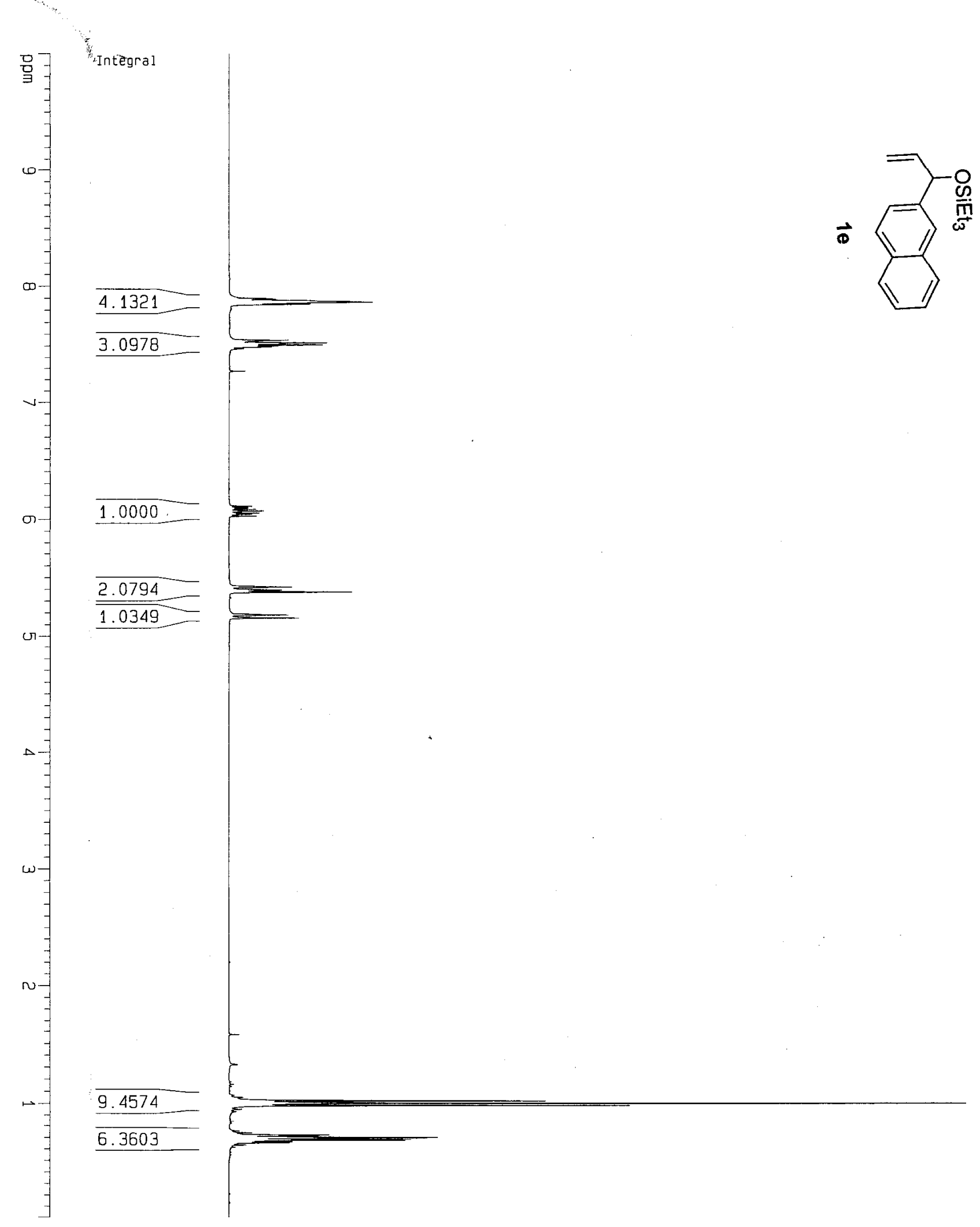

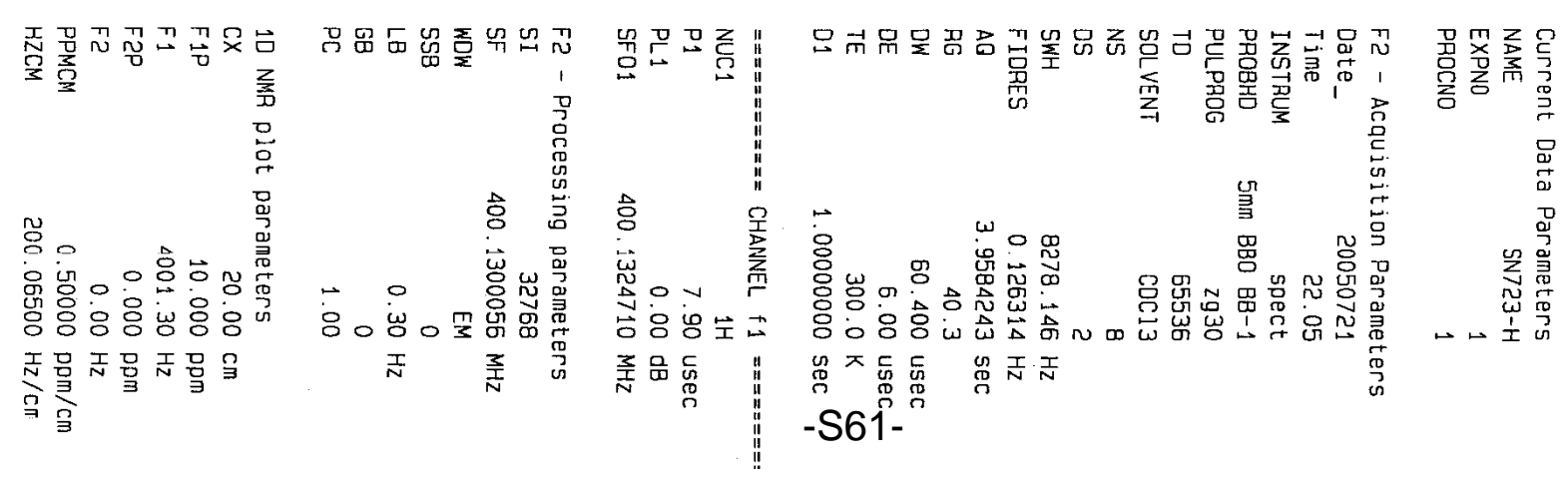




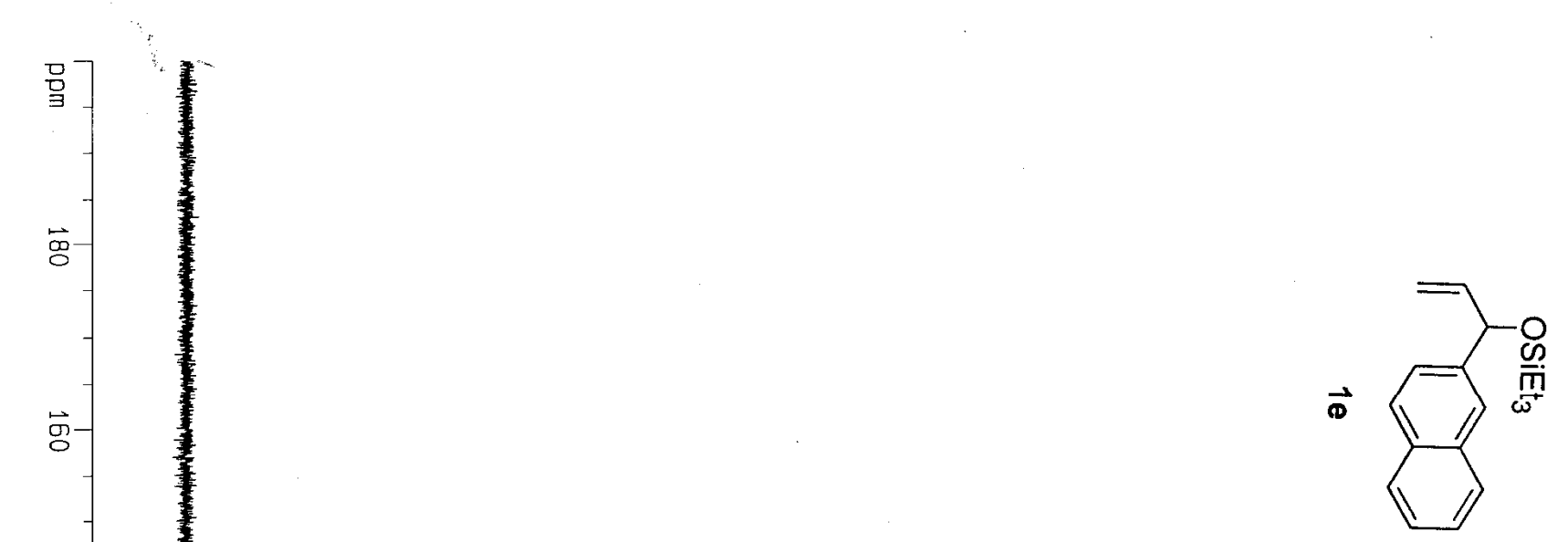

$\sqrt{\text { ( }}$

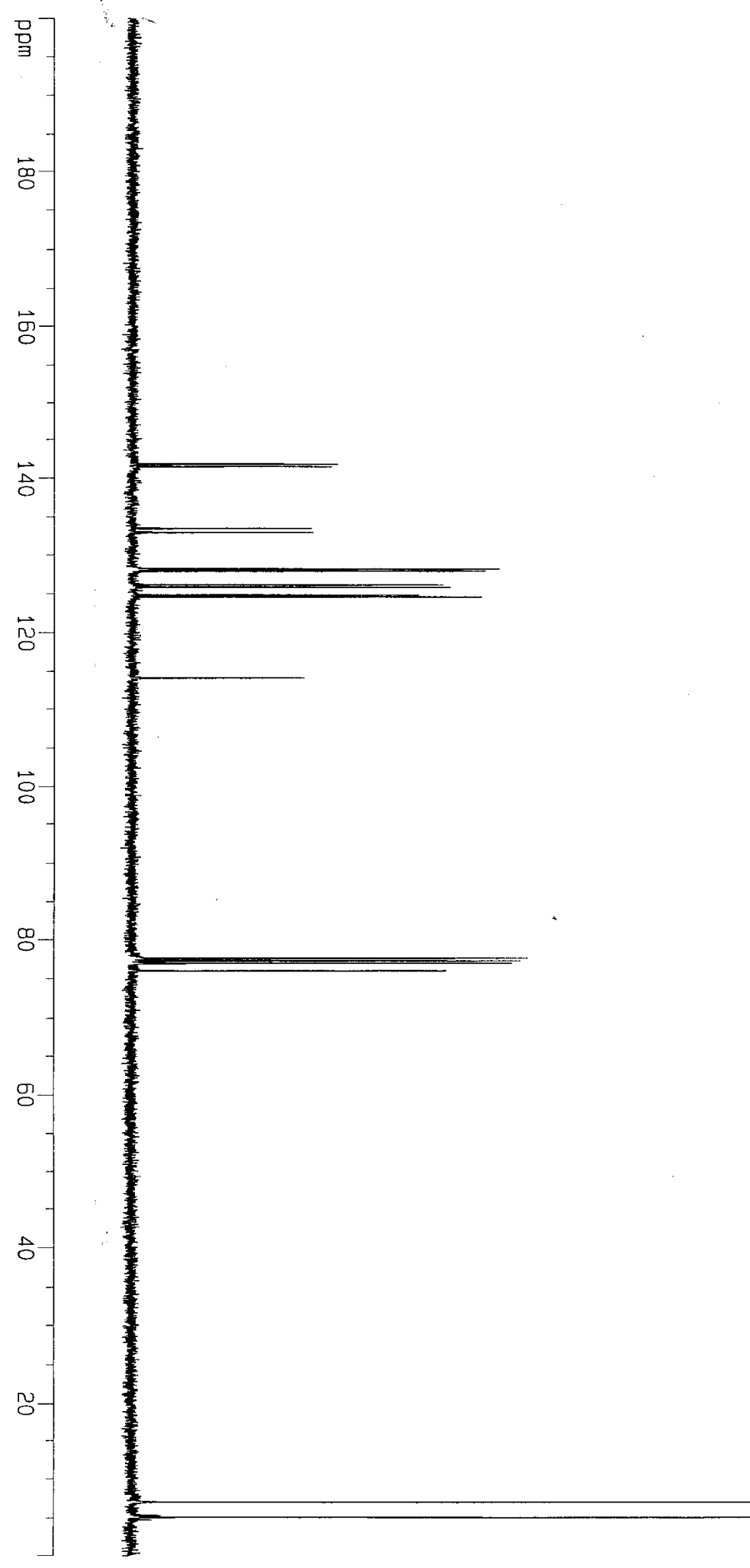

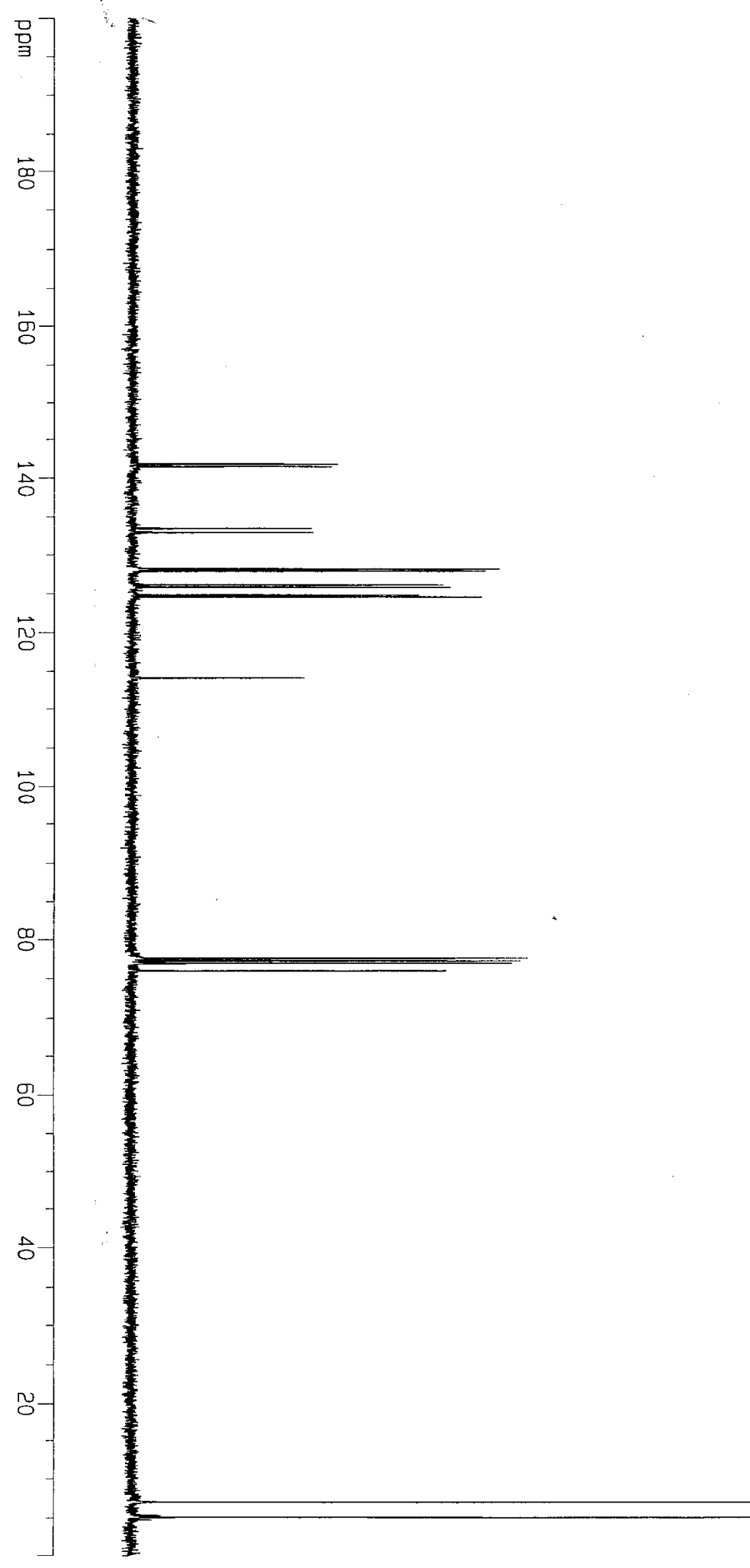

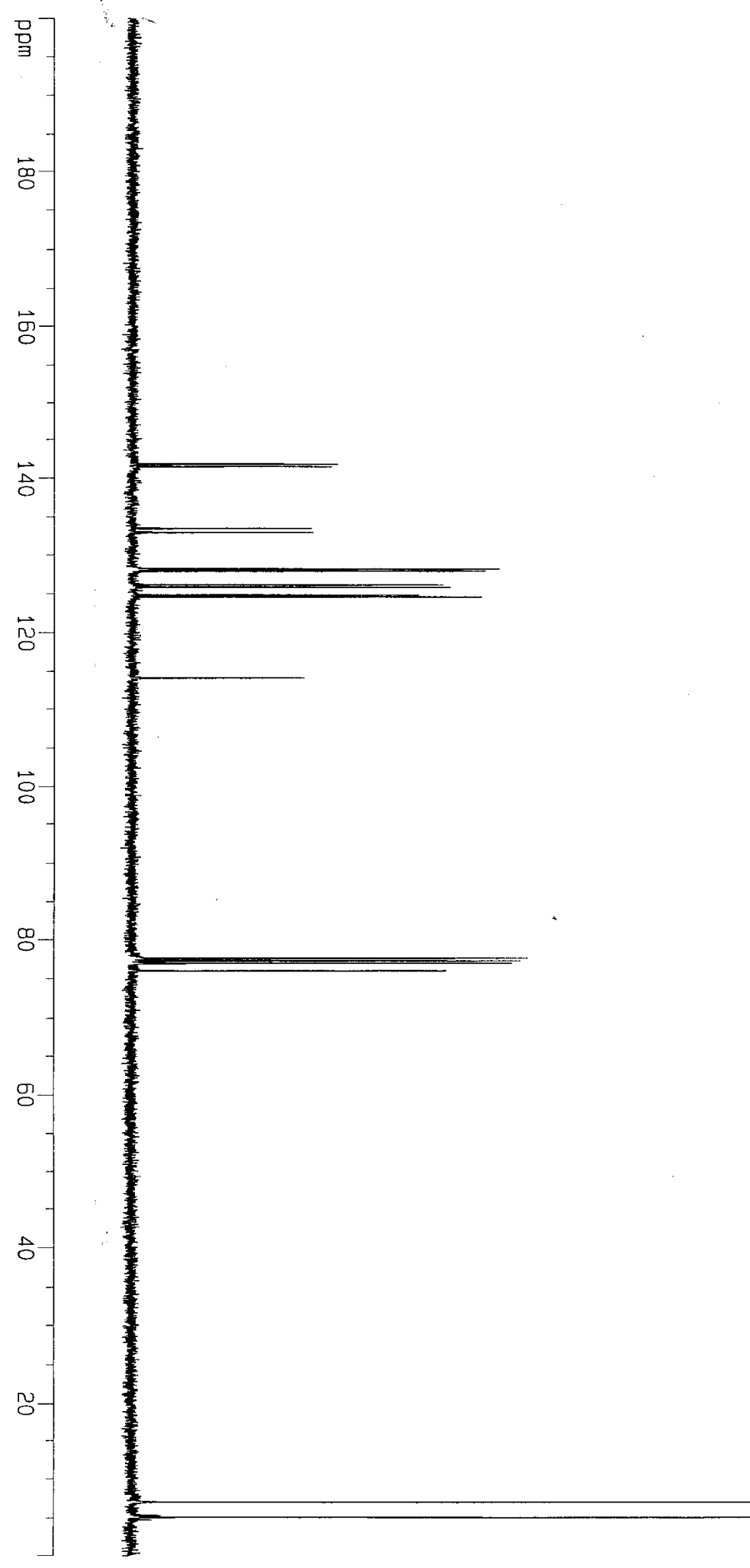

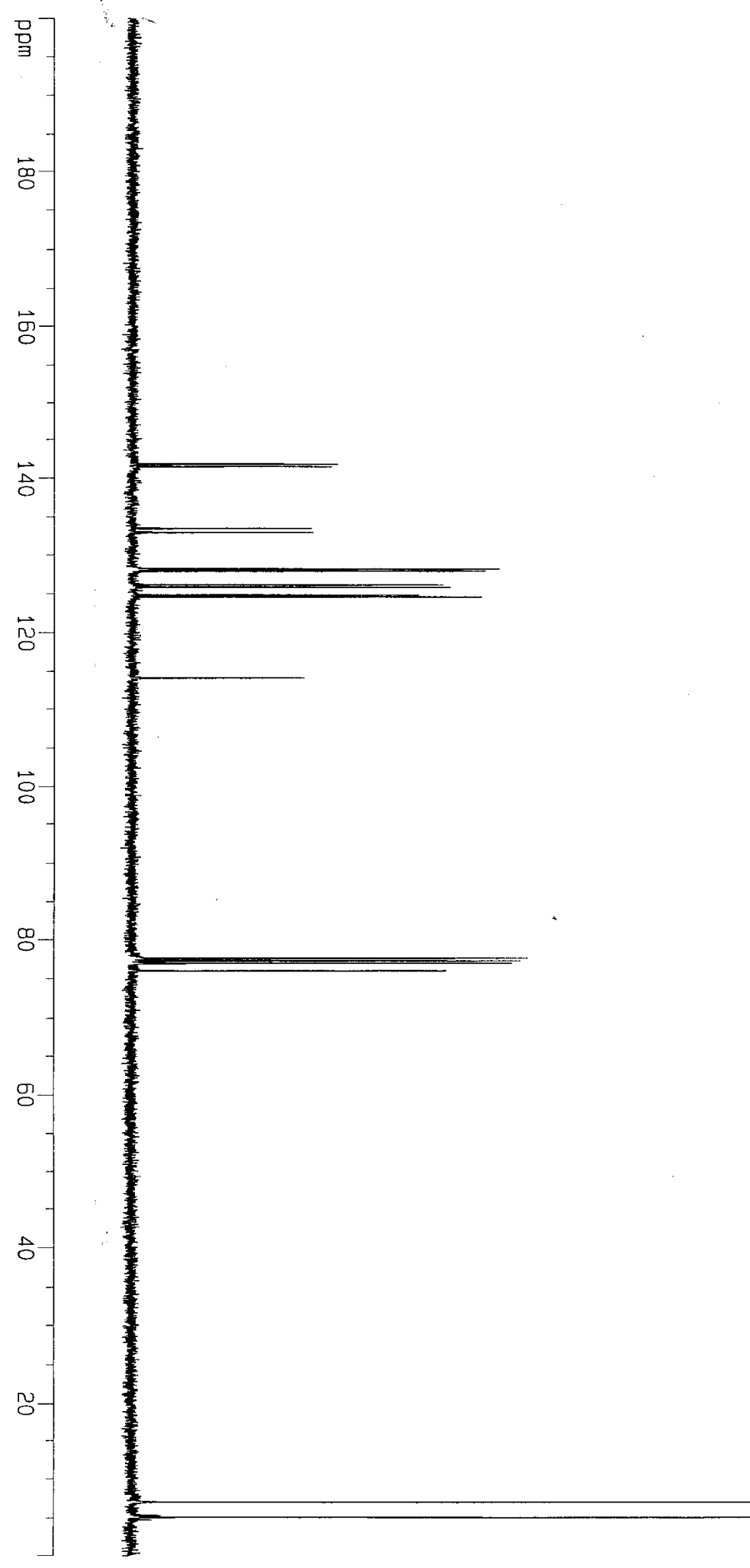

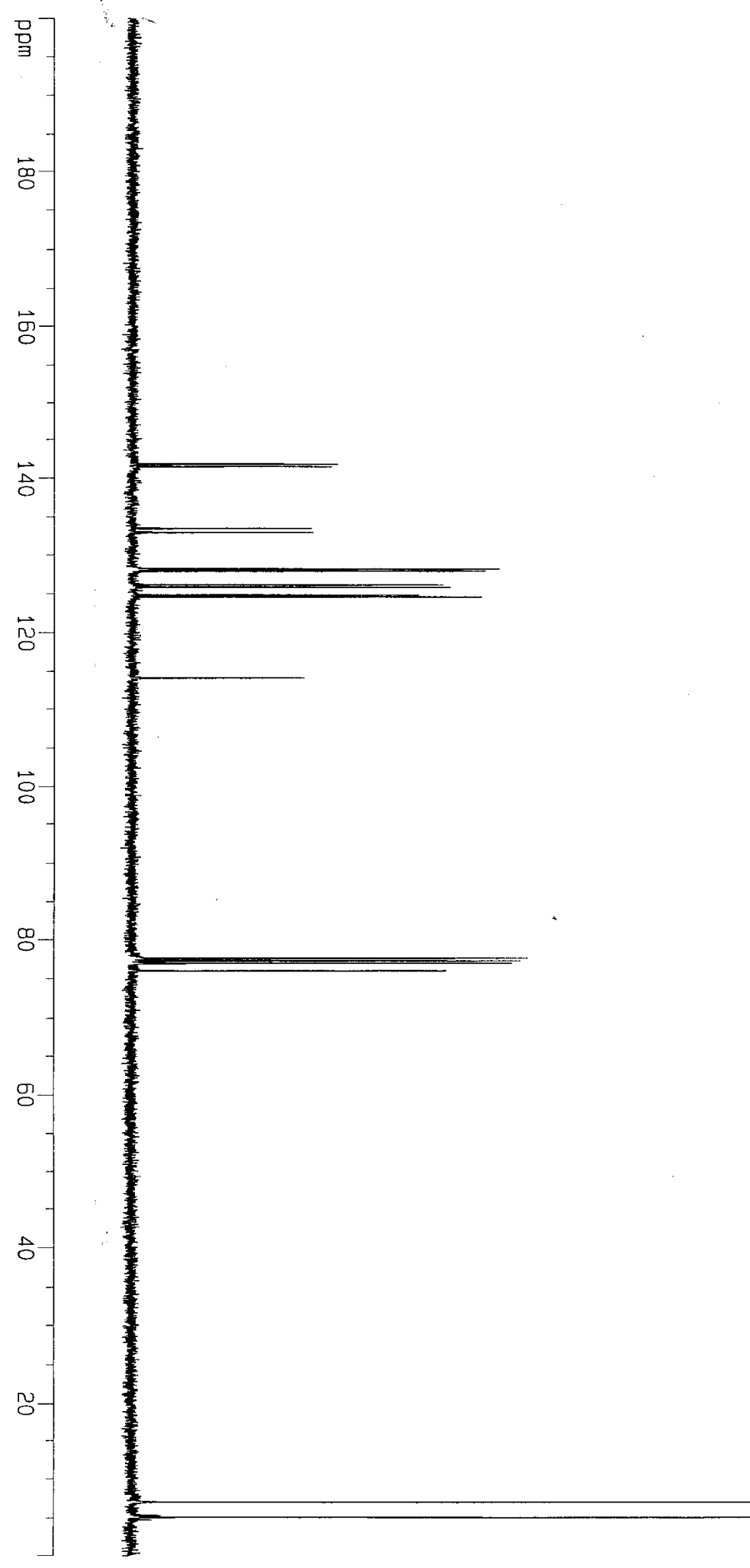

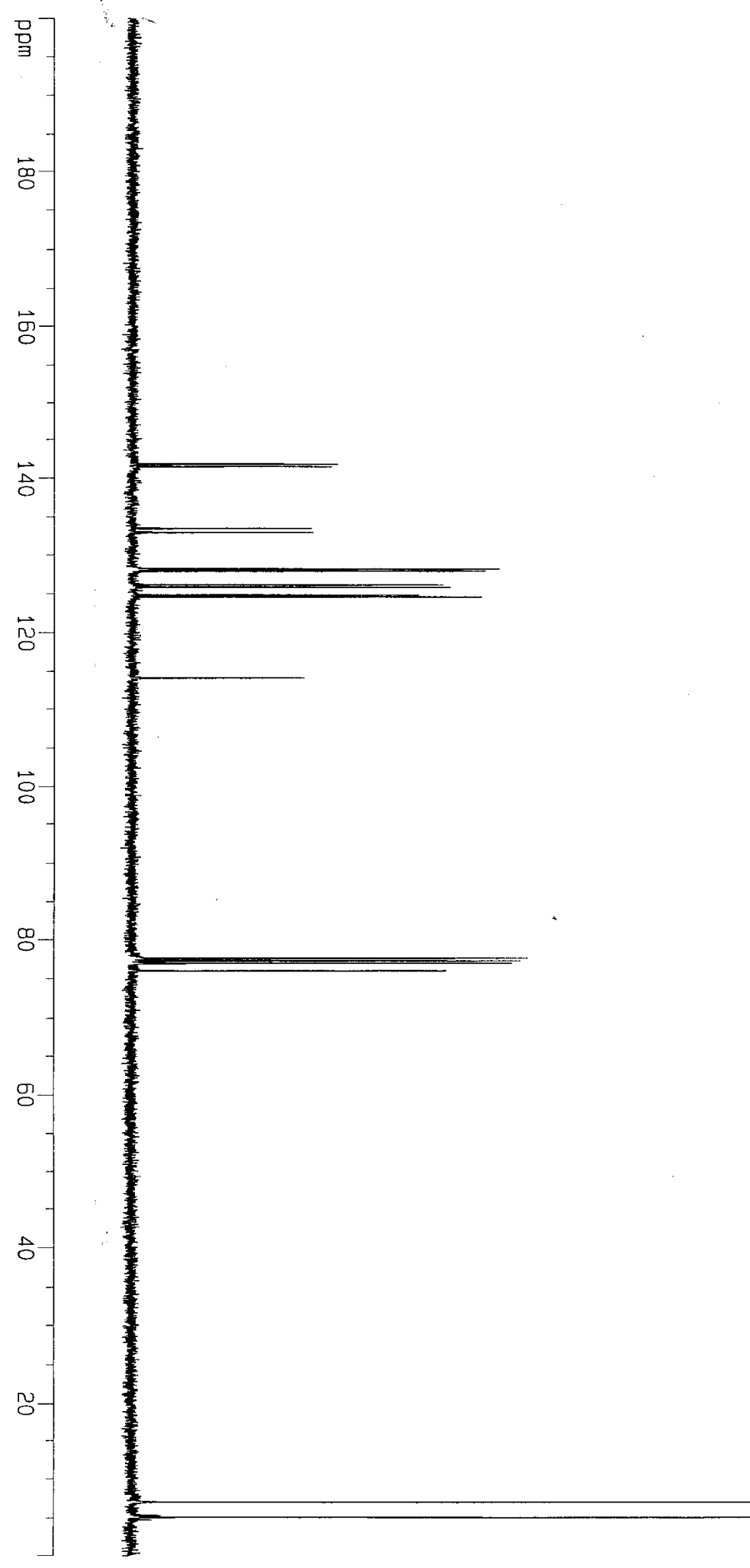

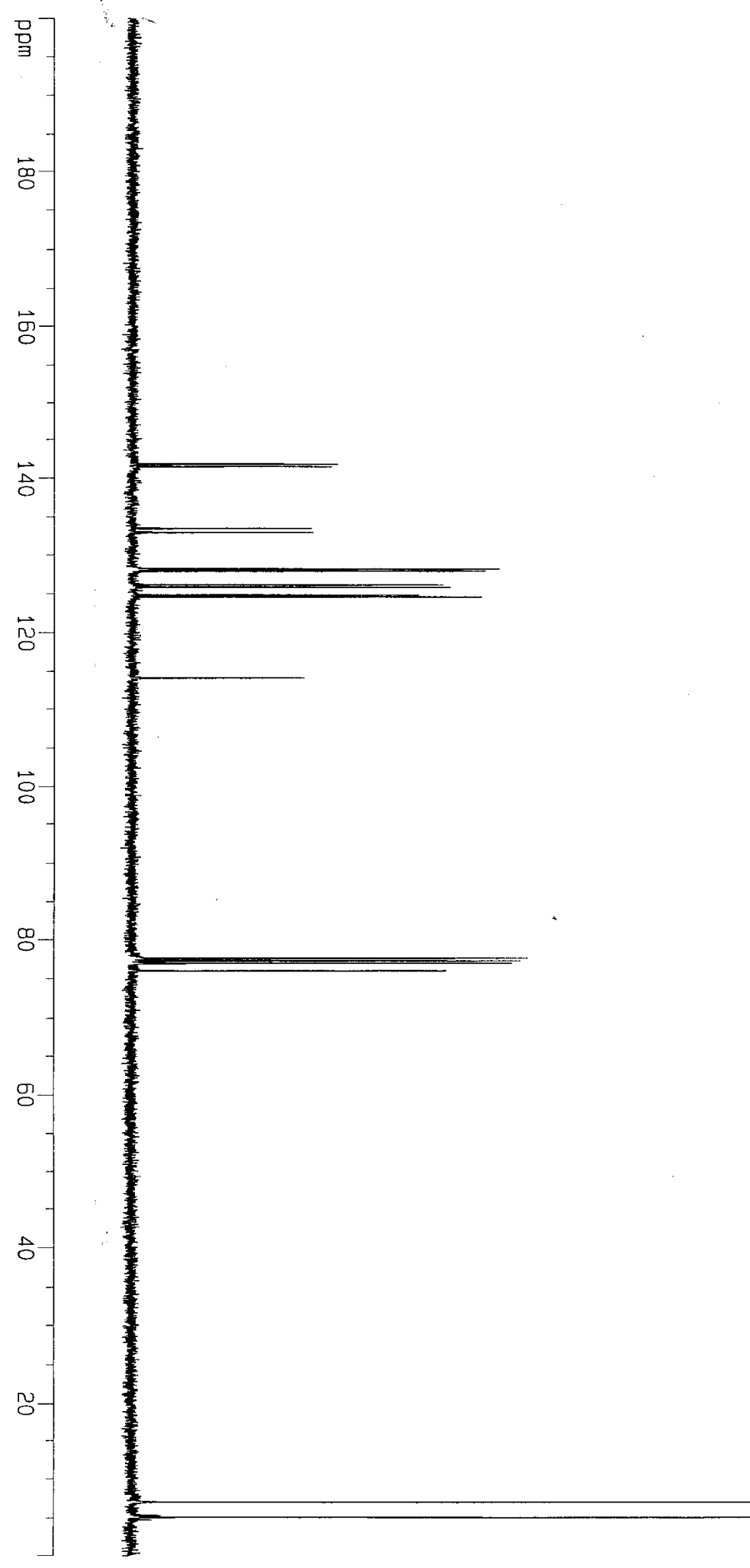

(1)

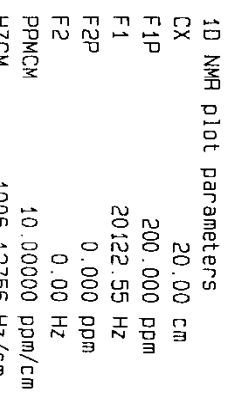

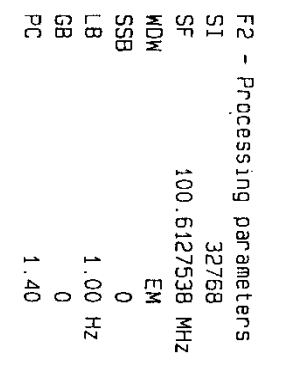

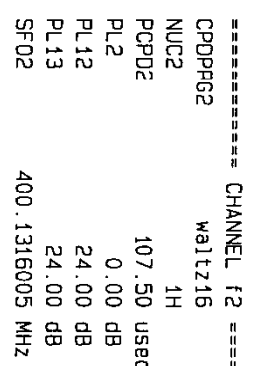

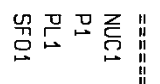

0
Ò
U్
U్ 


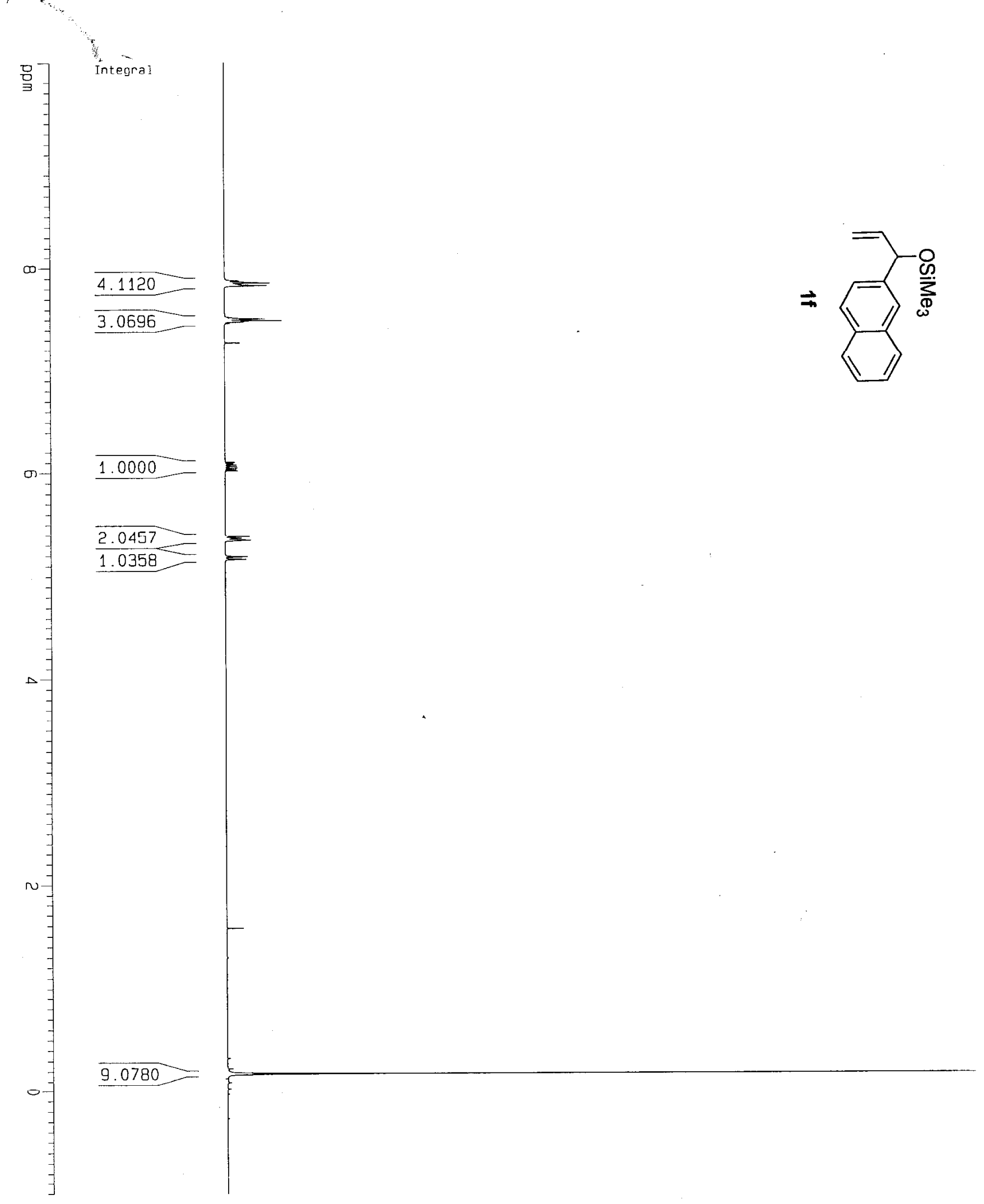

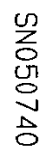

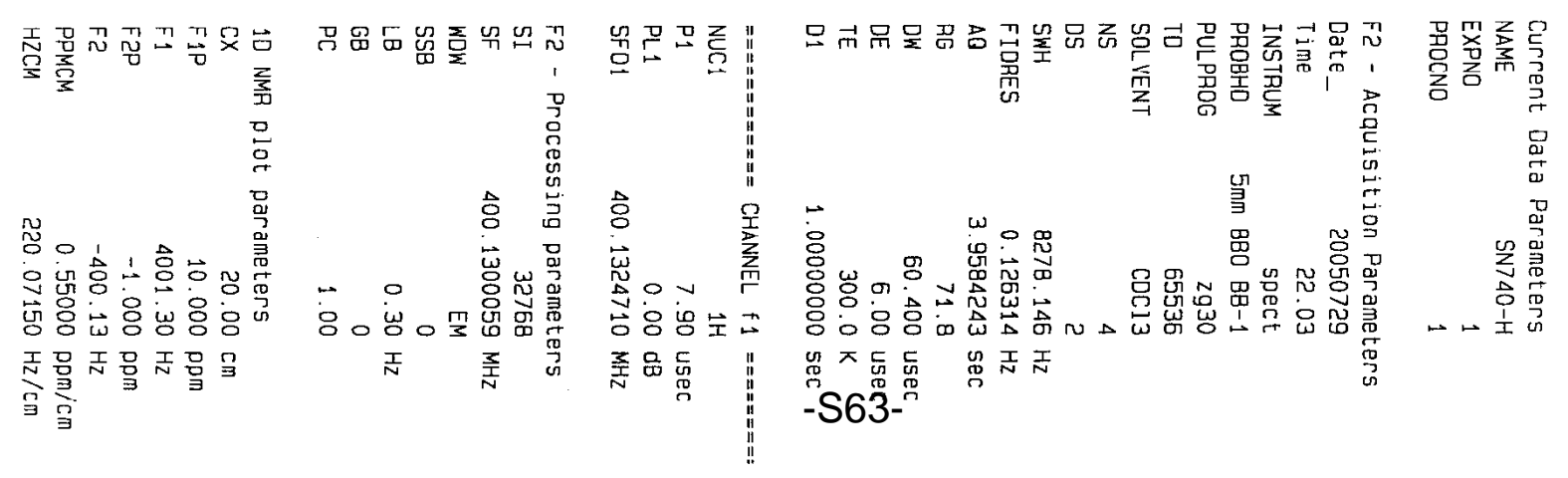




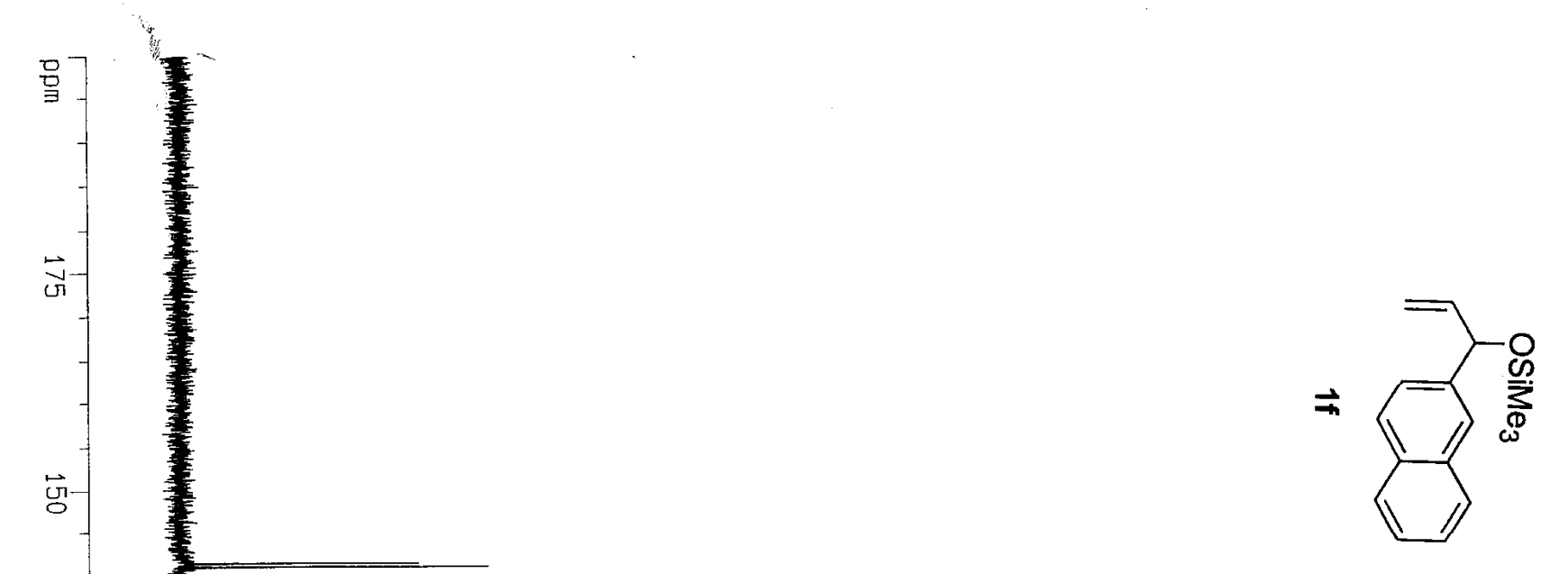

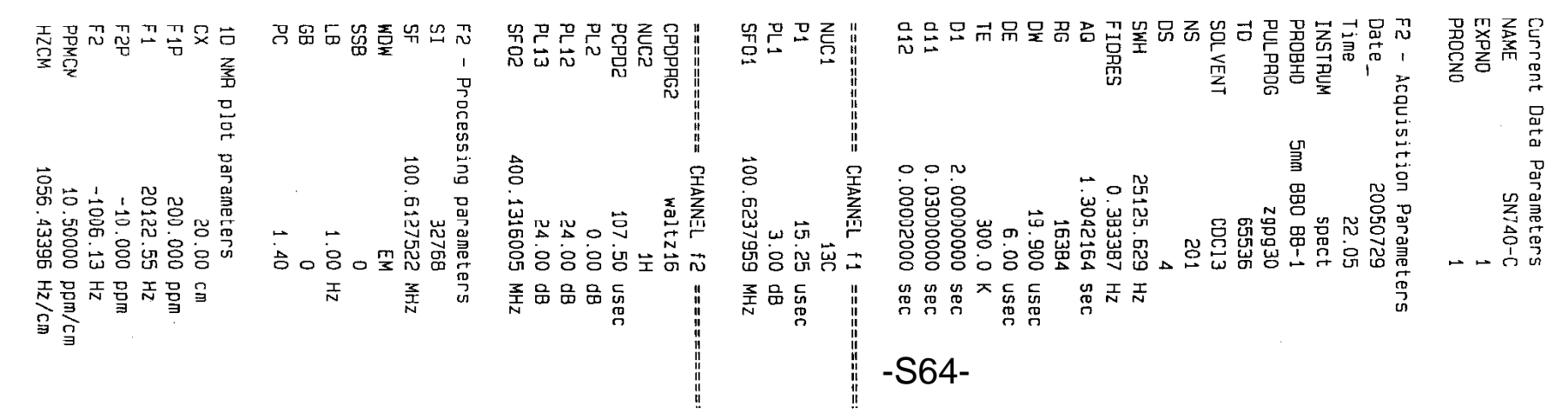



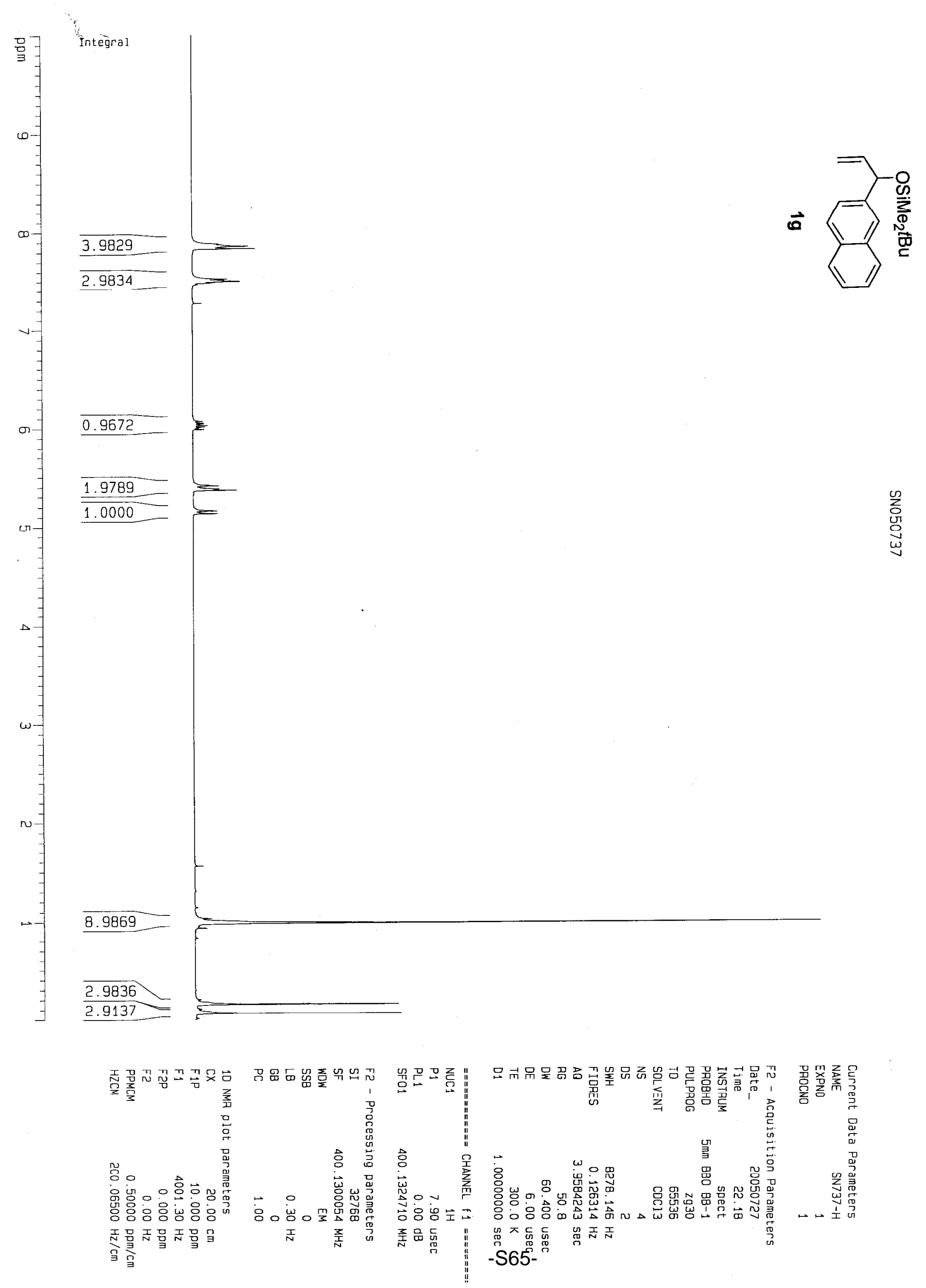


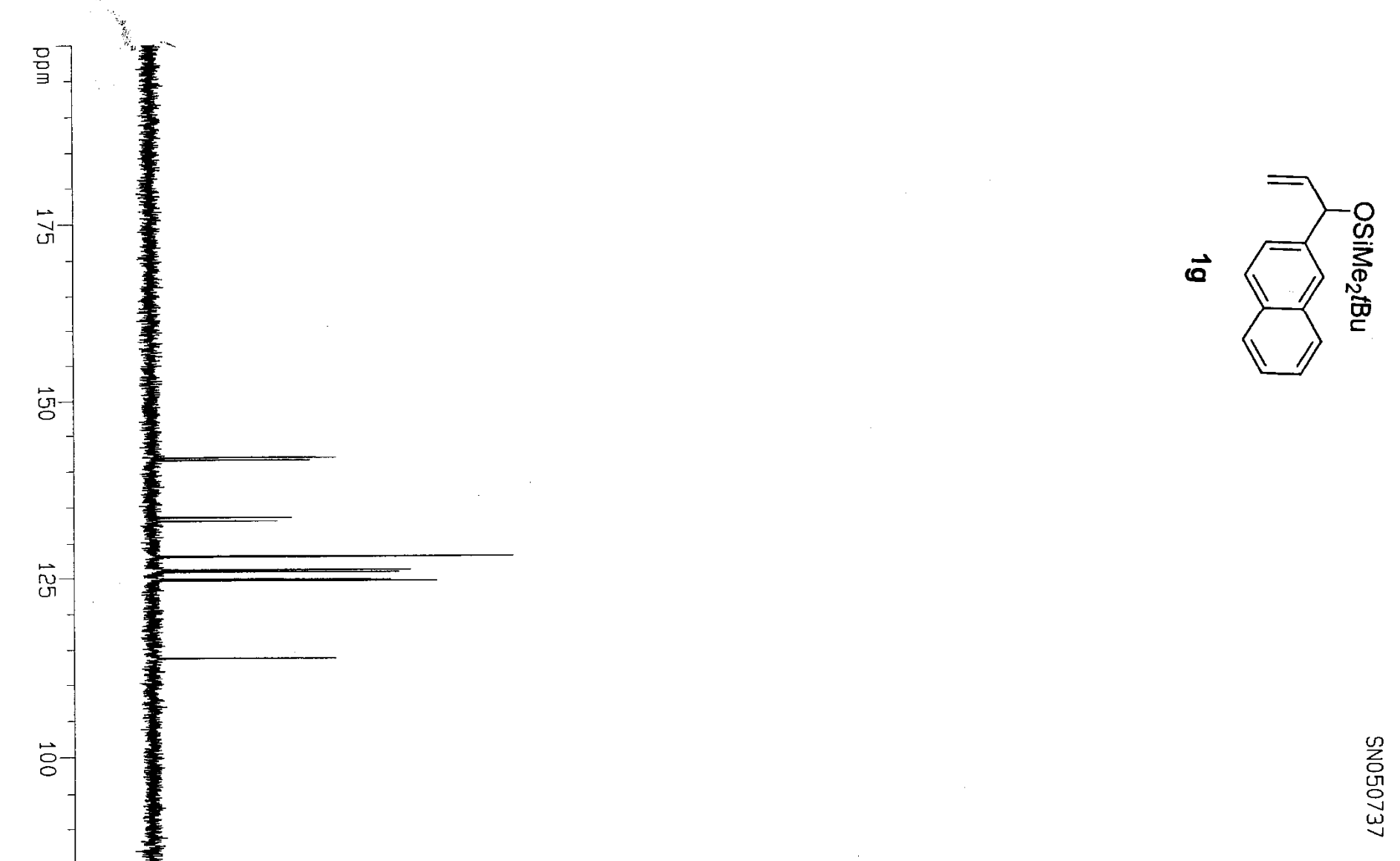

울

$\frac{\sqrt{3}}{3}$

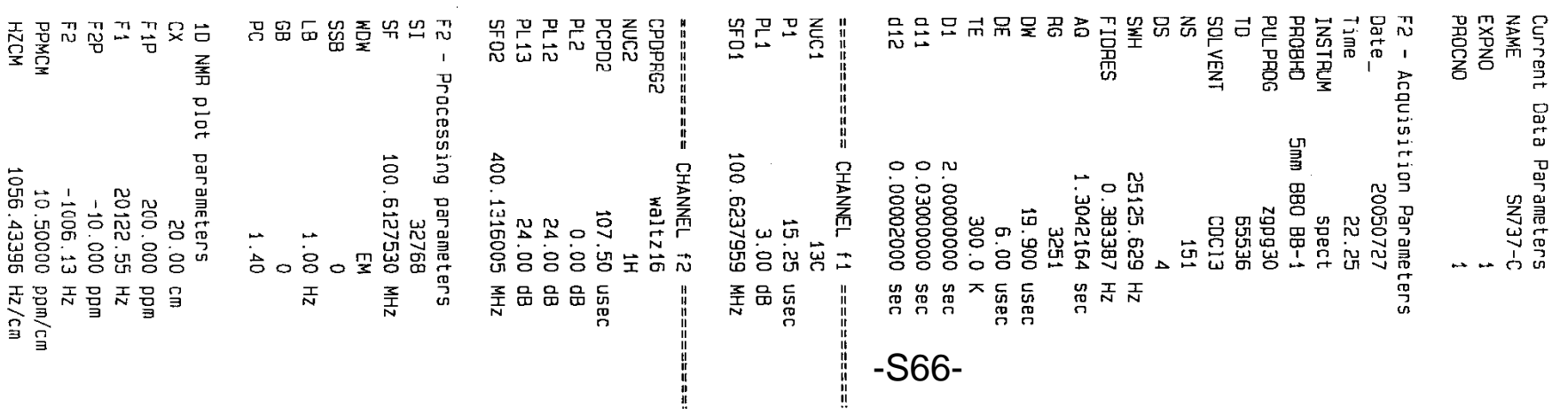




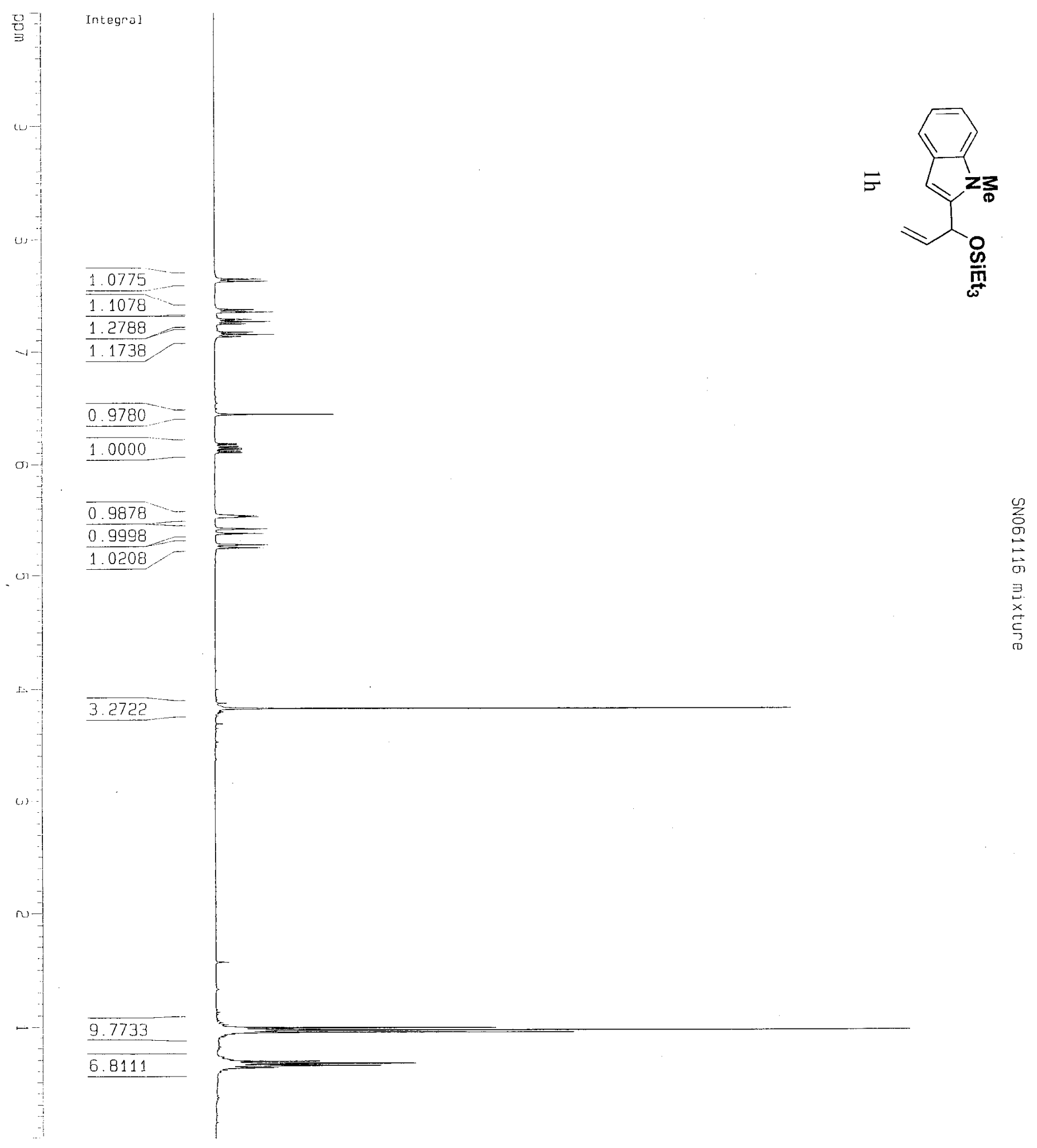

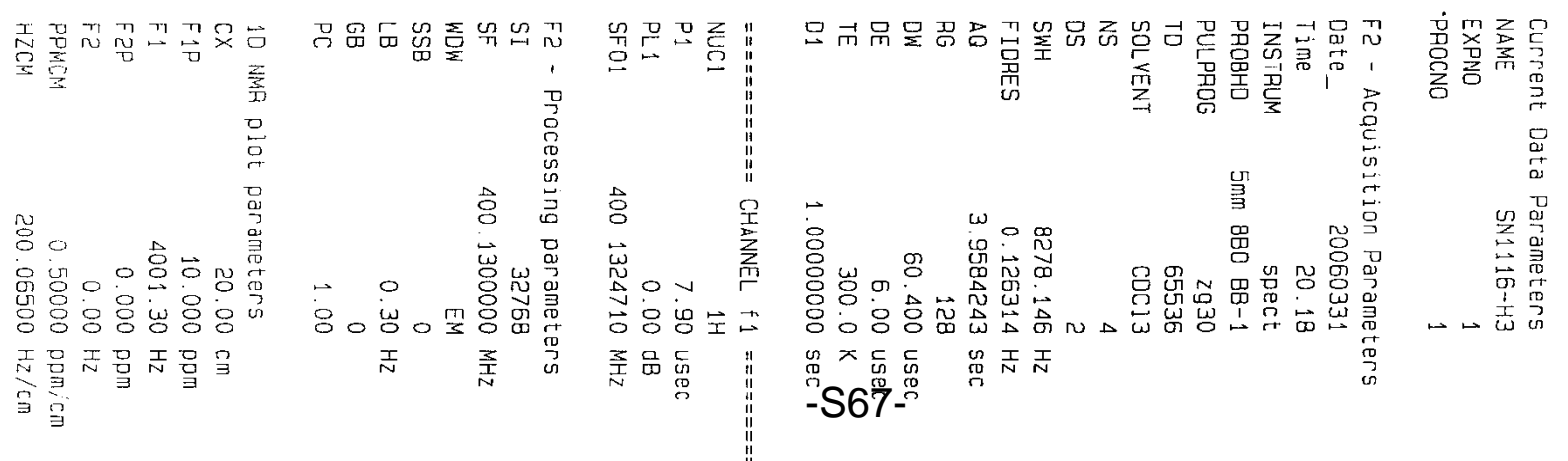




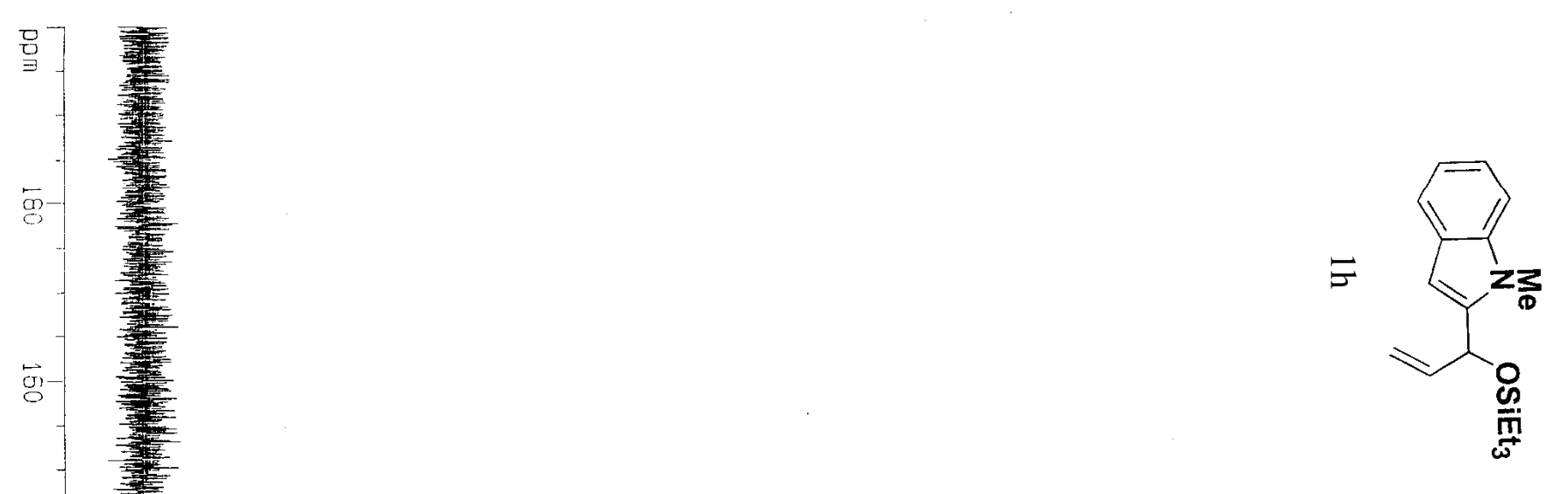

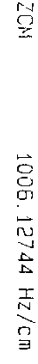

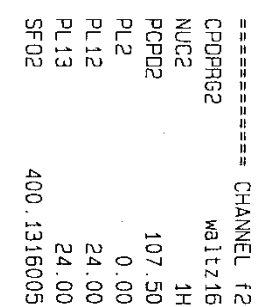

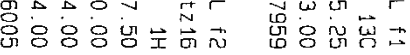
棌通品吕点

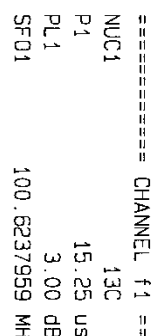

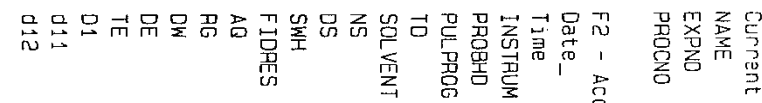
유 0 n

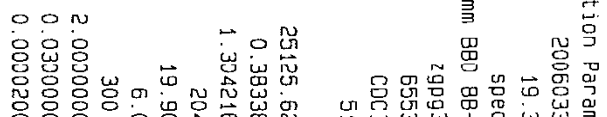

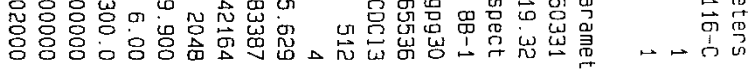

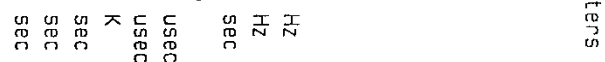

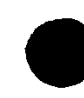



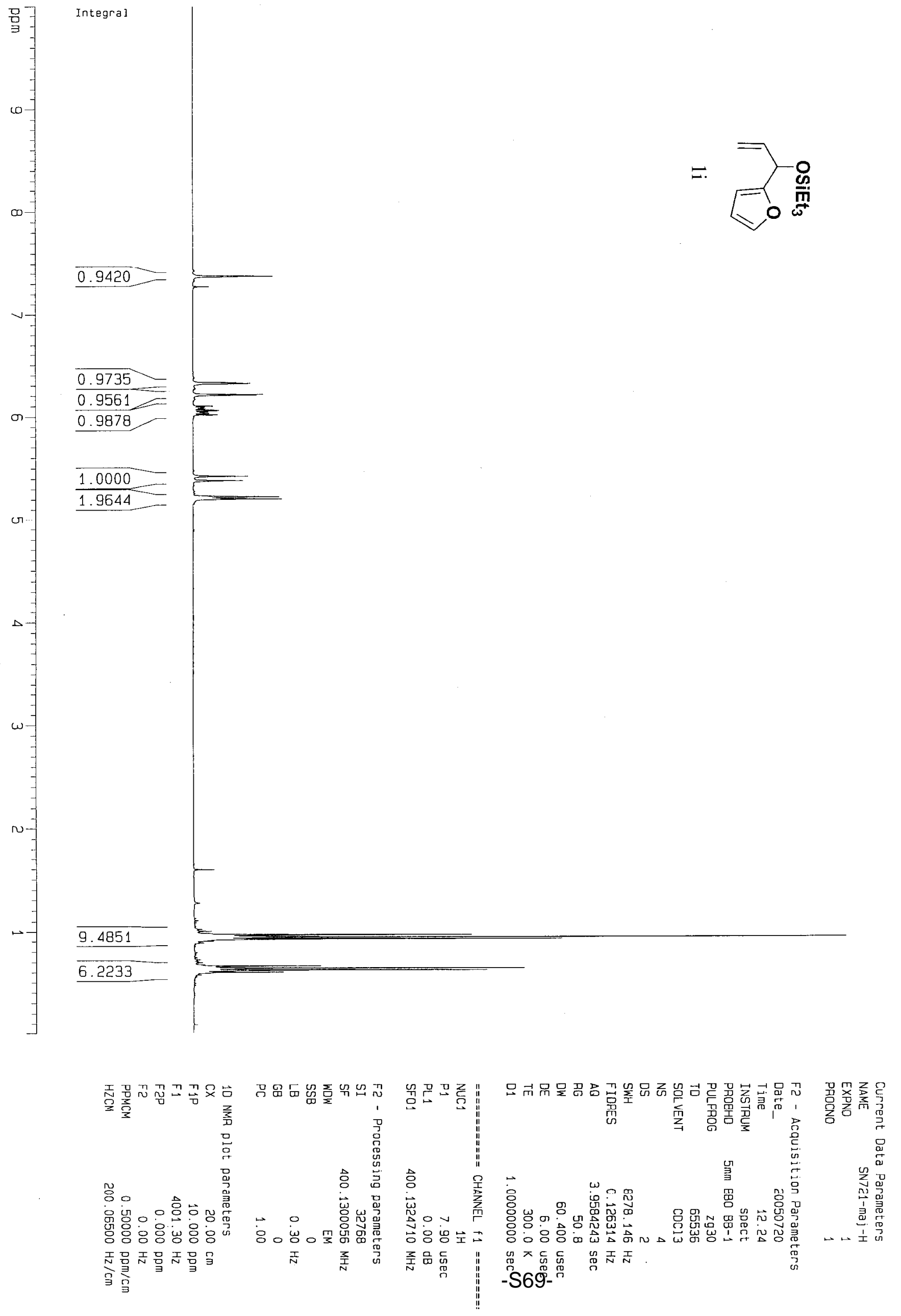

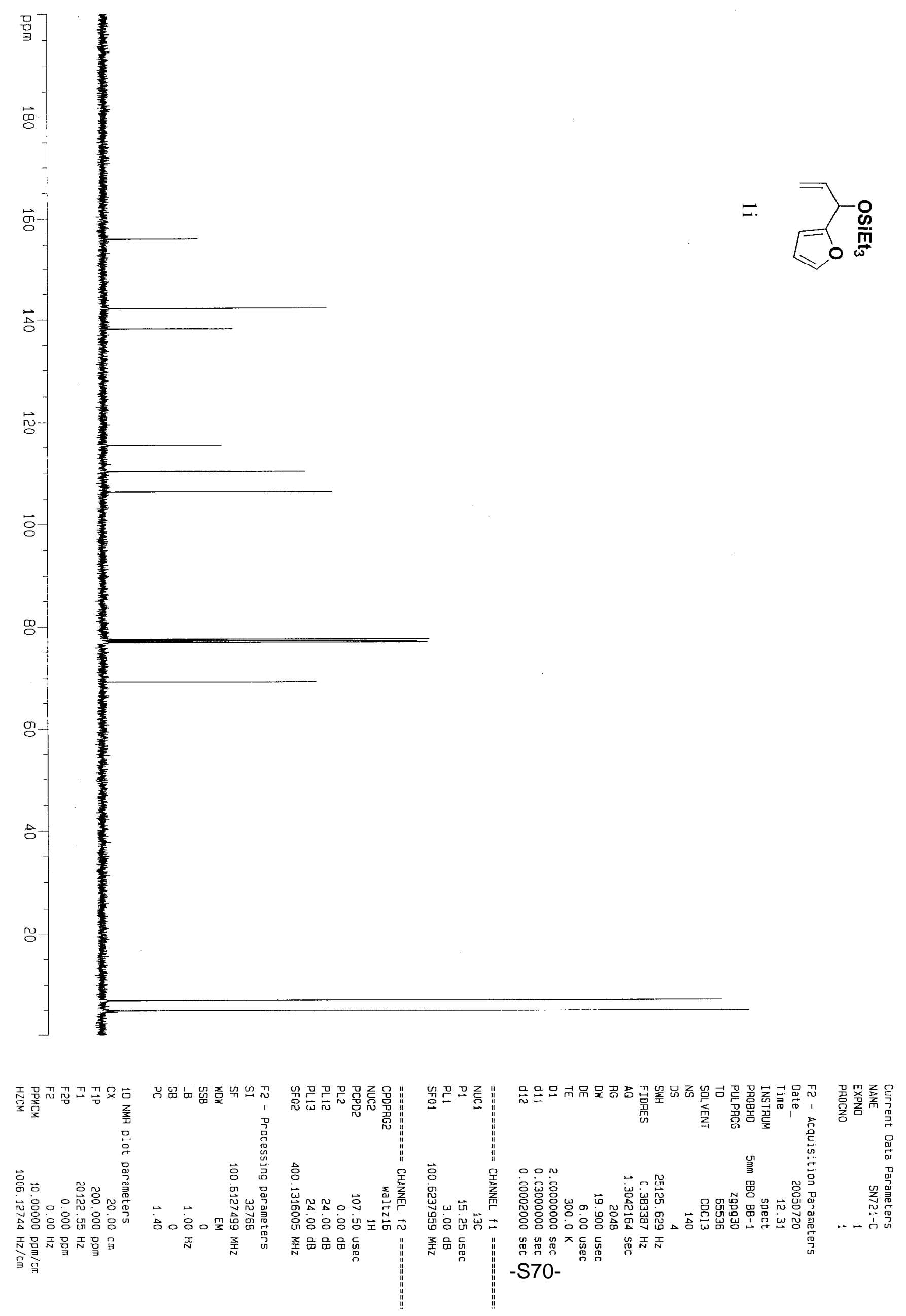

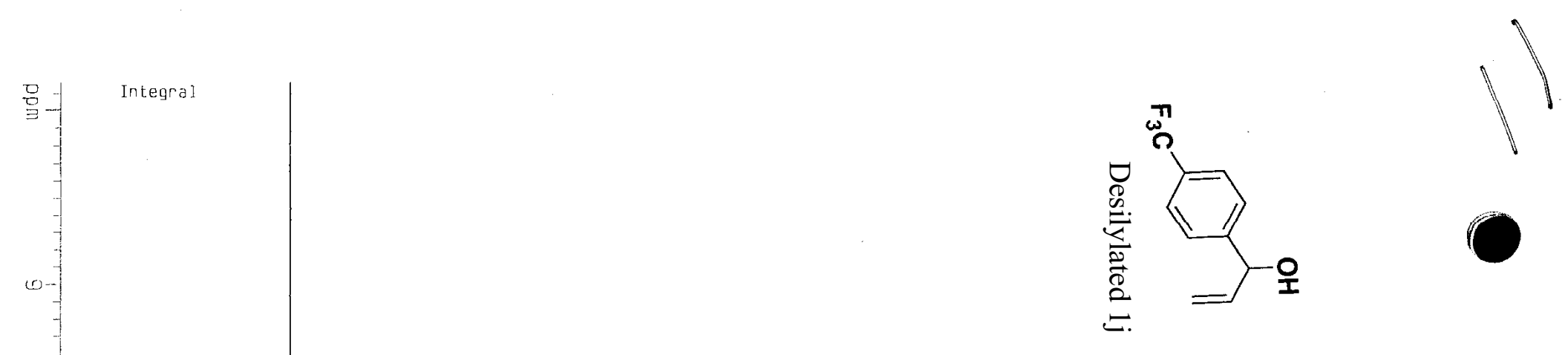

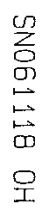
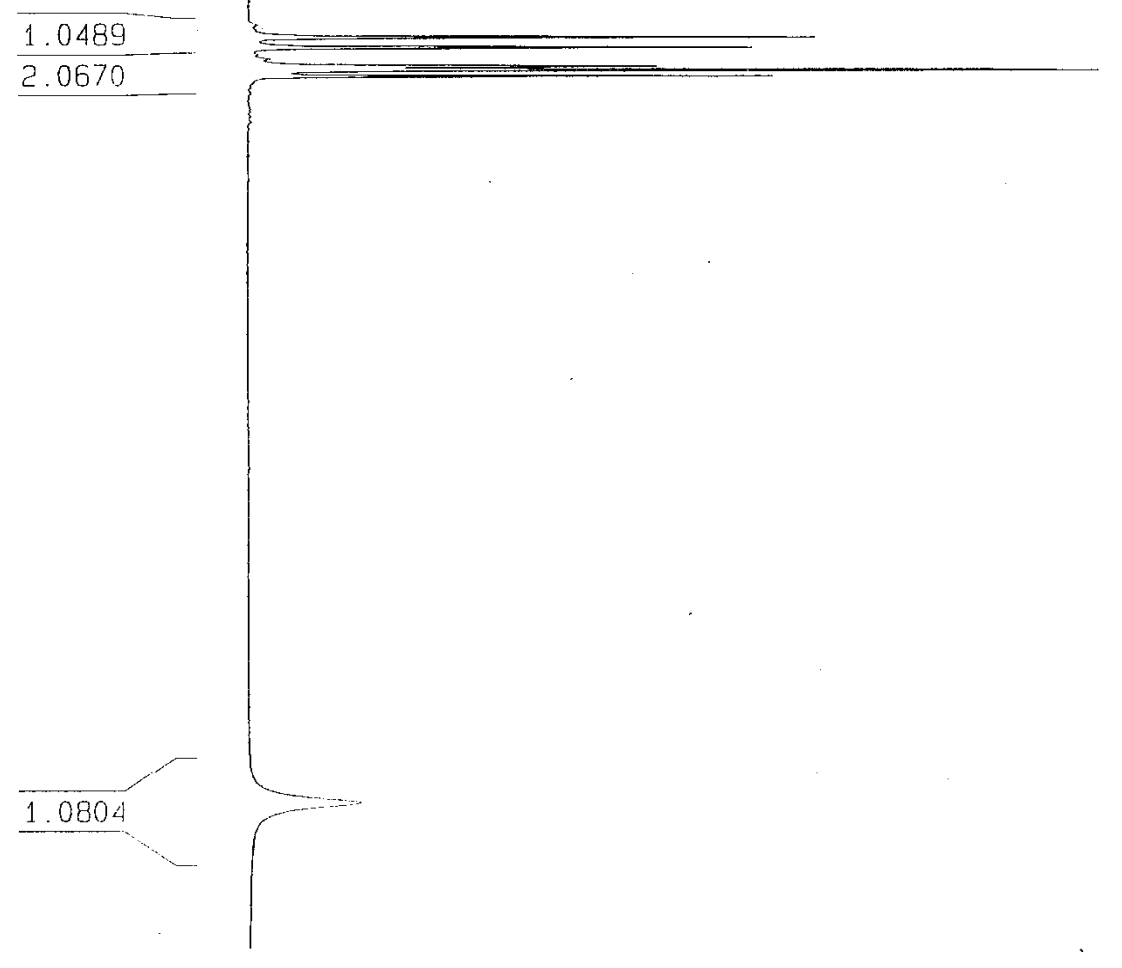

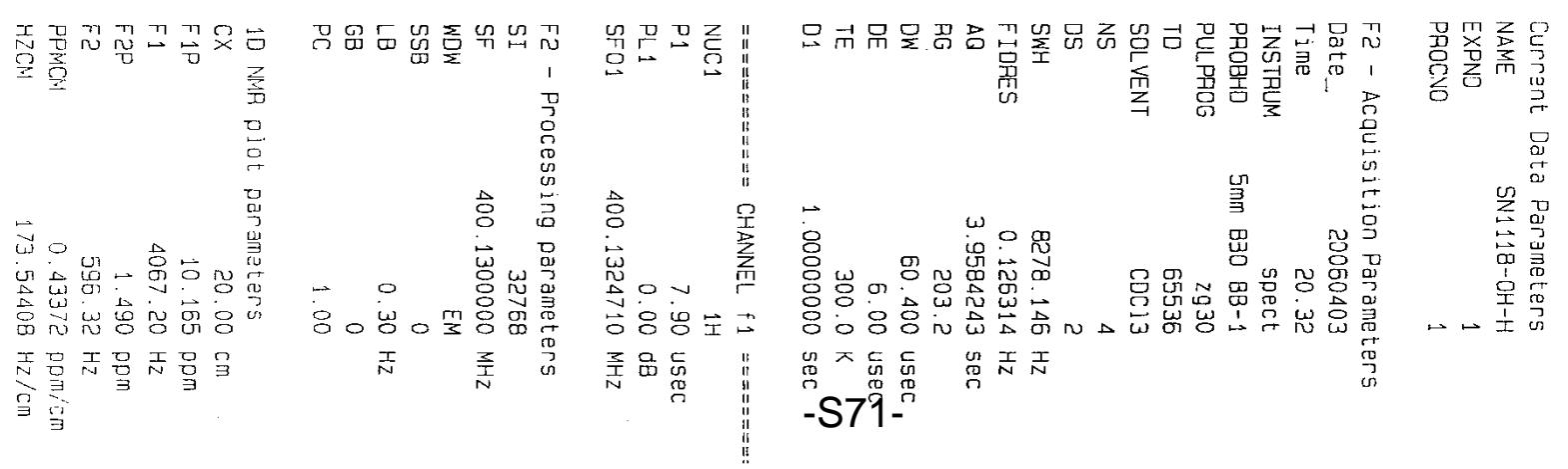



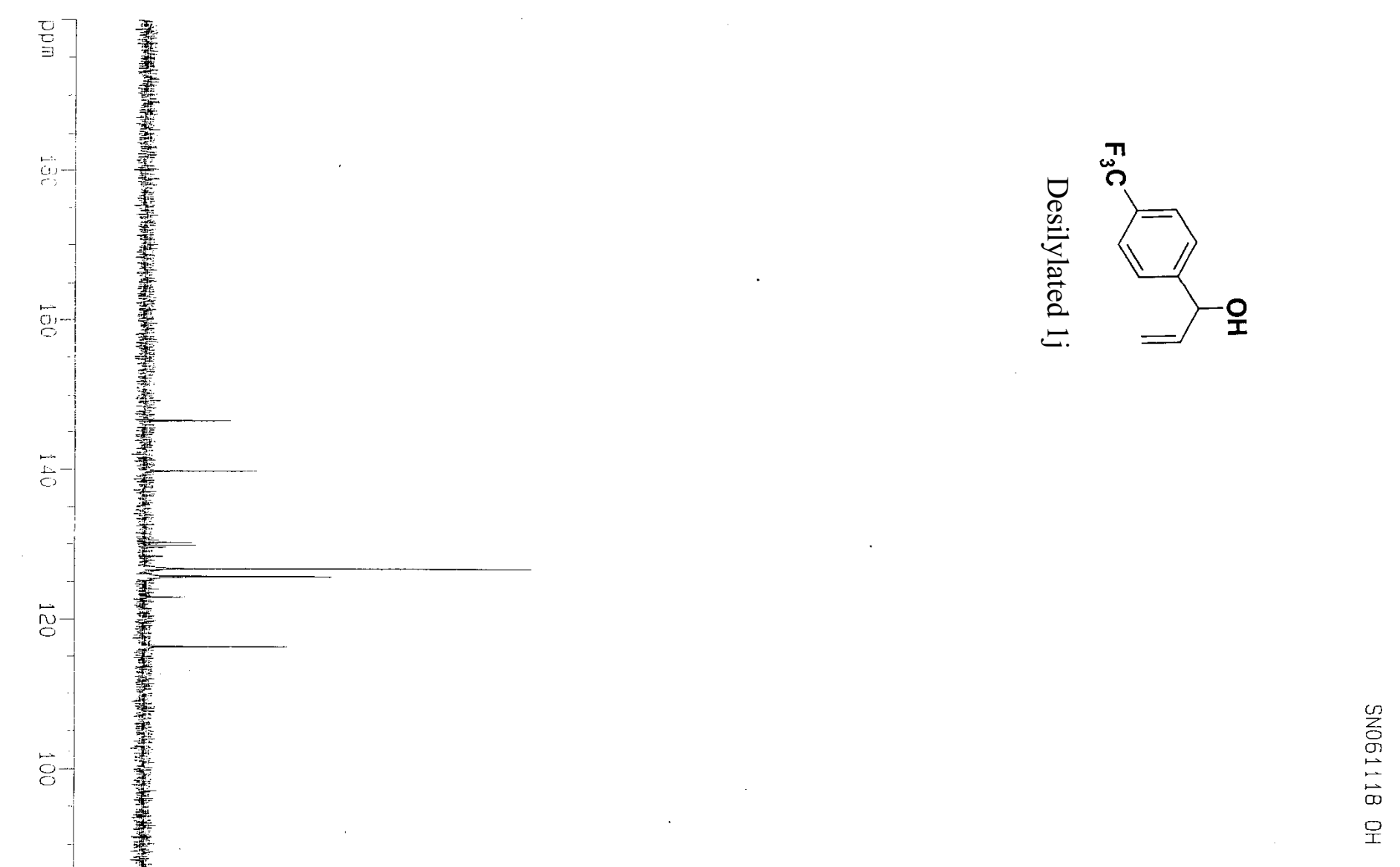

8

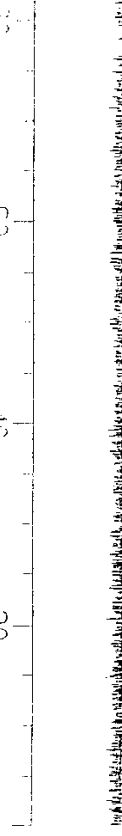



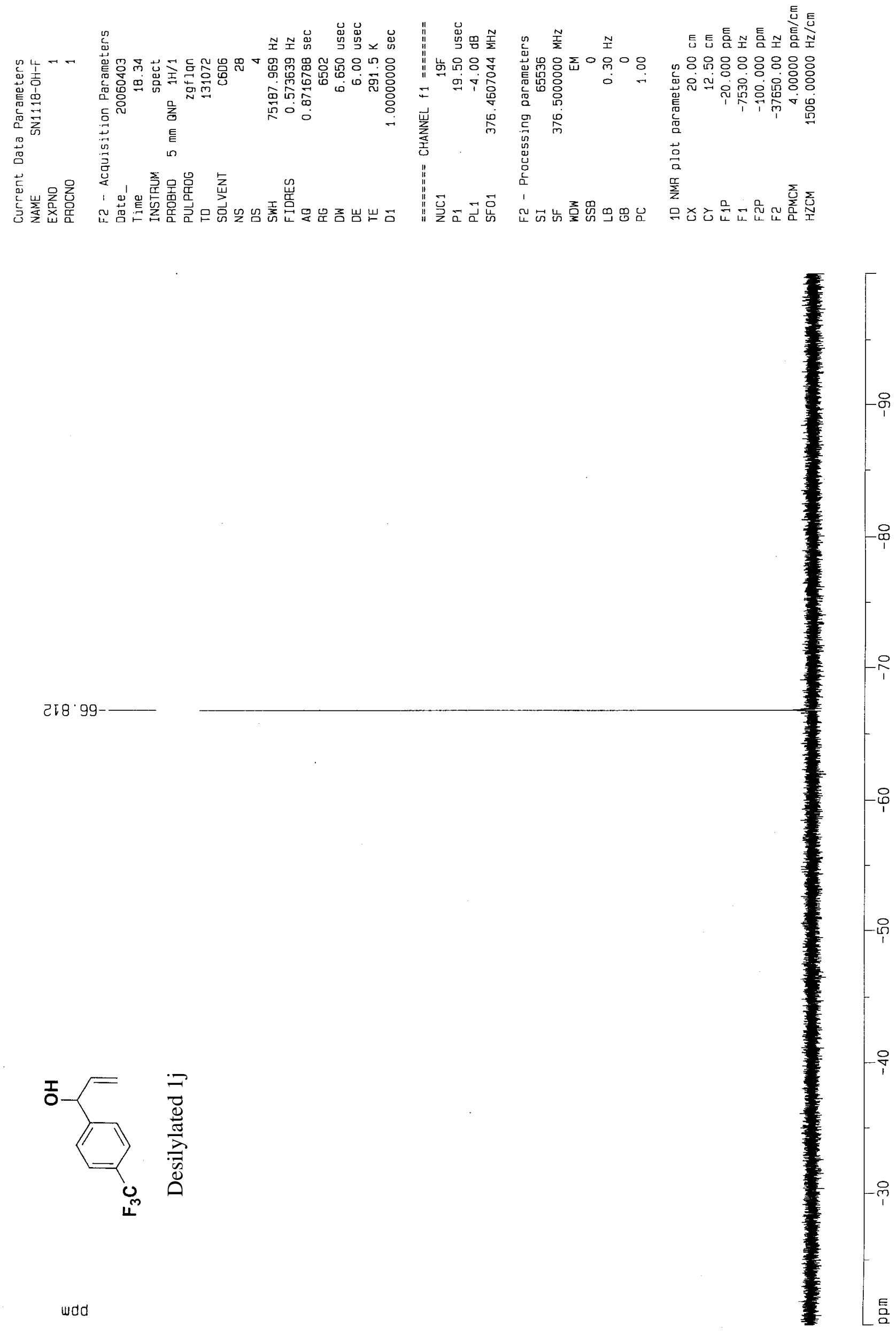

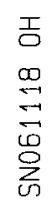



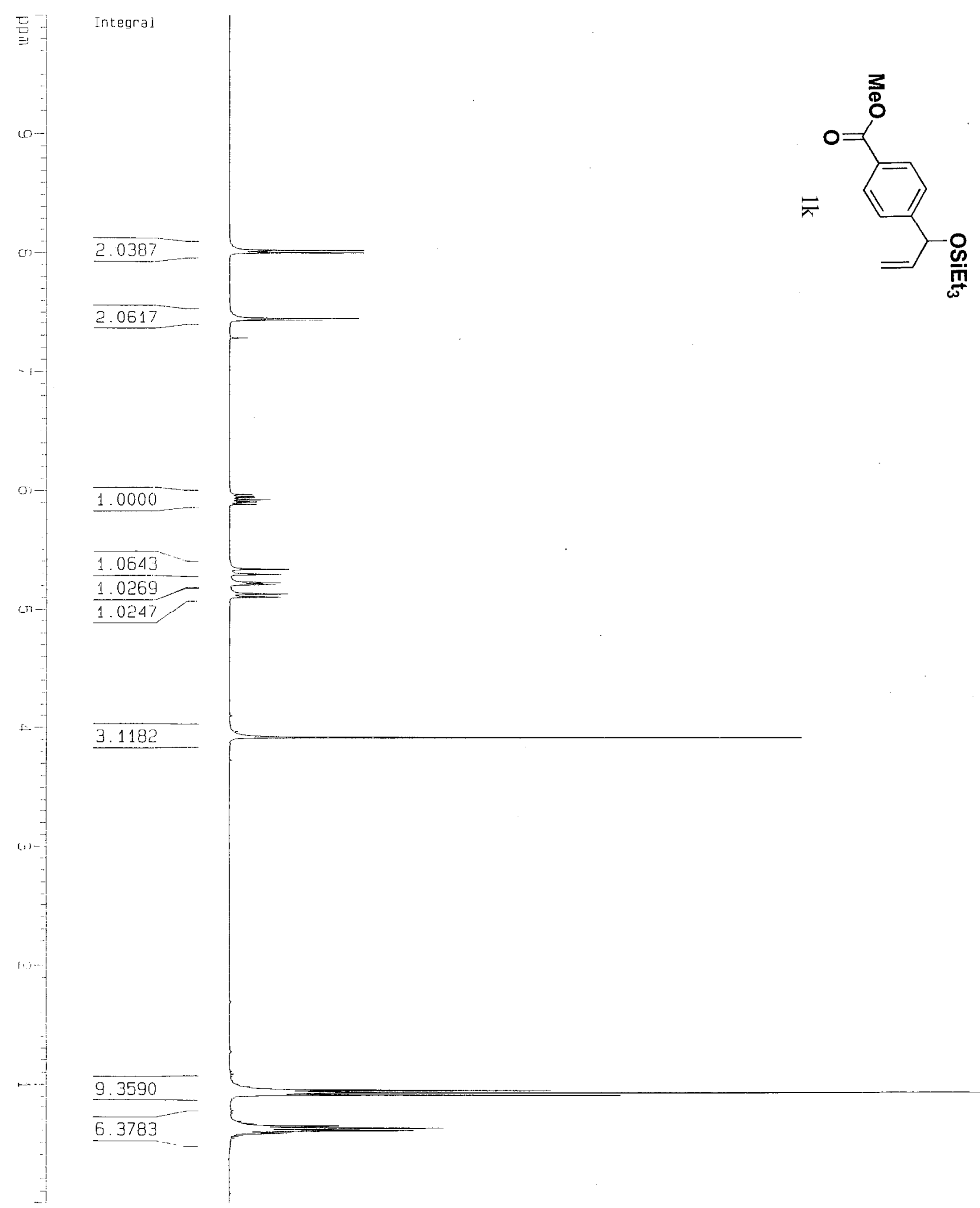

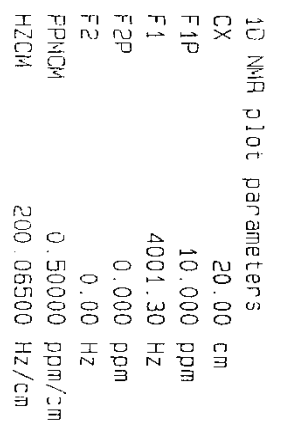
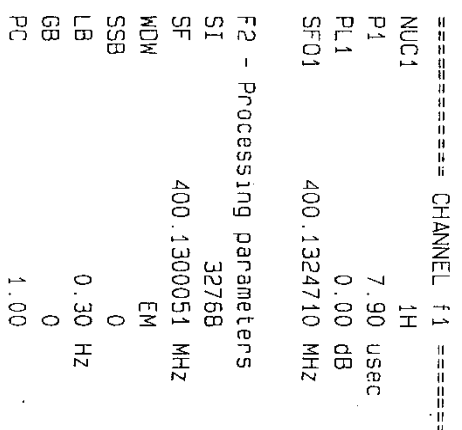

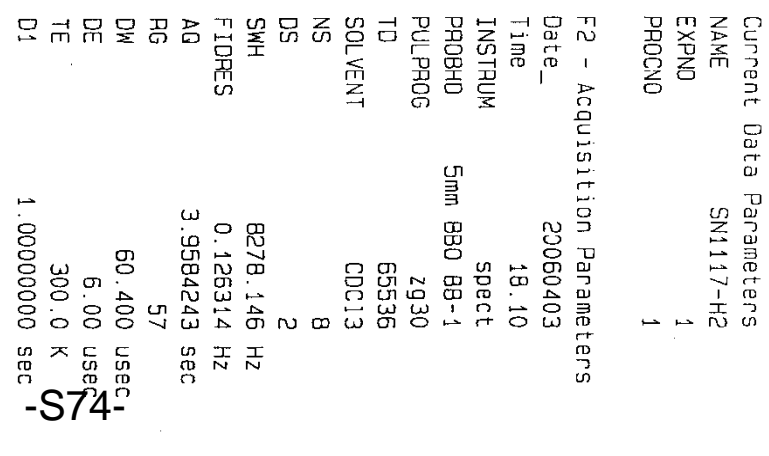




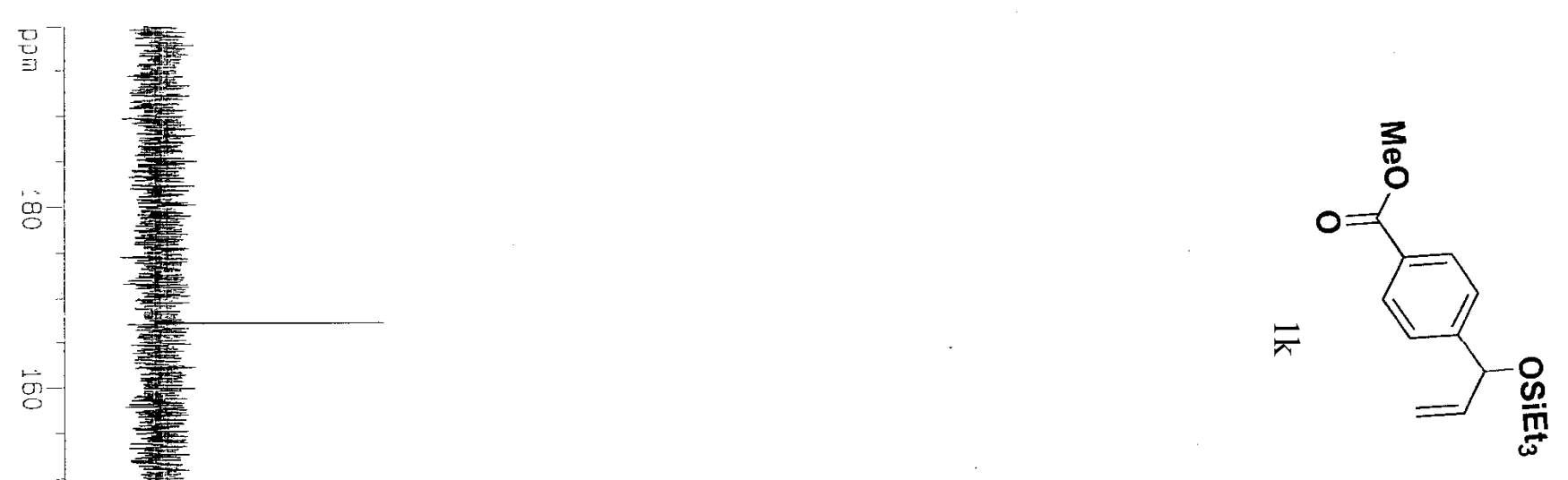




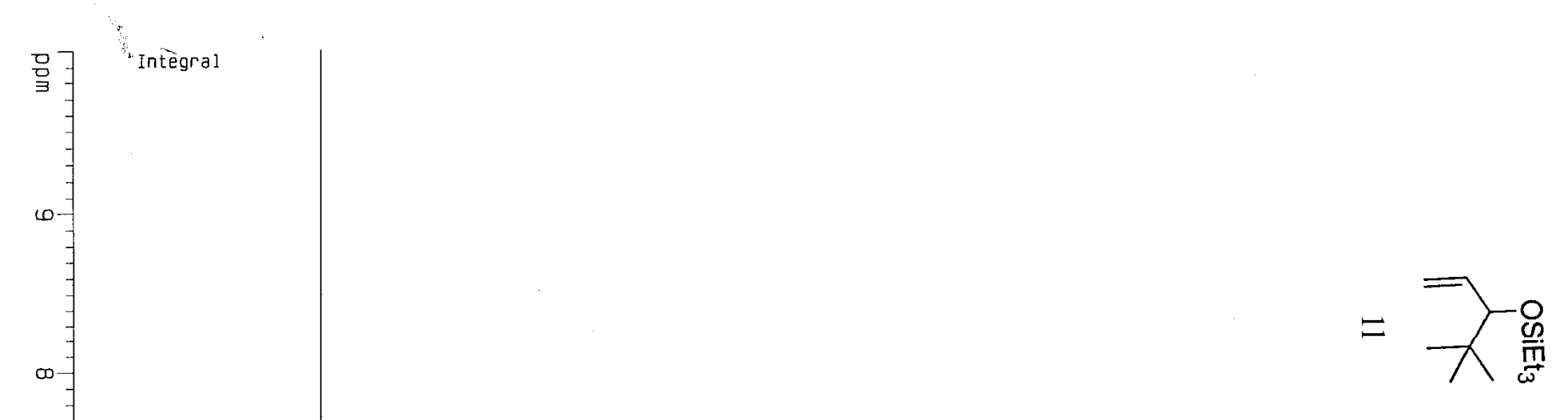

品

N

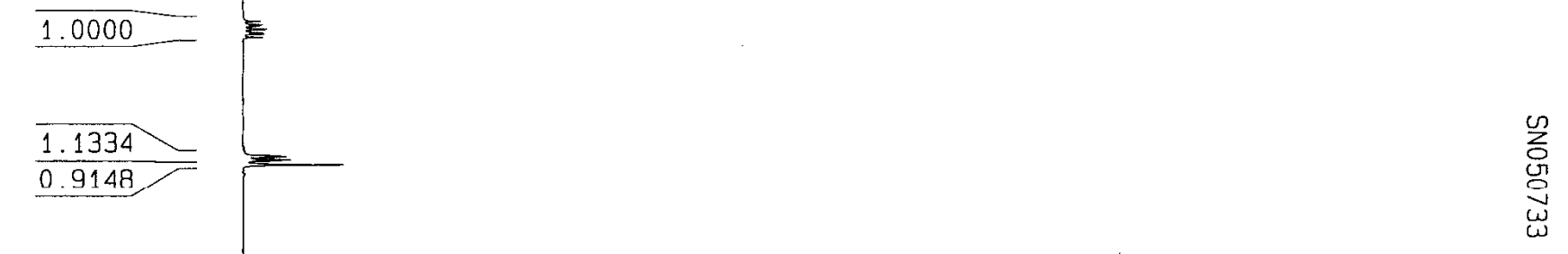

$\omega$
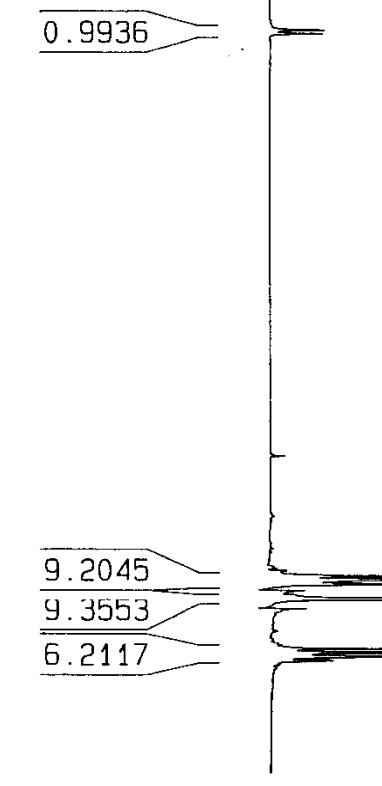

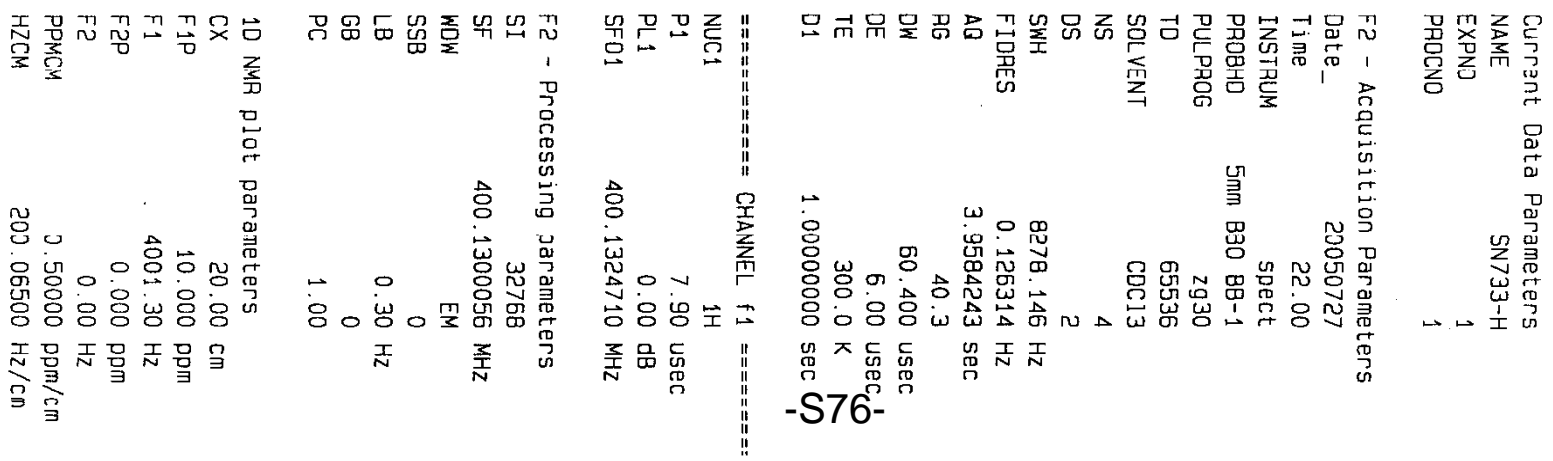




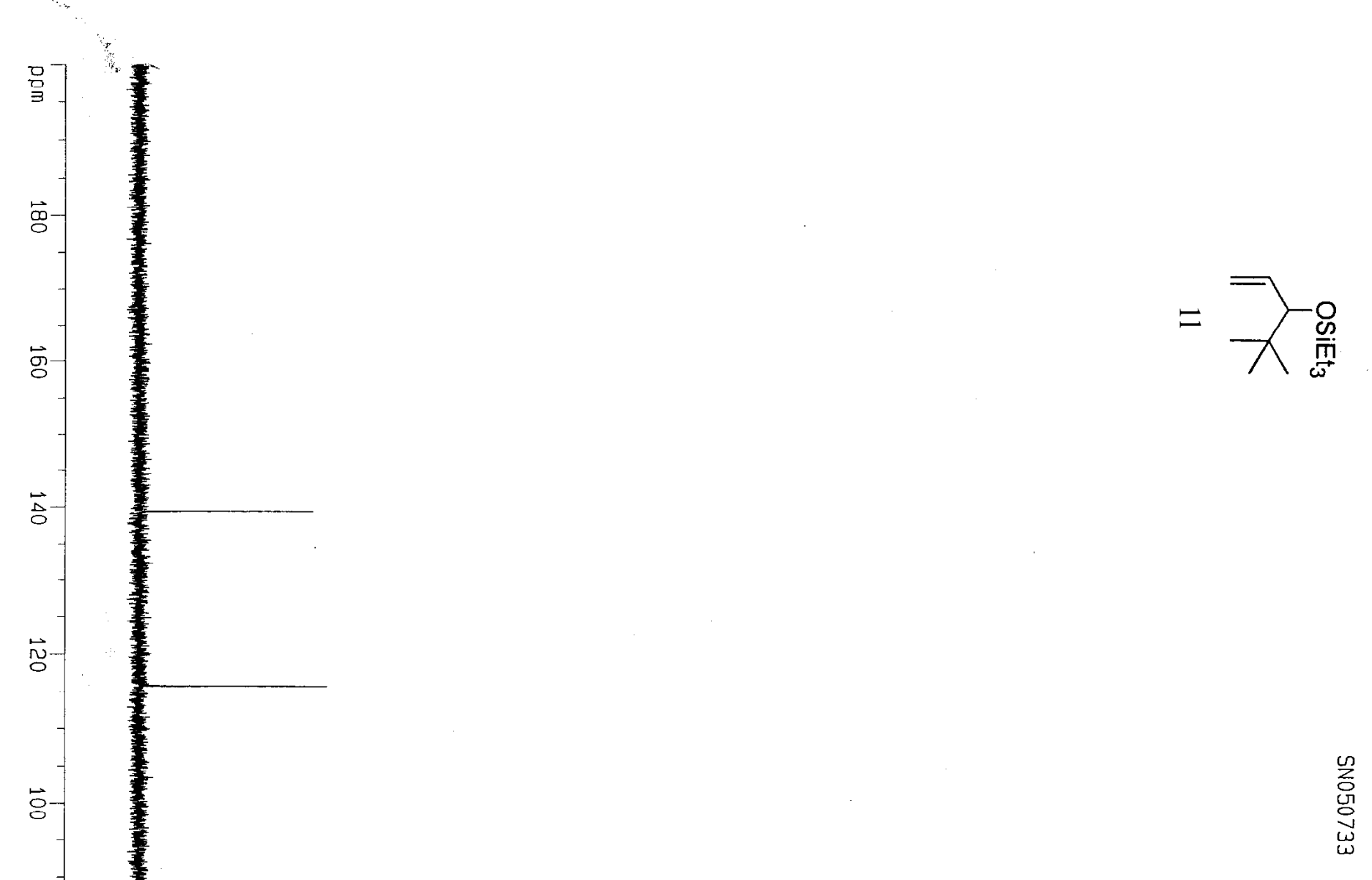

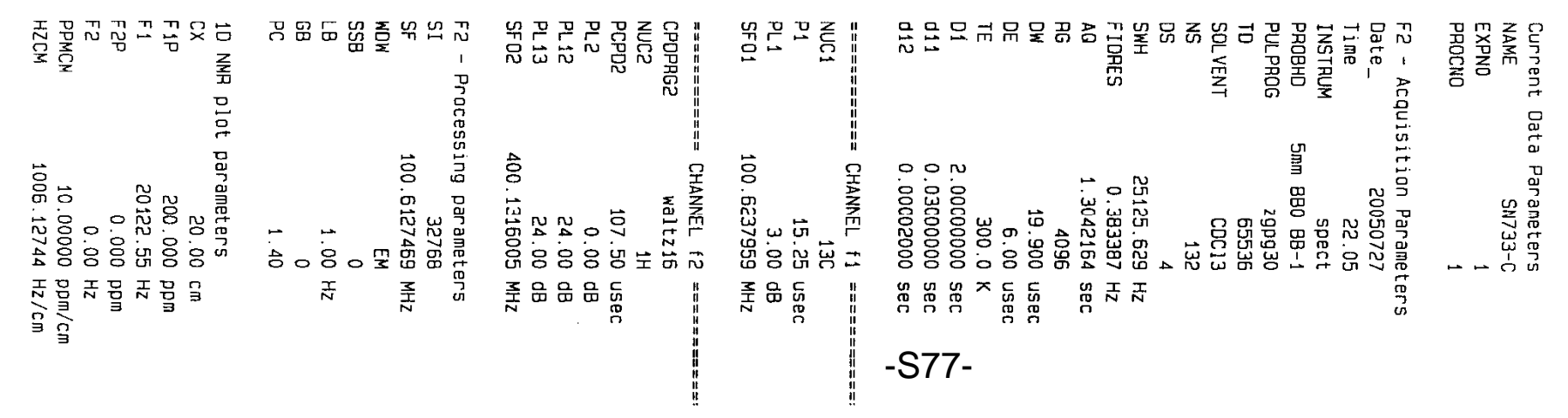

8

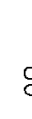

?

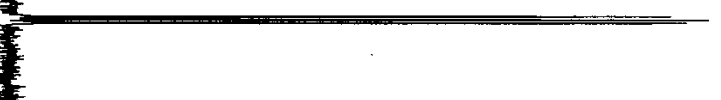

o

.

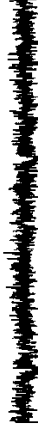



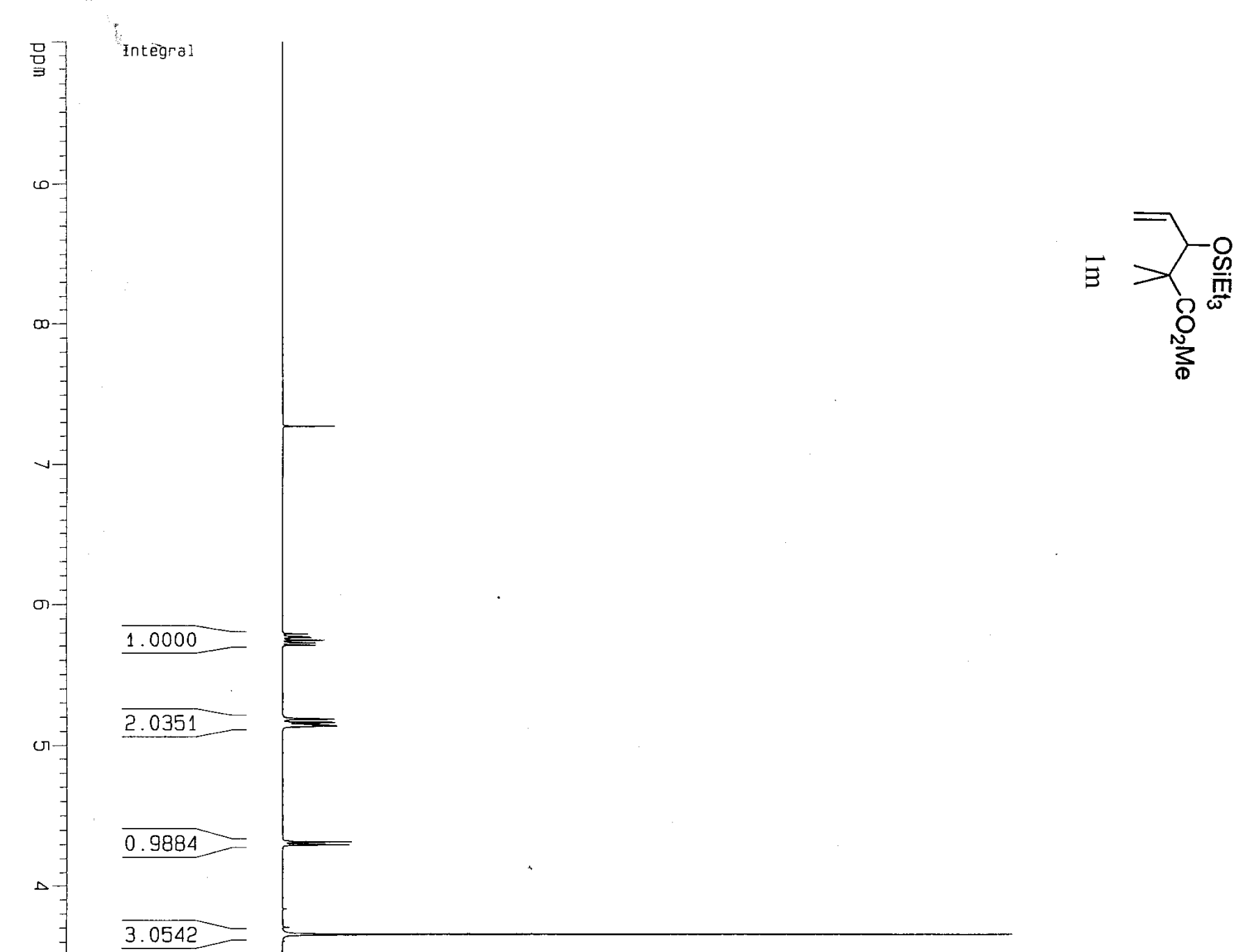

3.0893
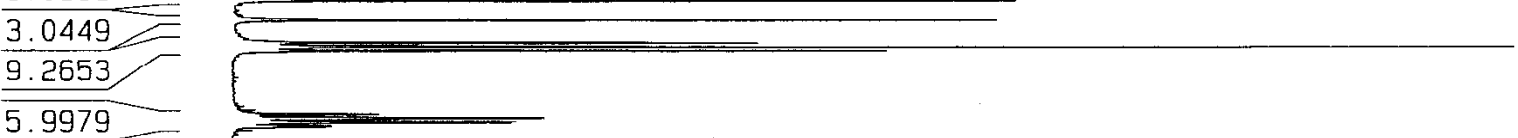

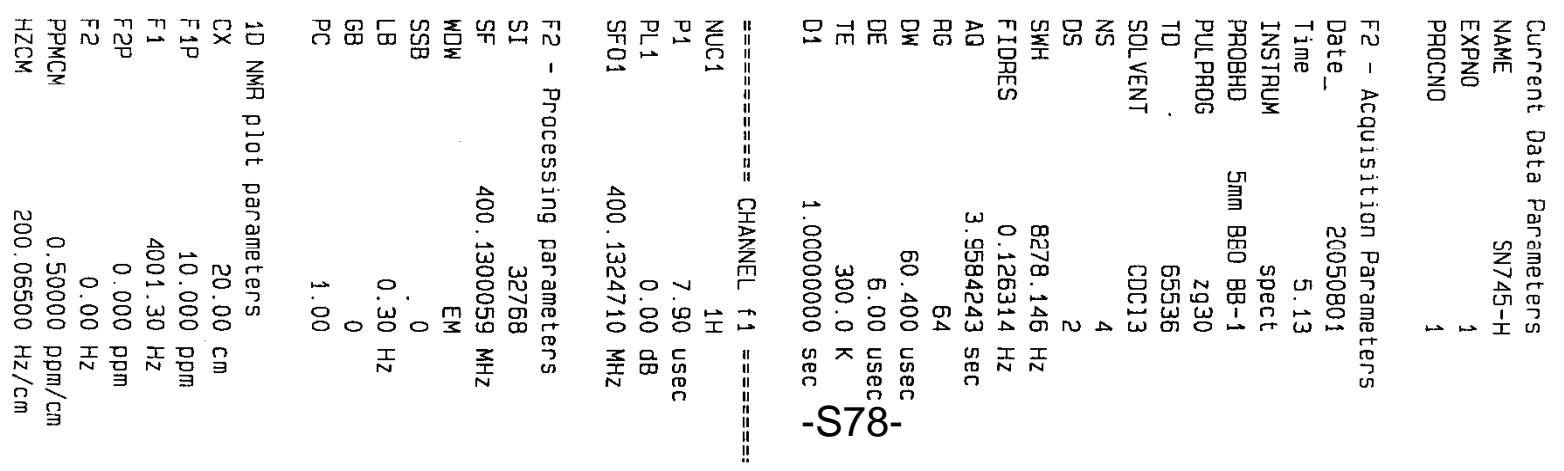





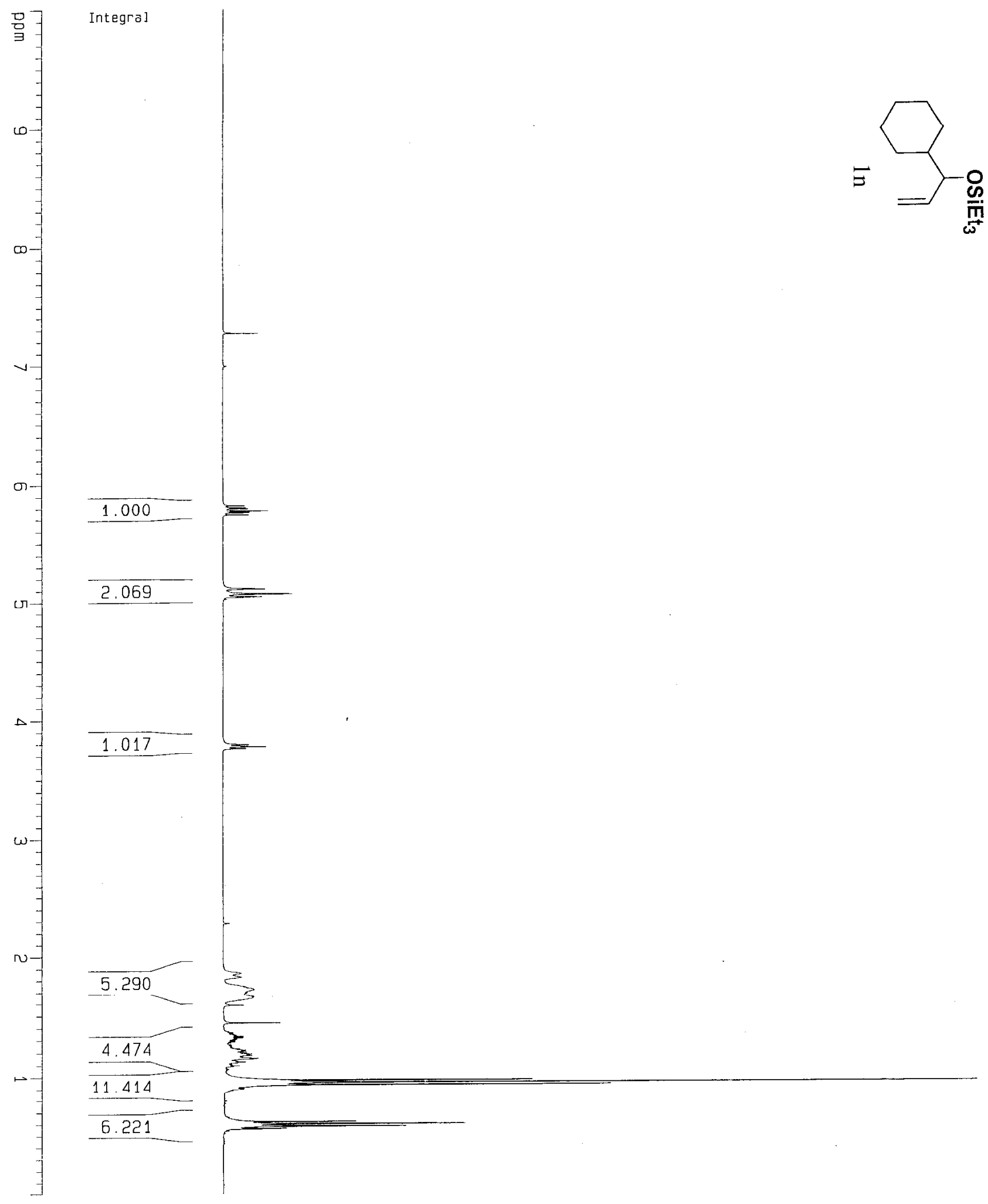


ก

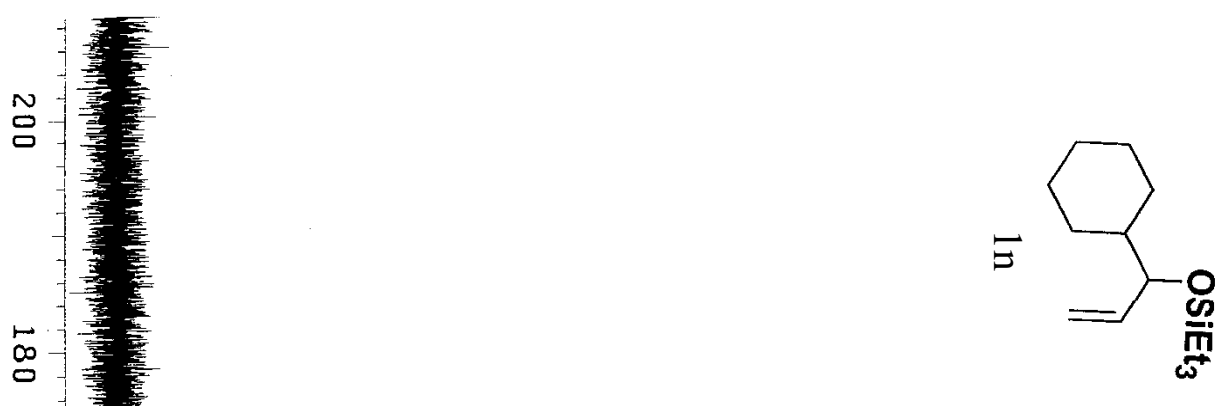

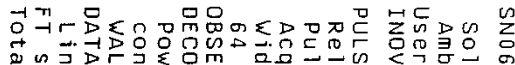

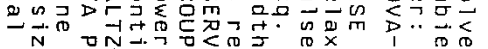

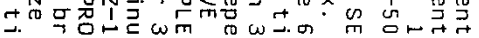

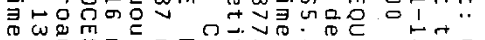

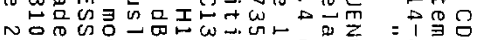

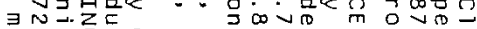

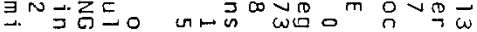

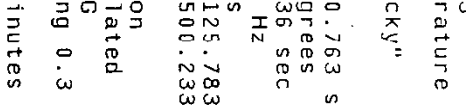

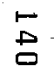

。 音

-

吕

동

-

$\stackrel{\infty}{\circ}$

(t)

g

: 

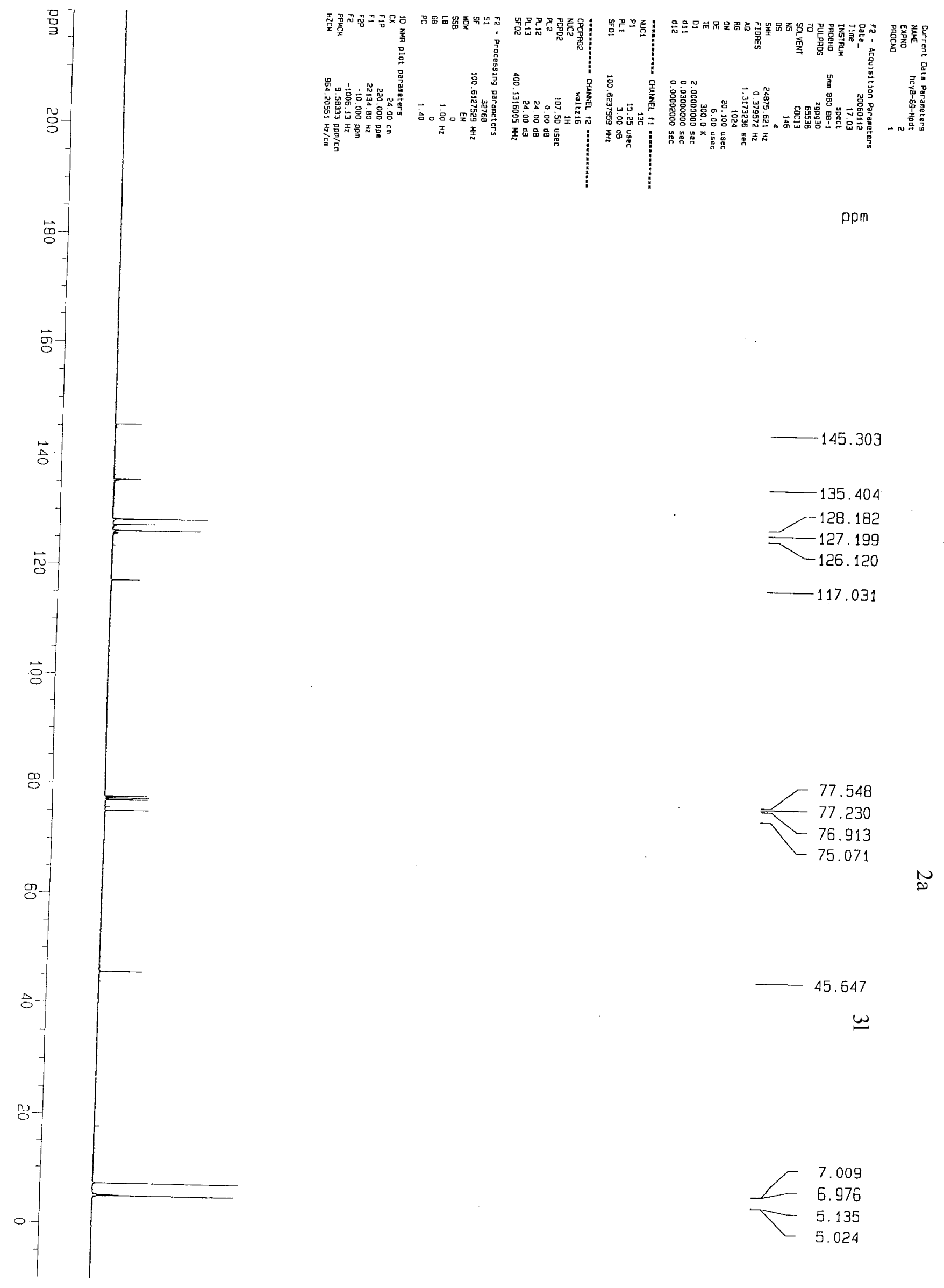

ppm

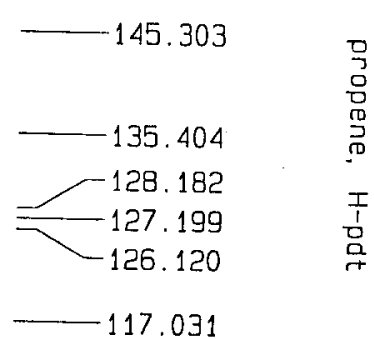

章
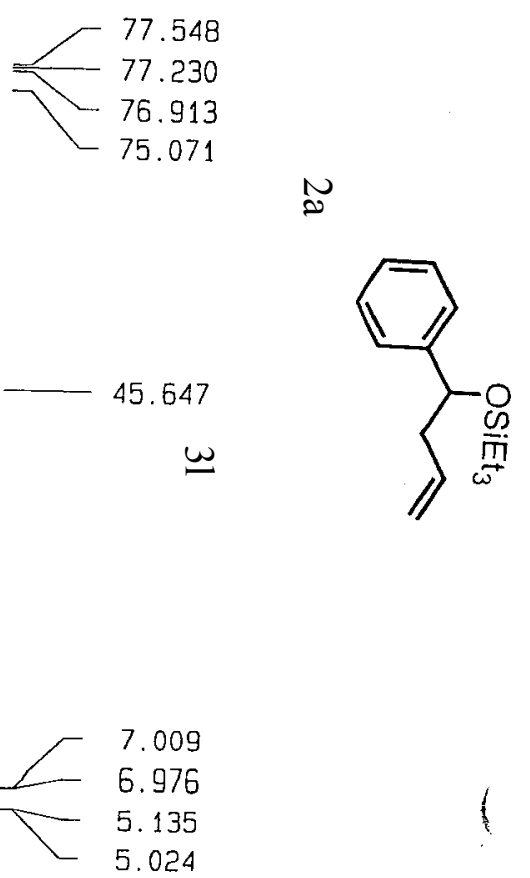


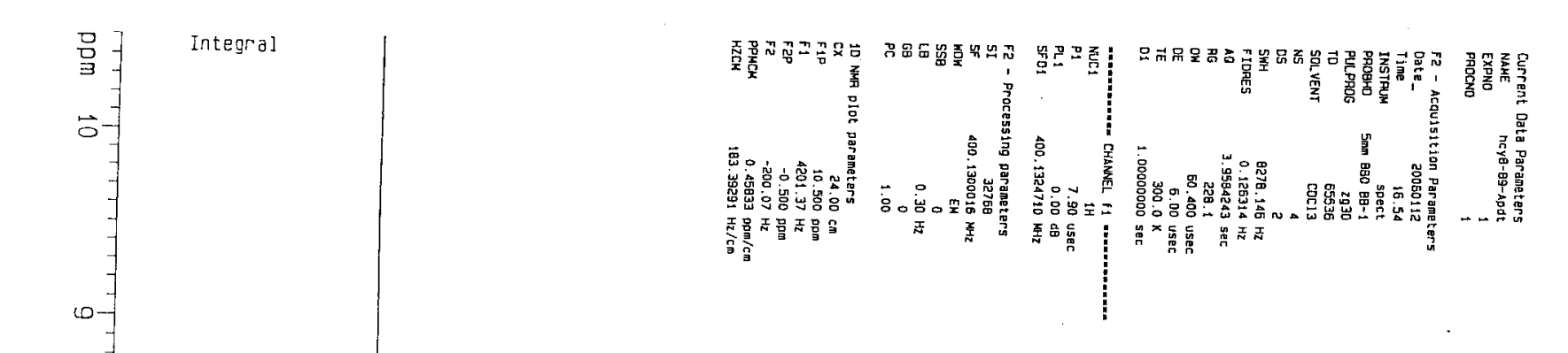

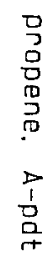
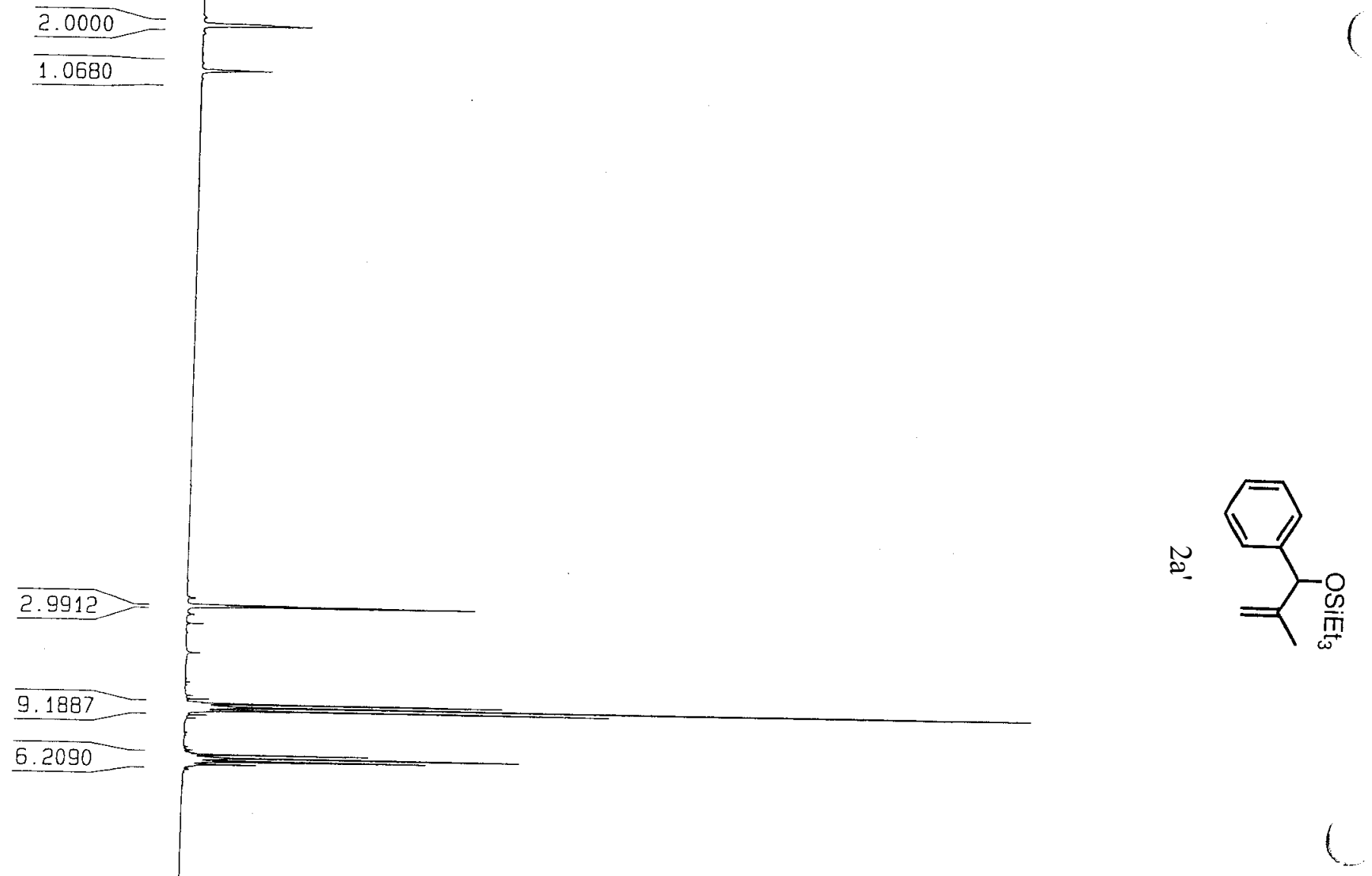


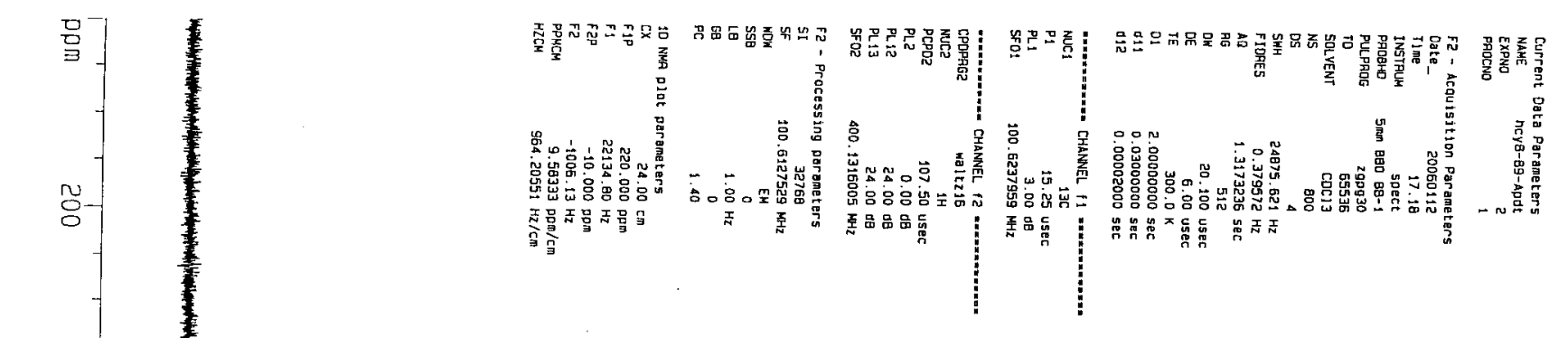

ppm
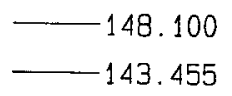

$\overleftrightarrow{\Delta}$

항-

茴-
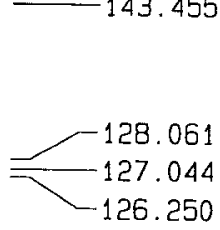

$-110.950$

客

\section{뭉}

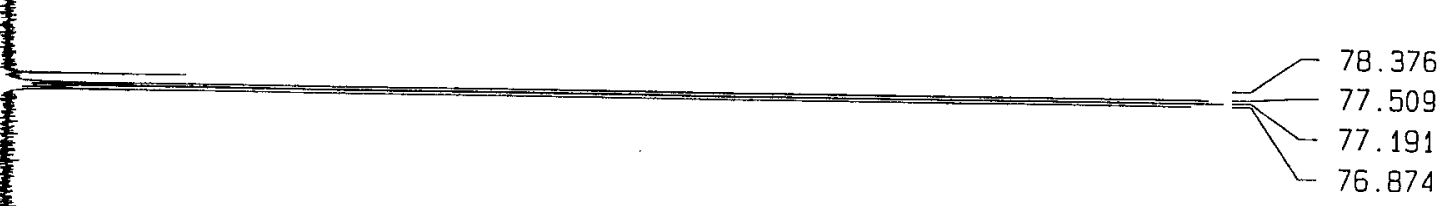

8
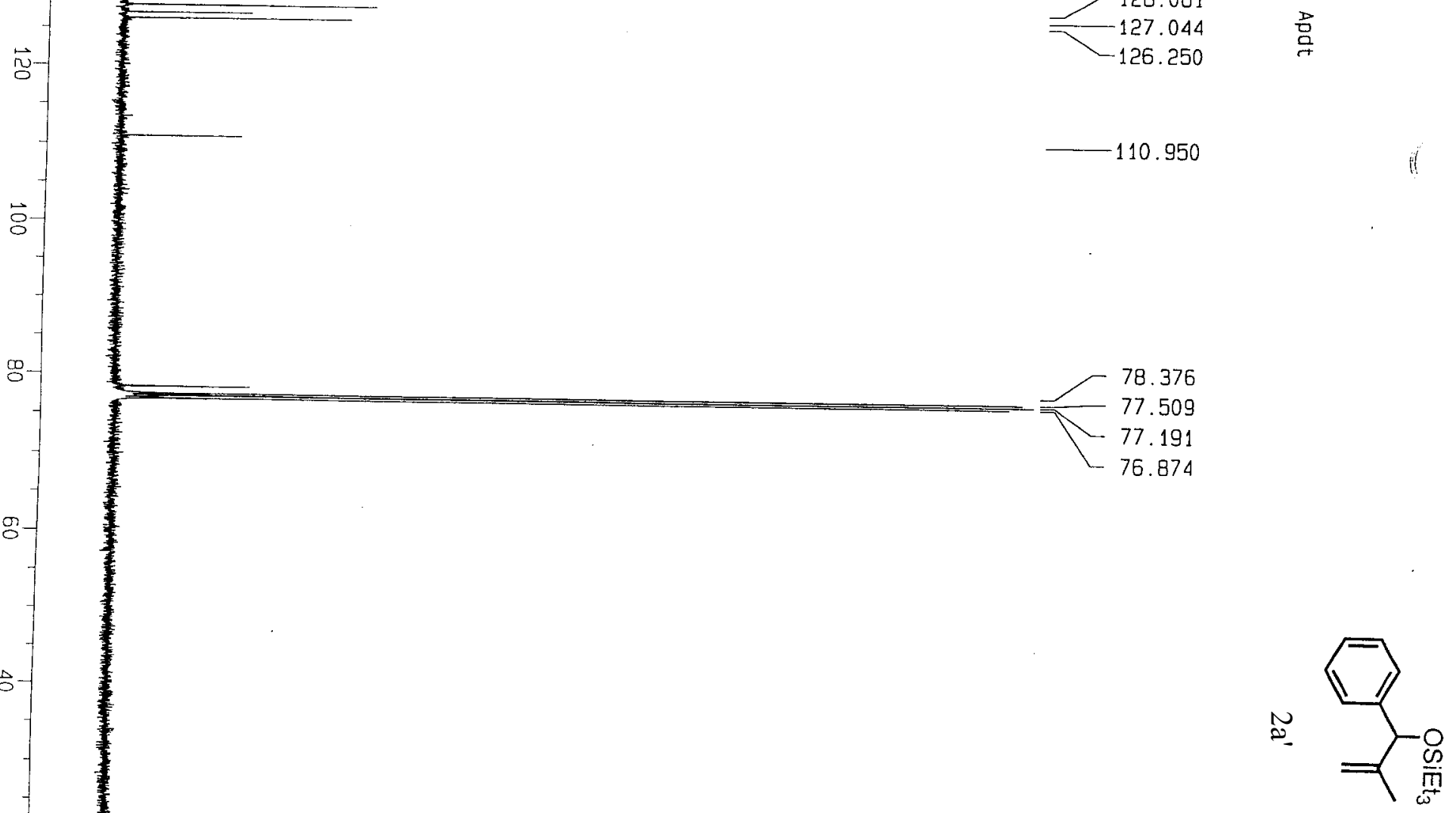

17. 435

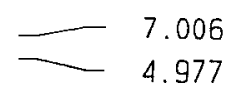




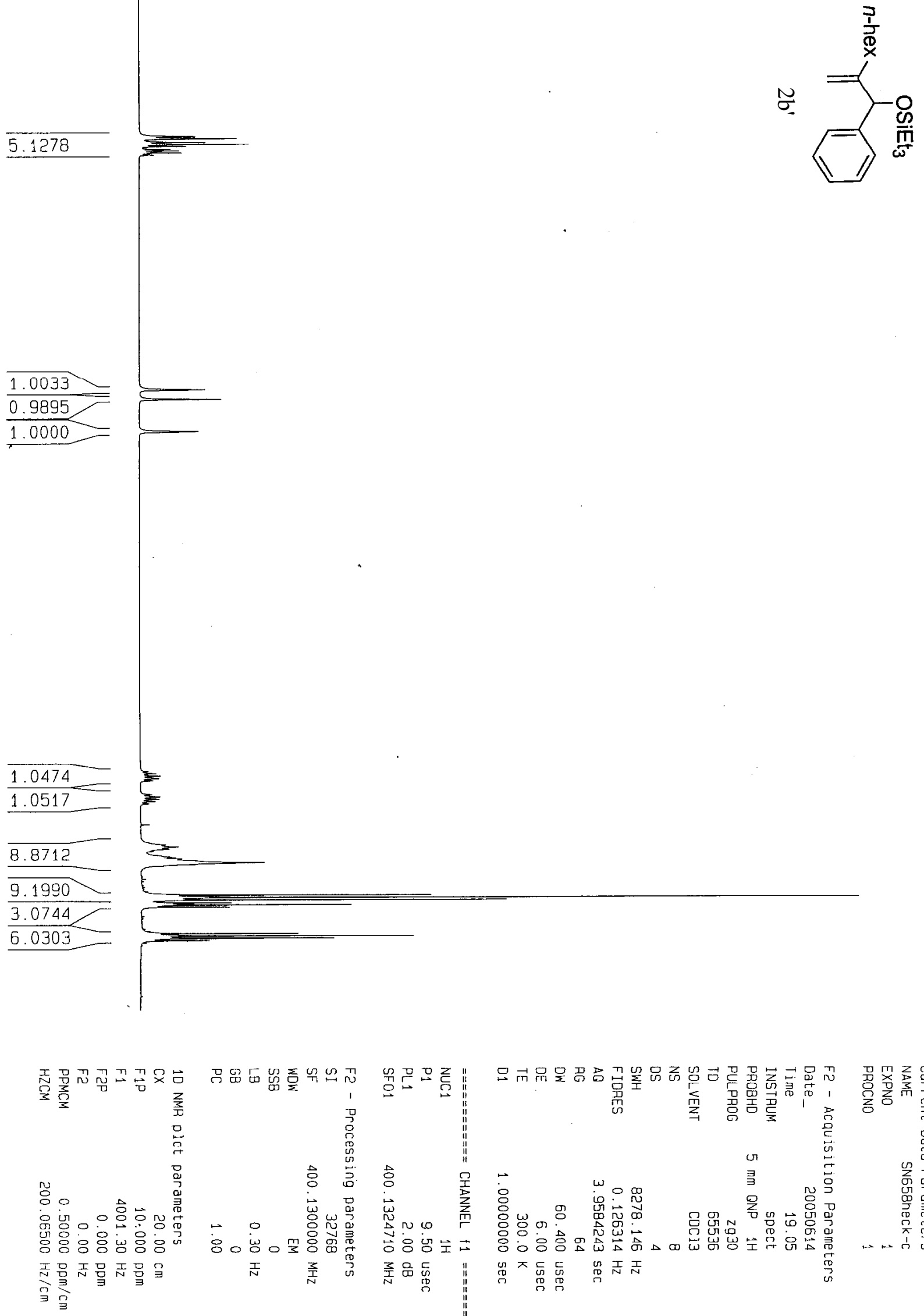

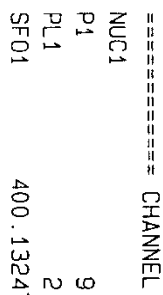

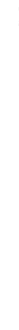

范客语空二

고N 뭄데

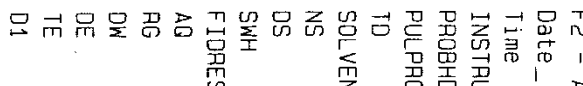

号署美号

$\dot{0} \quad$ wo $\quad$ 品

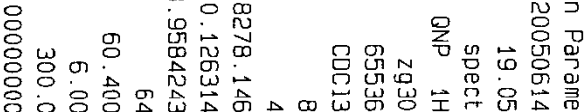

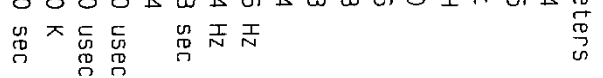

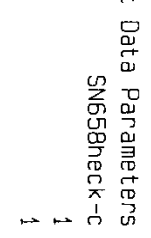

-S88- 


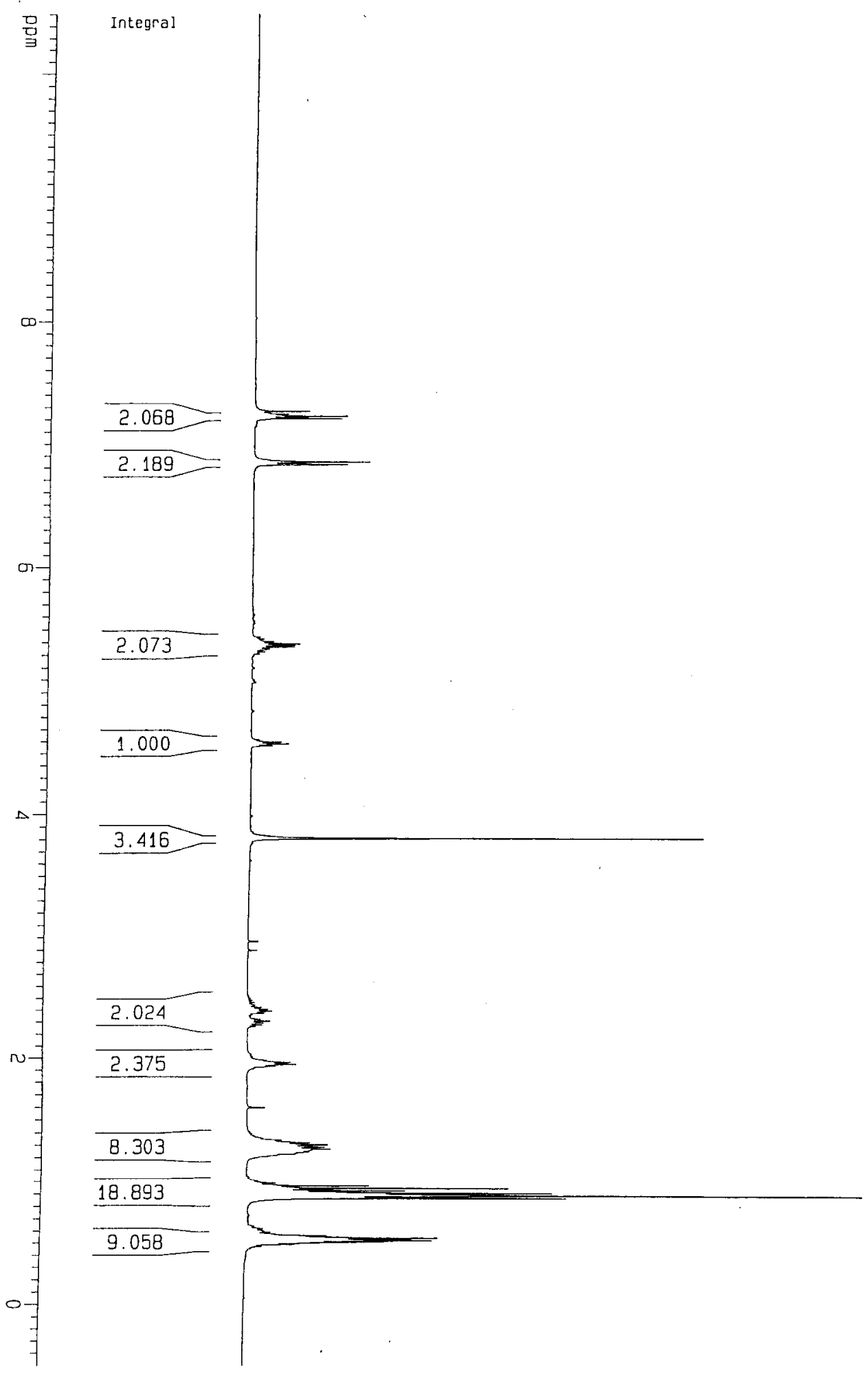

욿

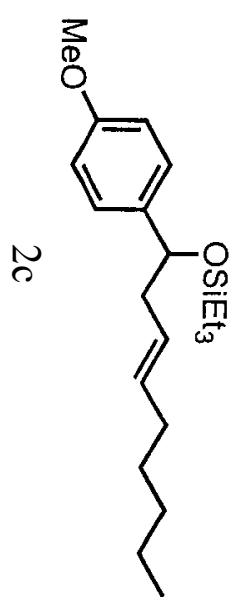

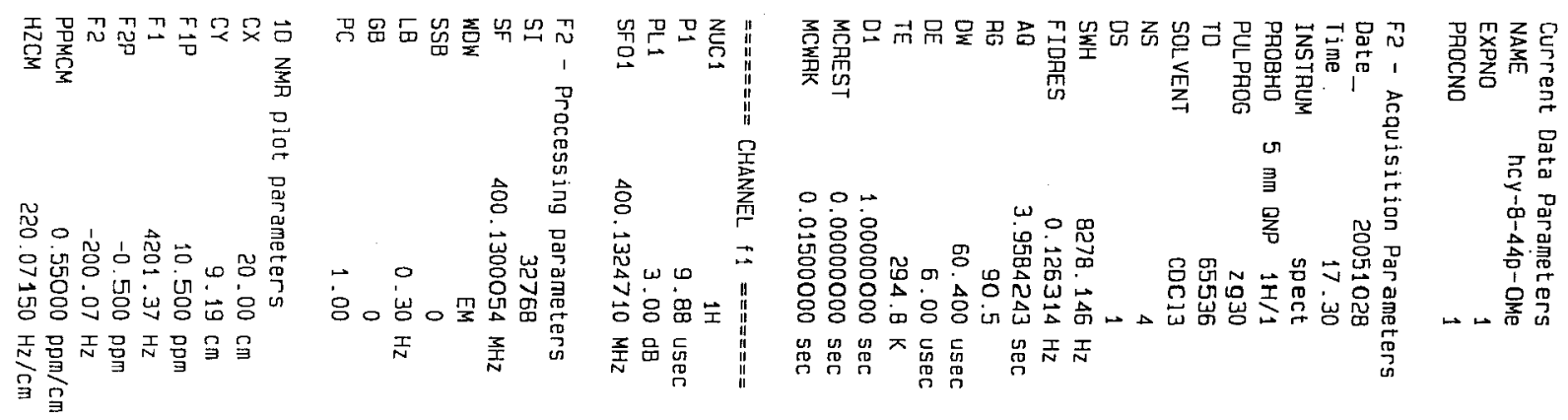




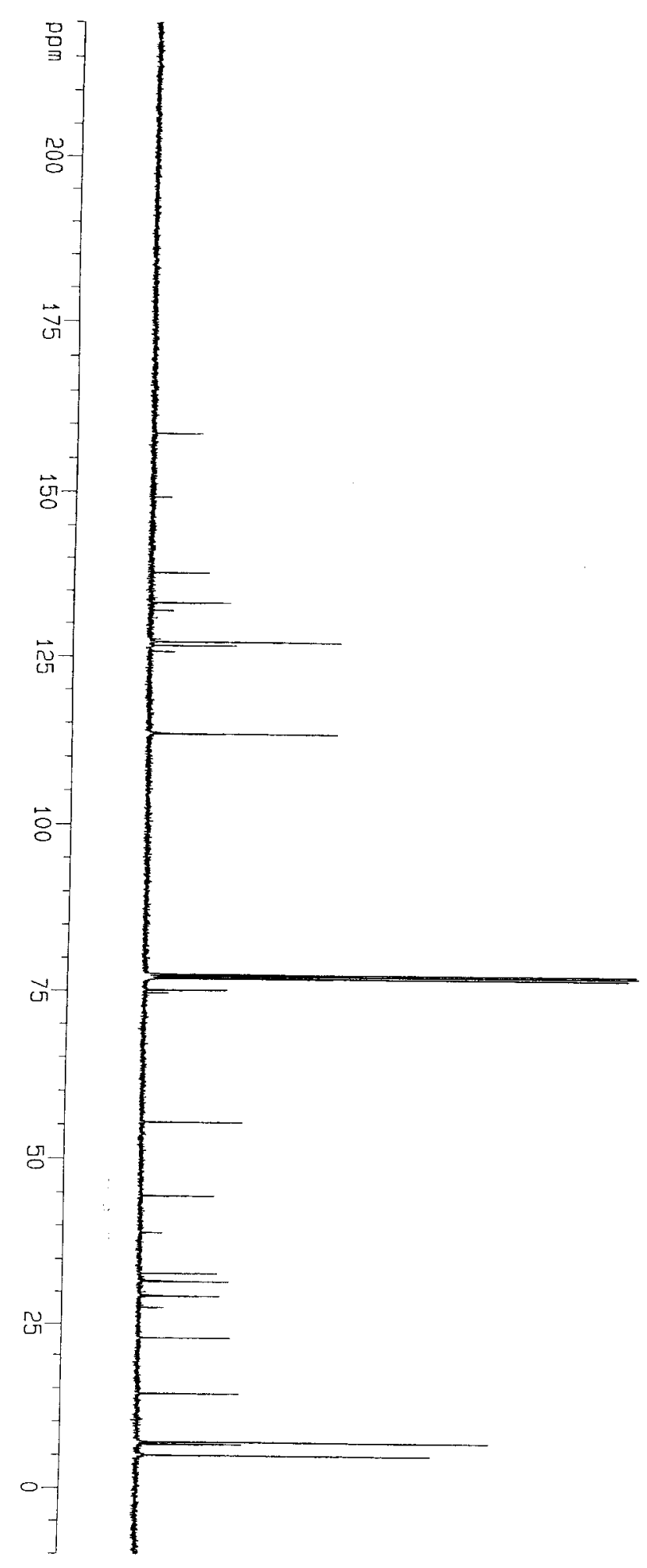

158.665

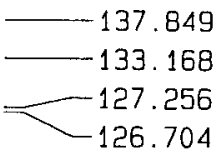

$-113.417$

욲
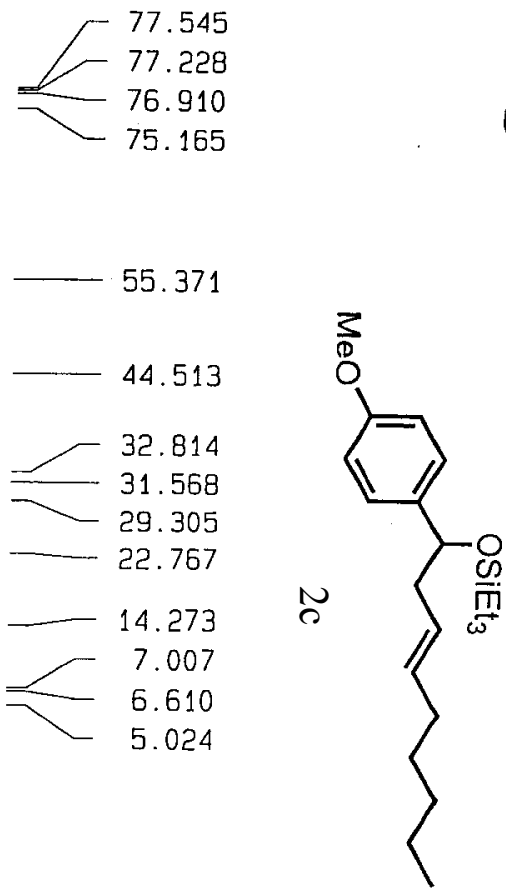

곶
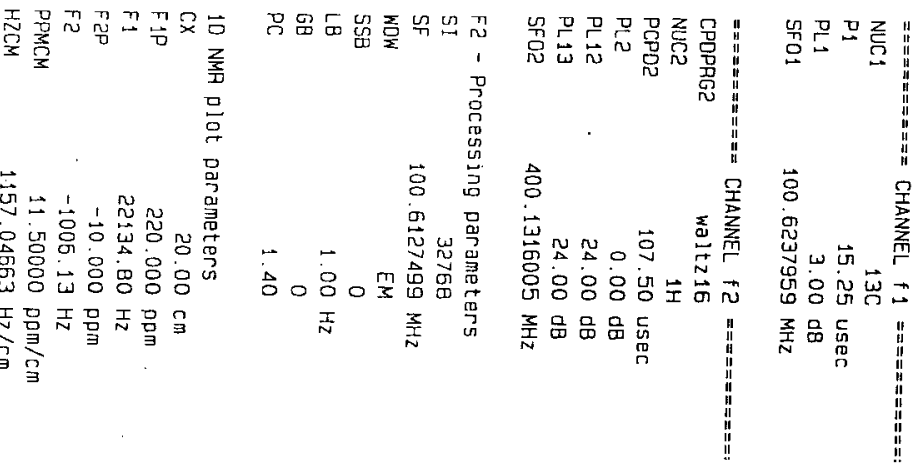

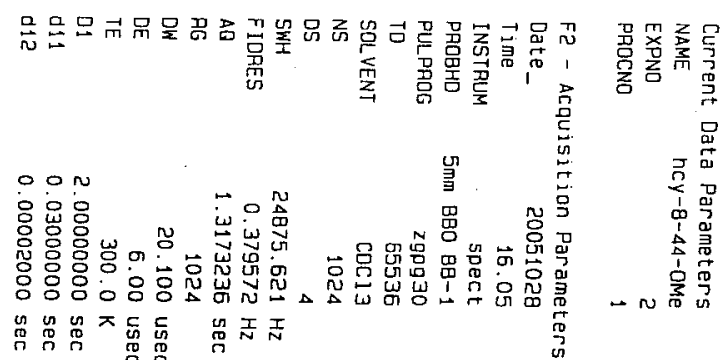



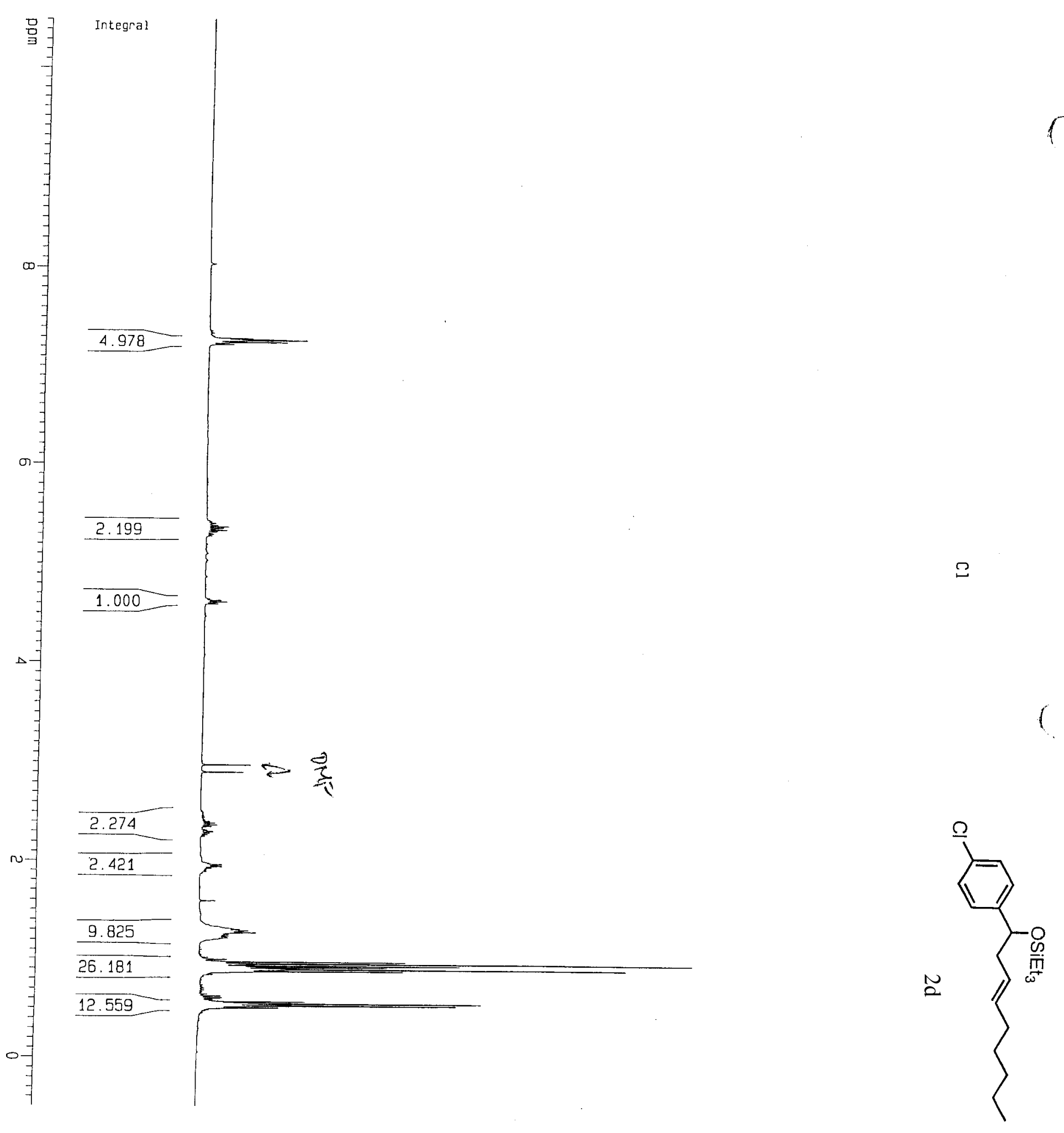

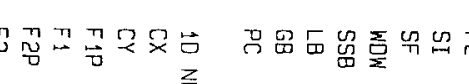

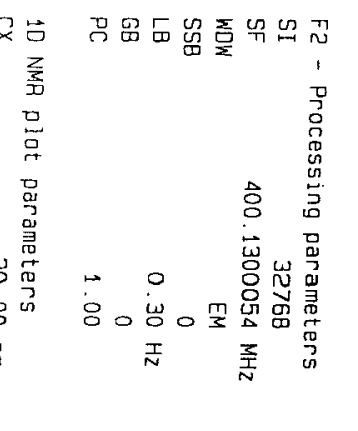

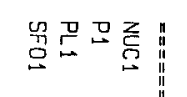

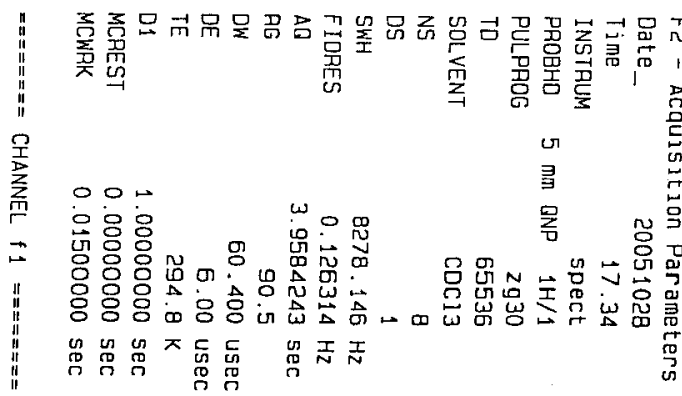

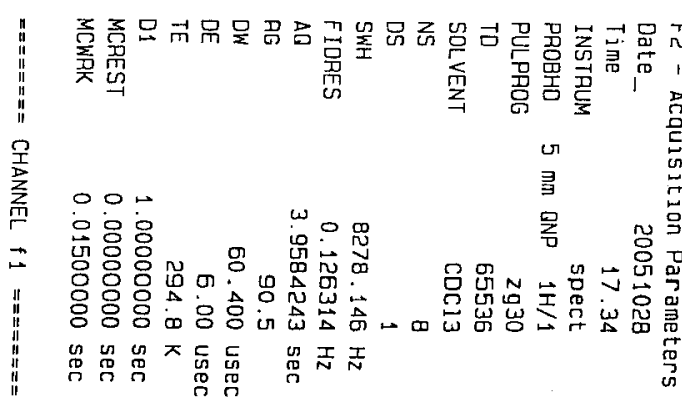

(2)

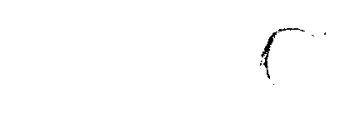

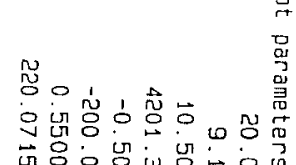

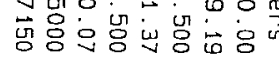

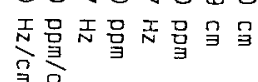

$\rightarrow$ 岕岕品岕

客安离宝

곳 品品

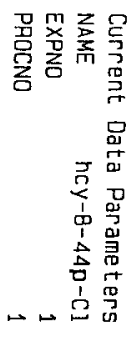




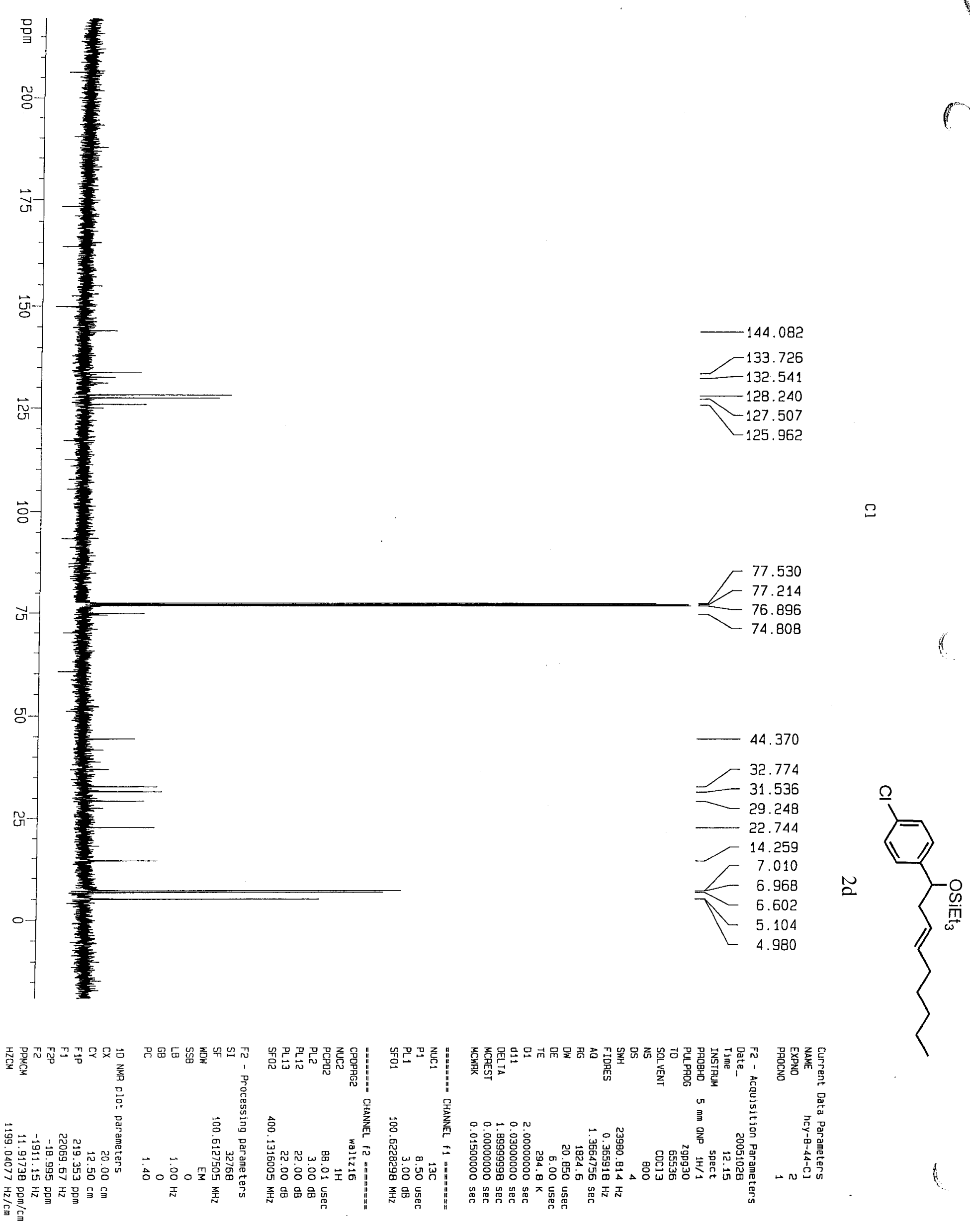




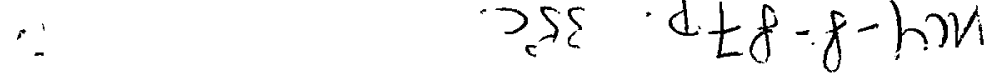

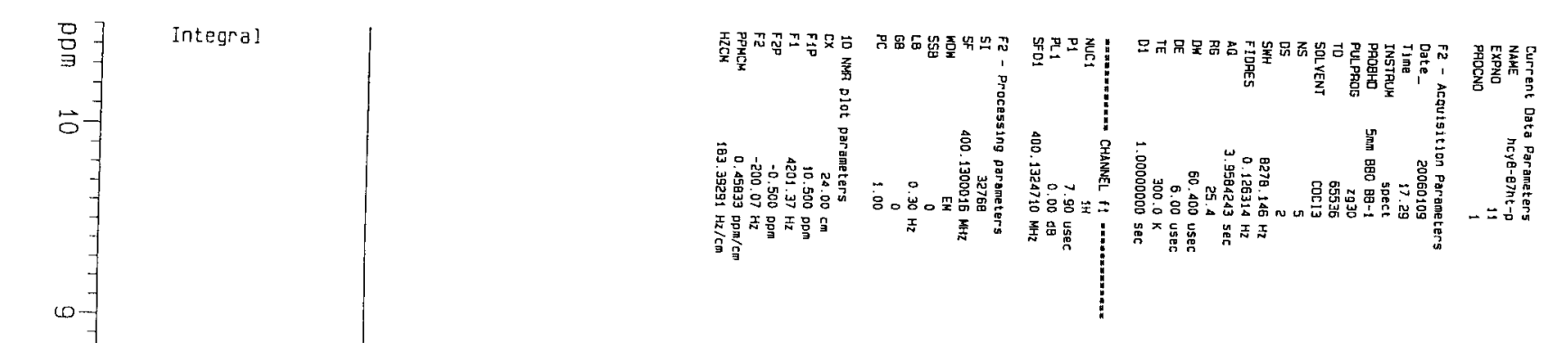

正 $\frac{\frac{3.147}{1.037}}{\frac{3.282}{3.28}}$
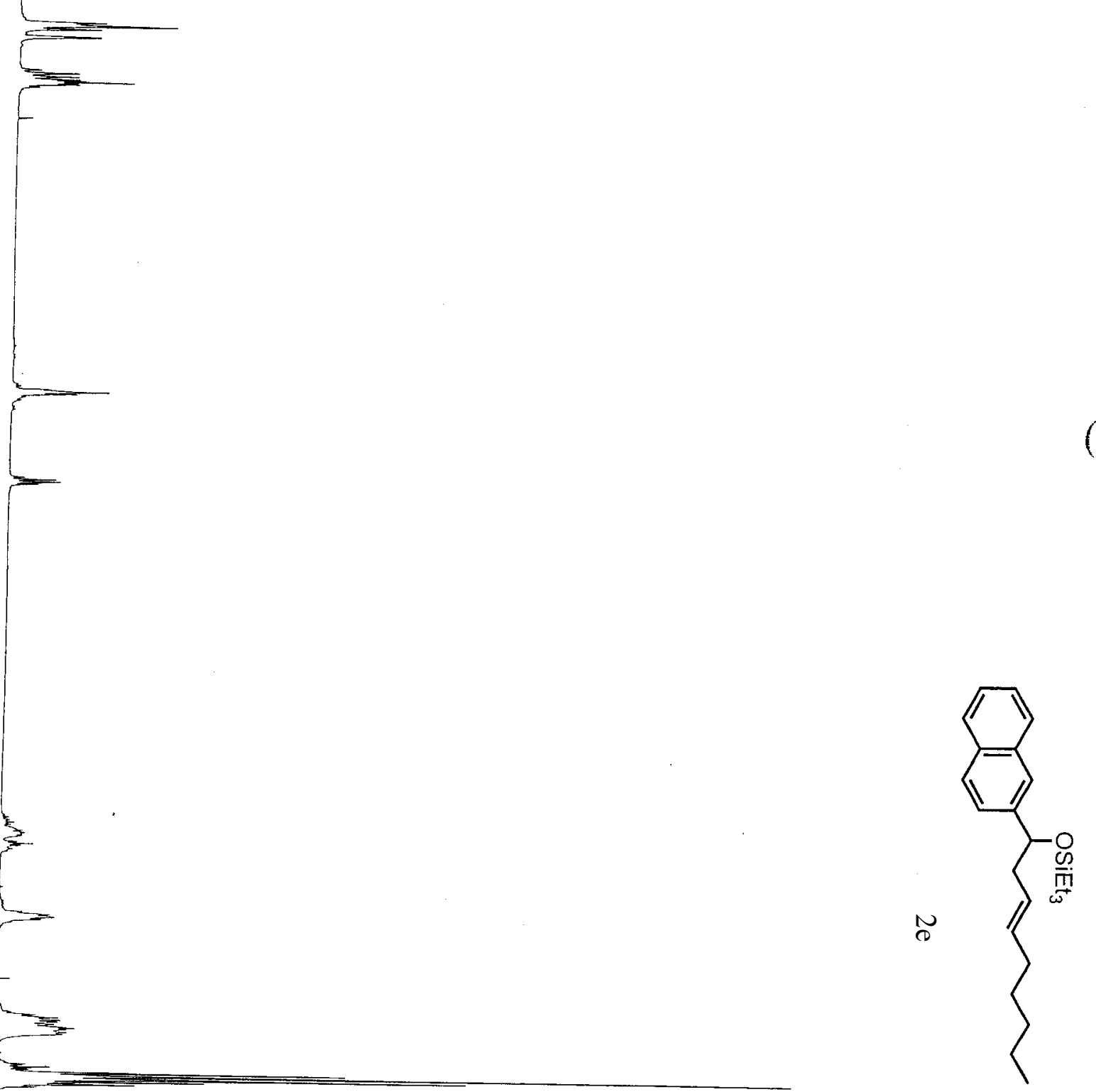


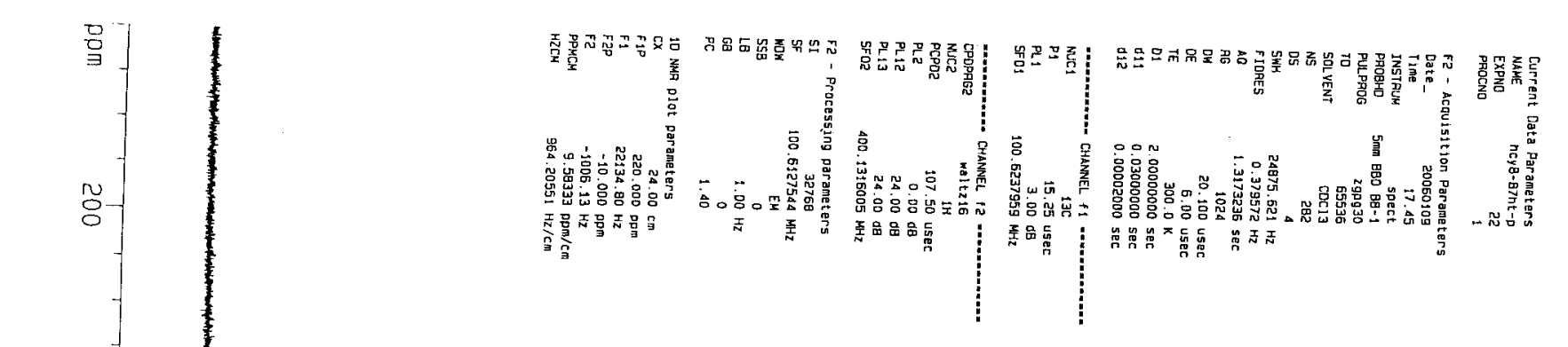

ppm
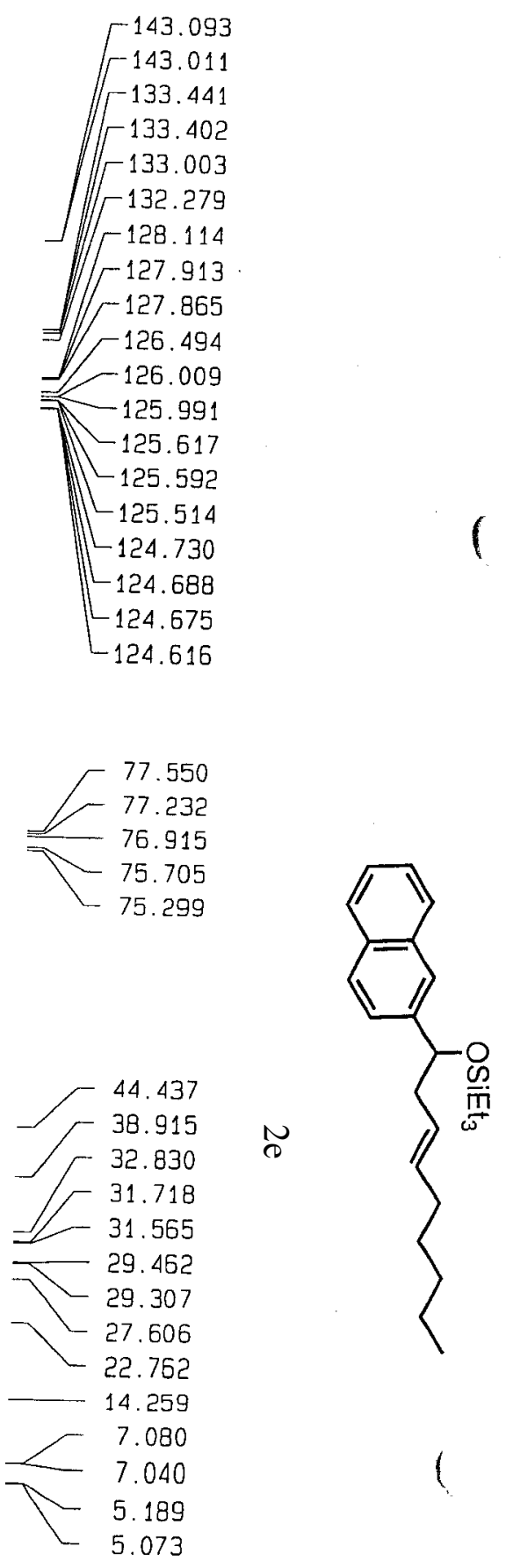

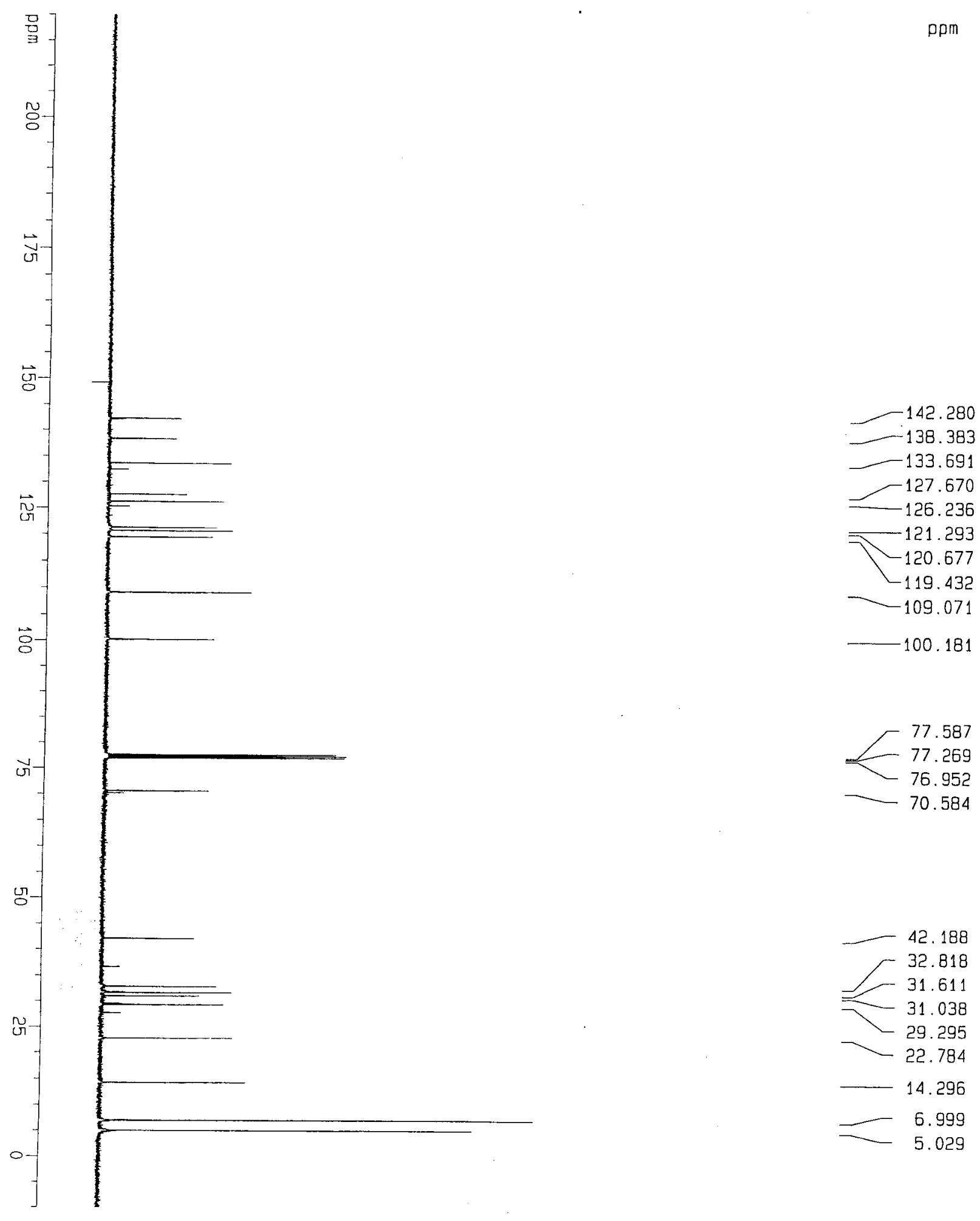

$\begin{array}{r}-29.295 \\ -22.784 \\ \hline\end{array}$

14.296

- 6.999

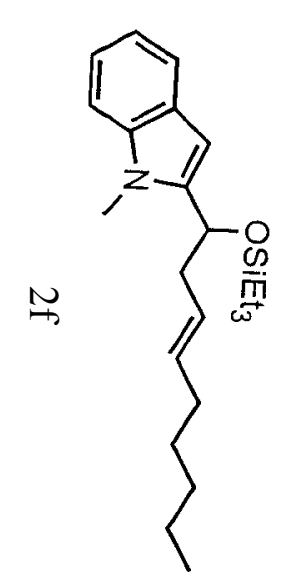

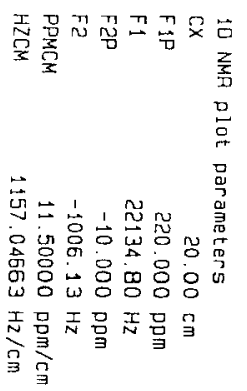

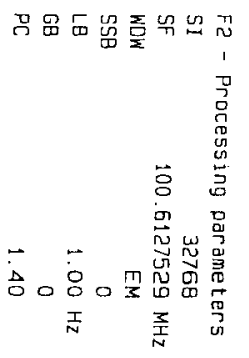

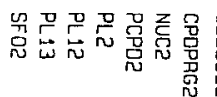

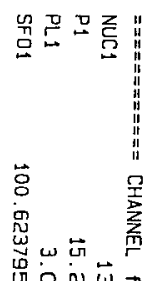

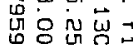

等点总

5.029

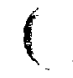

$-\quad 5.029$

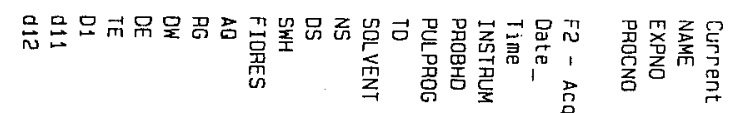

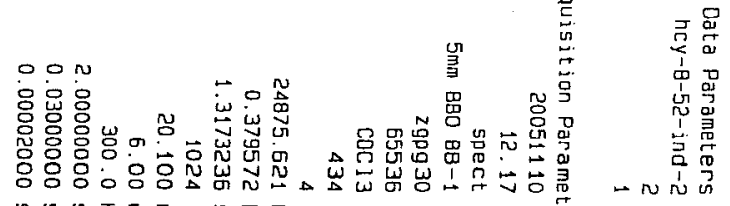

(n) 


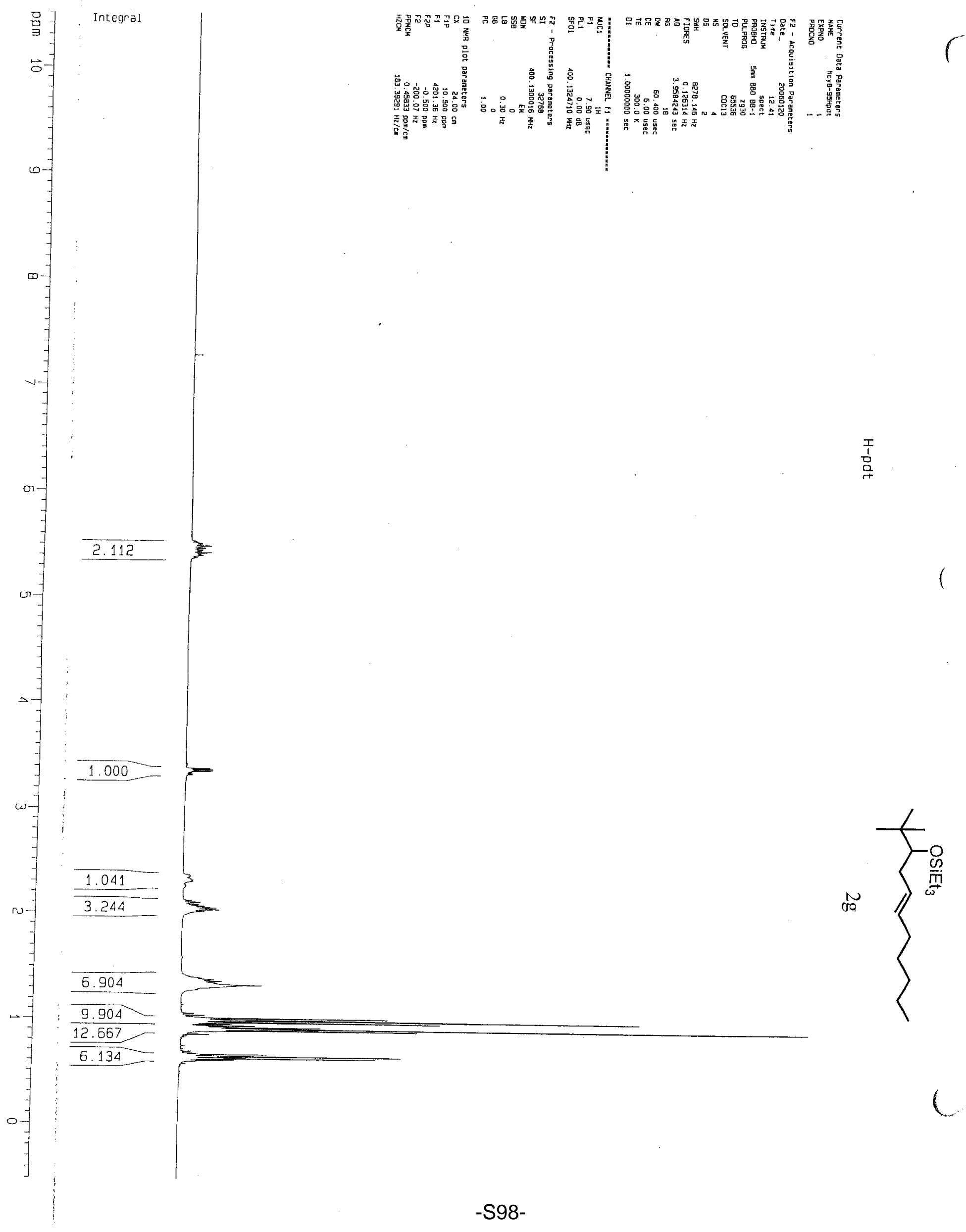



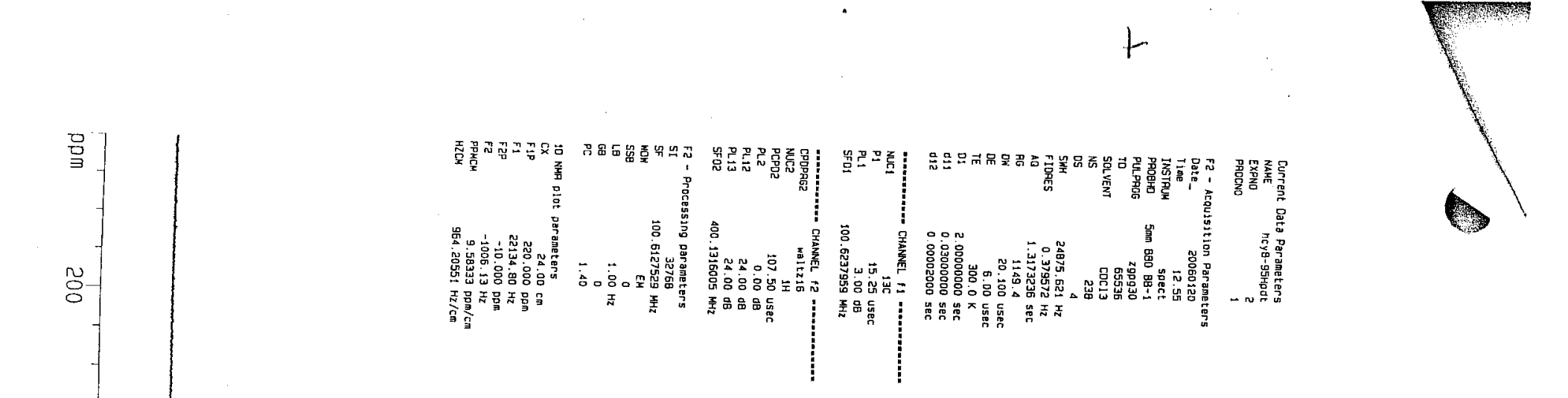

ppm

品

항-

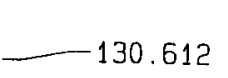

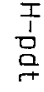

范

突

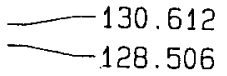

$-128.506$

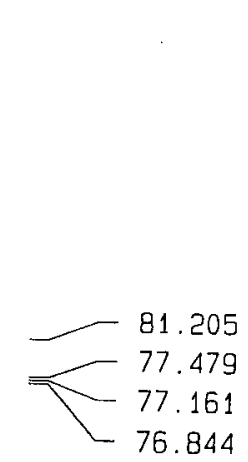

\%

s.

$$
\text { 용 }
$$
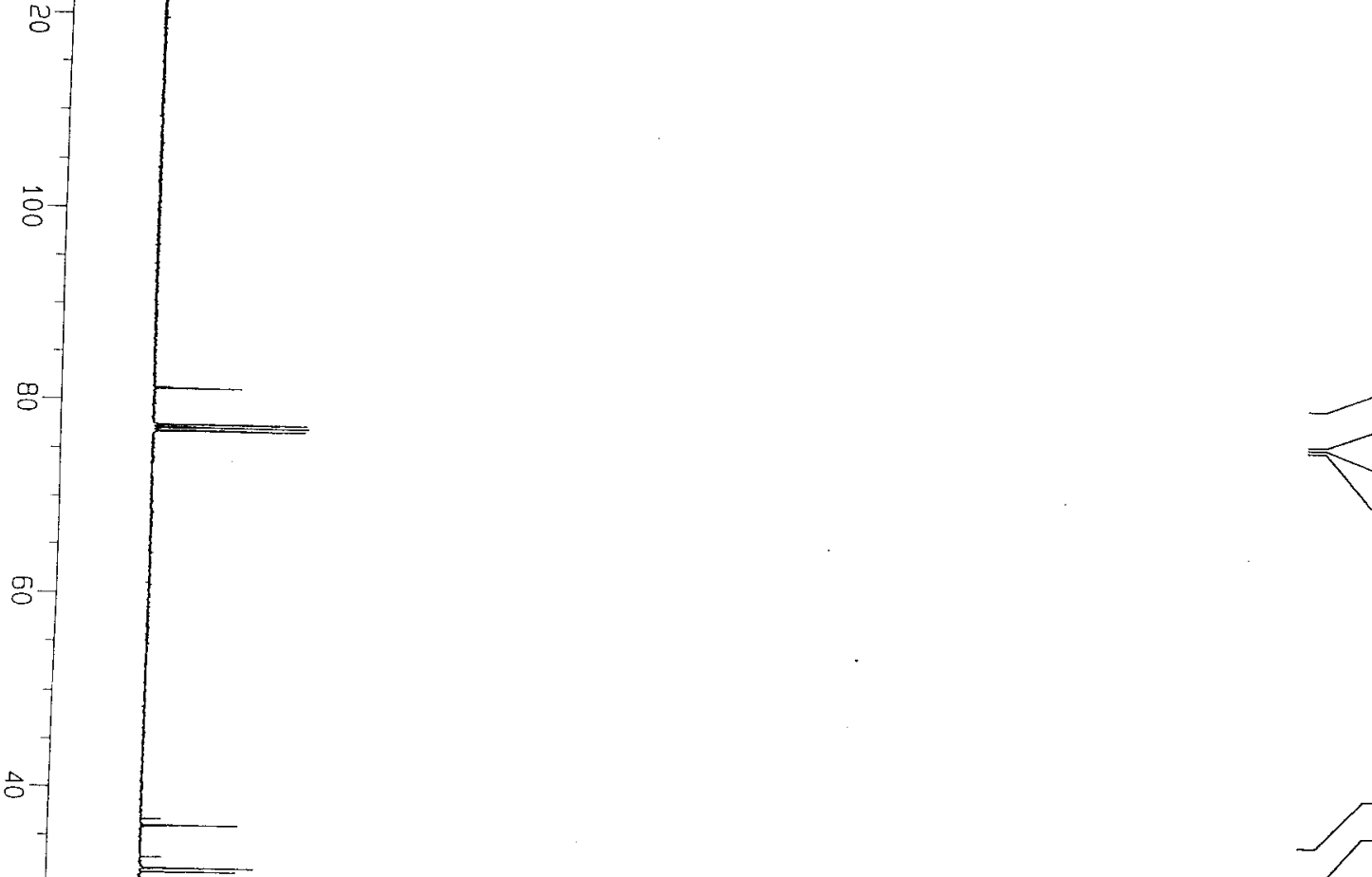

36.155

31.793

31.355

29.463

$-27.601$

$-26.480$

- 22.775

14. 241

$-\quad 7.293$

5.693

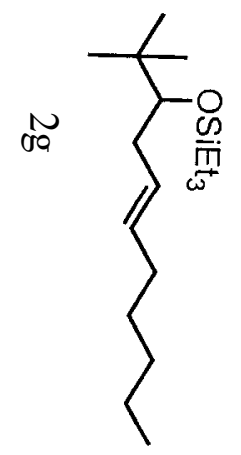

-S99- 


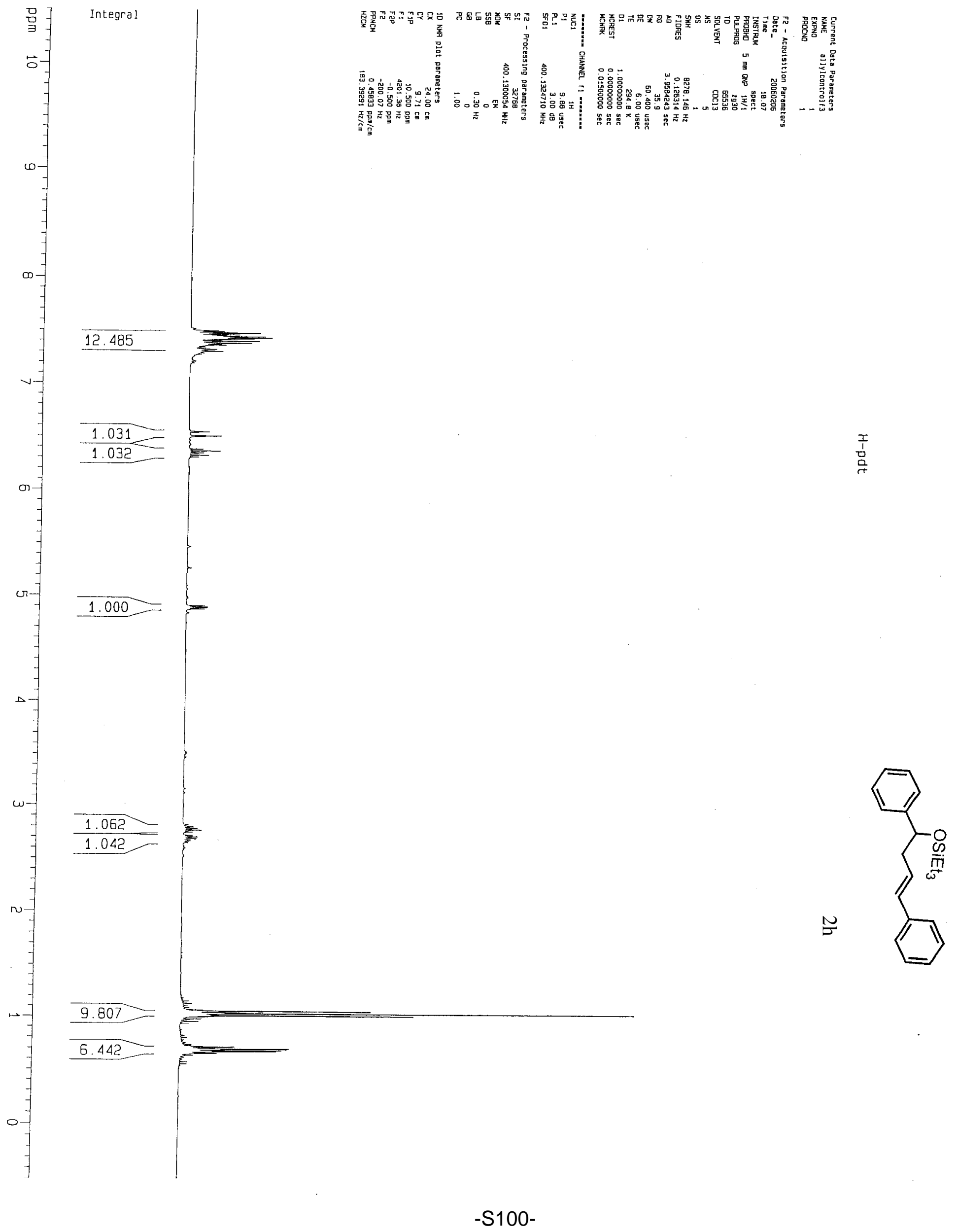




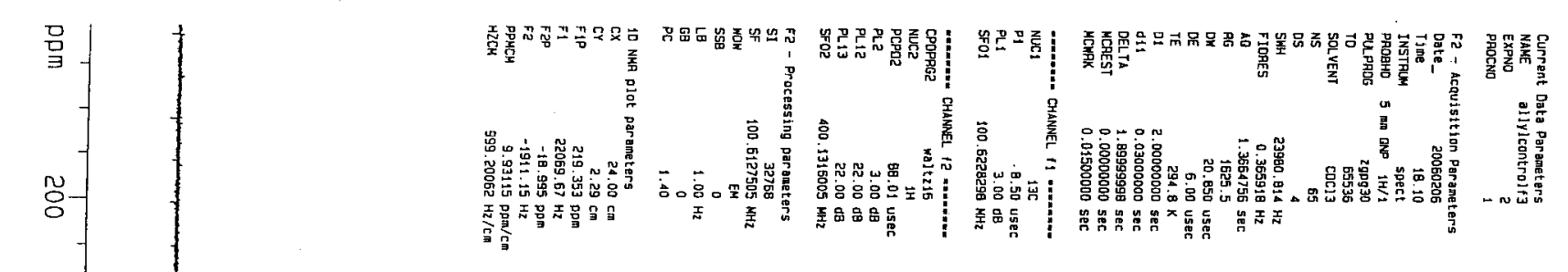

ppm
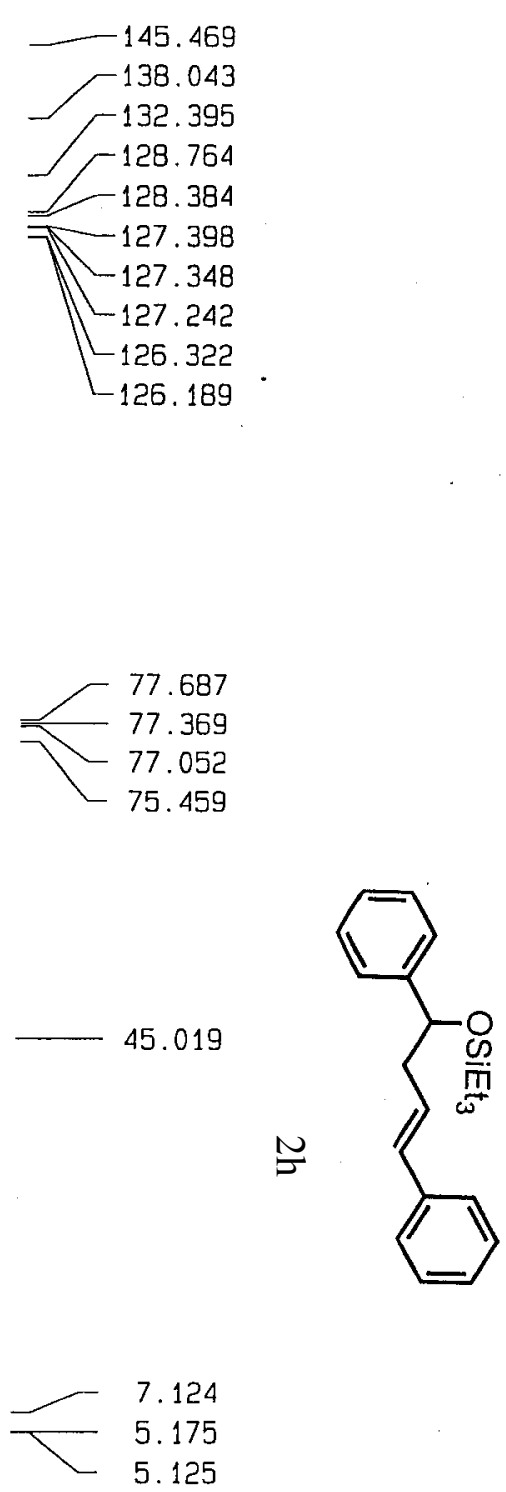


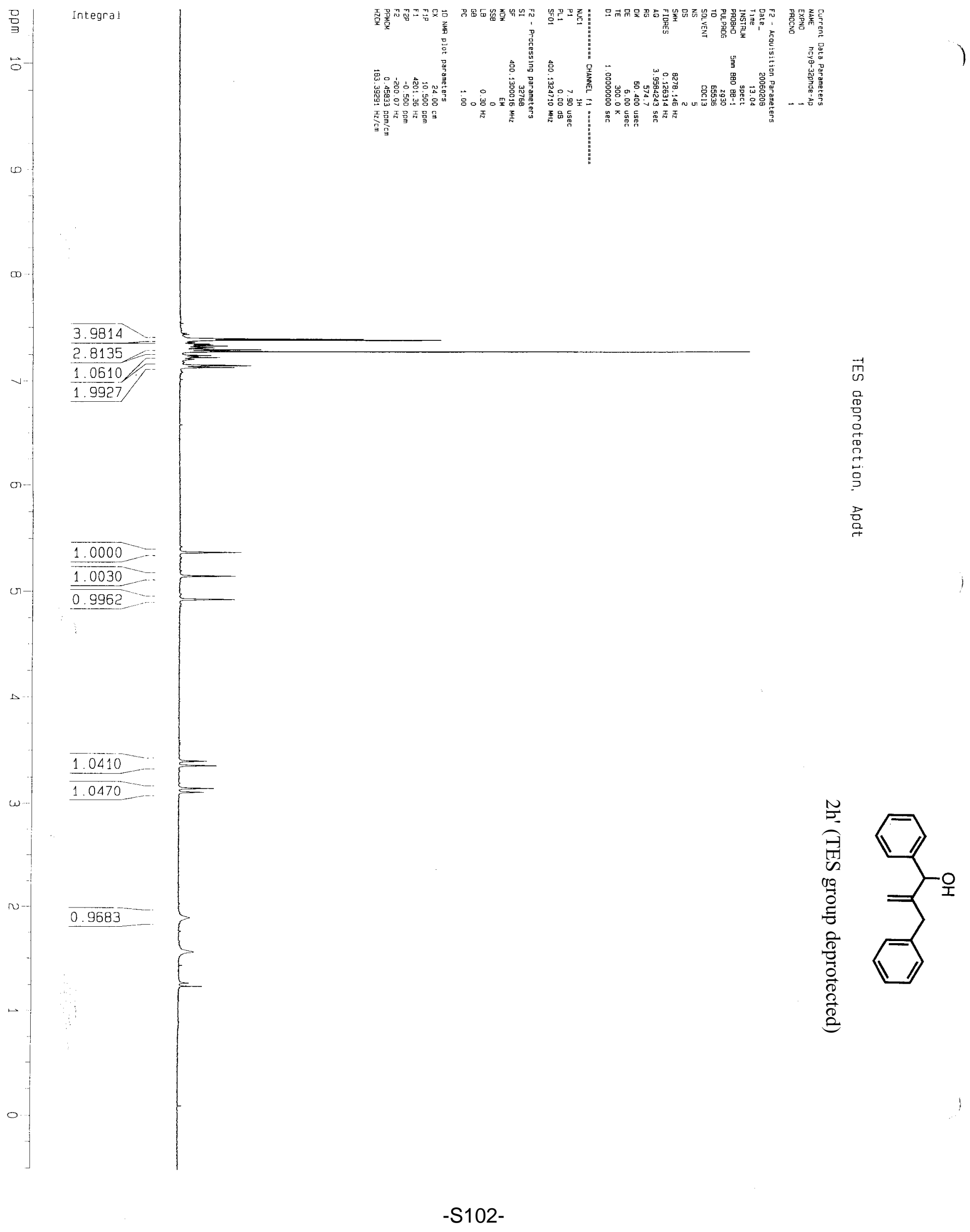




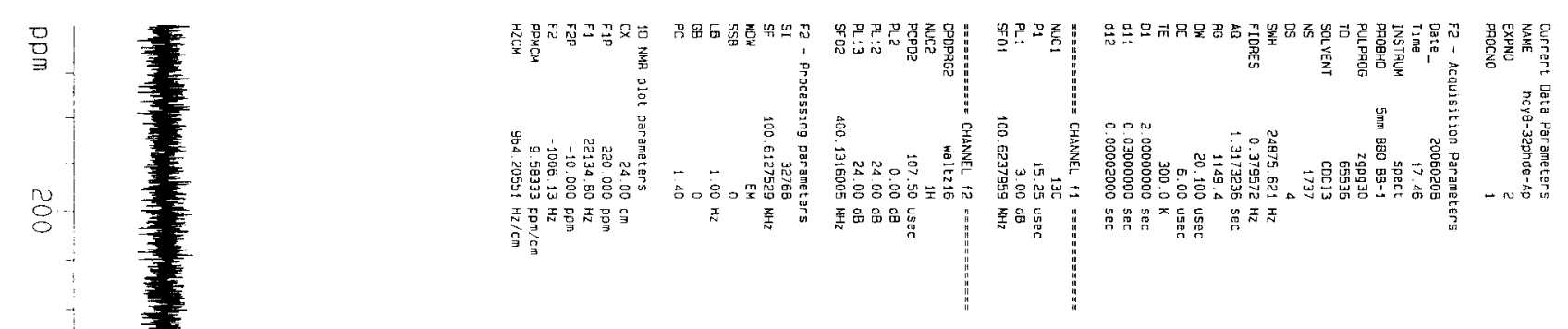

ppm

$\stackrel{\circ}{\circ}$

$\vec{\sigma}$

$\stackrel{\vec{b}}{b}$

$-150.629$

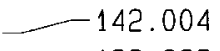

$-139.296$

$-129.367$

$-128.696$

$-128.542$

$\stackrel{\leftrightarrow}{\circ}$

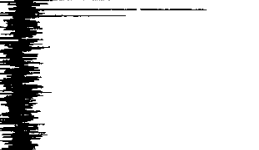

$\overrightarrow{8}$

$\infty$

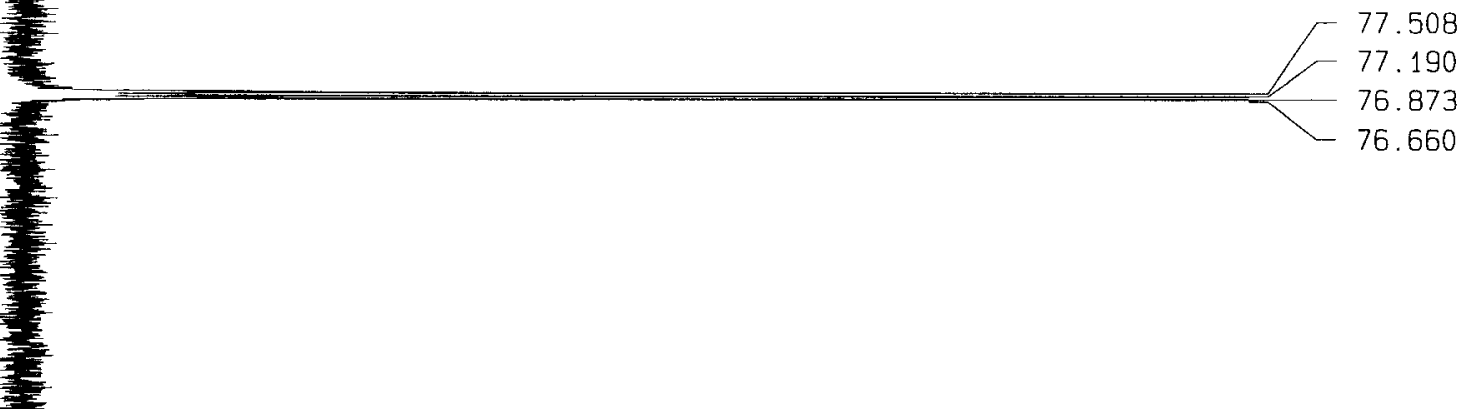

39.196

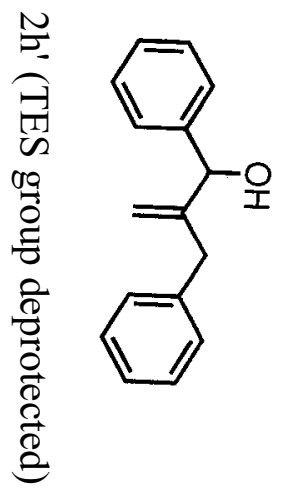




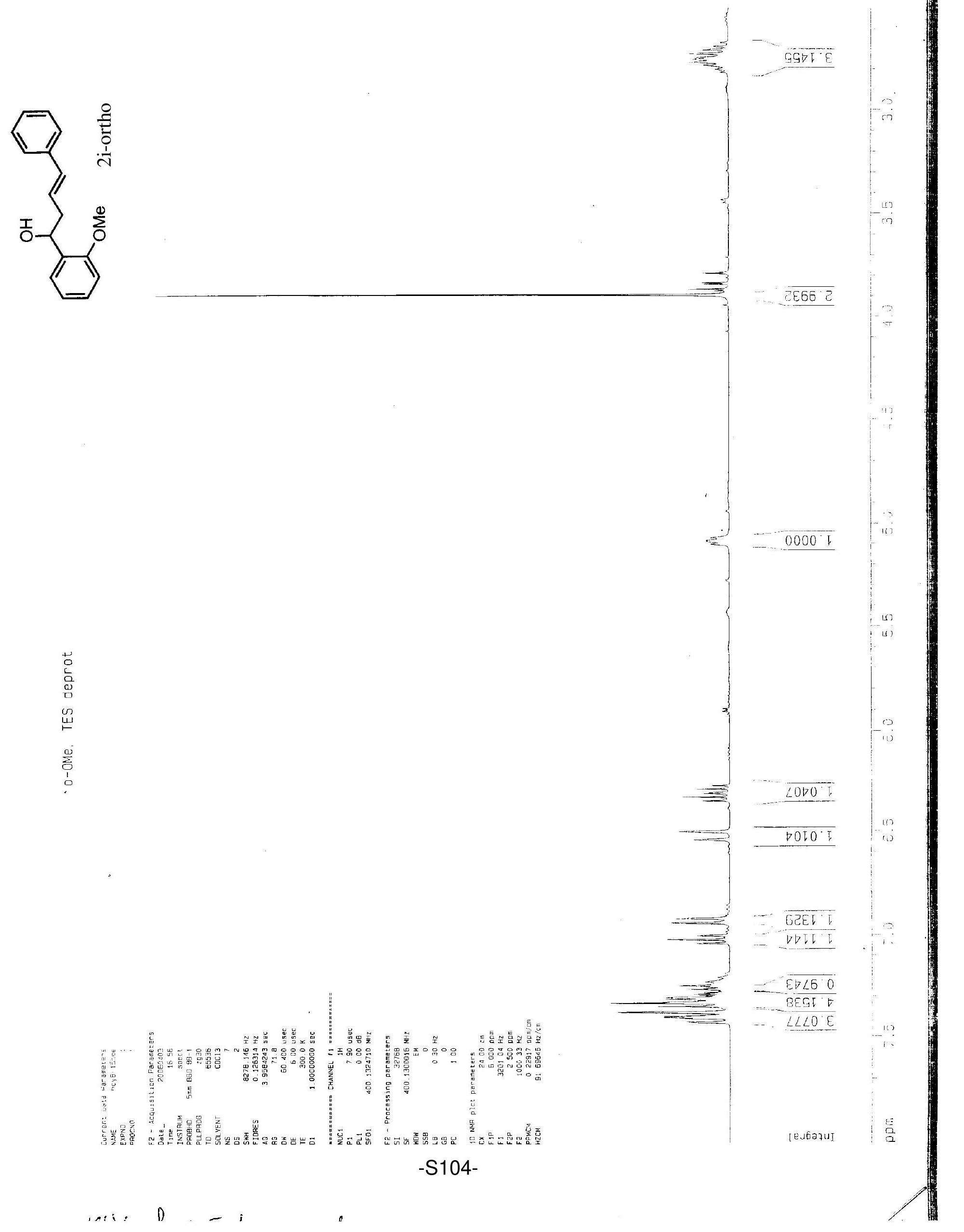



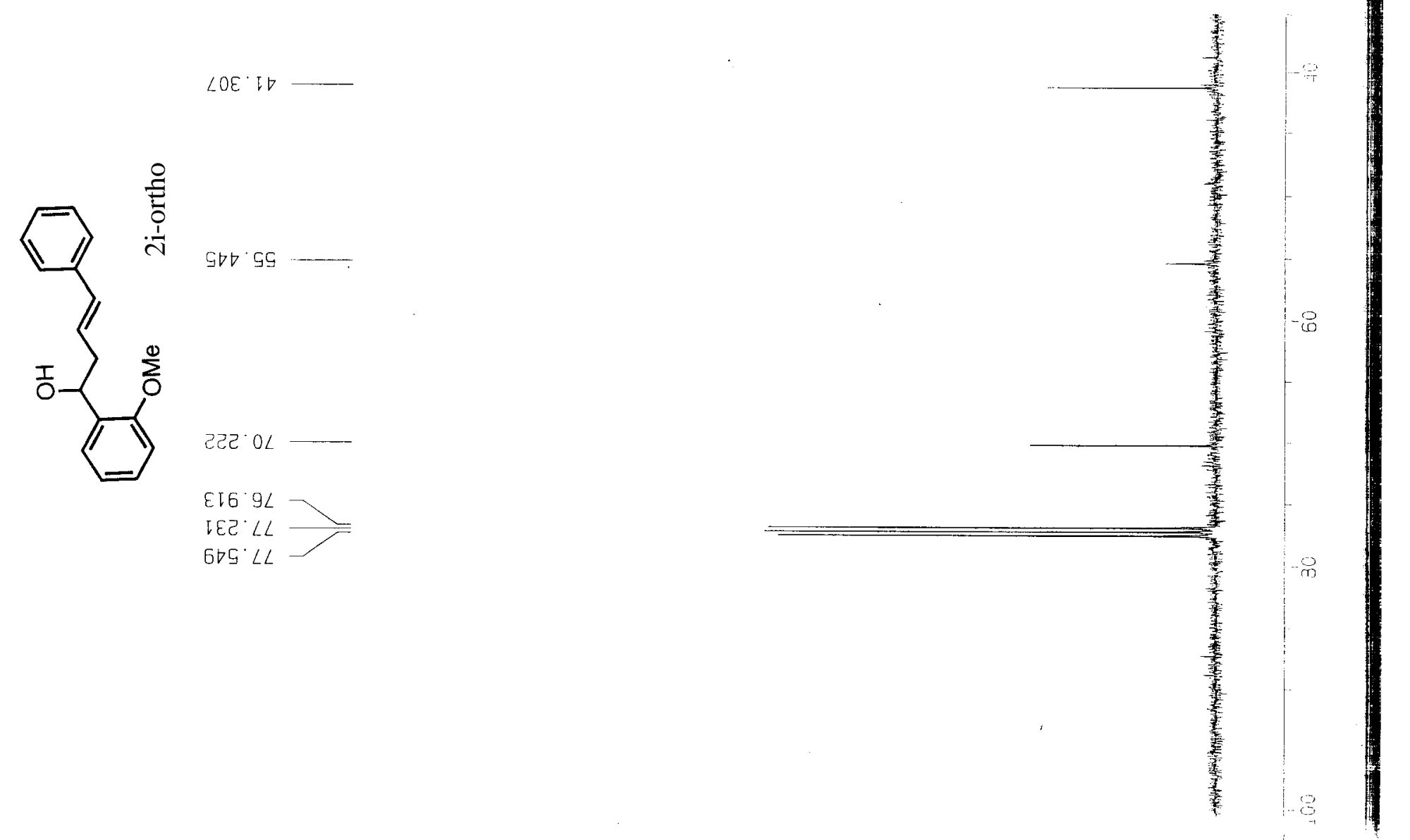

989.0⿰丿⿺丄丶万 -
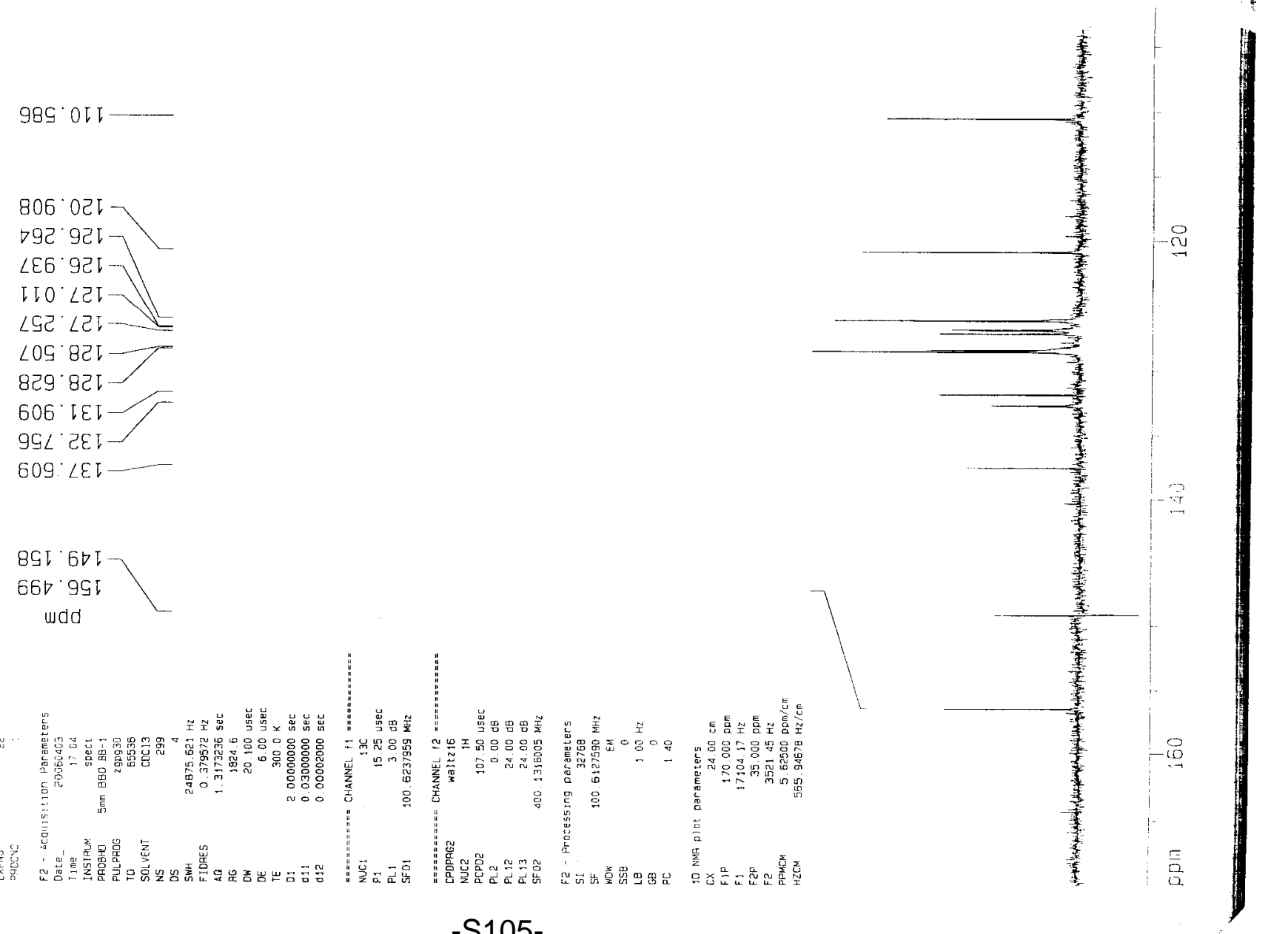


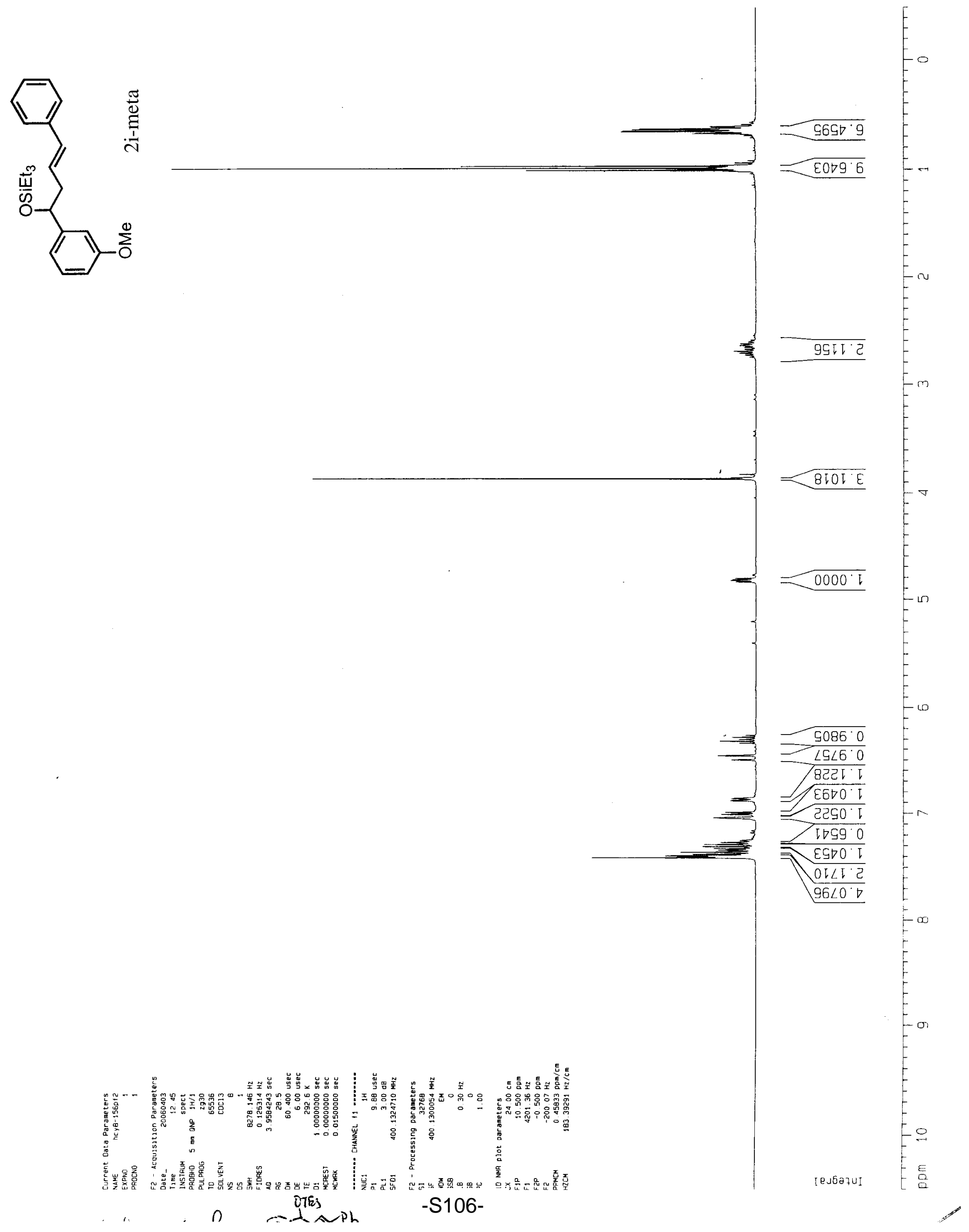




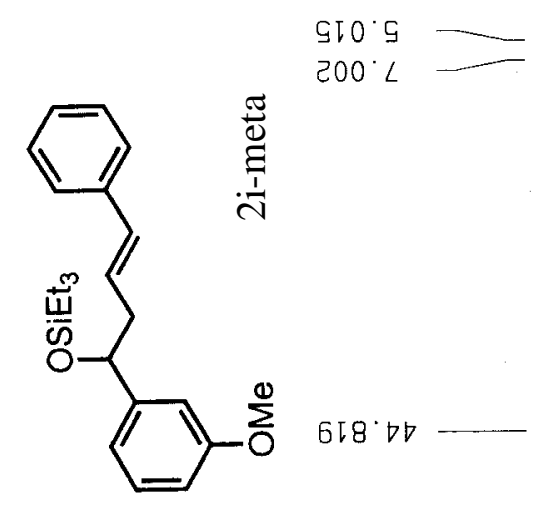

โ8ट $99-$
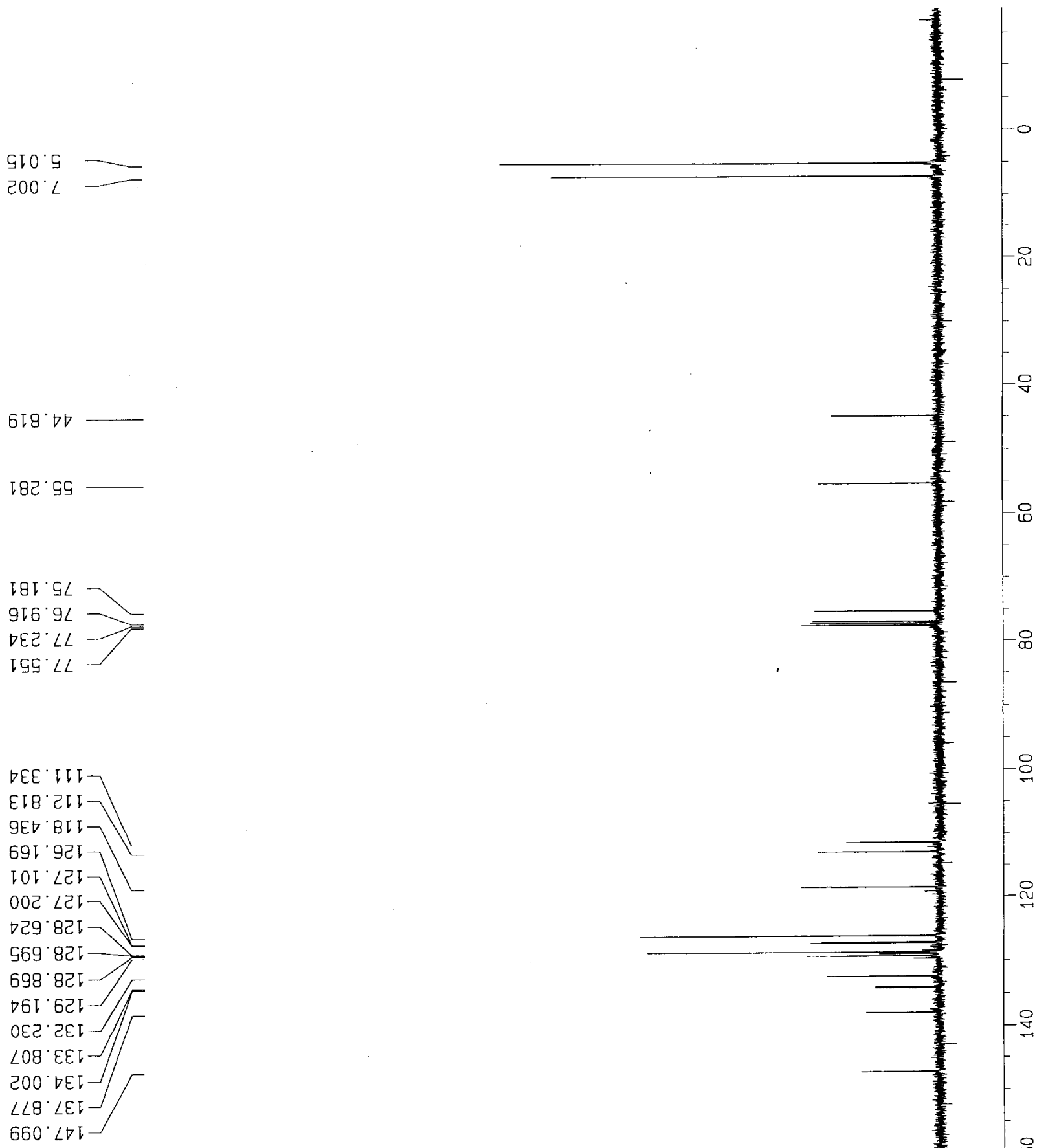

$\angle \angle 9^{\circ} 69^{\circ}$

udd

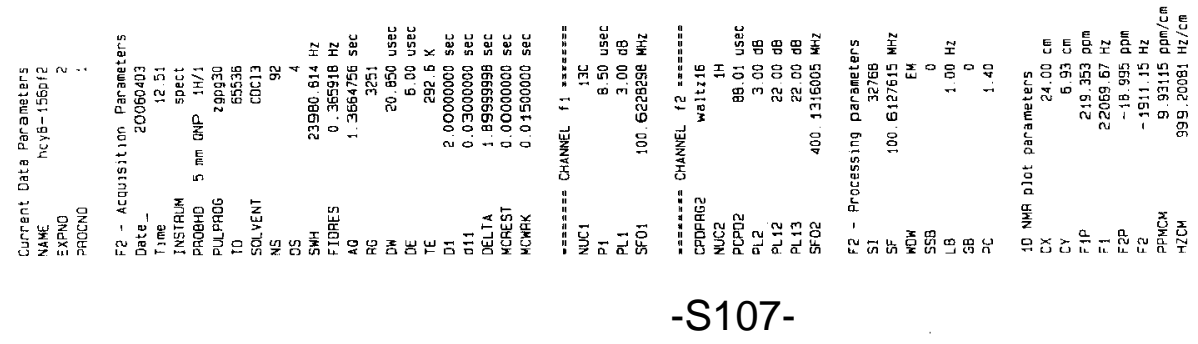




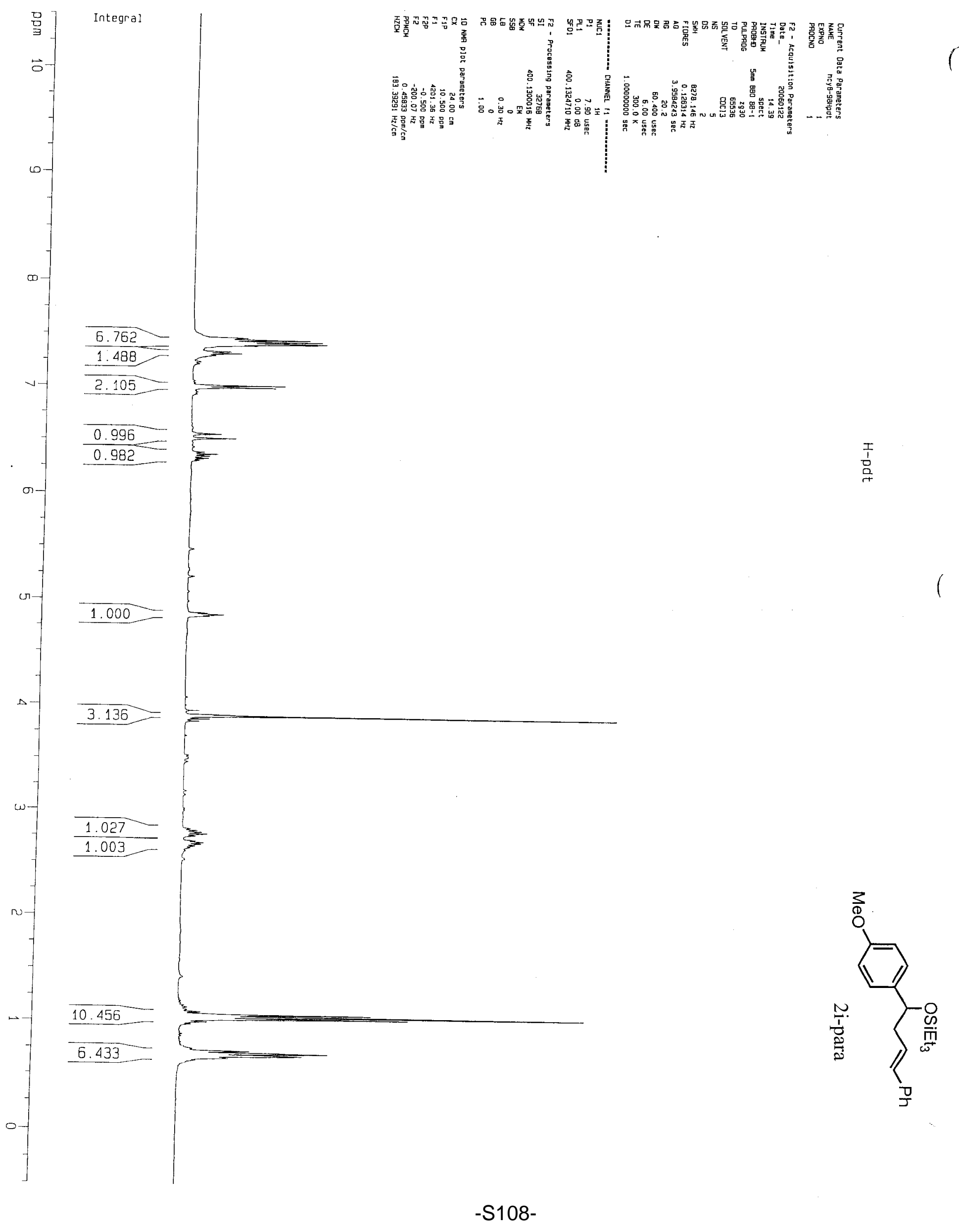



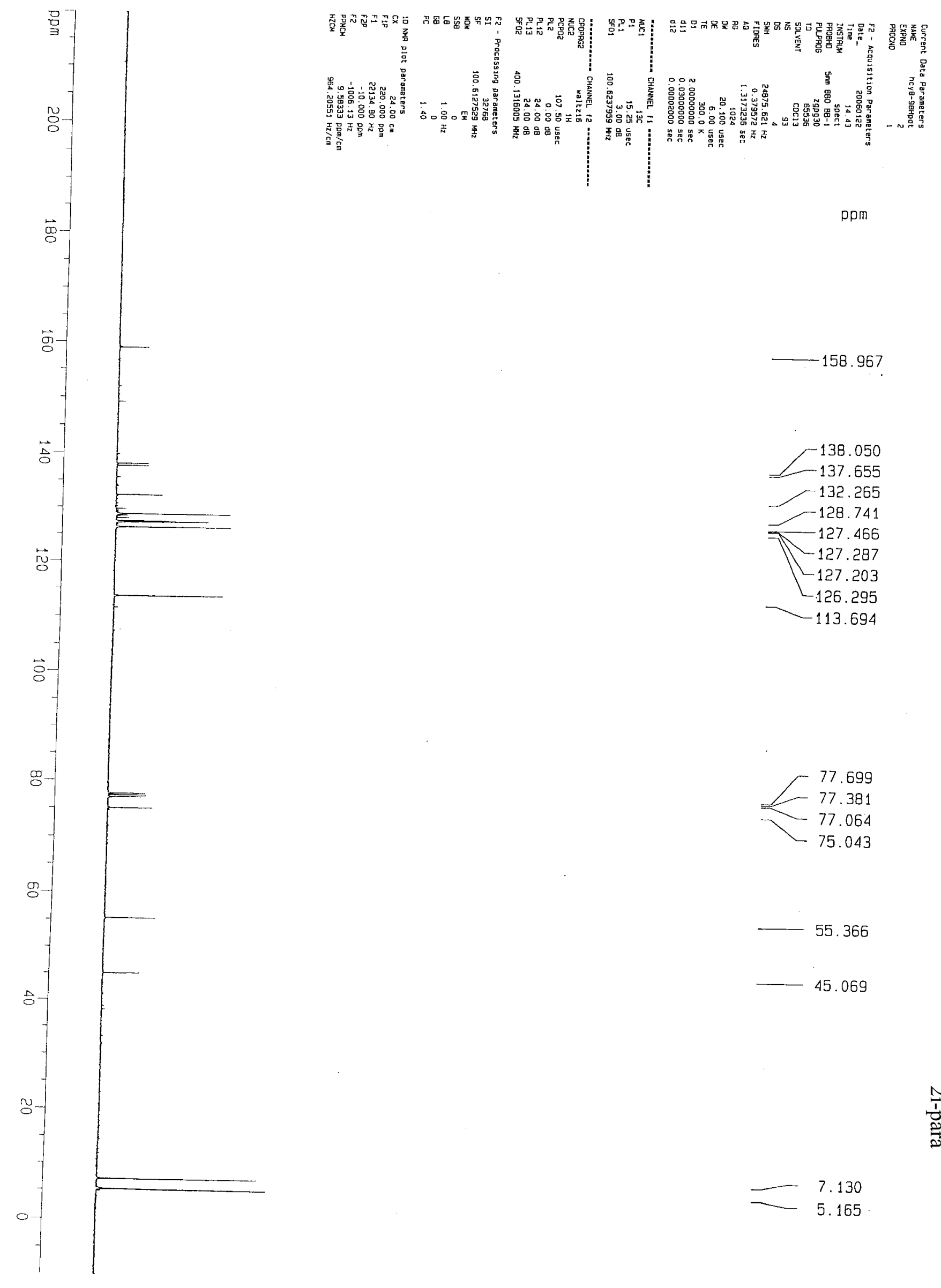

ppm
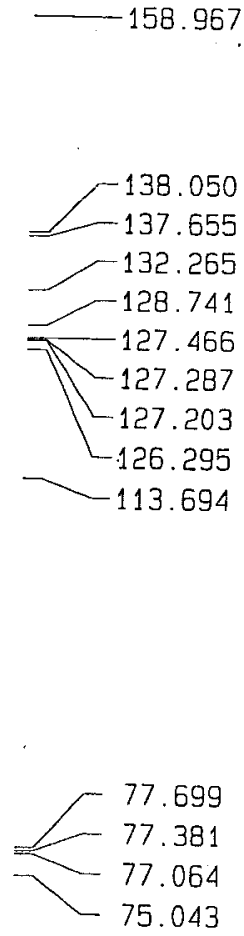

55.366

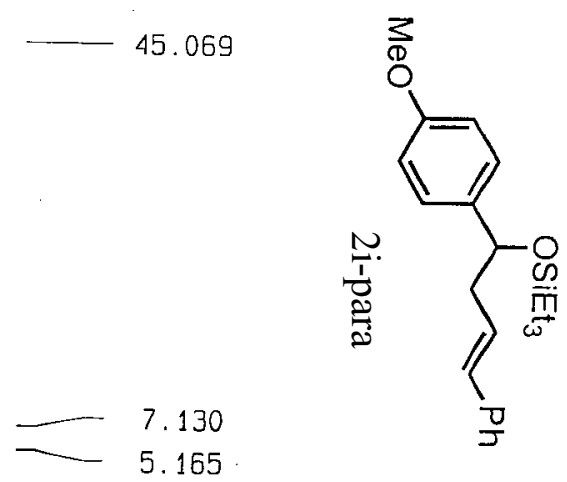




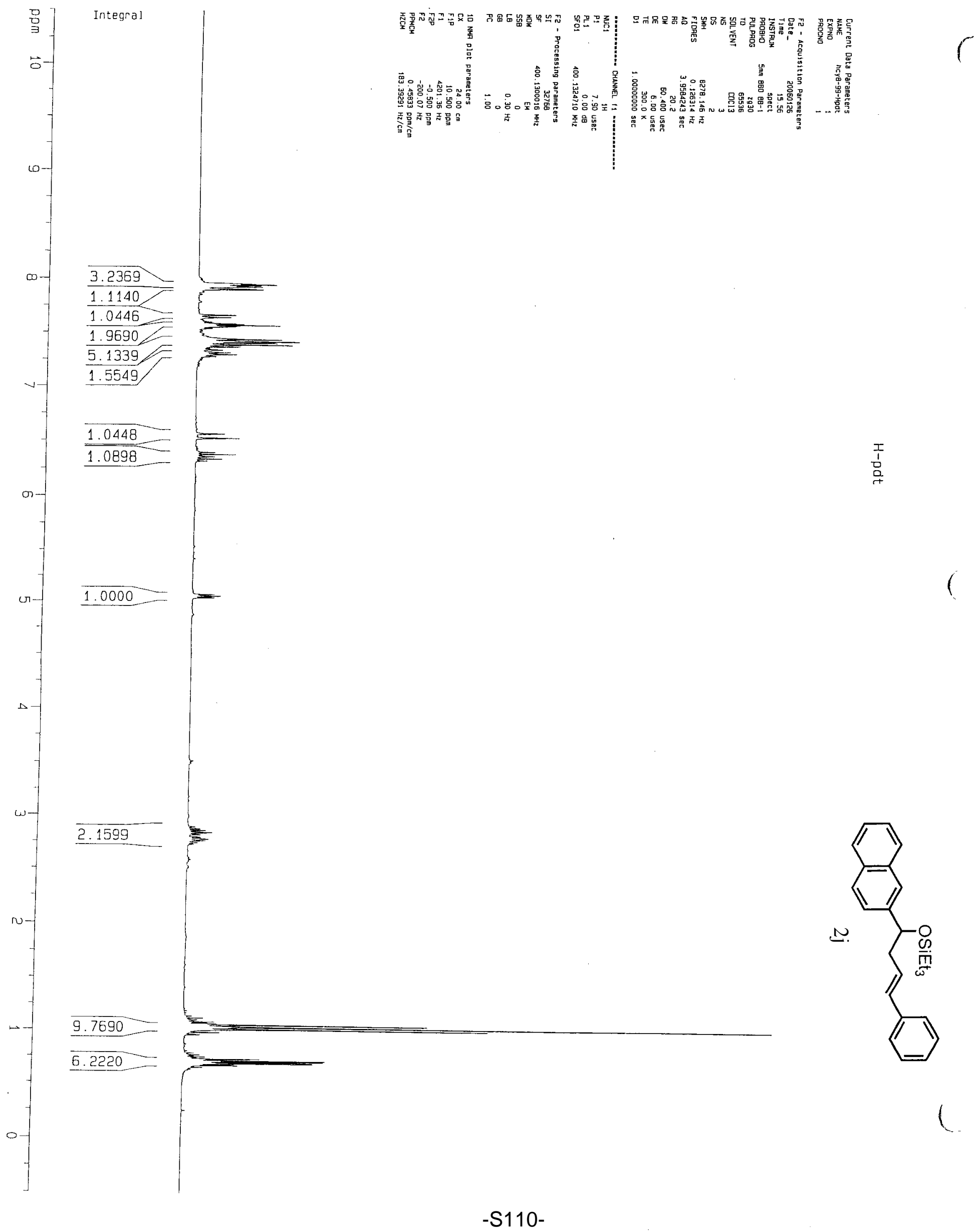




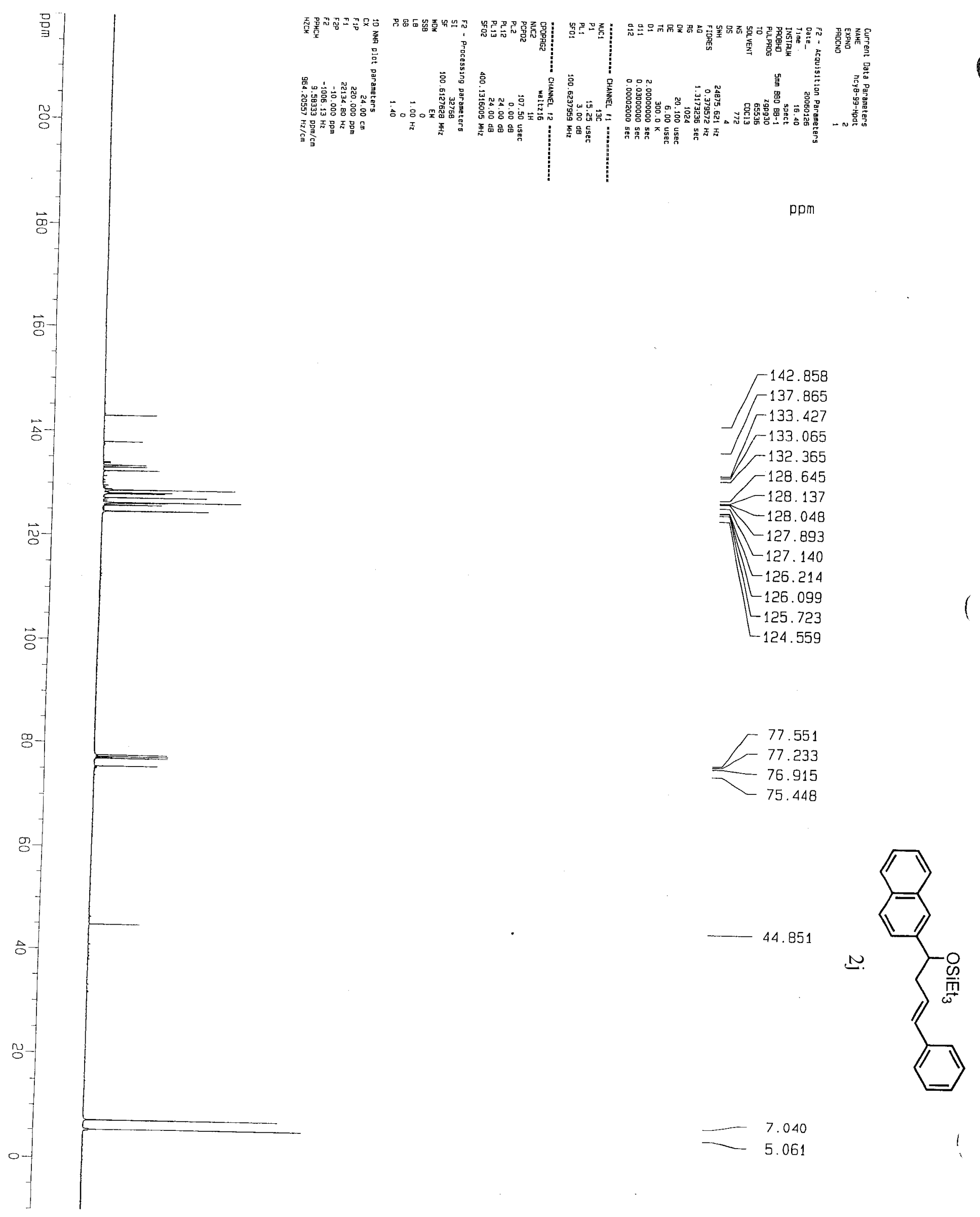




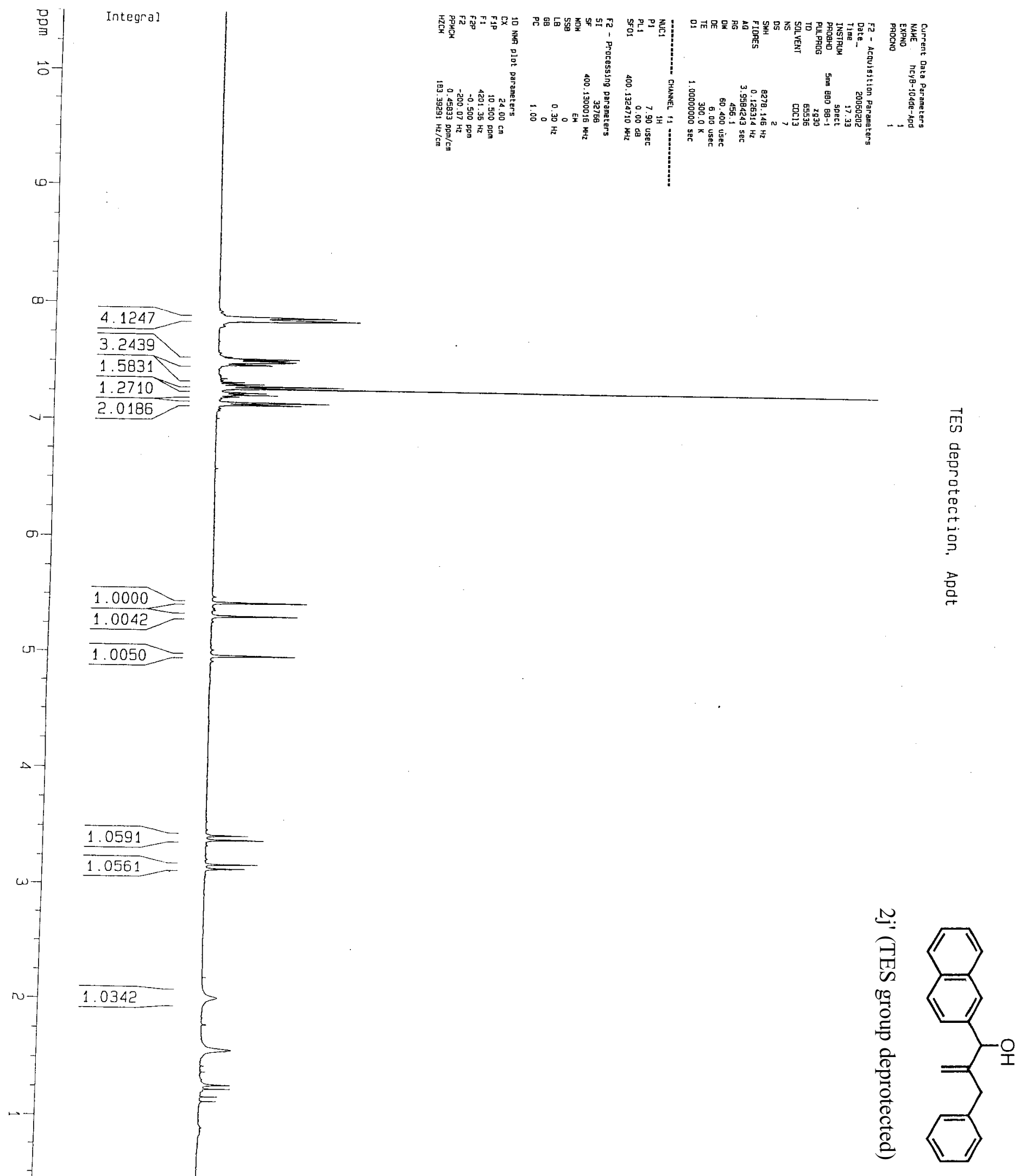

-S112- 


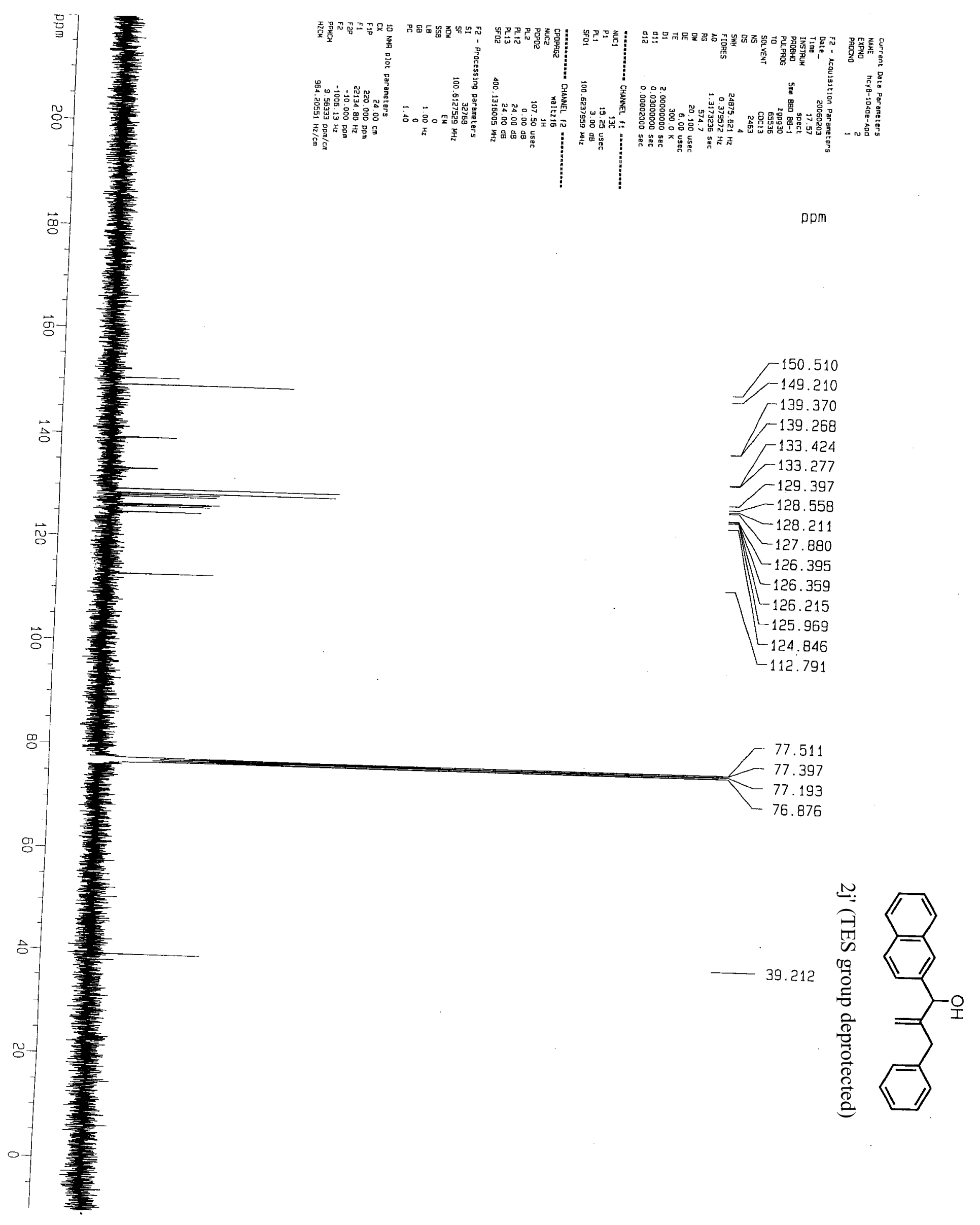




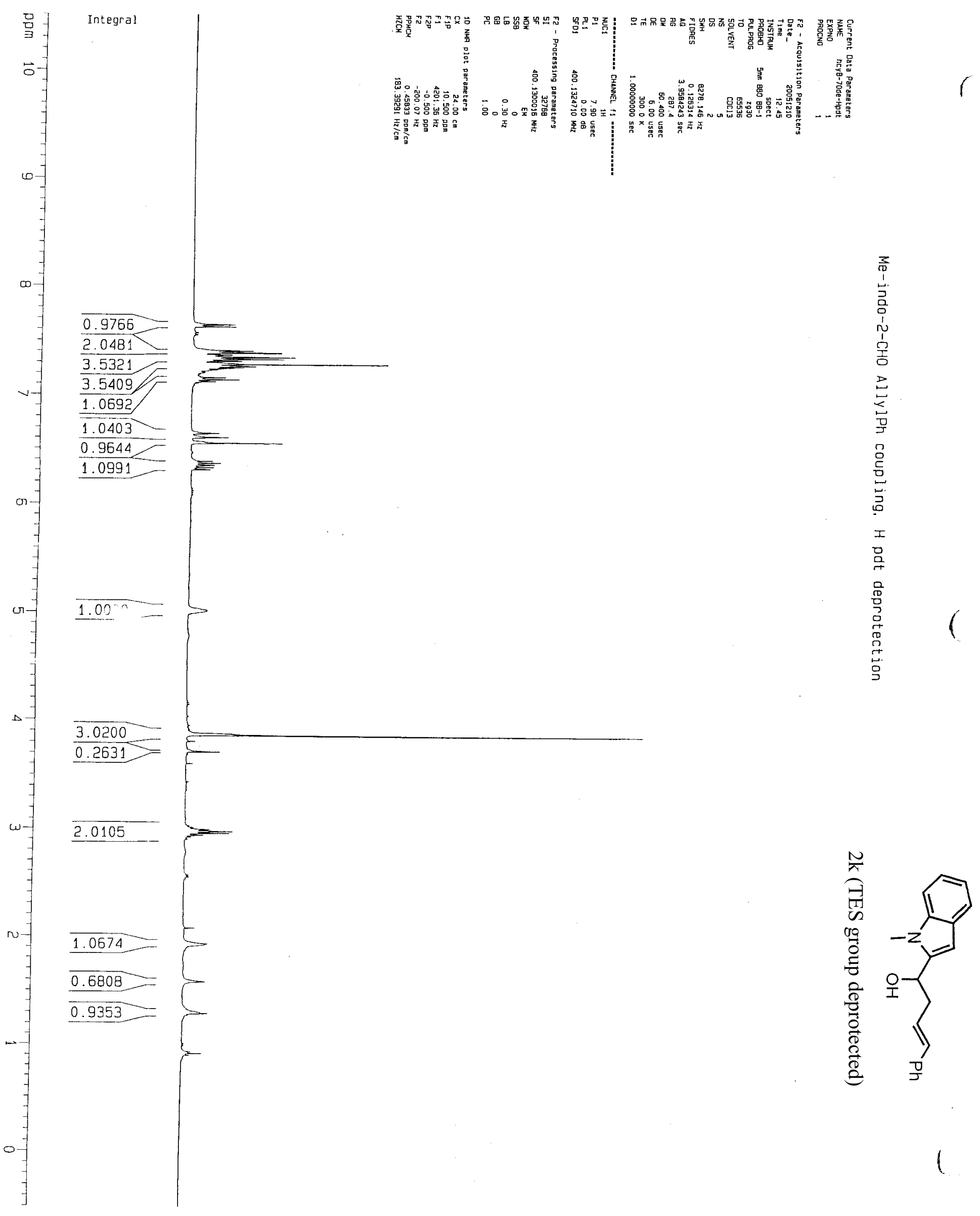




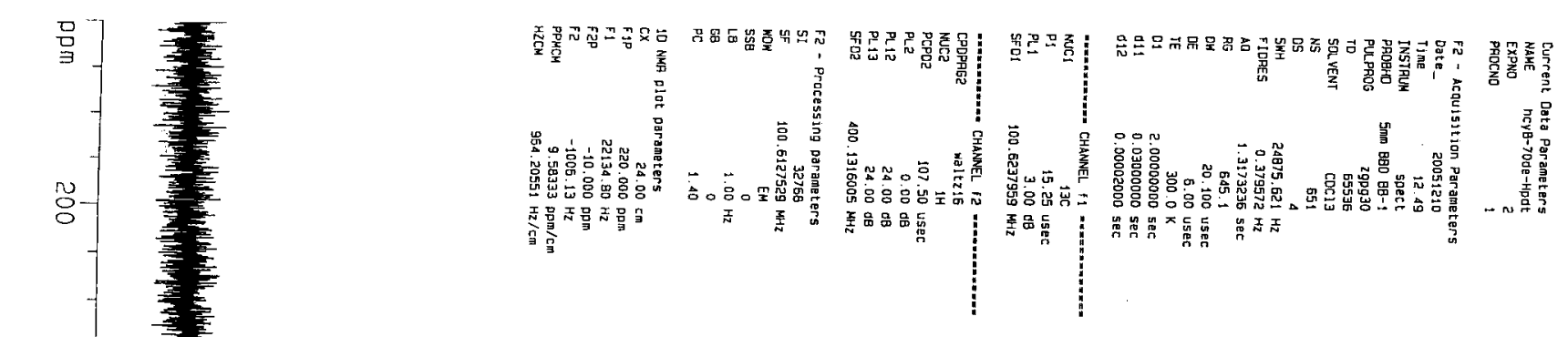

ppm

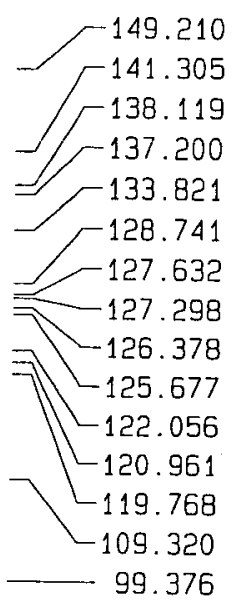

\&

횽

点

$\ddot{\tilde{O}}$

s

$\stackrel{\leftrightarrow}{\mathrm{D}}$

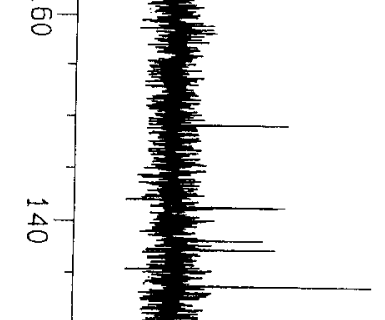

$\overrightarrow{8}-$
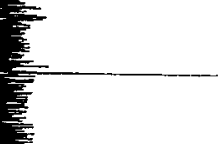

ב-

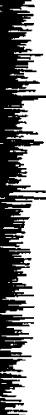
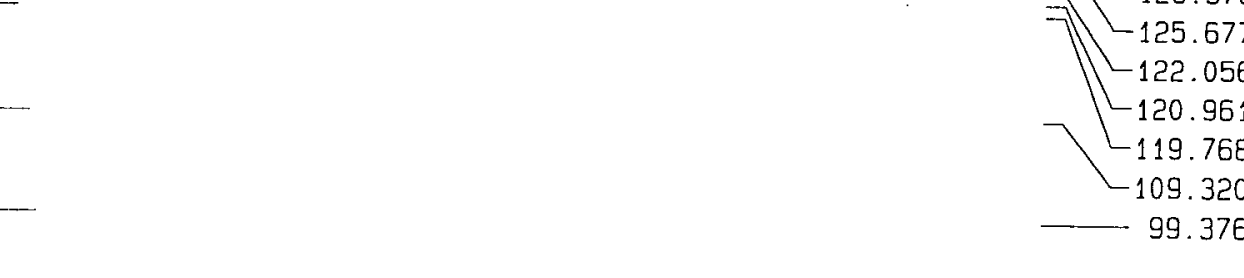


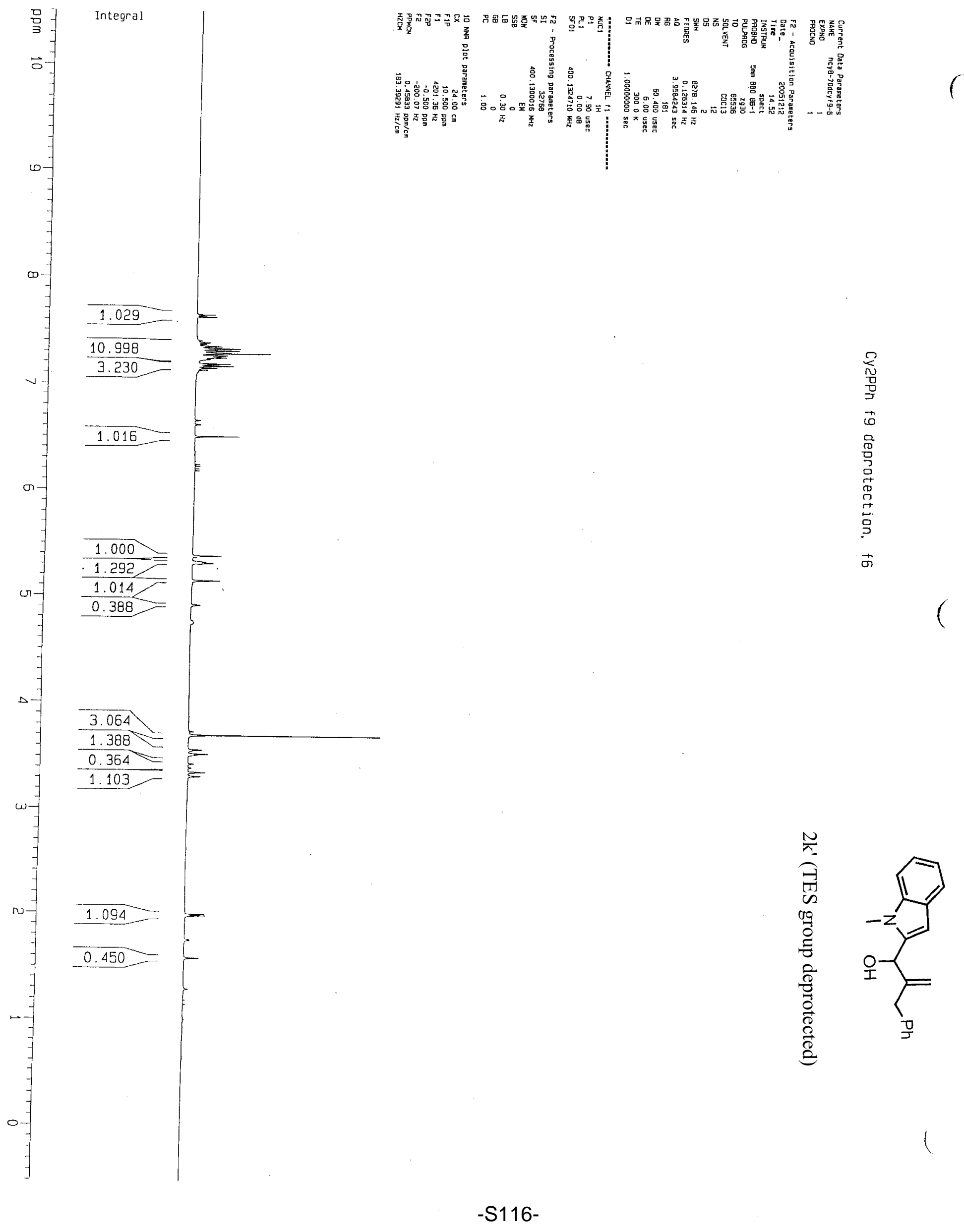



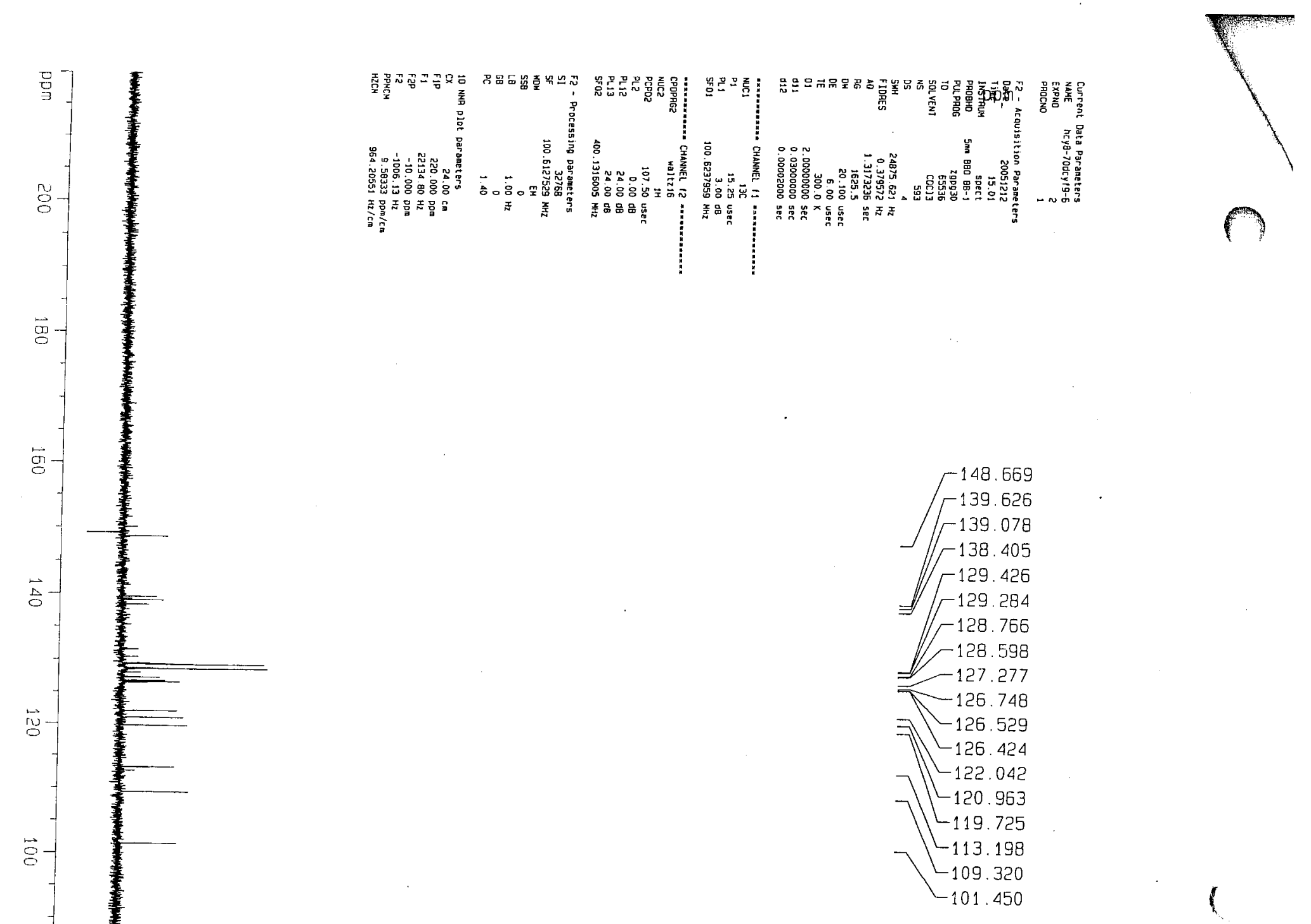

몽

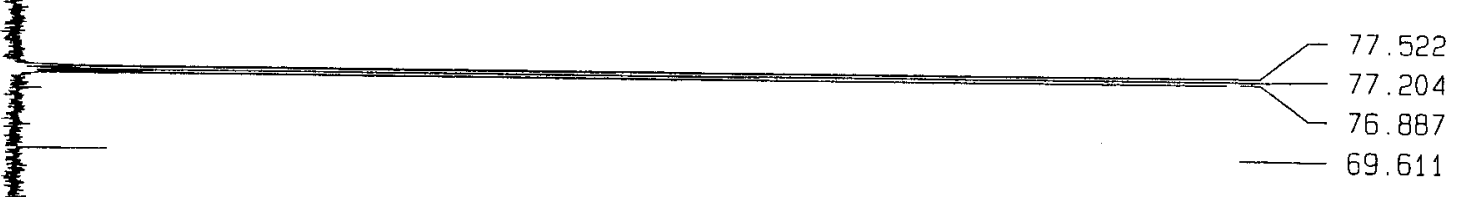

है

40.225

30.292

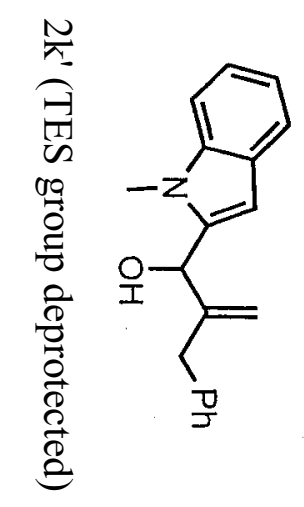




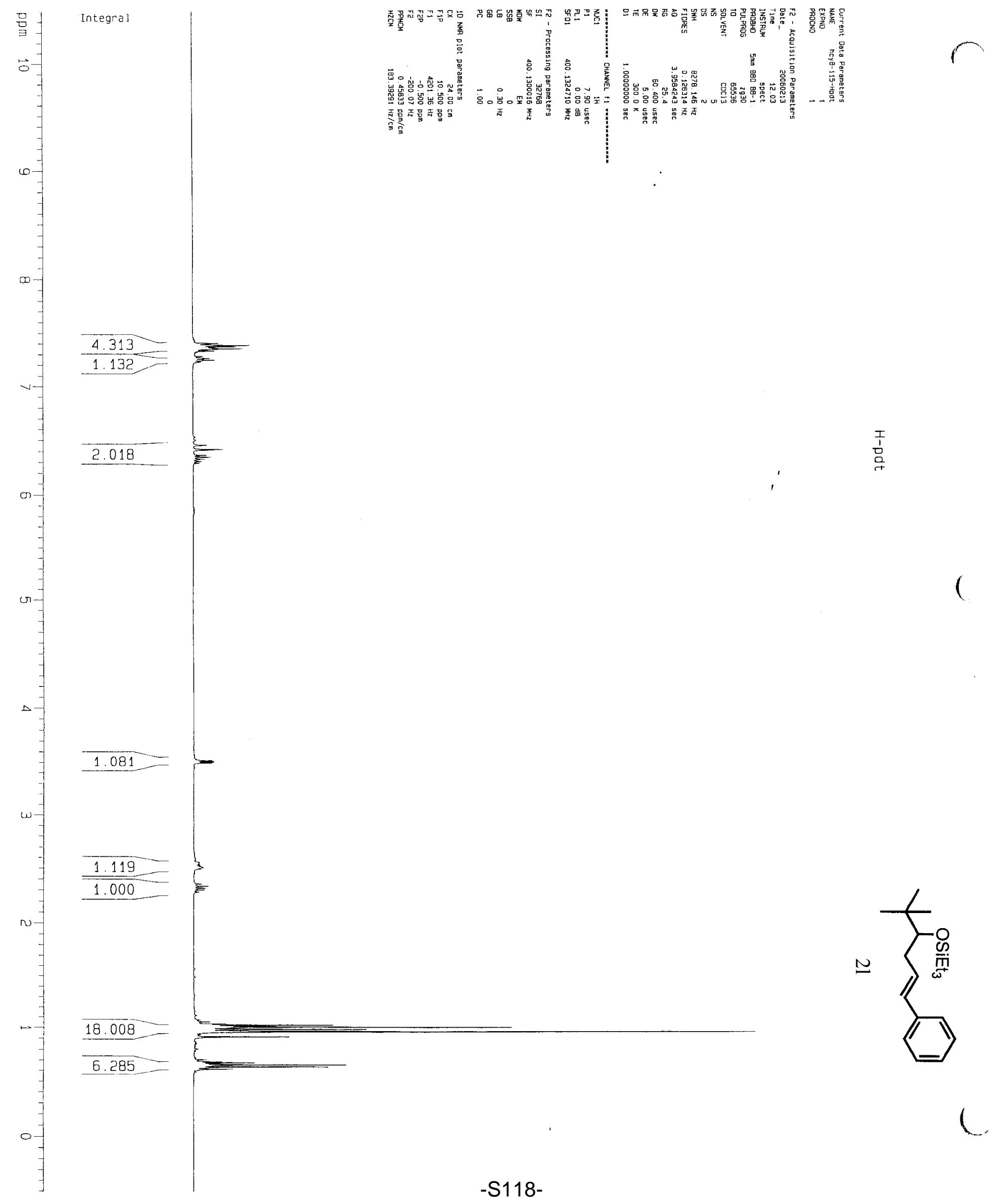




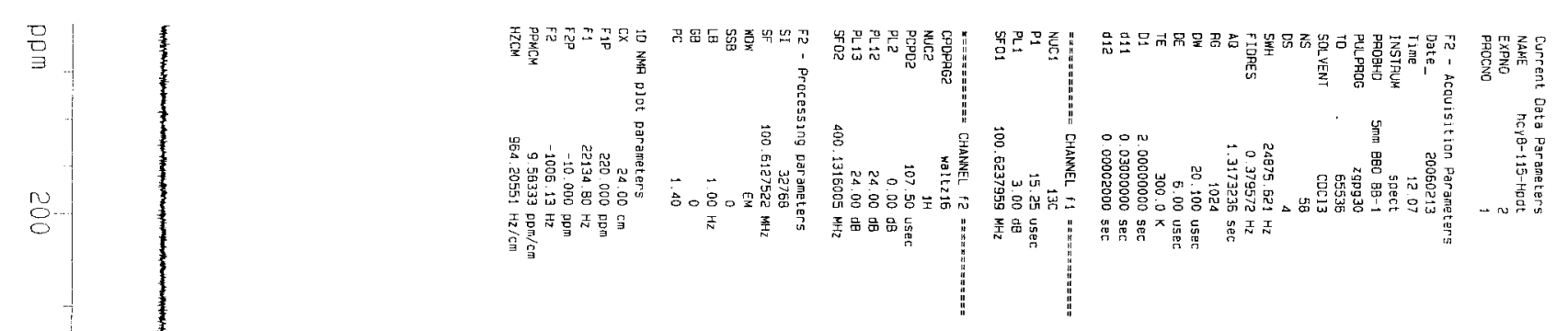

ppm

略

형

点

.
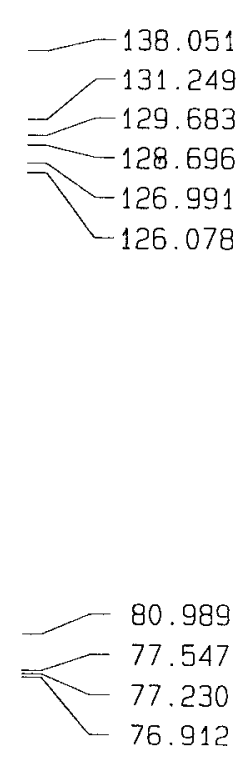

g

$\ddot{8}$

\section{8}

D 


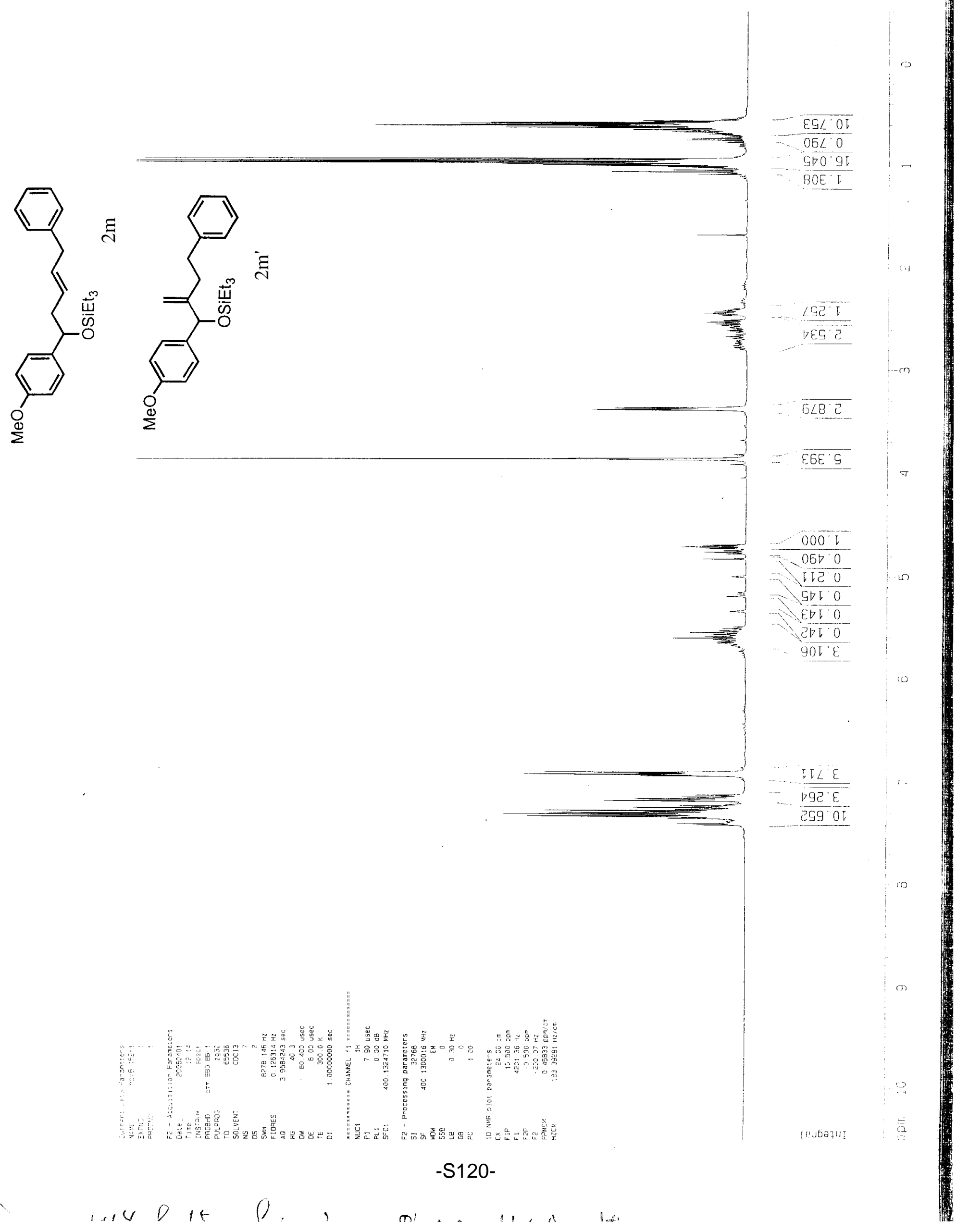




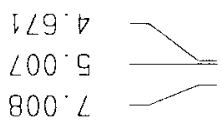

ฮี

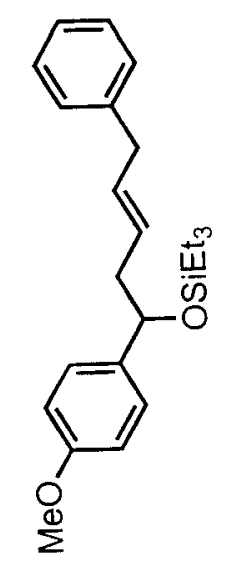

OSLEE

$890^{\circ} 6 \varepsilon$

Ogट $6 E+>$

$\angle E E^{\circ} \nabla D$

$\ldots$

$\nabla D E \subseteq \subseteq$

IES'

$258^{\circ} \nabla L$

हा6. $9 L$

IEZ $\angle L$

$805 \%$
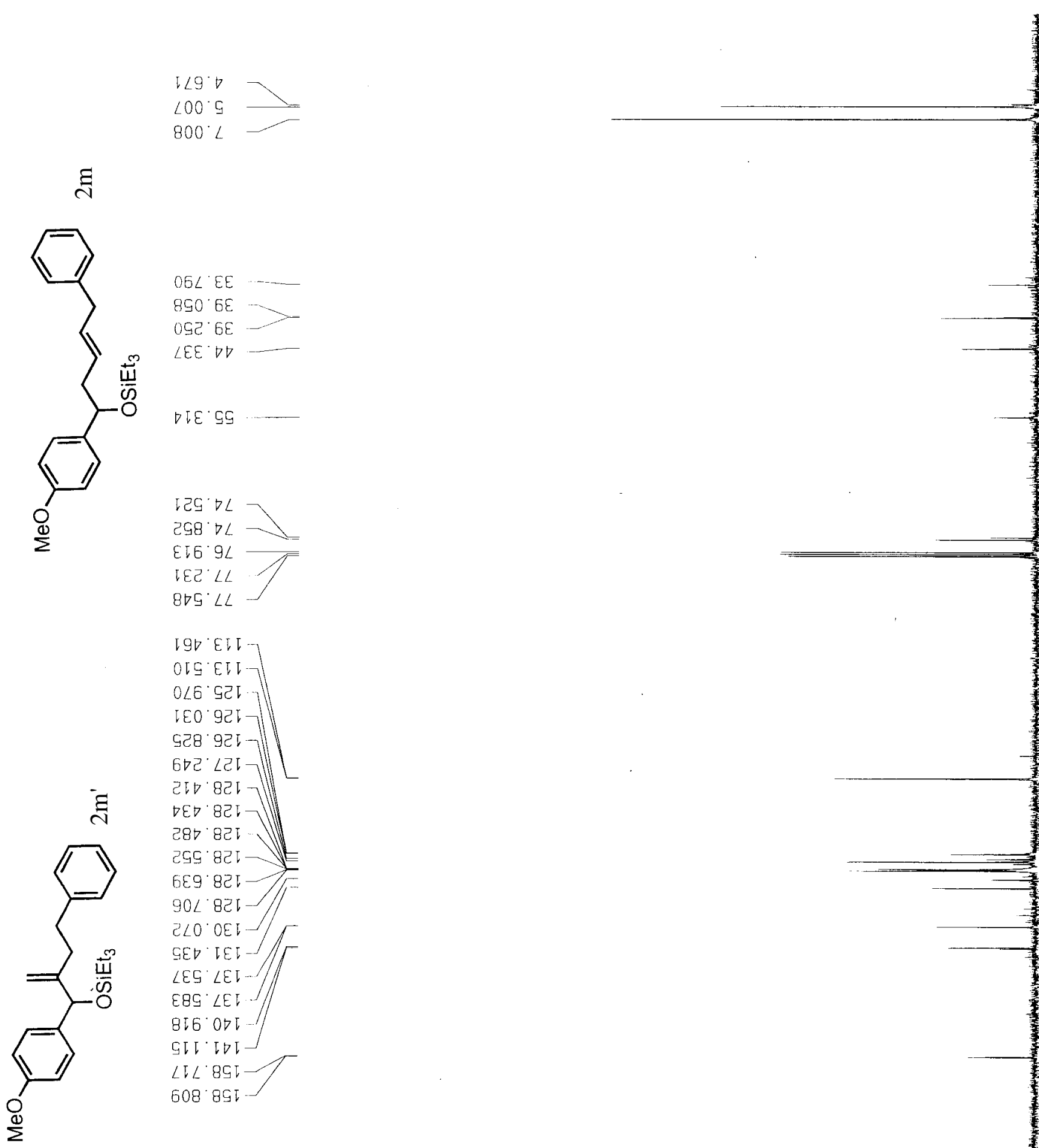

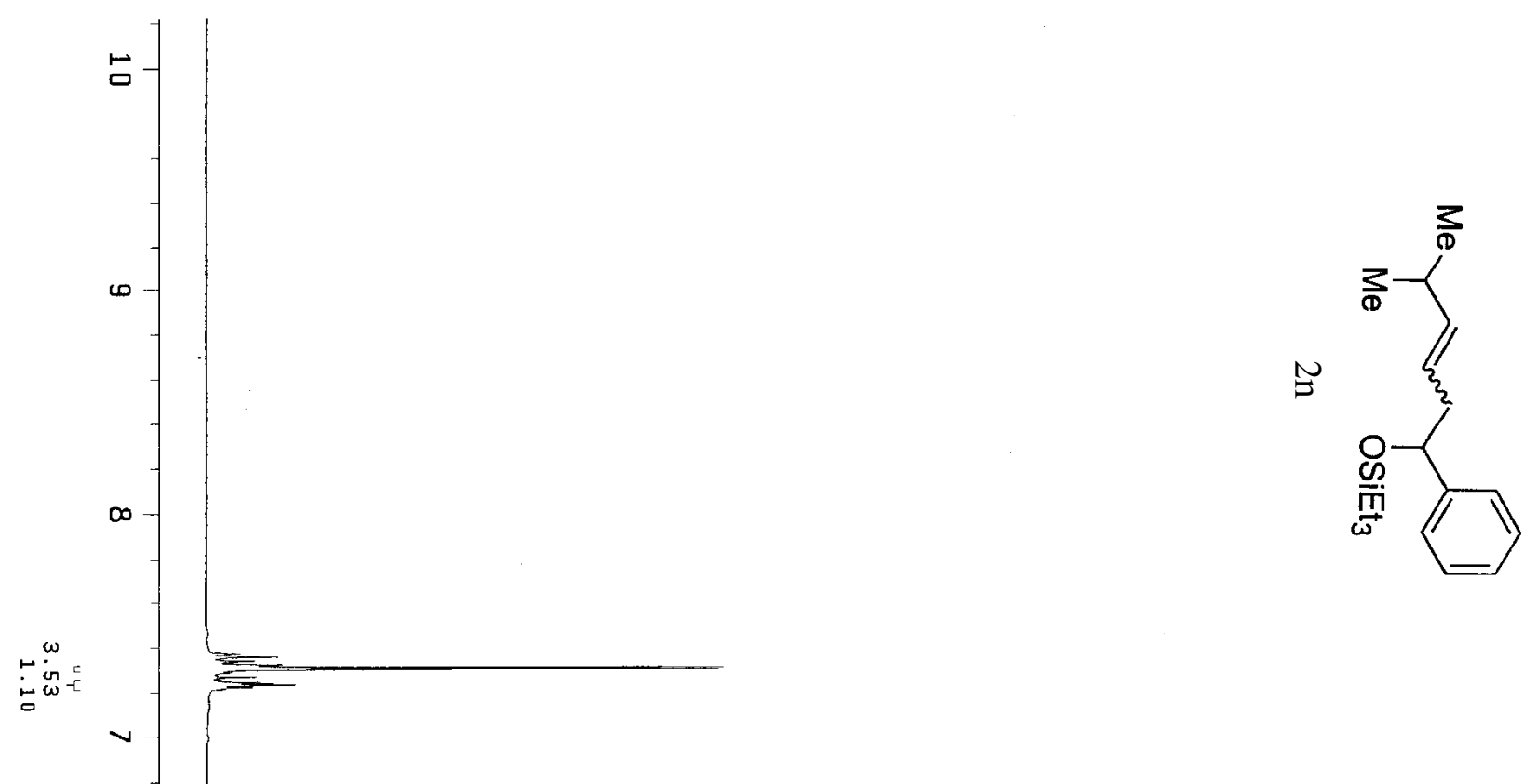

$\sigma$

in

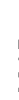




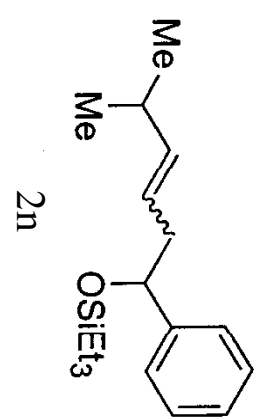




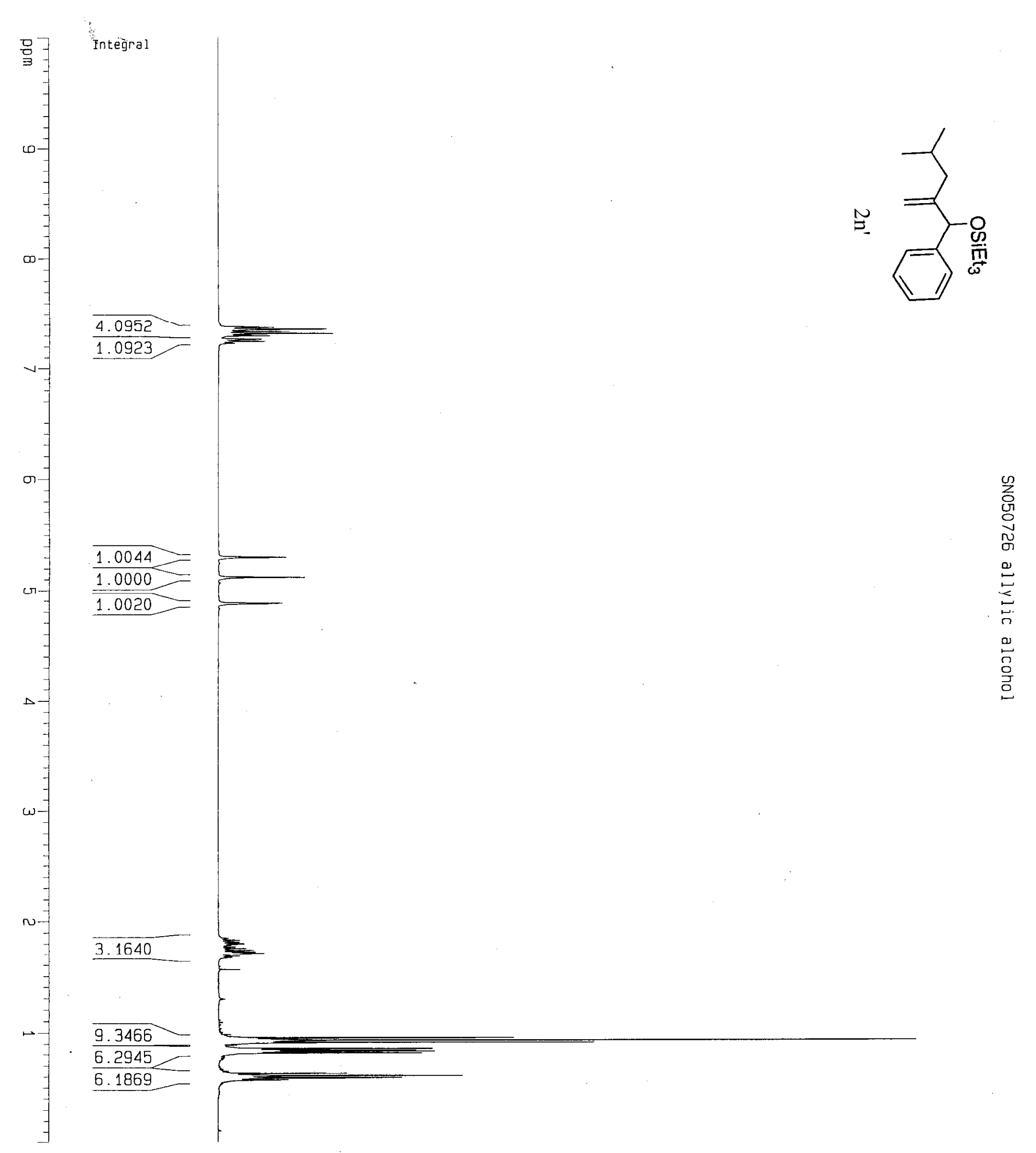

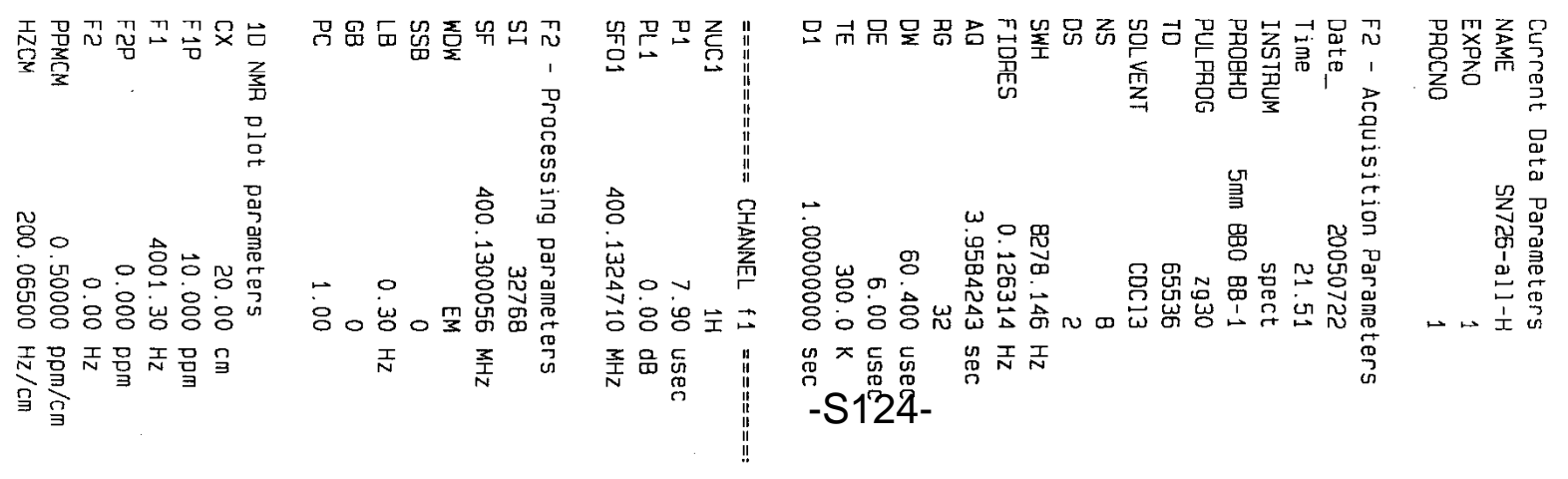




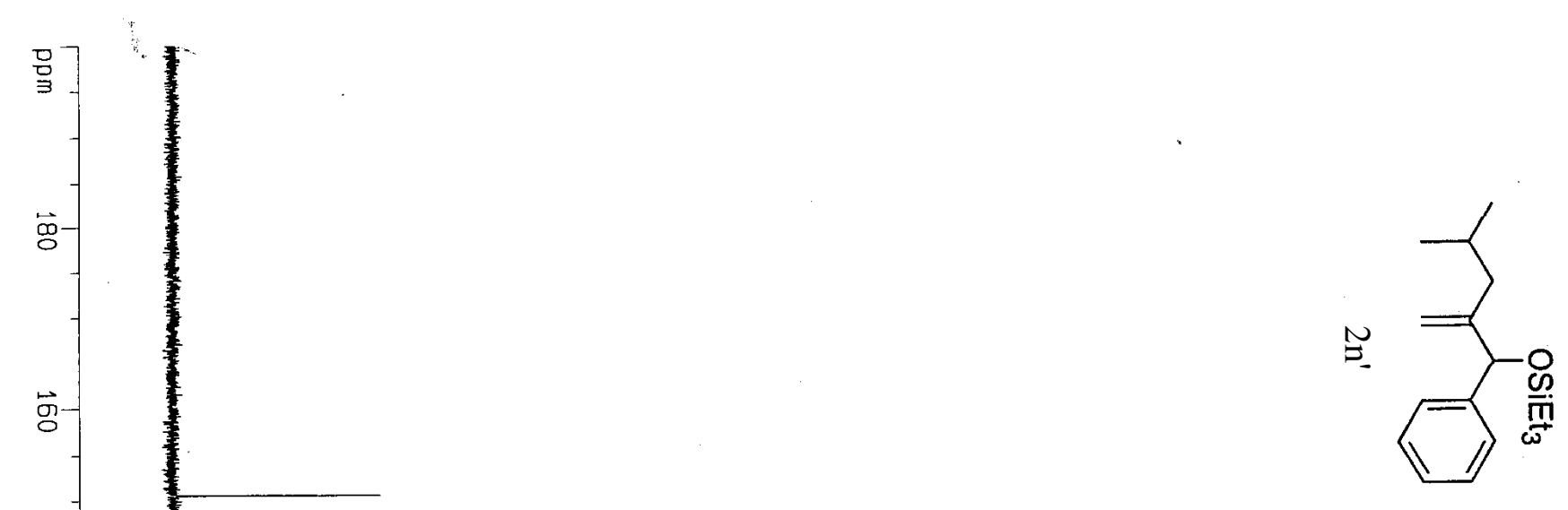

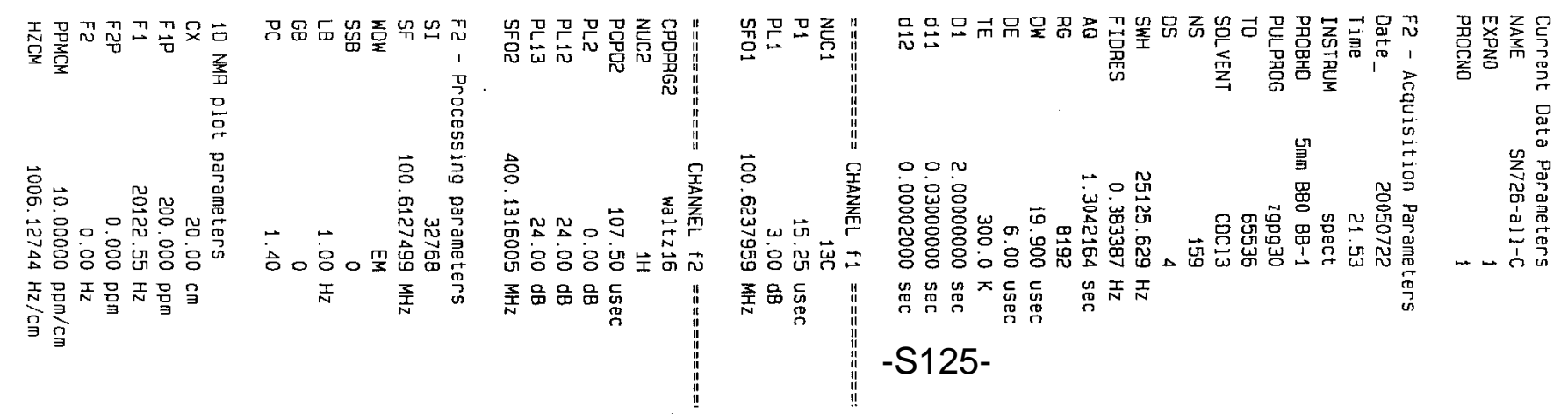




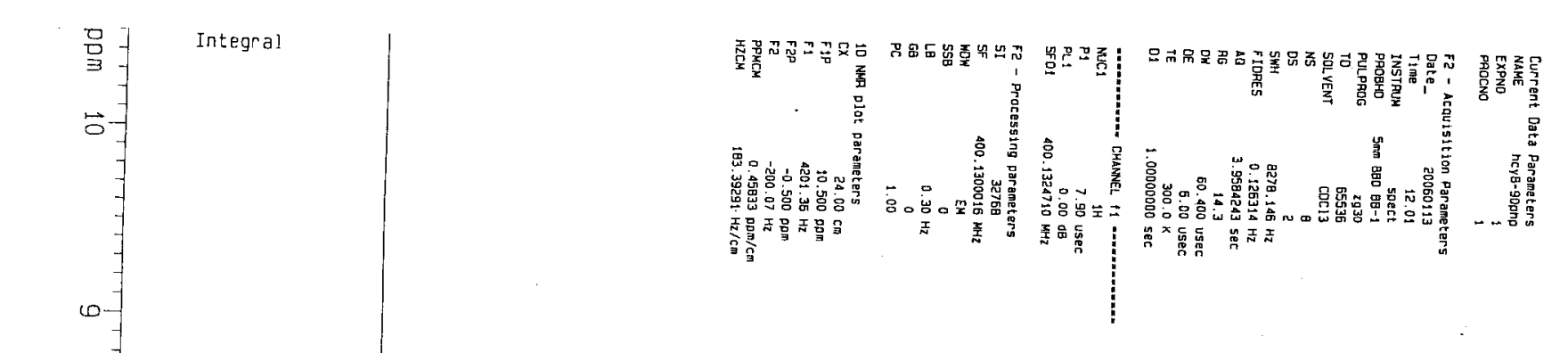

N
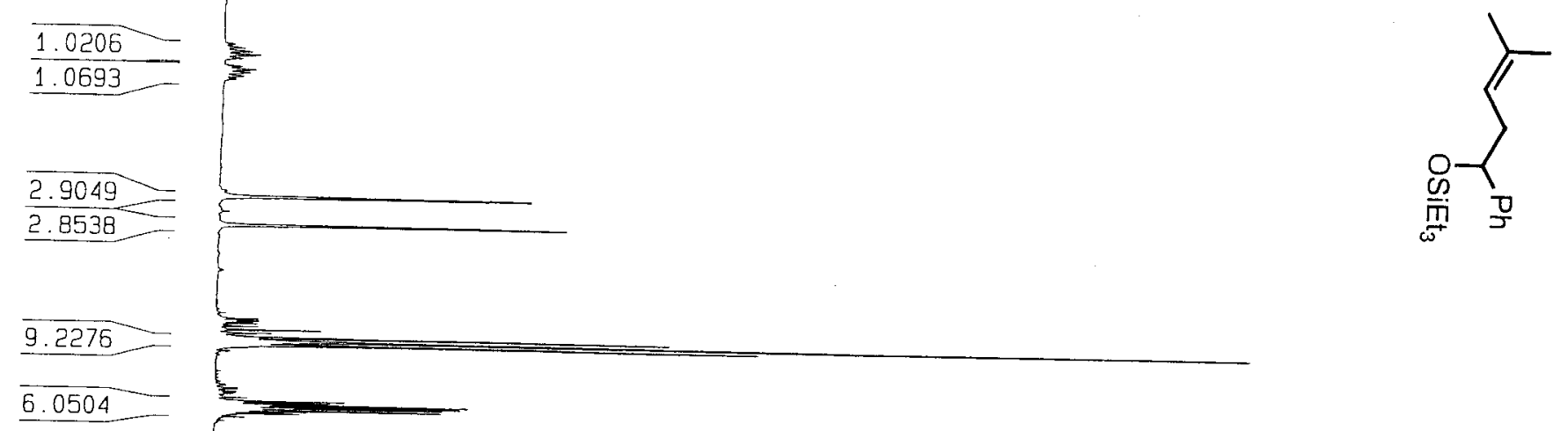


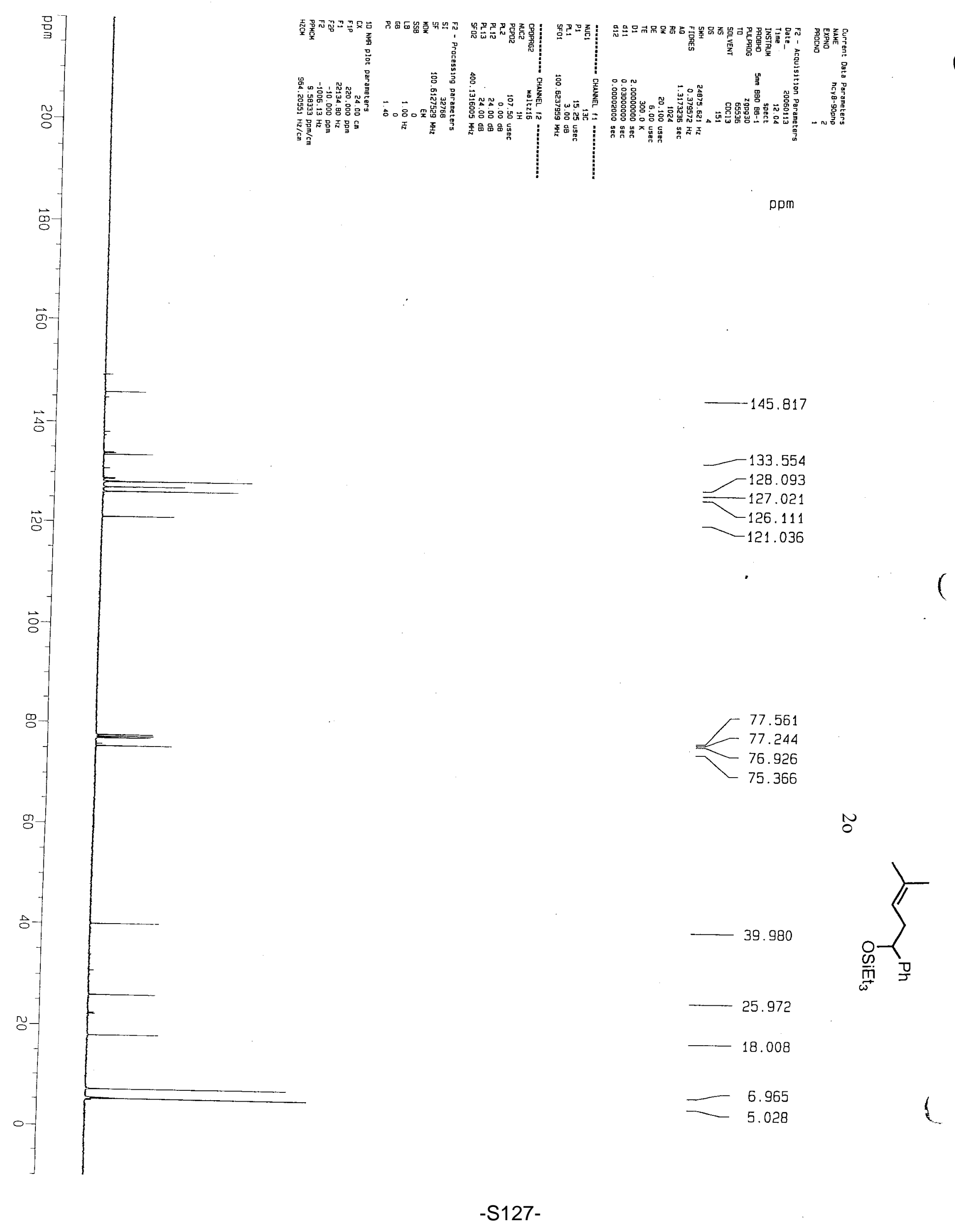




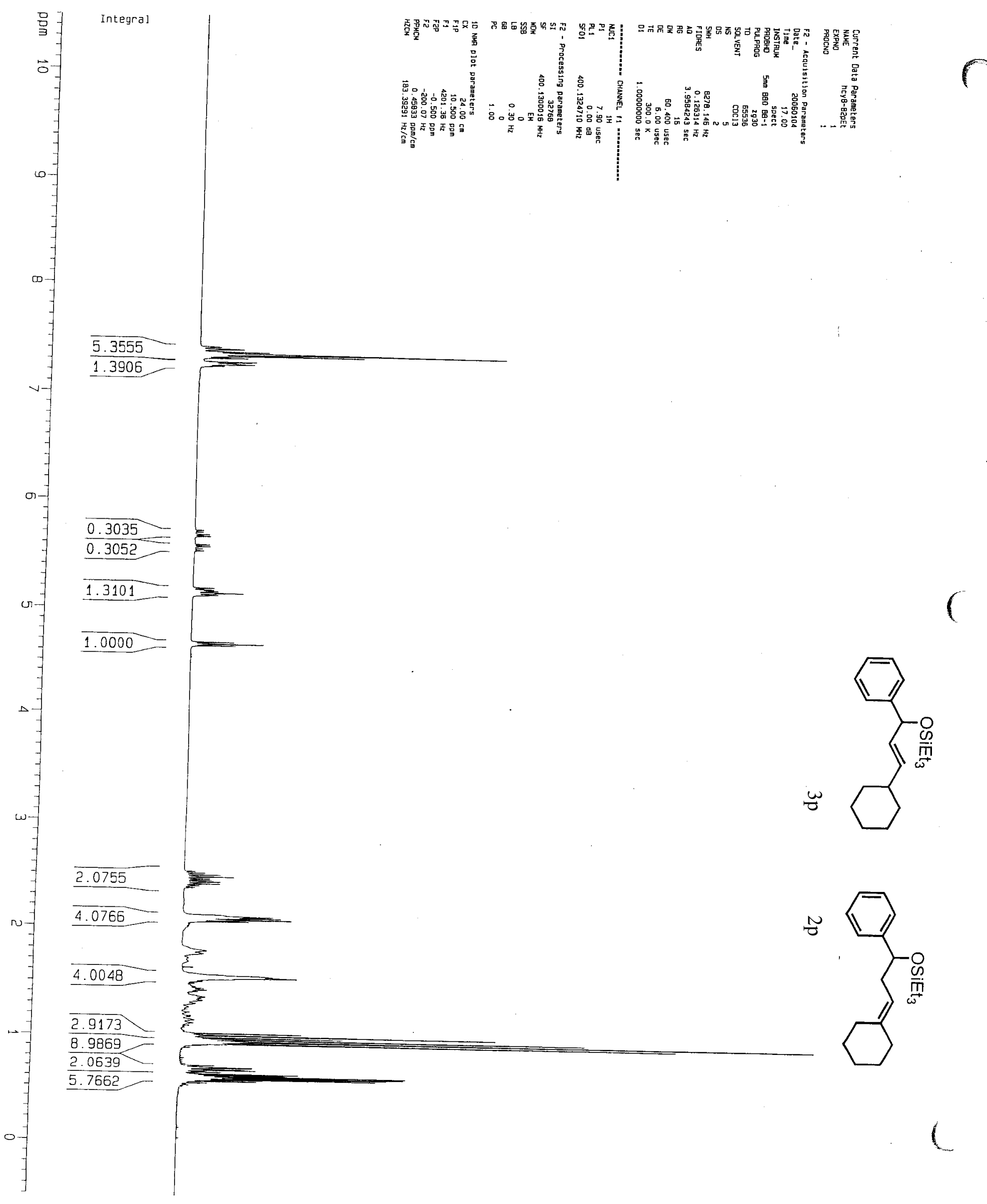




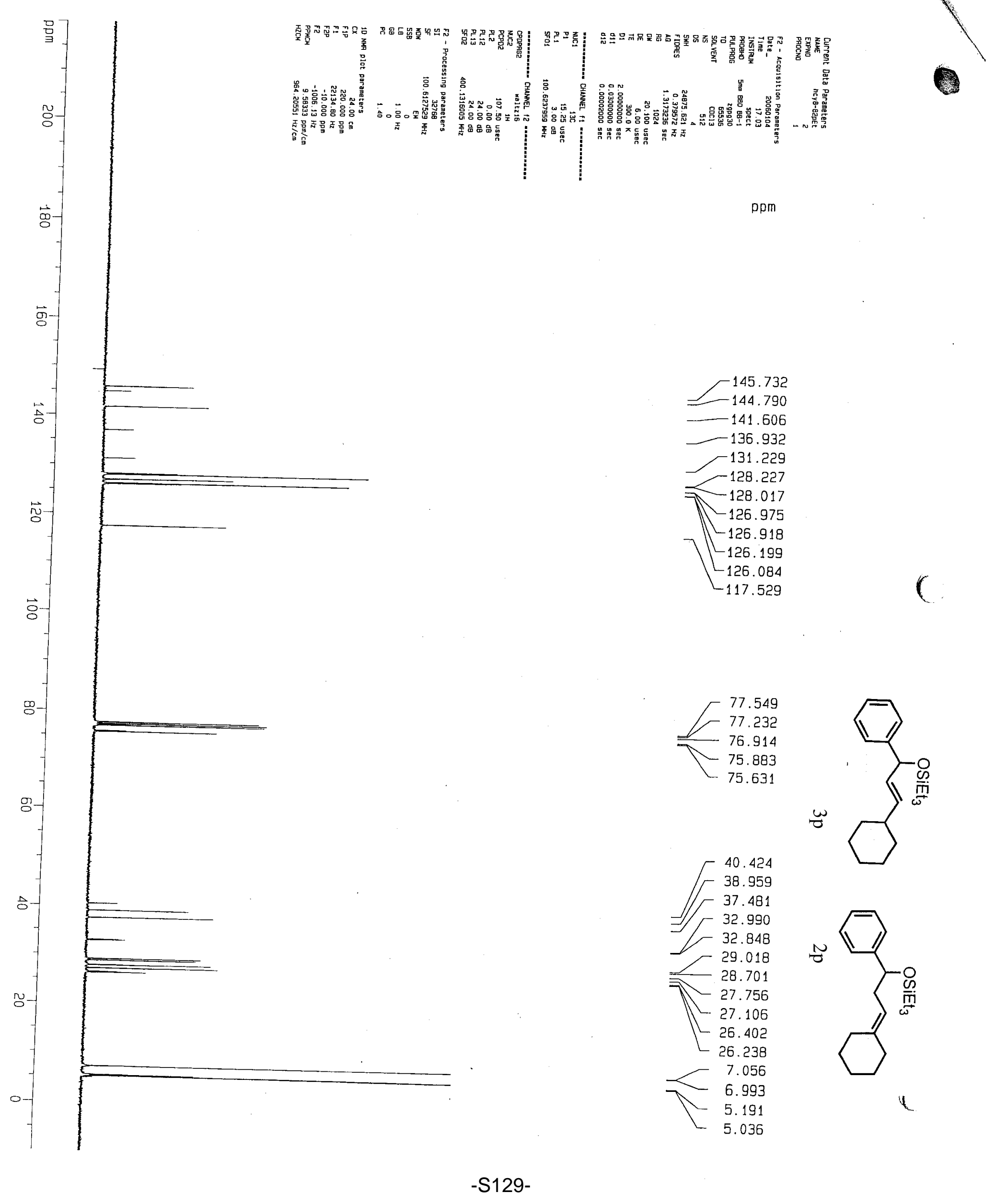



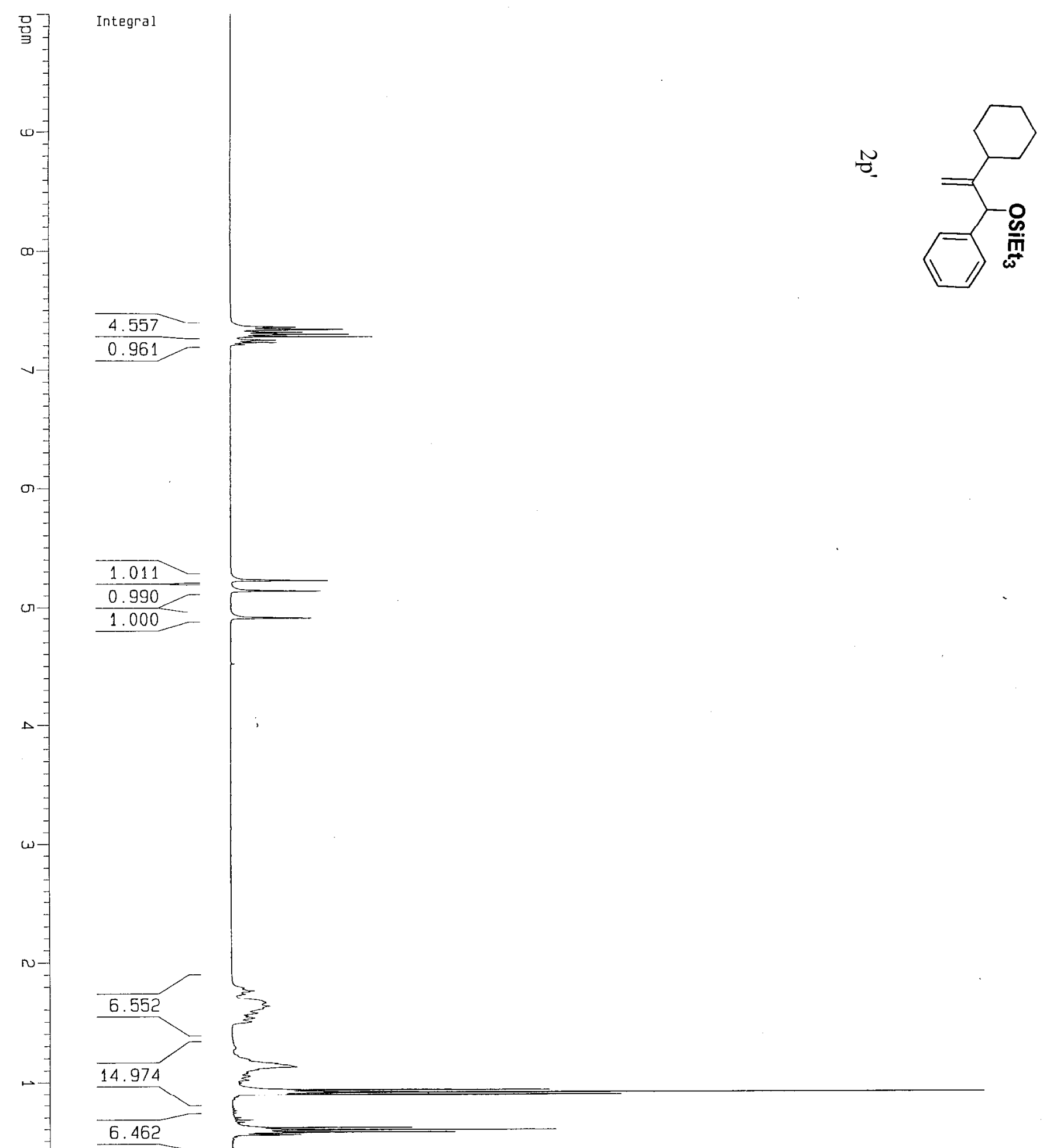

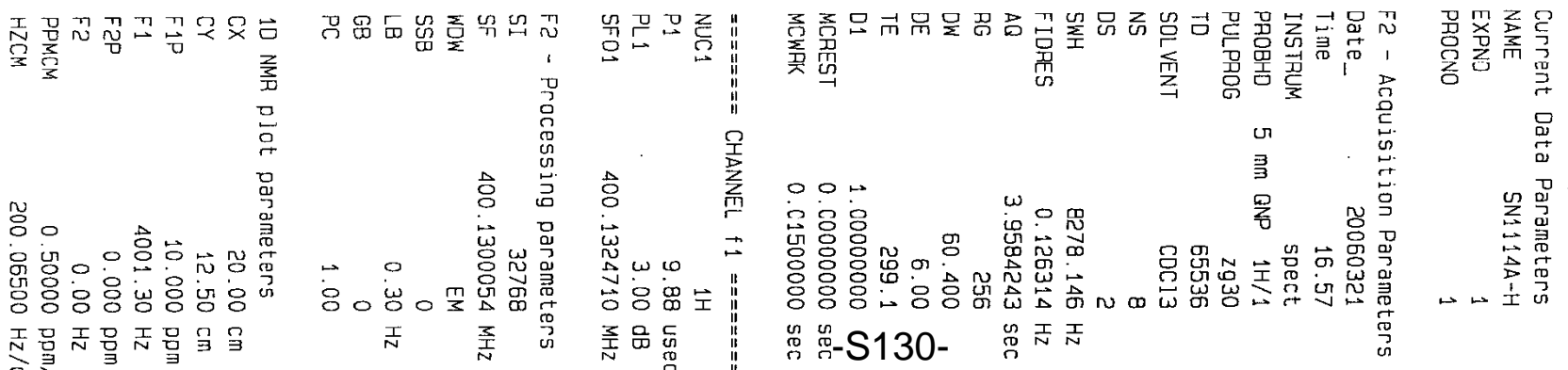
息

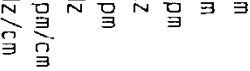



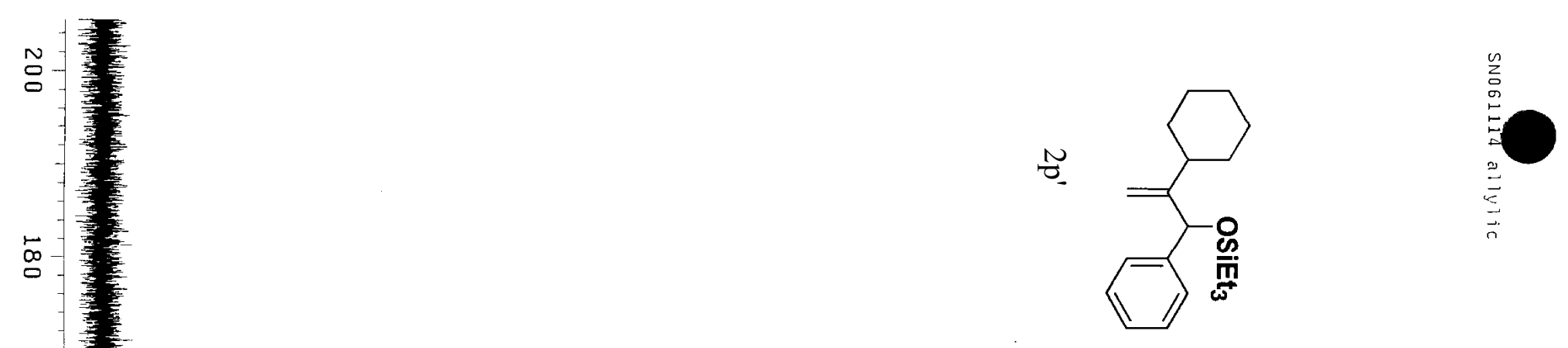

م

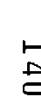

$\stackrel{n}{0}$

口-

-

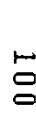

$\stackrel{\infty}{\circ}$

g

$E$

$+$

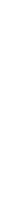




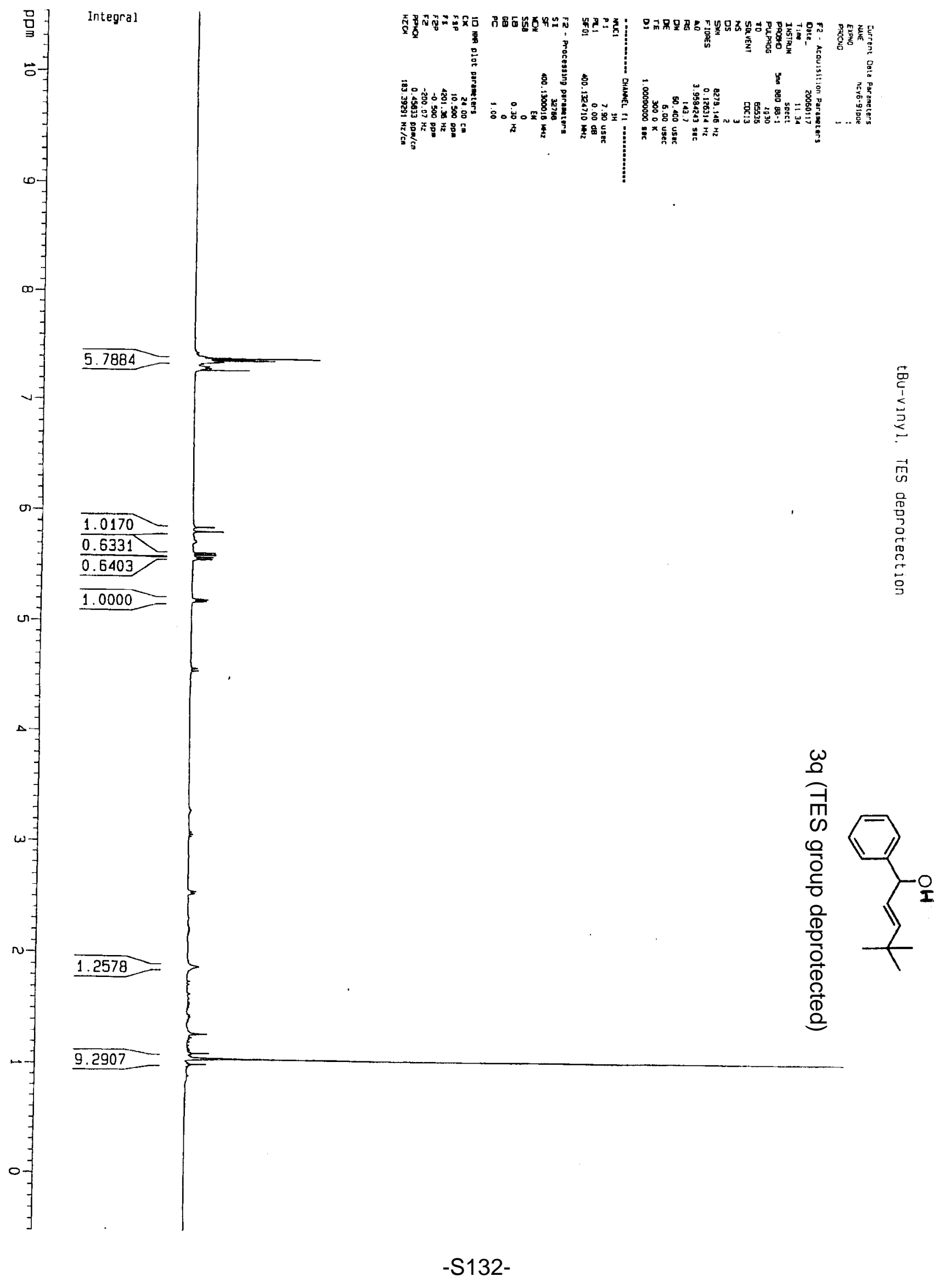




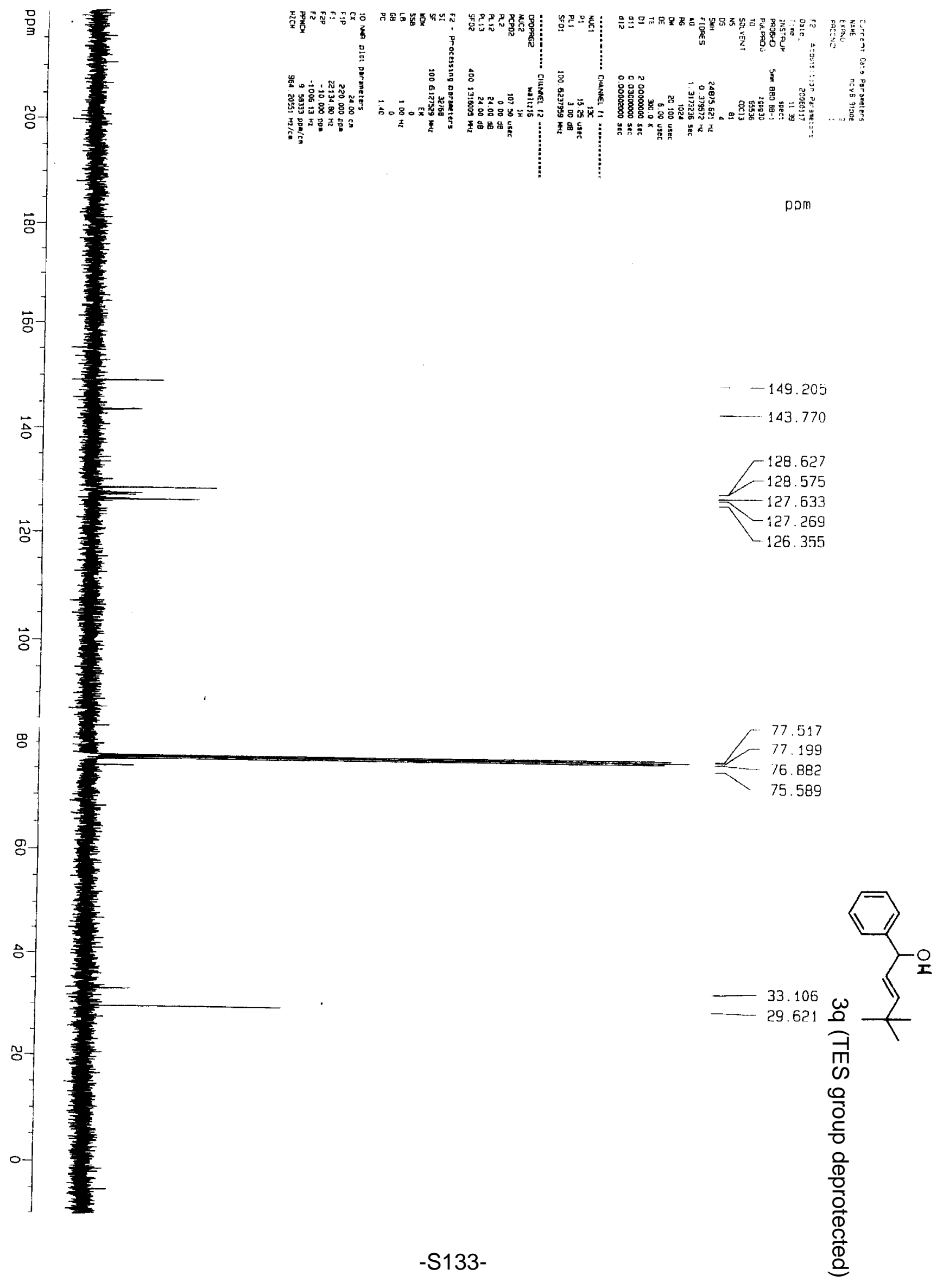



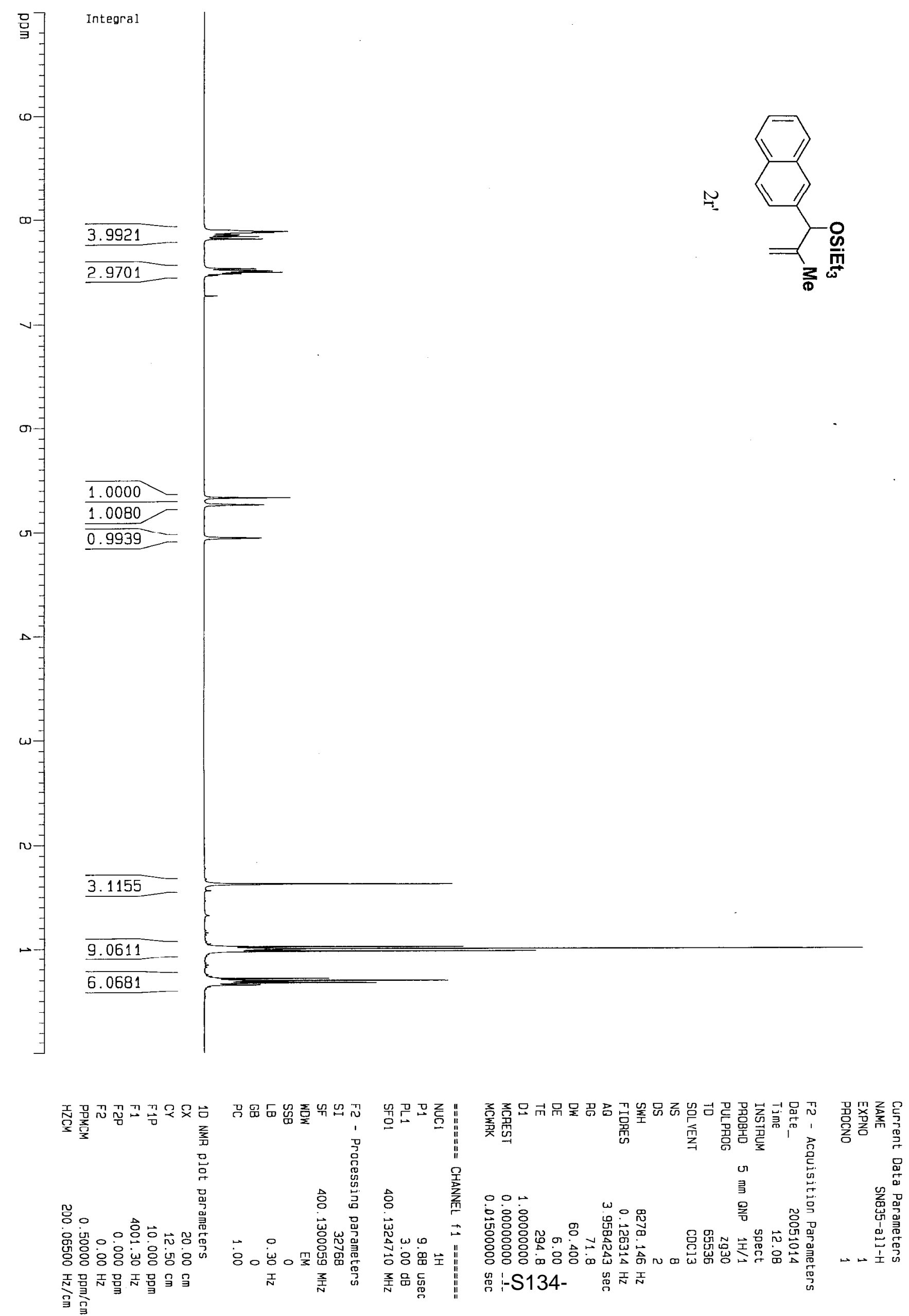

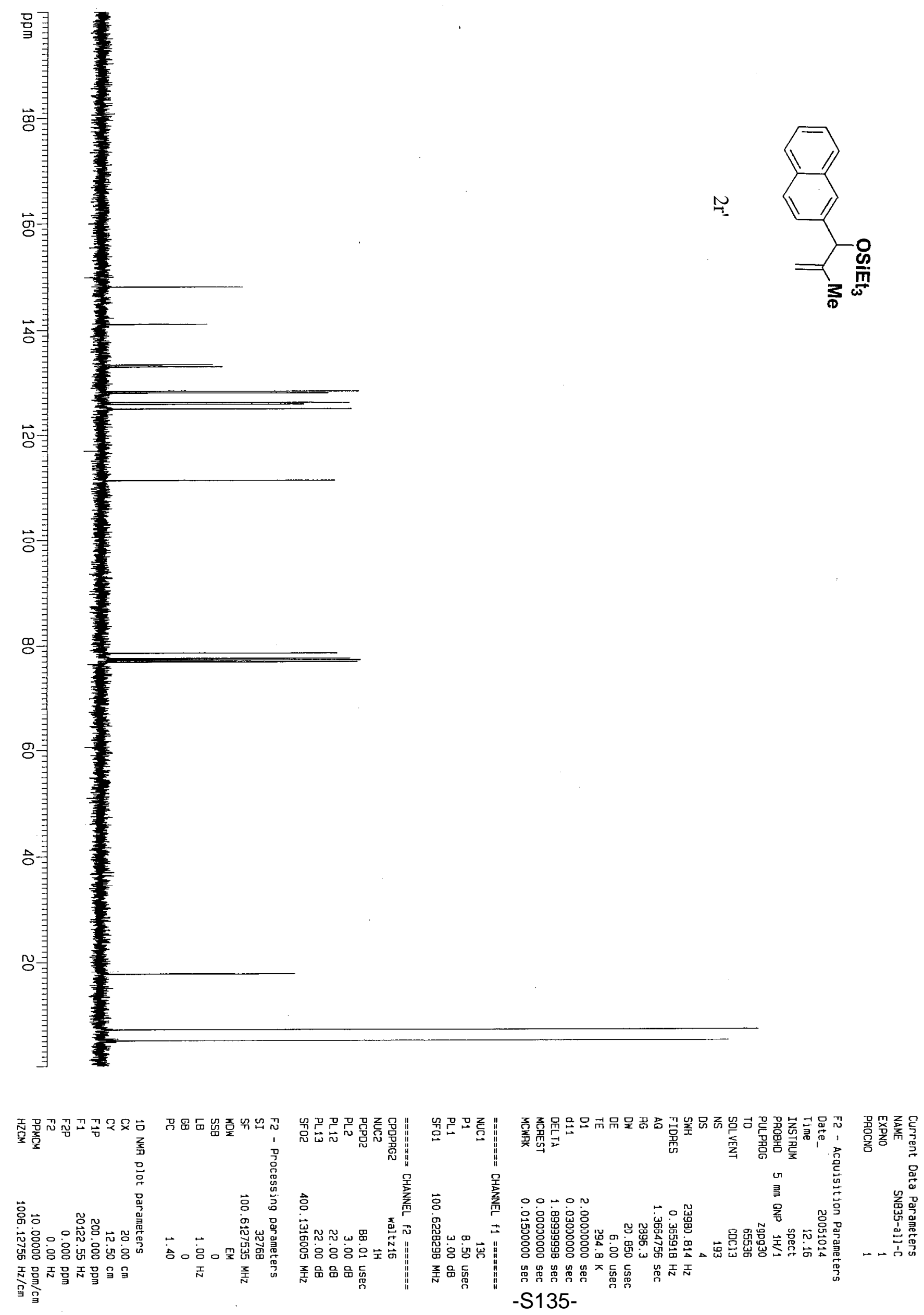

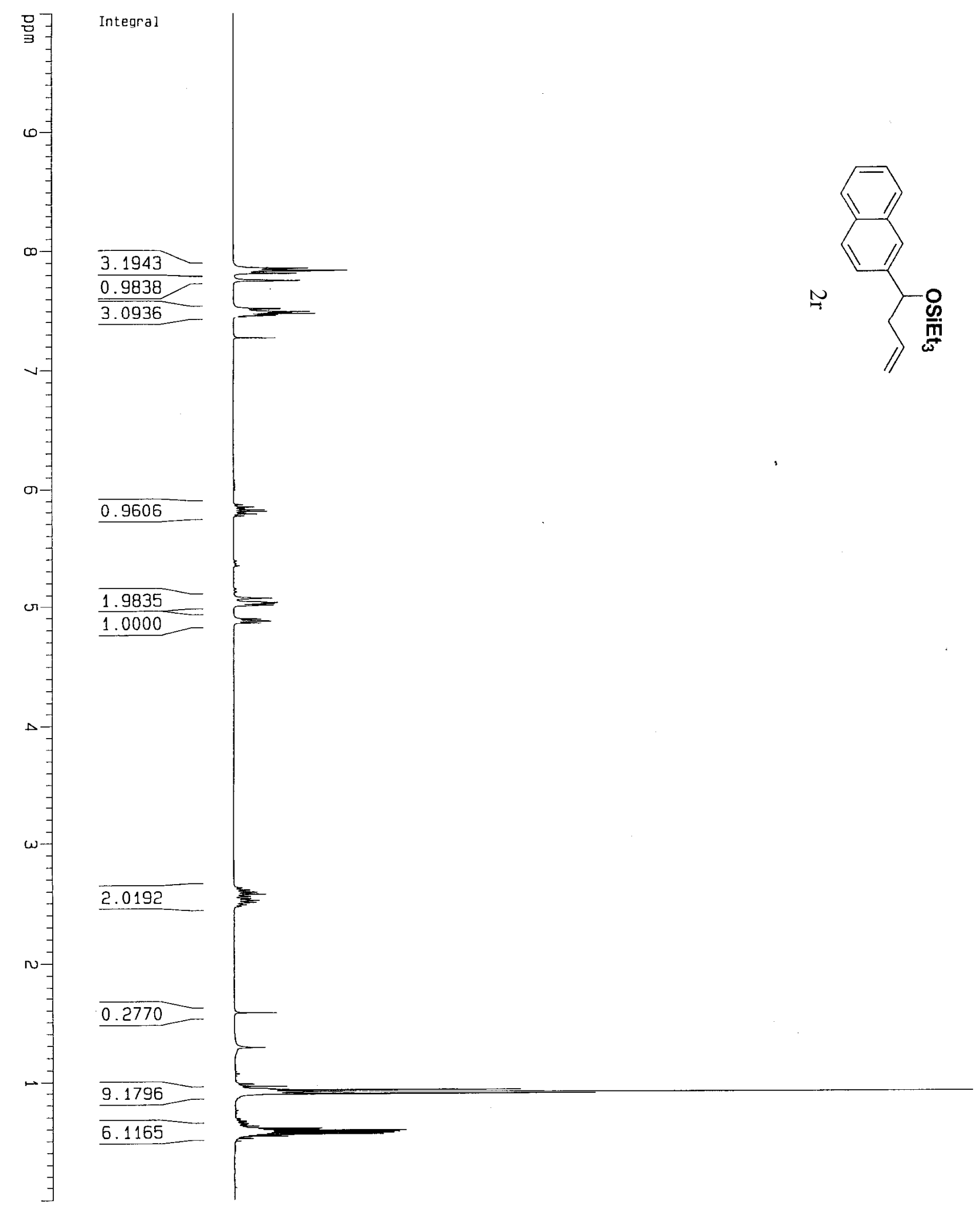

0
0
00
0
0
0
0
0
0
0
0
0
$\vdots$
$\vdots$
$\vdots$
0
0

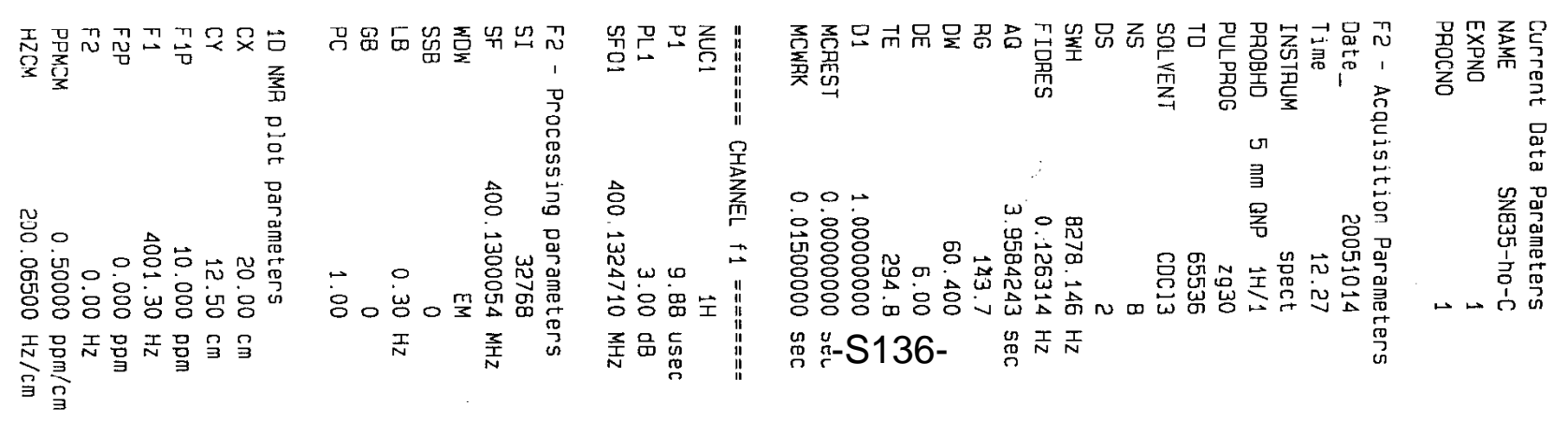




\section{ר}

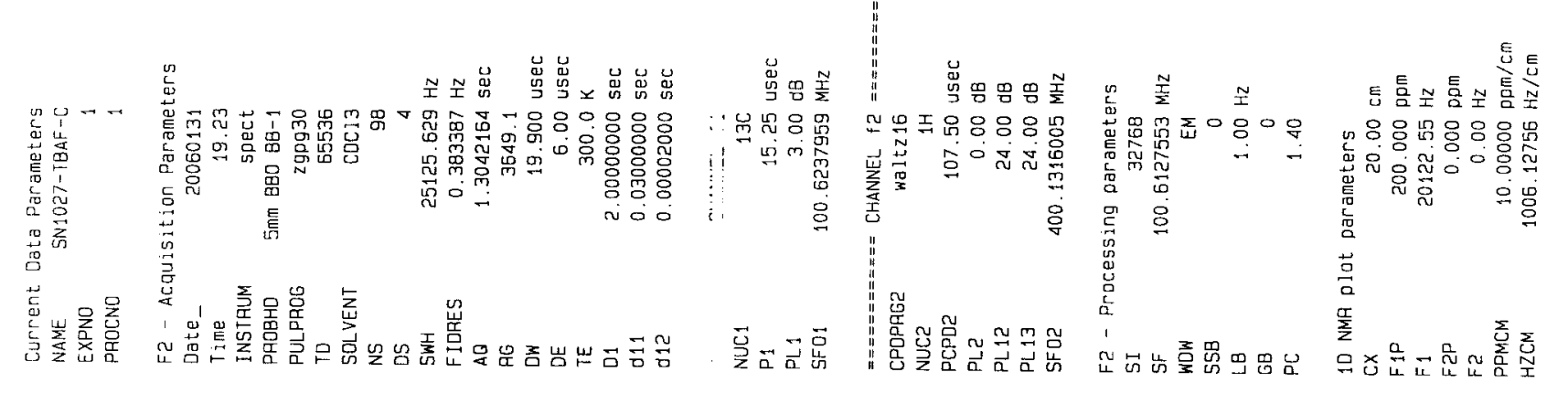

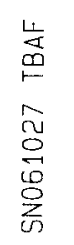
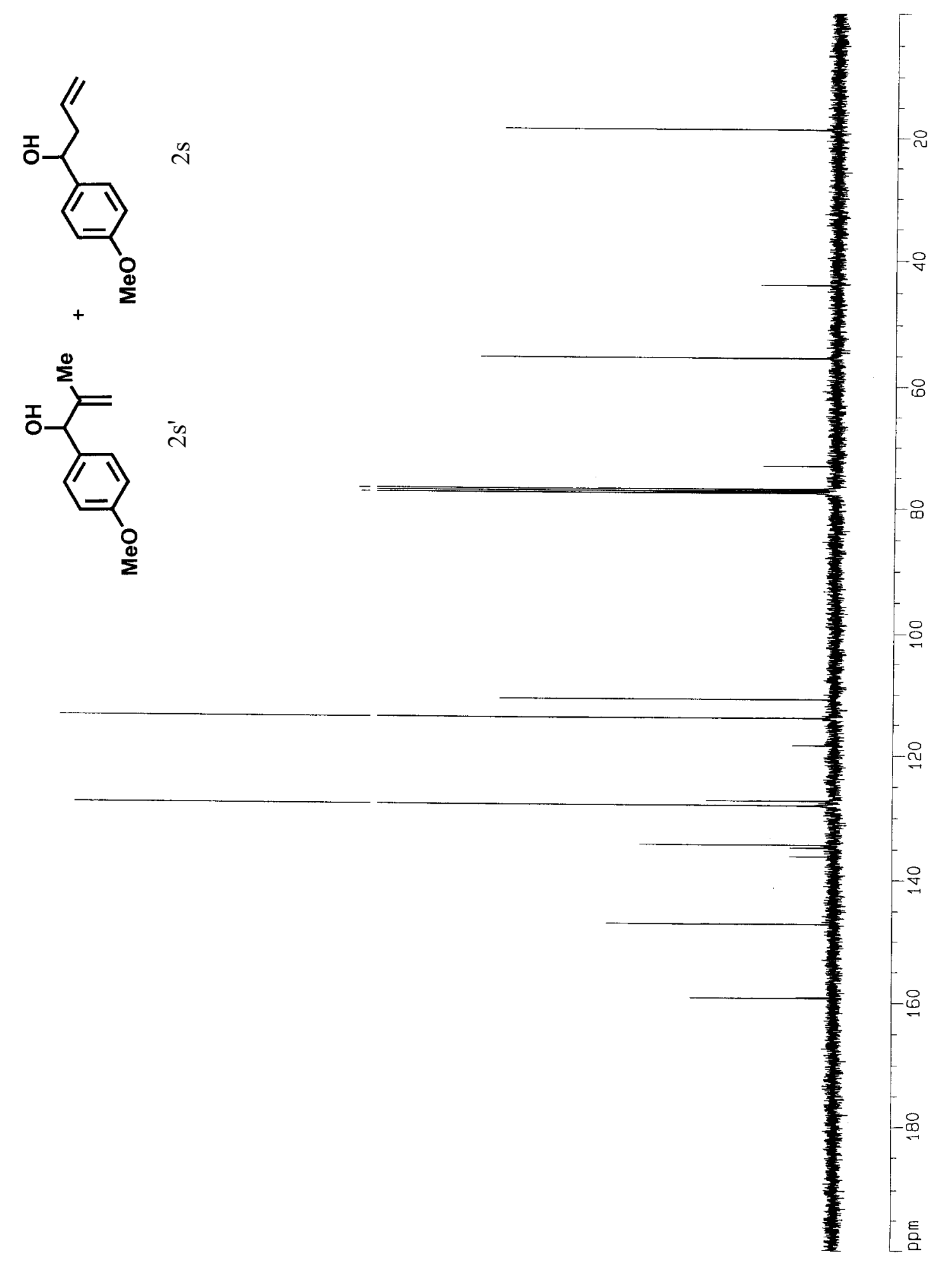


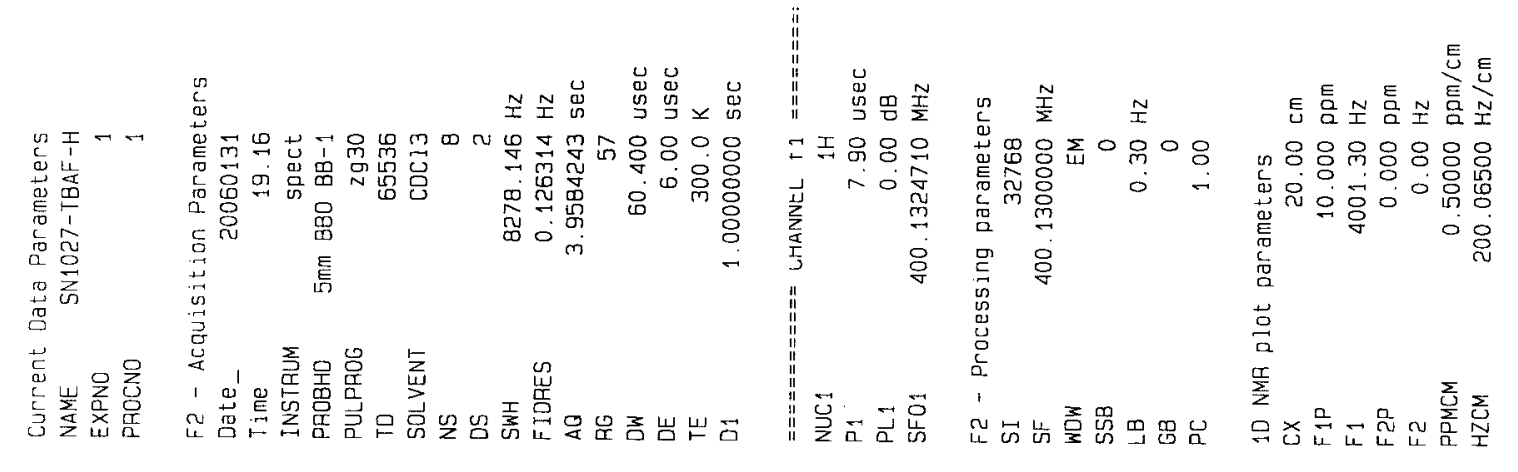

4
0
0
10
0
0
0
$ت$
0
0
0
0

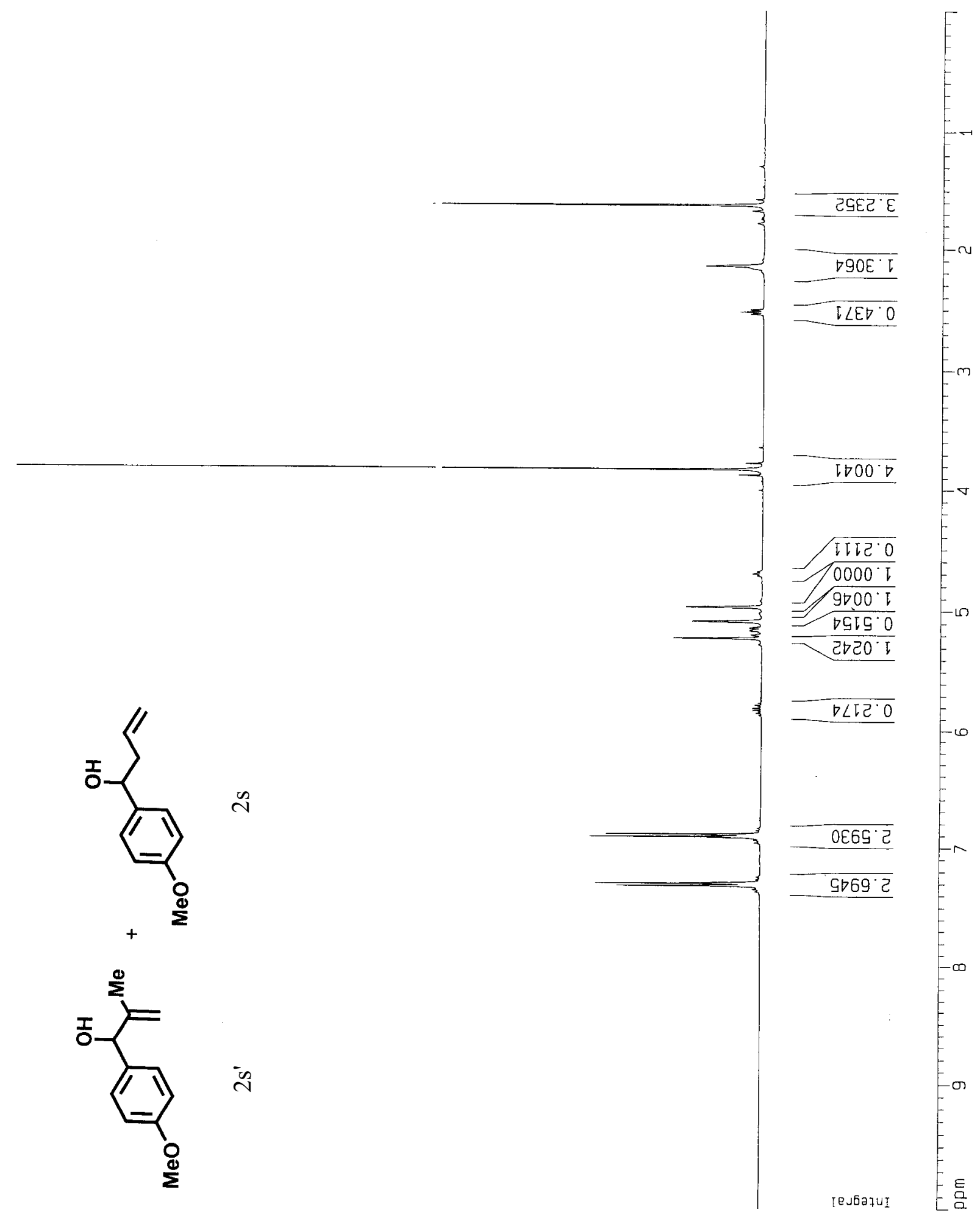






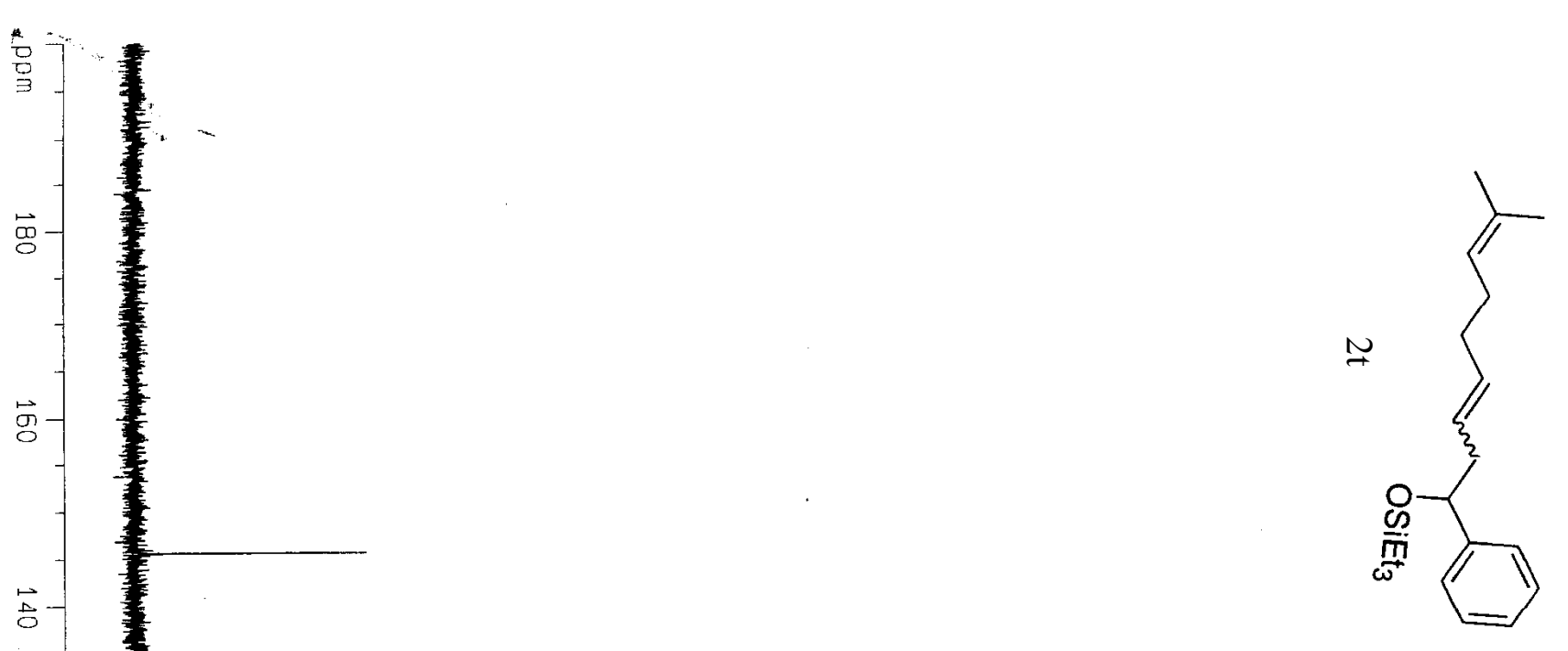




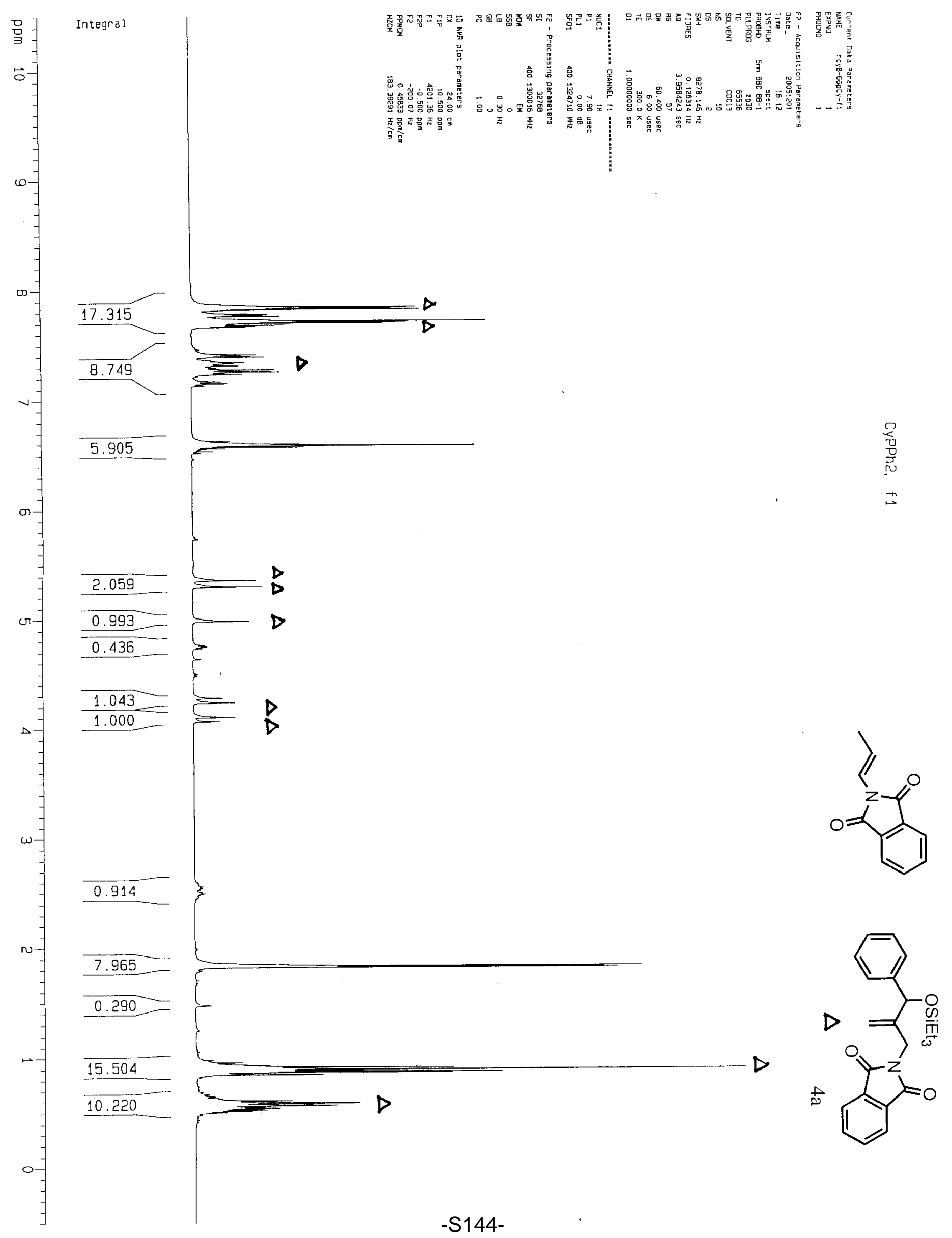



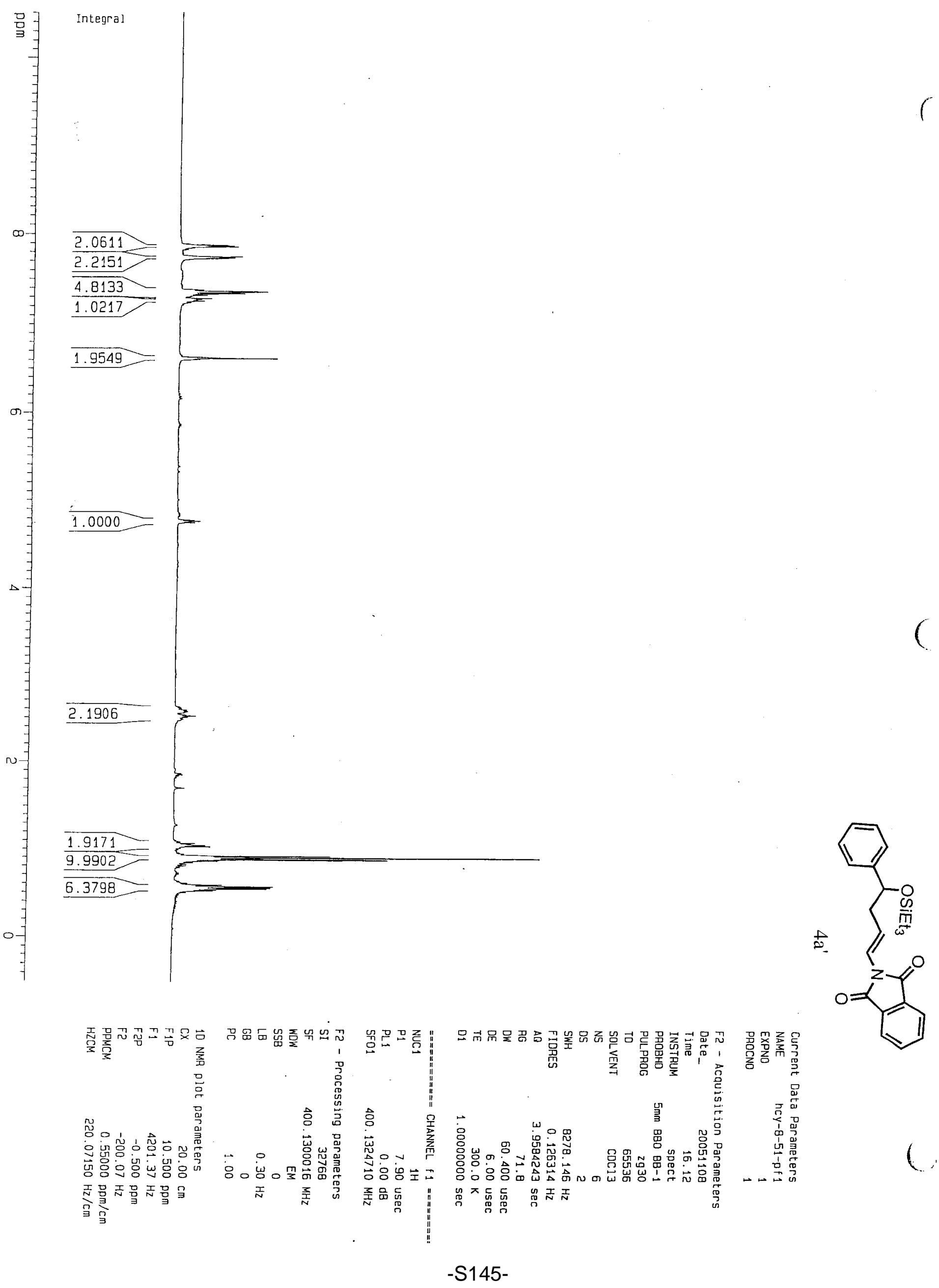


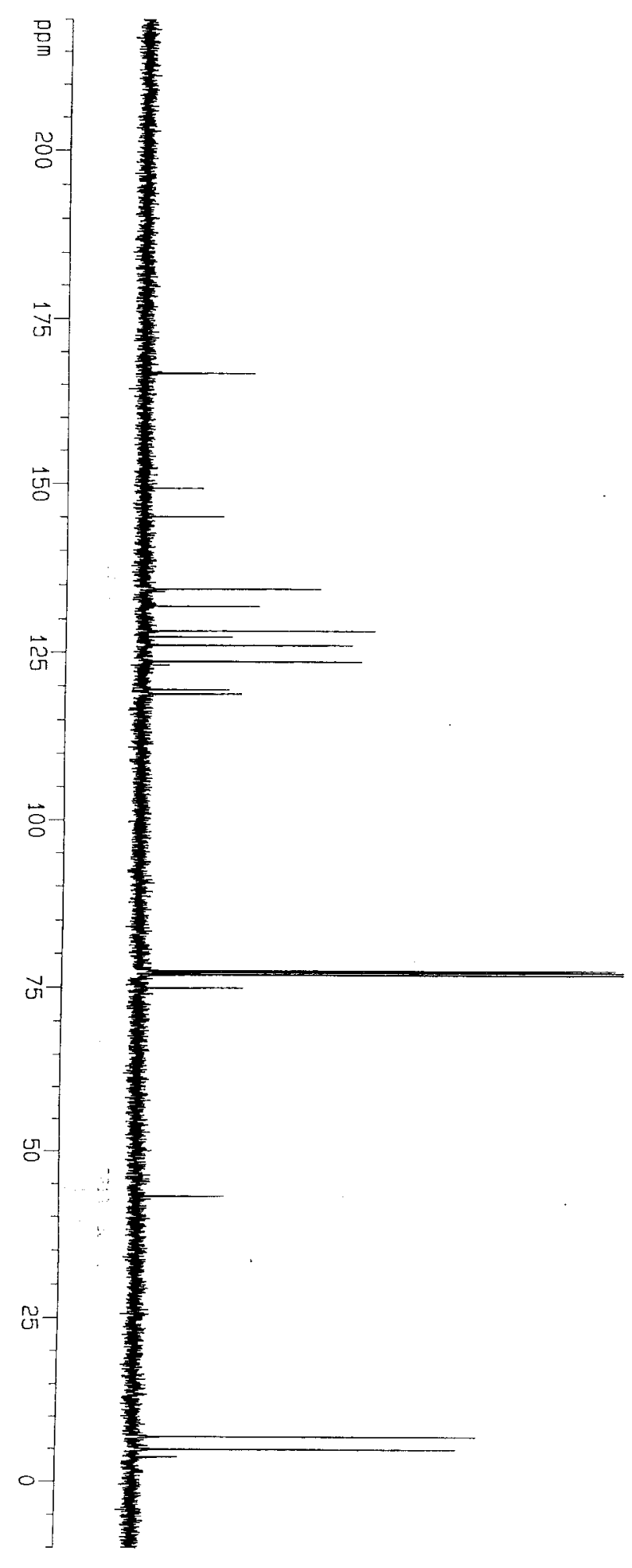

ก

$-166.687$
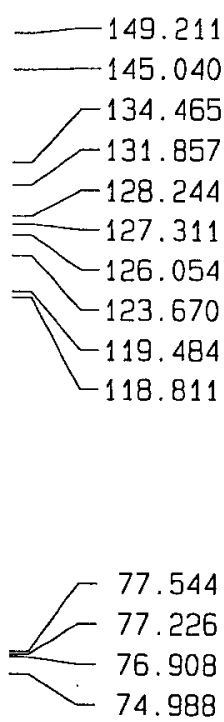

43.094
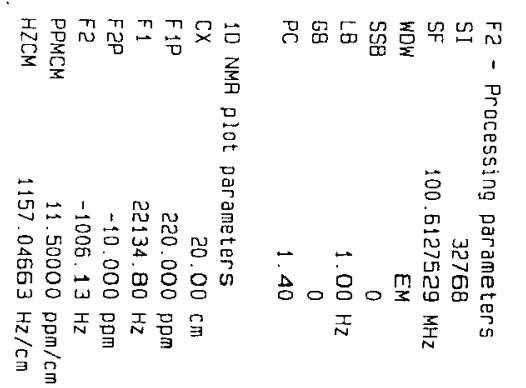

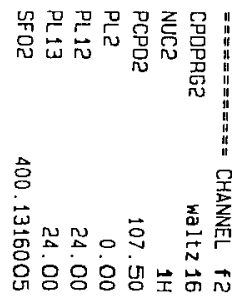

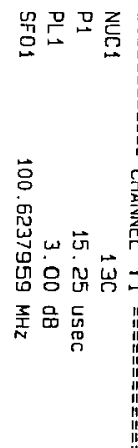

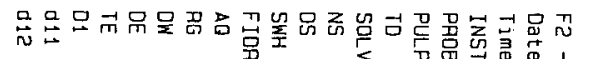

6. 961
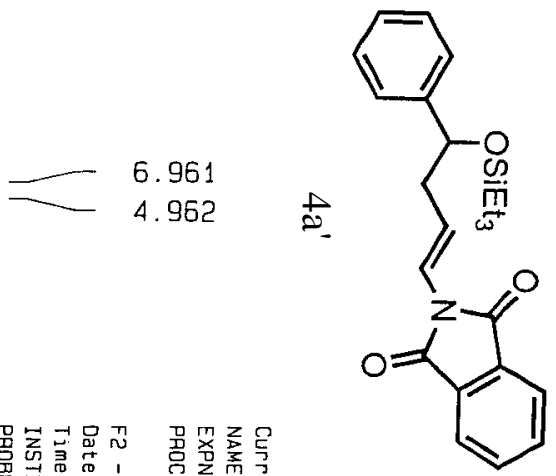

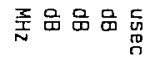

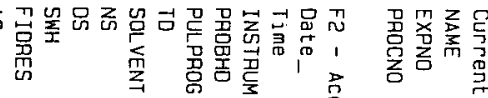

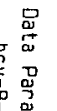



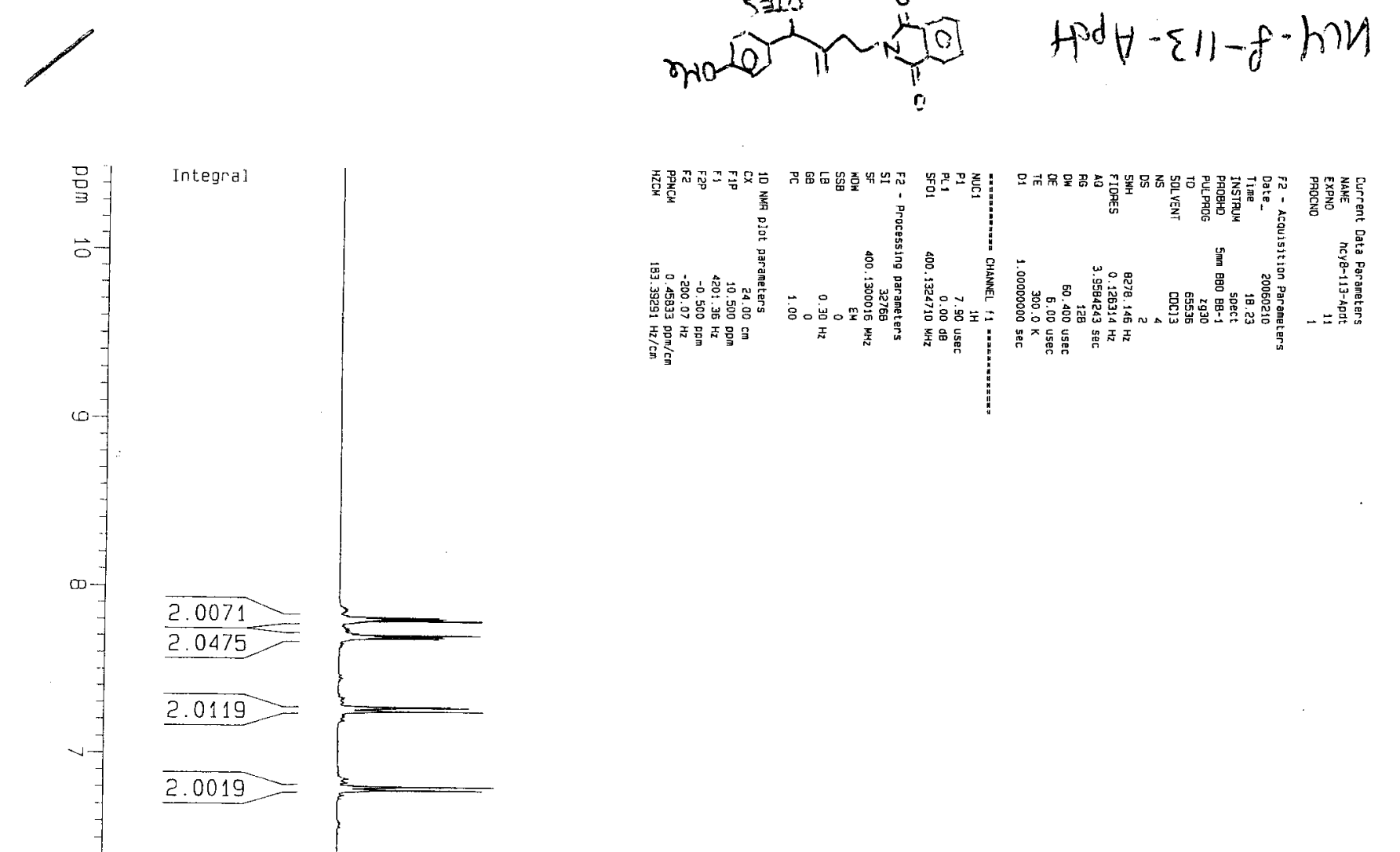

o

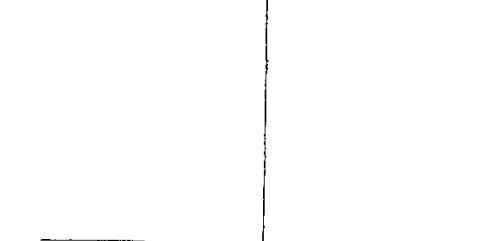

c
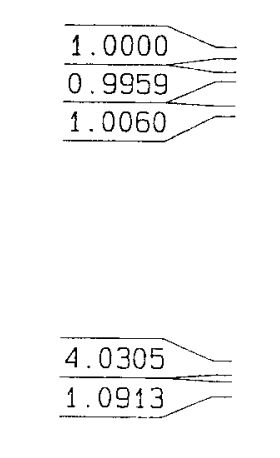

$\omega$

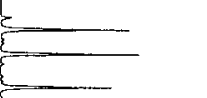

$D$
$\frac{1}{0}$
$\frac{0}{r}$
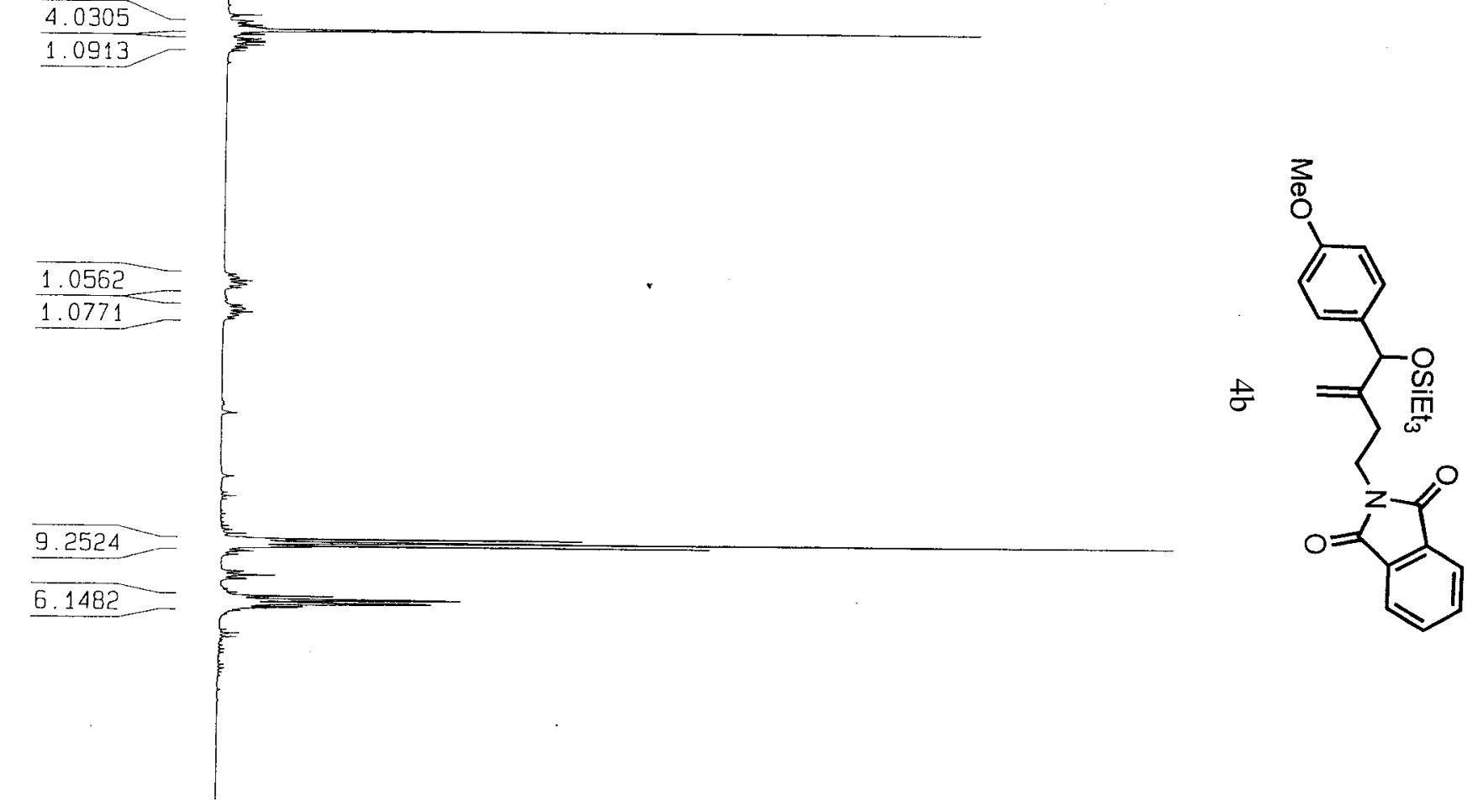


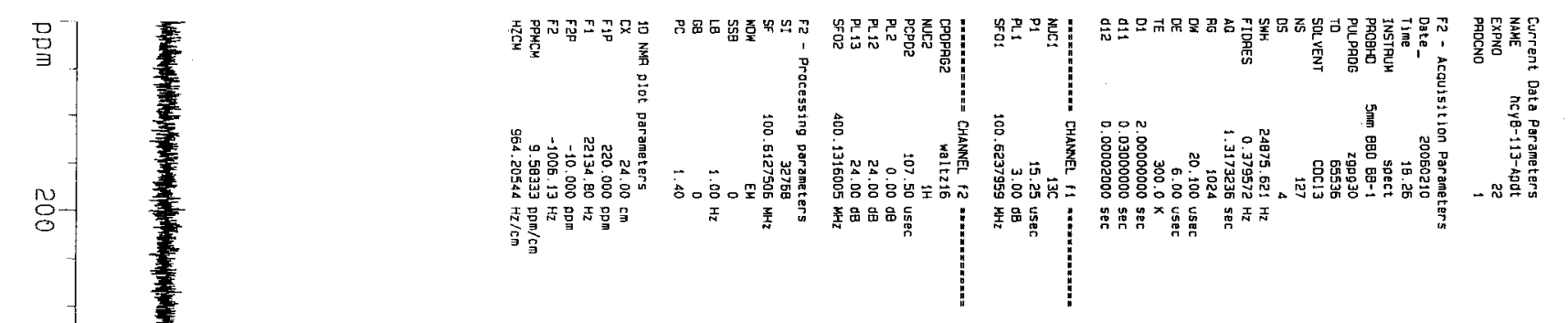

ppm

$\stackrel{\mathrm{O}}{\mathrm{O}}$

宊

$-168.348$

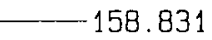

$-148.546$

$\overrightarrow{\mathrm{B}}$

.

$\begin{array}{r}135.171 \\ -\quad 133.947 \\ \hline\end{array}$

$-132.310$

맘

$-127.701$

123.255

ก.
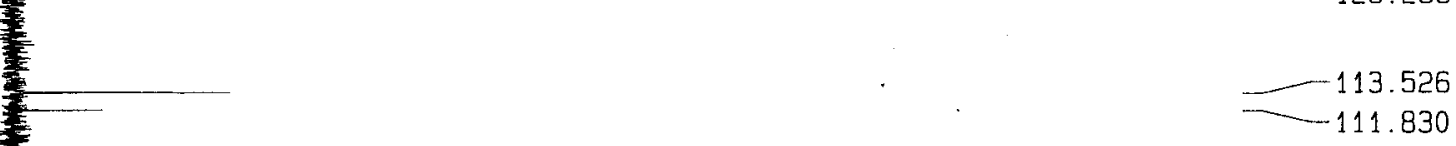

$\ddot{8}-$

g
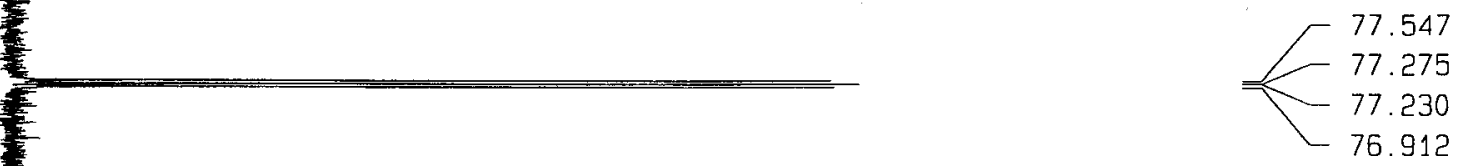

g-
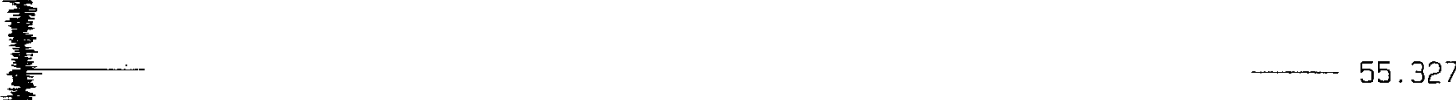

잉

के

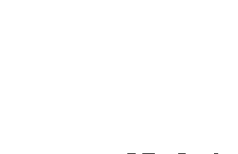

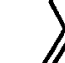

37.240

29.752

$-\quad 7.029$
$-\quad 4.967$

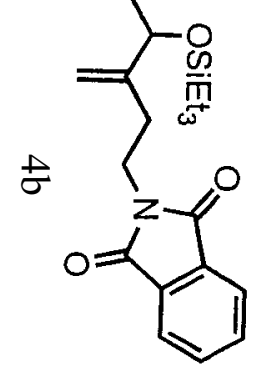




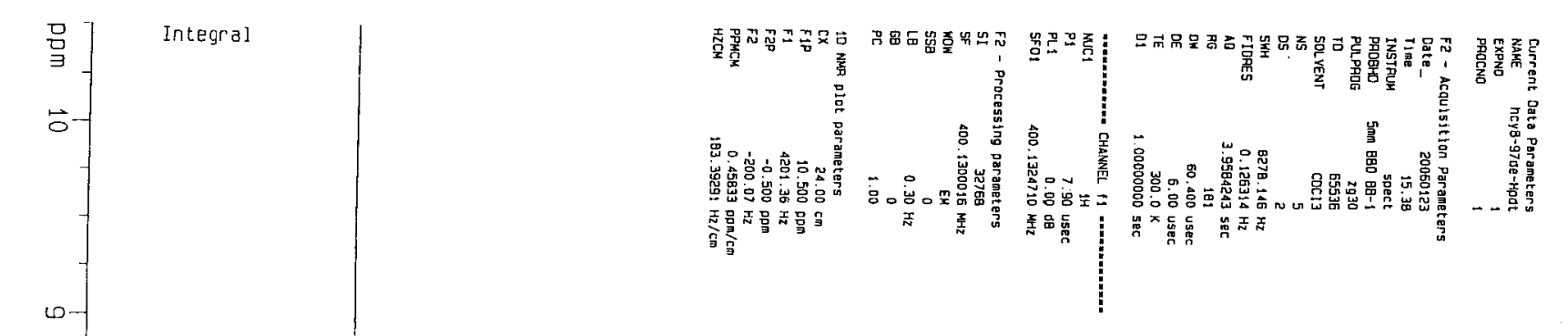

$I$
0
0
0
07
01
0
0
0
0
0
0
$0+$
0
0
5
5
9

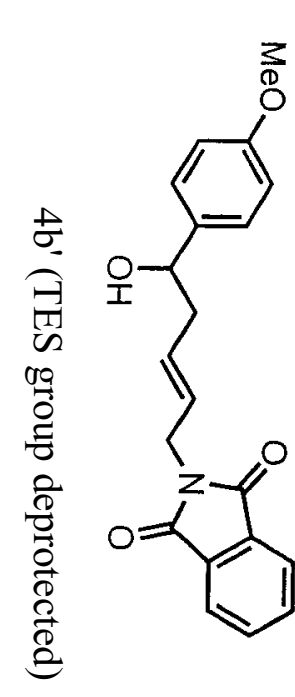




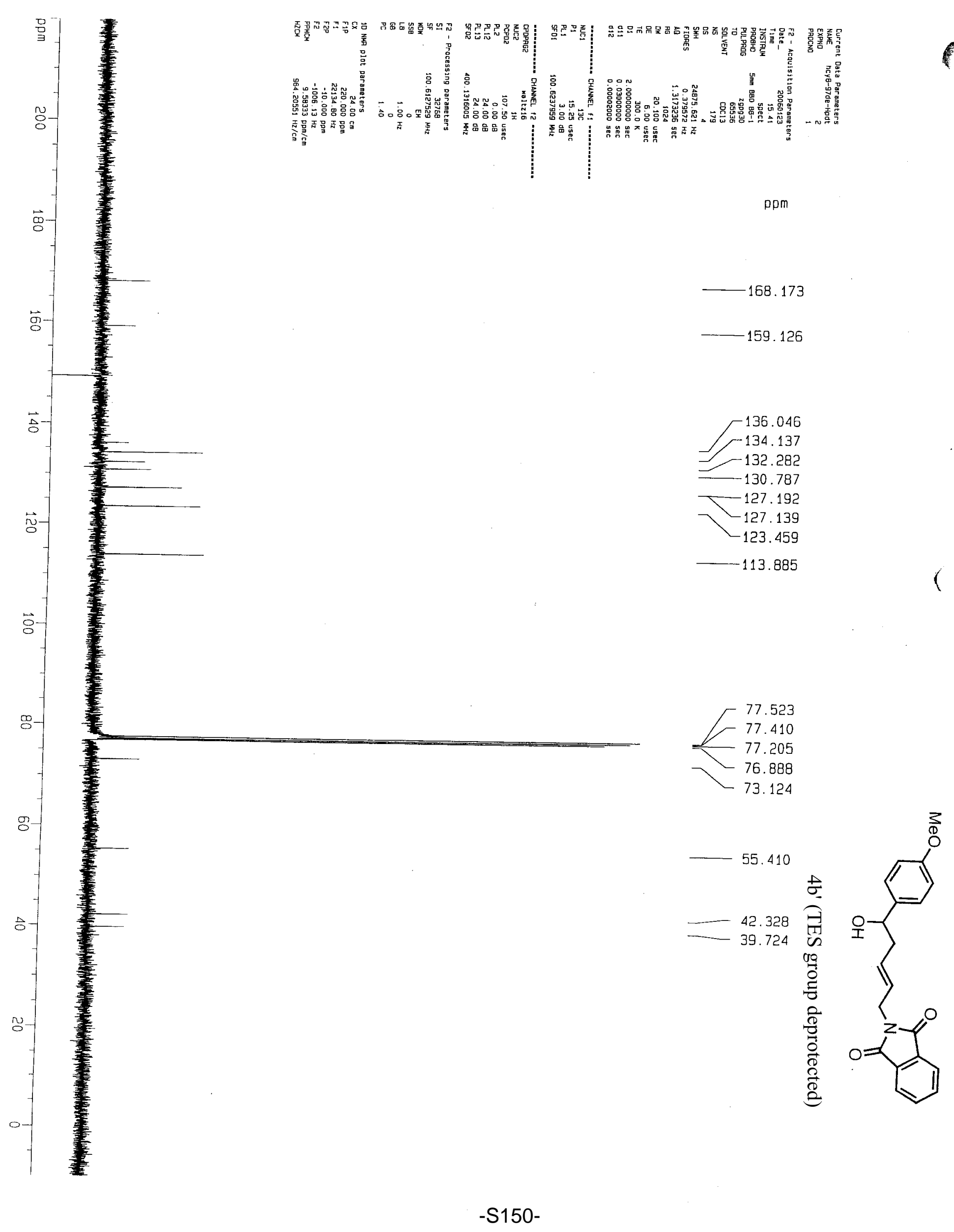




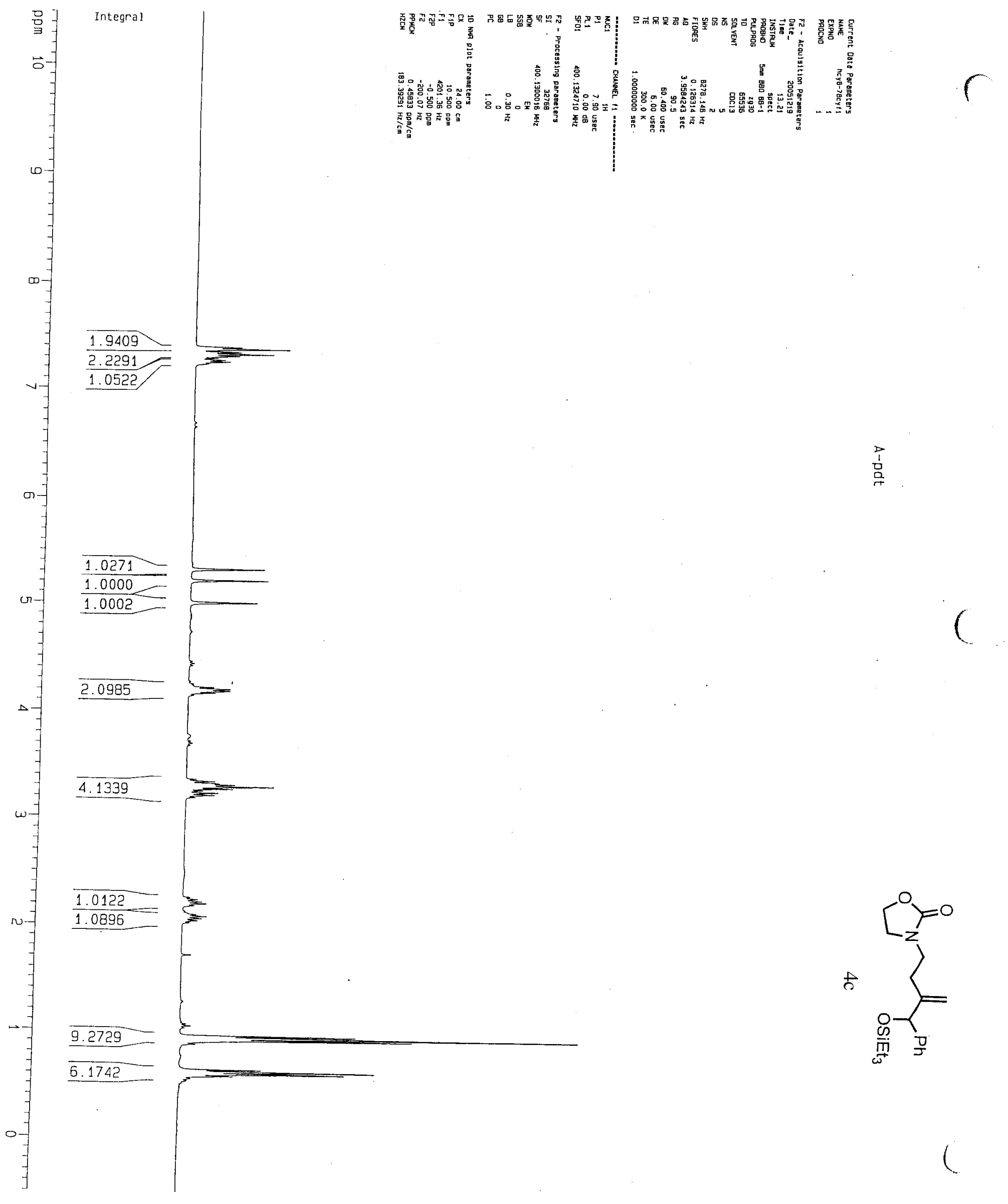




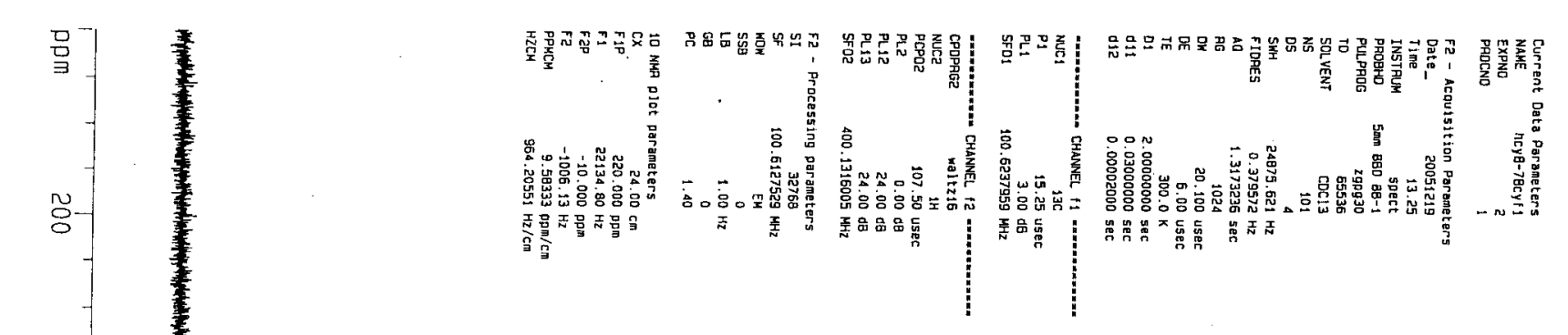

ppm
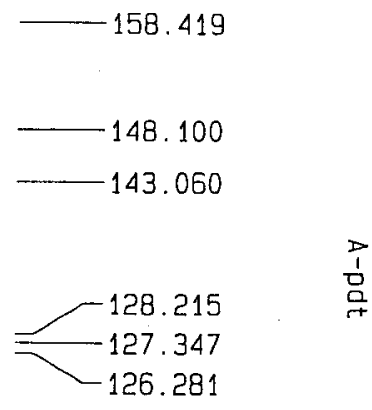

$-111.991$
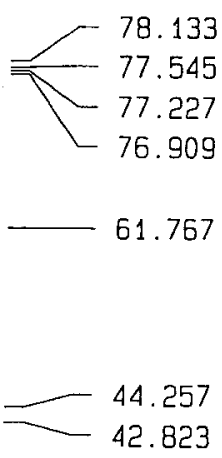

27.929

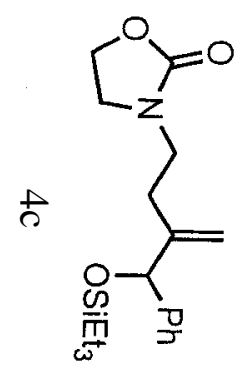

6.999

$=\quad 4.942$ 


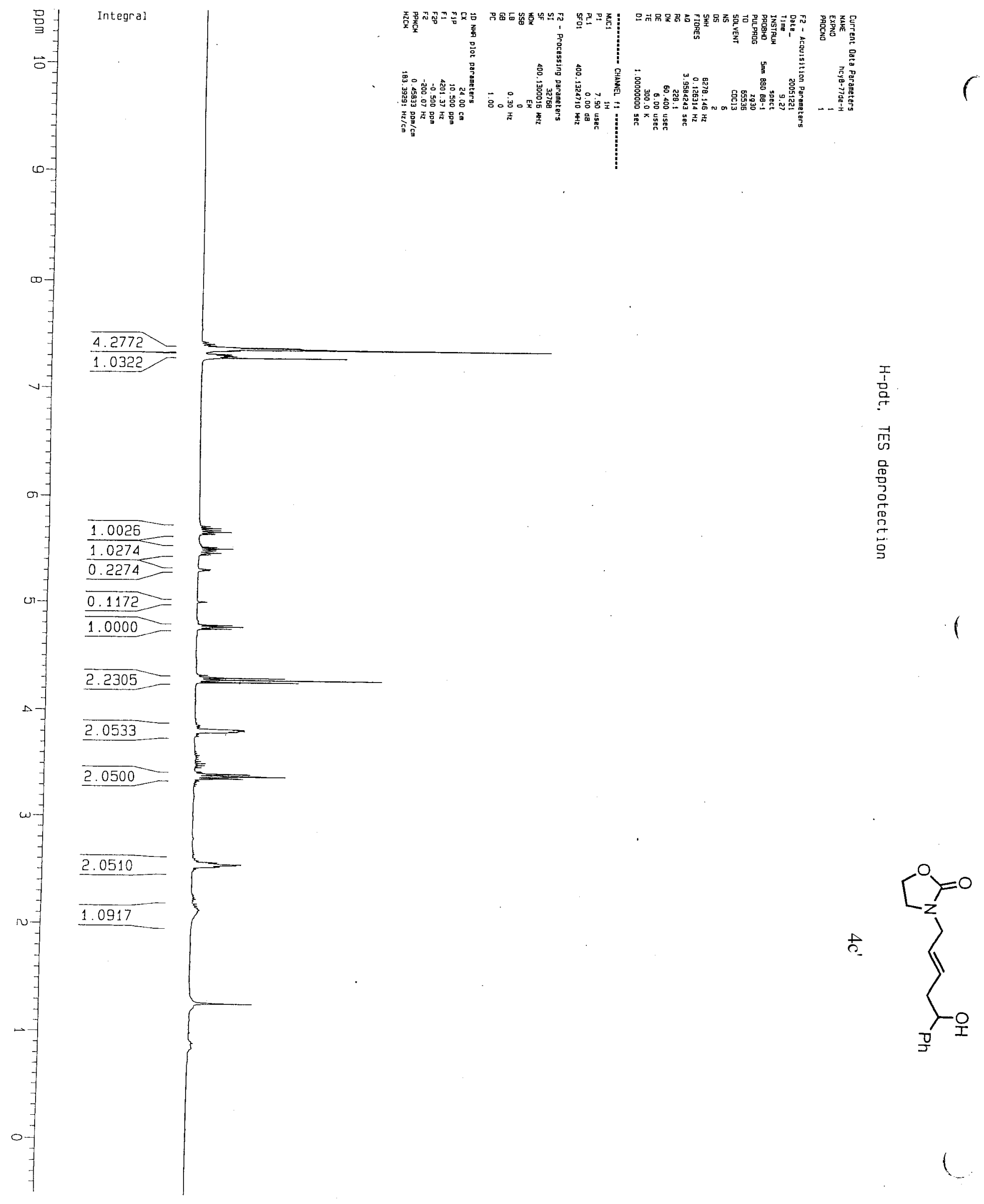




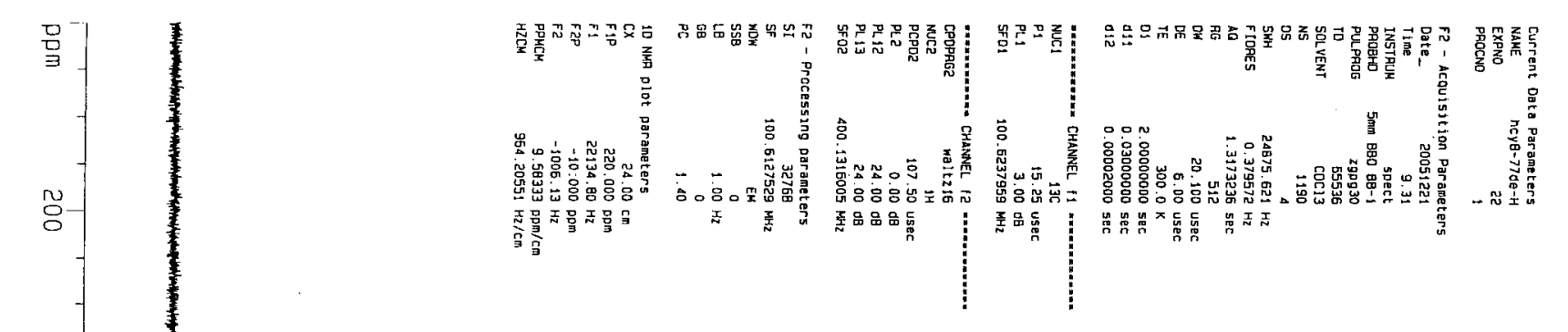

ppm

$$
\overrightarrow{\mathrm{g}}
$$

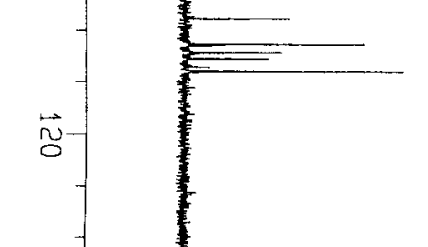

$\vec{\circ}$

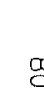

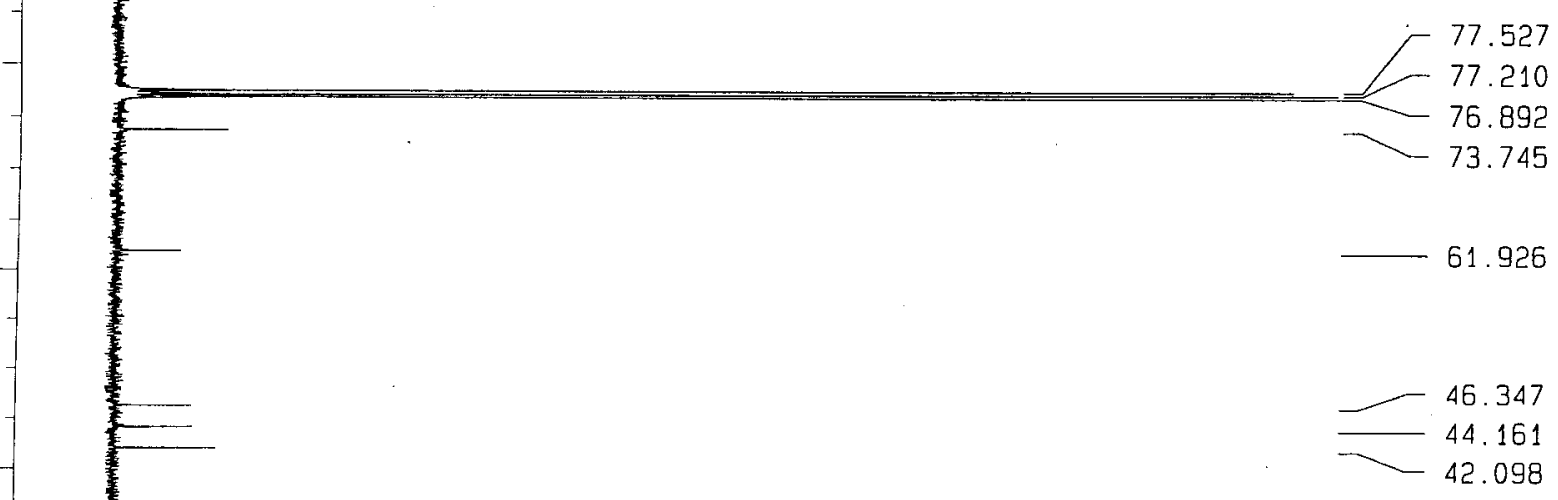


to $\$$ uproudg te 8 -han

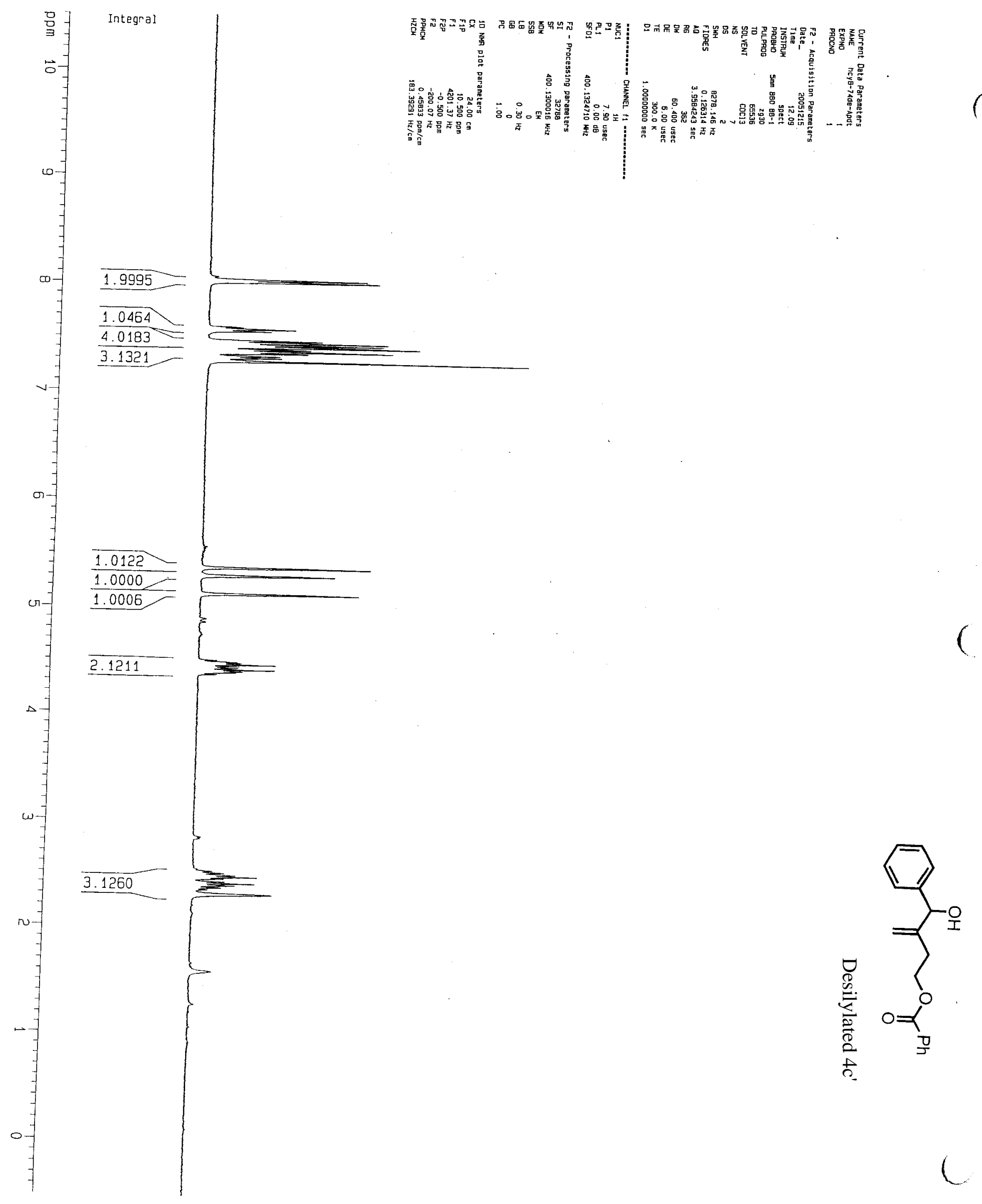



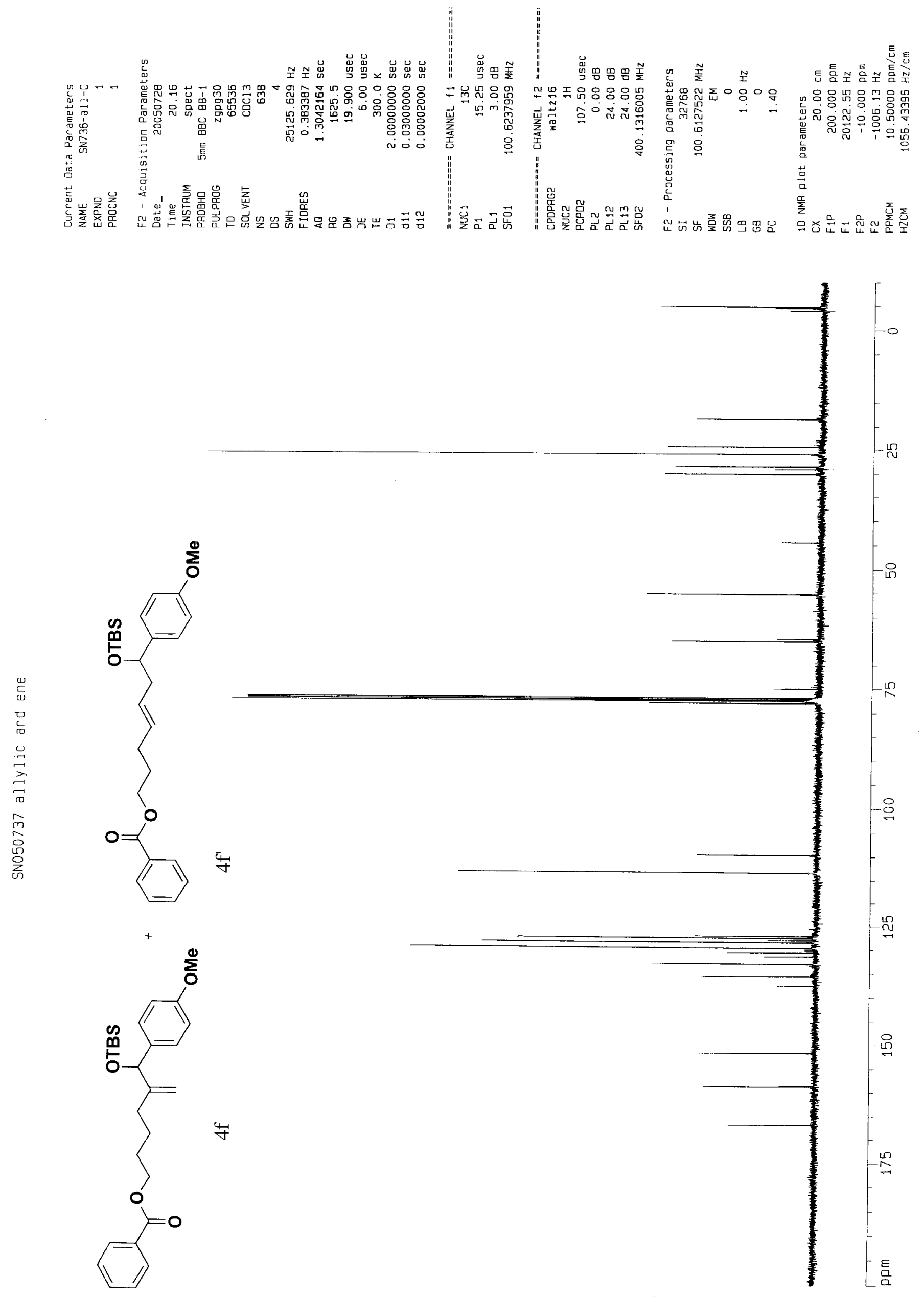

1 


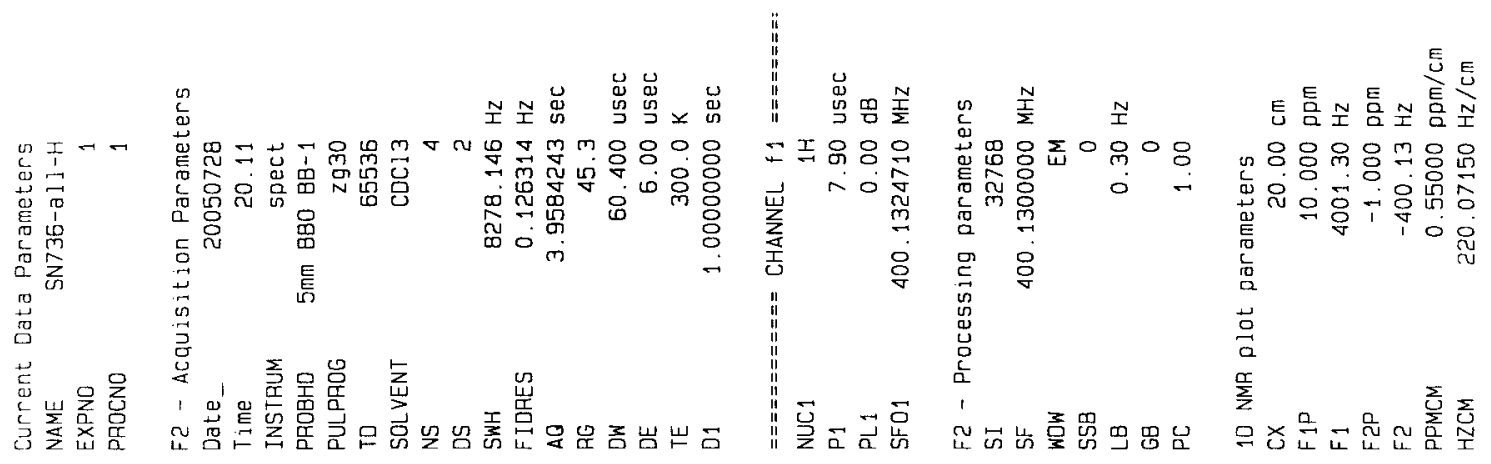

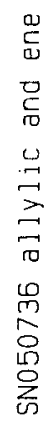

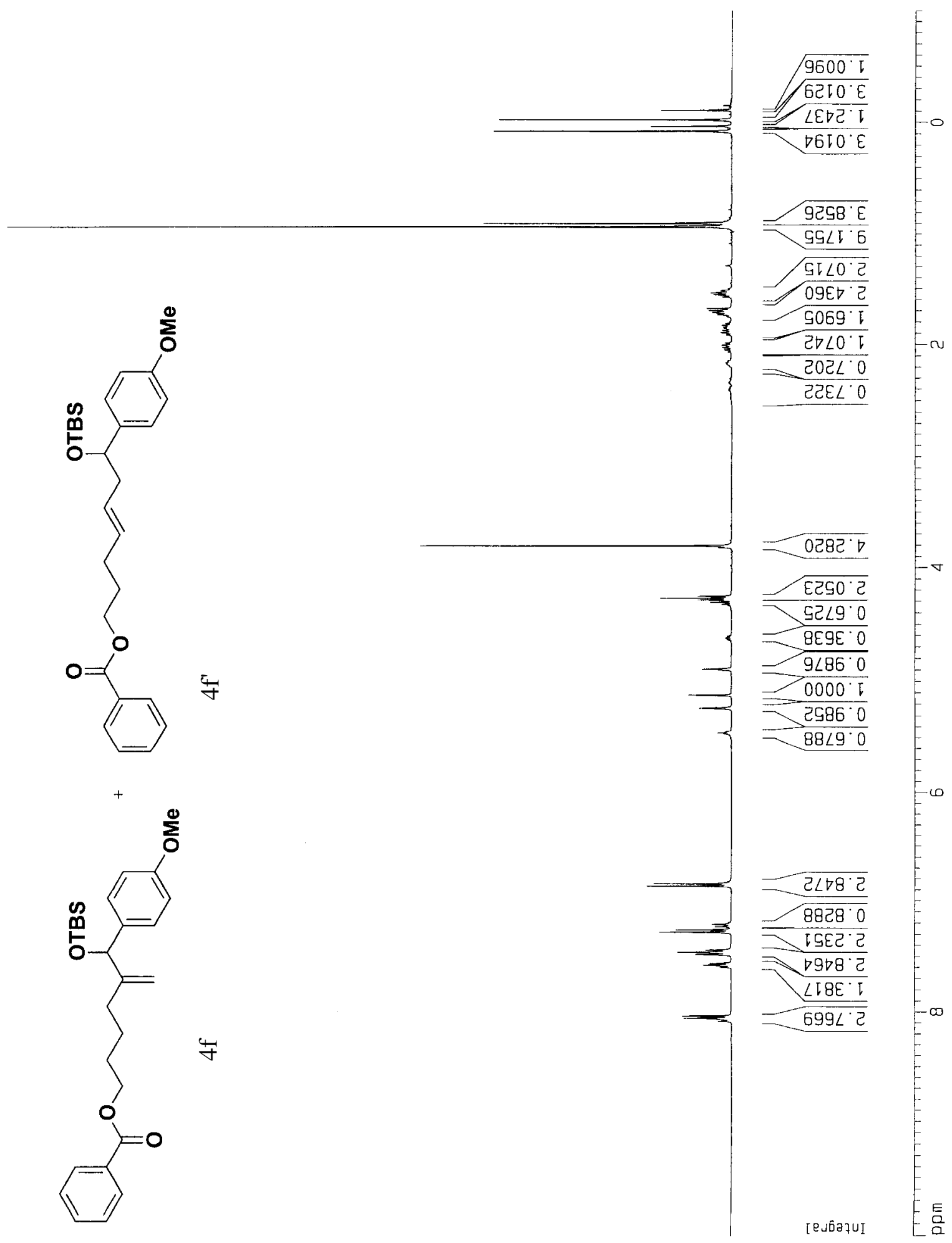




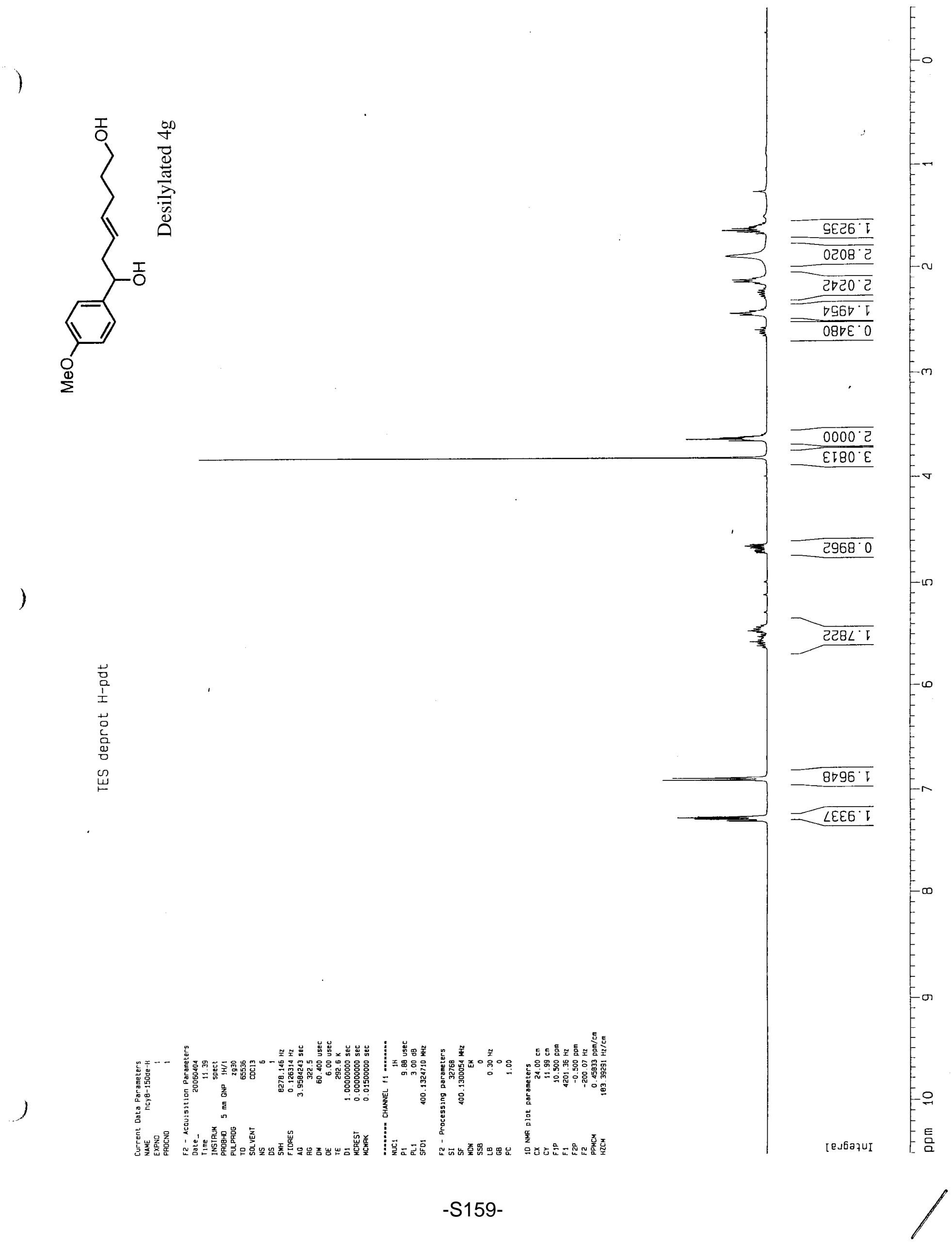




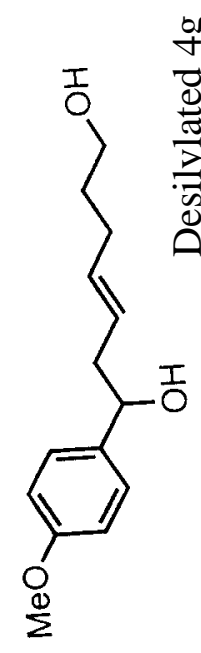

$E 99^{\circ} \varepsilon 2-$
ODG $62-$

$590^{\circ}$ टE

8टE'टE

$\triangle ट E^{\prime} \angle E$

$608 \cdot 2 b$

$680 \cdot 99-$

$\angle 96.19$

521.29

$E L \varepsilon^{\prime} E L$

$\nabla \angle 9^{\circ} E L \longrightarrow$

S06. $9 L$

टटट ' $\angle L$

ODS $L L$

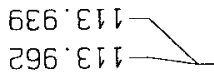

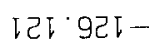

$069^{\circ} 951$

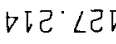

$9 \varepsilon 己{ }^{\circ} \angle \mathcal{I}$

68७ टहा

$92{ }^{\circ} \nabla E I-$

80t.9E -

$6 \triangleright g^{\prime} 9 \varepsilon$ I

805695

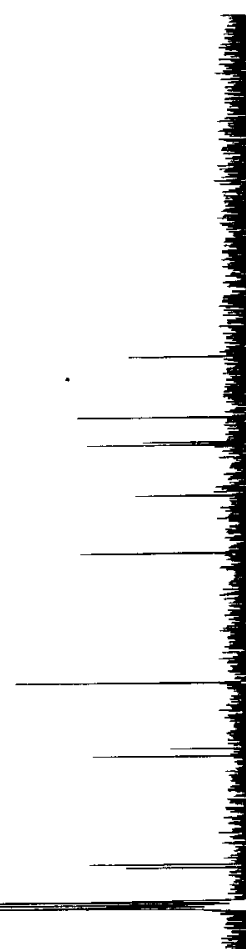




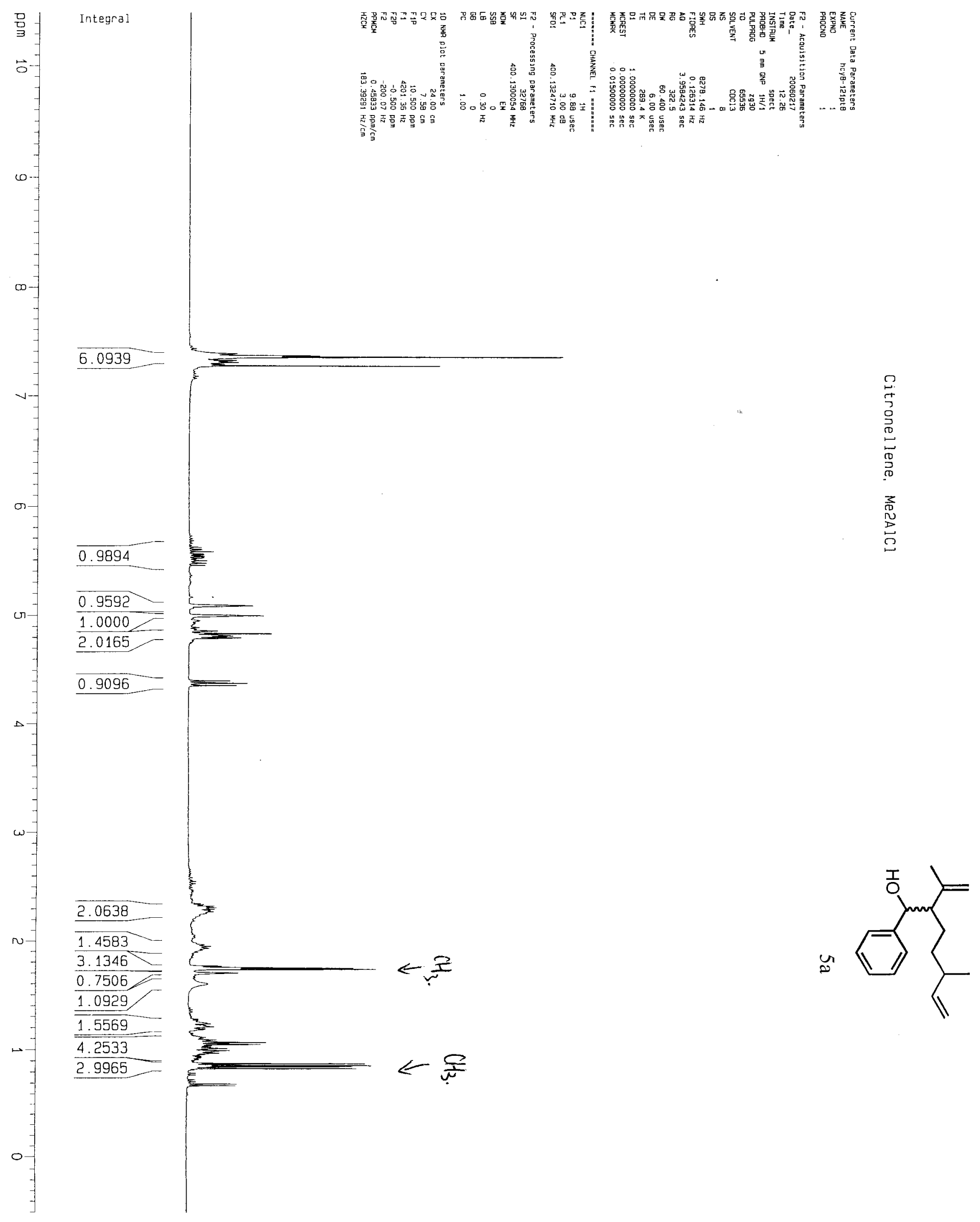



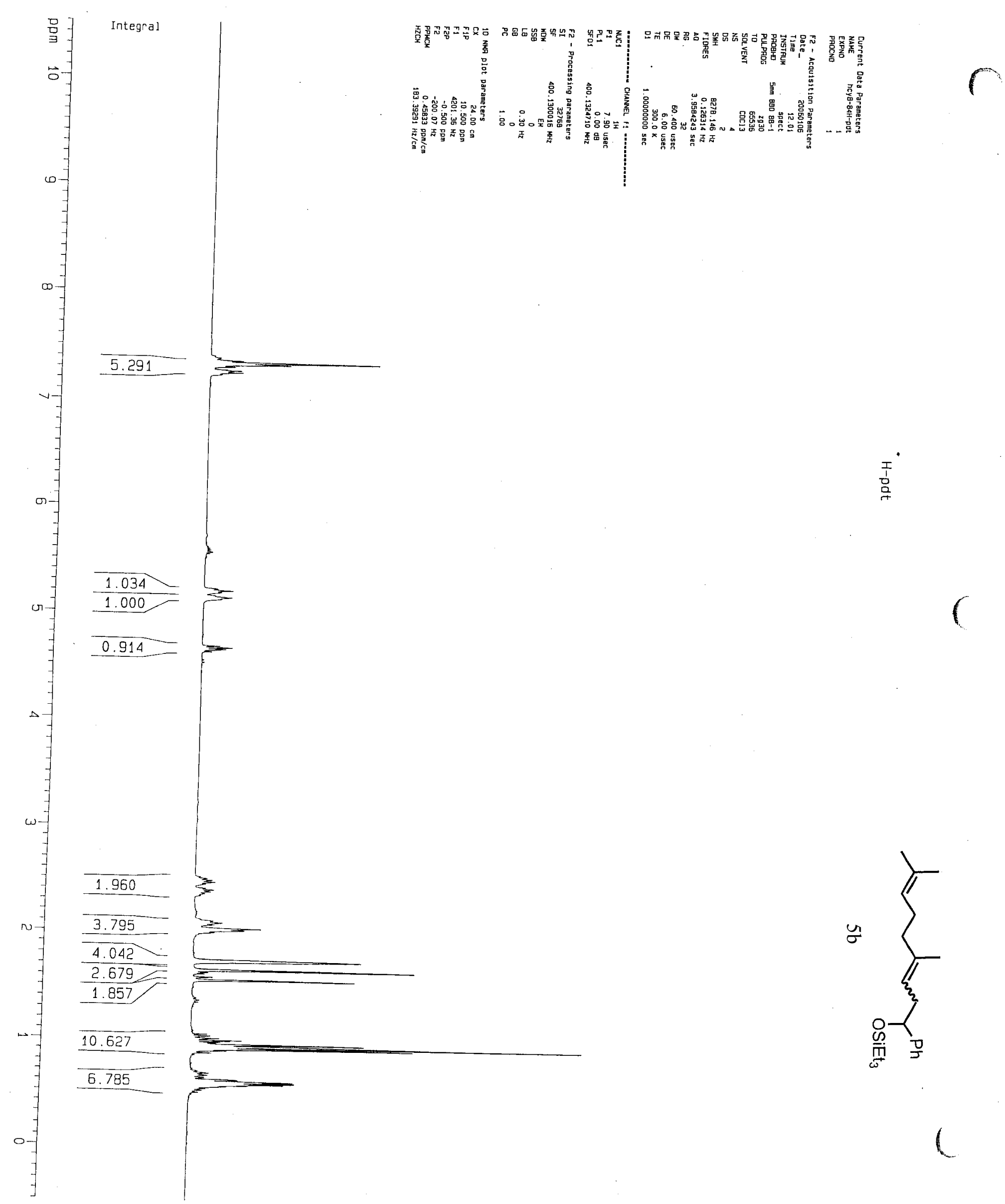

w
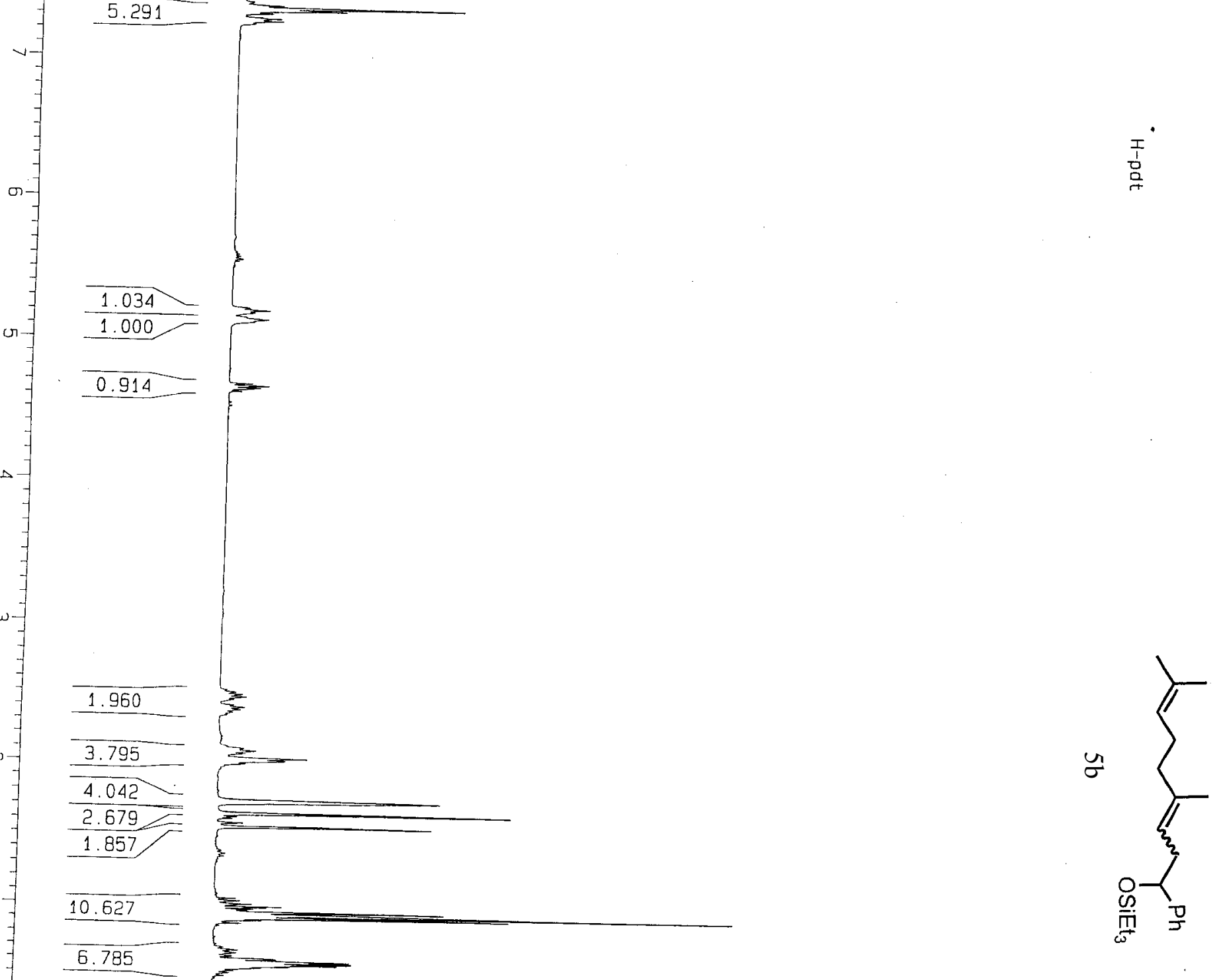


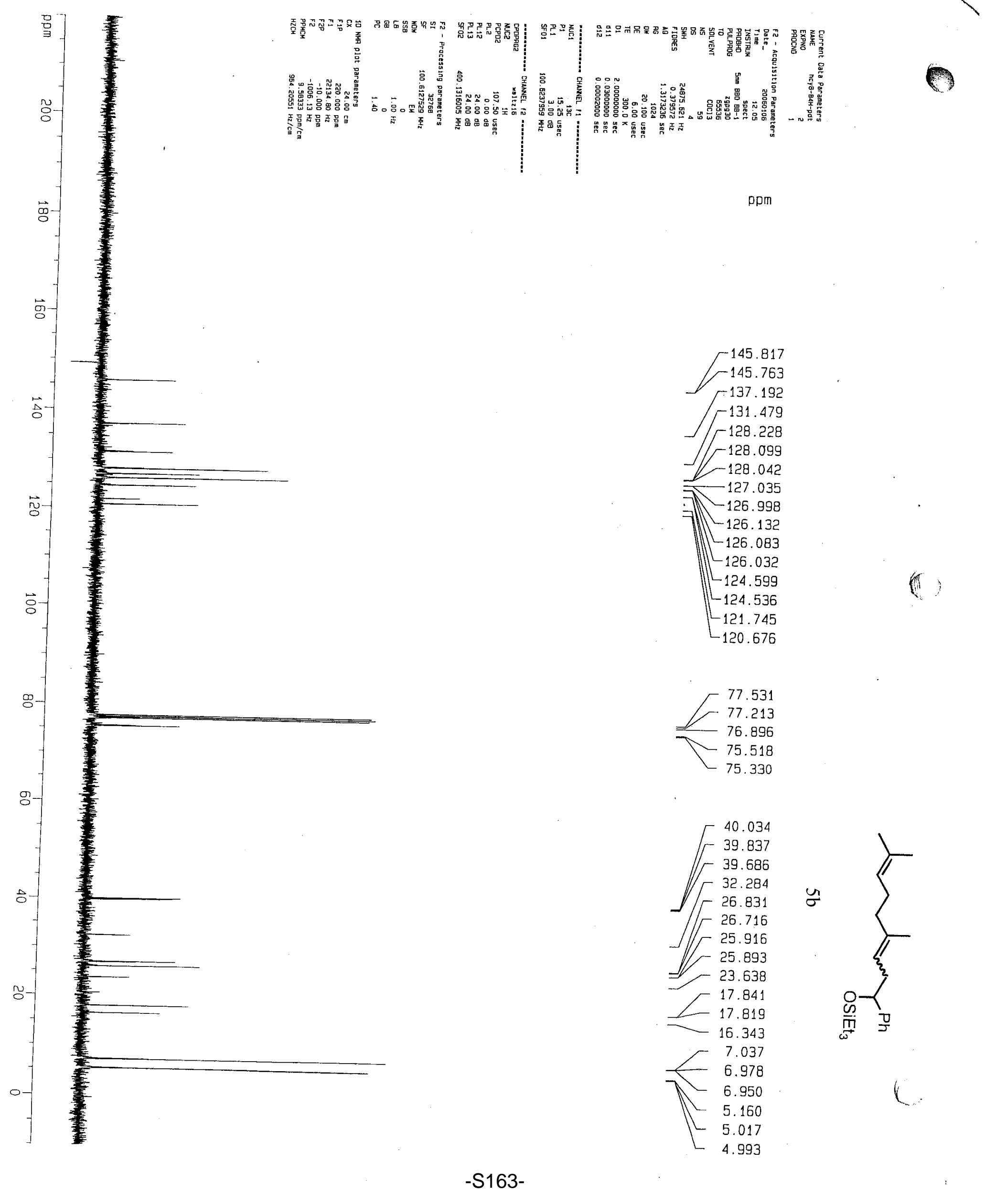



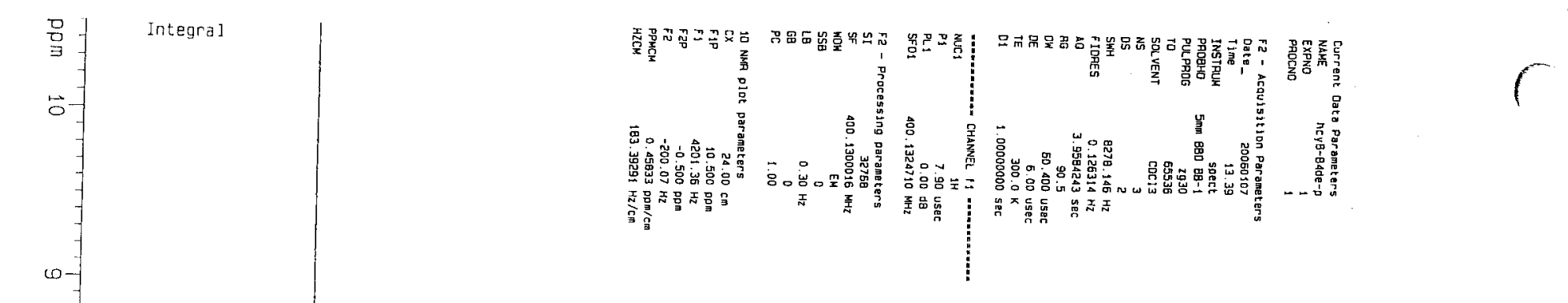

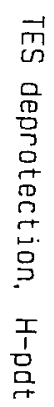

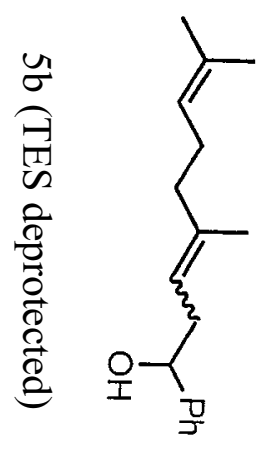

